\title{
Decoherence in \\ Infinite Quantum Systems
}

von

Mario Hellmich

Dissertation

zur Erlangung des Doktorgrades

der Fakultät für Physik der

Universität Bielefeld

Januar 2009 

1. Gutachter: Prof. Dr. Philippe Blanchard (Bielefeld)

2. Gutachter: Prof. Dr. Robert Olkiewicz (Breslau)

3. Gutachter: Prof. Dr. Gianfausto Dell'Antonio (Rom)

Tag der Disputation: 30. Januar 2009 


\section{Acknowledgments}

First and foremost I would like to express my heartfelt thanks to Prof. Dr. Philippe Blanchard for supervising this thesis. Through his enthusiasm for the subject and through many spirited discussions (not only about physics or mathematics) he had a great influence on this work, and on many occasions he has put me on the right track. His constant encouragement and support in scientific as well as organizatorial and fundraising matters have been a great help to me without which this thesis would not have come to completion.

I would like to thank Prof. Dr. Robert Olkiewicz for his fruitful collaboration and for sharing many of his ideas with me. It was always very inspiring and rewarding to discuss with him. His hospitality in Wrocław and his immediate willingness to write a report on this thesis are greatly appreciated.

Prof. Dr. Dell'Antonio provided the third report on this thesis, I would like to thank him for his interest and his efforts.

I am grateful to the International Graduate School "GRK 881" Bielefeld-Paris for financial support. Moreover, thanks are due to Evangelisches Studienwerk Villigst e. V. for funding a large part of this work and for the many experiences and opportunities beyond the financial support.

I would like to express my sincere thanks to our secretary Hannelore Litschewsky for the many favors she has done to me during the last few years and for always finding time for a chat.

Many people have influenced me, and hence this work, during its making, and I would like to thank them for what they have done for me. Special mention deserve my parents, without their support and encouragement I would have been lost on many occasions. Last, but not least, I am grateful to Hanna-Lena for cheering me up in difficult moments. 


\section{Contents}

$\begin{array}{ll}\text { Introduction } & 1\end{array}$

1. Quantum Probability and Open Systems 5

1.1. Mathematical Description of Physical Systems . . . . . . 5

1.1.1. States and Observables . . . . . . . . . . 5

1.1.2. C*-Algebraic Description of Physical Systems . . . . 7

1.1.3. C*-Algebras and von Neumann Algebras . . . . . . . 10

1.2. Quantum Probability and Physical Systems . . . . . . . . . 14

1.2.1. Algebraic Classical Probability . . . . . . . . . . 15

1.2.2. The Quantum Generalization . . . . . . . . . . . 17

1.2.3. Classical Physical Systems . . . . . . . . . . . . 18

1.2.4. Quantum Systems . . . . . . . . . . . . . 20

1.2.5. The Representation Problem . . . . . . . . . . . 22

1.2.6. Infinite Systems . . . . . . . . . . . . . 27

1.2.7. Differences Between Classical and Quantum Systems 29

1.3. Open Systems and Dynamics . . . . . . . . . . . . . 33

1.3.1. Dynamics of Subsystems and Conditional Expectations . . . . . . . . . . . . . 33

1.3.2. Reduced Dynamics and Dynamical Semigroups . . . 36

1.4. Fixed Points and Invariant States . . . . . . . . . . . . . . 39

1.4.1. Fixed Points . . . . . . . . . . . . . . 39

1.4.2. Almost Periodicity . . . . . . . . . . . . . . . . 40

1.4.3. Amenability and Mean Ergodicity . . . . . . . . . 44

1.4.4. Invariant and Subinvariant States . . . . . . . . . . 49

2. Decoherence: Technical Tools 51

2.1. Mathematical Preliminaries . . . . . . . . . . . 51

2.1.1. Tomita-Takesaki Theory . . . . . . . . . . . 51

2.1.2. The KMS Condition . . . . . . . . . . . . . . 53

2.2. Asymptotic Splittings . . . . . . . . . . . . . . . 54

2.2.1. Isometric-Sweeping Splitting . . . . . . . . . . 54

2.2.2. Jacobs-de Leeuw-Glicksberg Splitting . . . . . . . 59

2.2.3. An Example . . . . . . . . . . . . . . . . 67 
2.3. Detailed Balance . . . . . . . . . . . . . . . . . . 69

2.3.1. Definition and Properties . . . . . . . . . . . 69

2.3.2. Equivalent Characterization of Detailed Balance . . 74

2.3.3. Sufficient Conditions for Detailed Balance . . . . . . 80

2.3.4. Detailed Balance and Approach to Equilibrium . . . 82

3. Decoherence: Physical Interpretation 85

3.1. Decoherence in the Algebraic Formulation . . . . . . . . . 85

3.1.1. Introduction . . . . . . . . . . . . . . 85

3.1.2. The Notion Decoherence in Algebraic Formulation . 88

3.2. Some General Theorems on Decoherence . . . . . . . . . . . 90

3.2.1. Sufficient Conditions . . . . . . . . . . . . . 90

3.2.2. Structure of the Algebra of Effective Observables . . 93

3.2.3. Scenarios of Decoherence . . . . . . . . . . . . 97

3.2.4. Mesurements . . . . . . . . . . . . . . . . 99

4. Irreversible Evolutions on the CCR 103

4.1. Quasifree Maps . . . . . . . . . . . . . . . . . . . . . 104

4.2. Perturbed Convolution Semigroups . . . . . . . . . . 105

4.2.1. Definition . . . . . . . . . . . . . . 105

4.2.2. Construction of Perturbed Convolution Semigroups . 106

4.2.3. General Representation of a Perturbed Convolution Semigroup . . . . . . . . . . . . . . . 108

4.3. Dynamical Semigroups on $\mathfrak{A}(\mathrm{S}, \sigma) \ldots \ldots \ldots$

4.3.1. Symplectic Groups . . . . . . . . . . . . . . . 110

4.3.2. Nonsymplectic Semigroups . . . . . . . . . . . . 111

4.4. Extension to Representations of the CCR Algebra . . . . . 119

4.4.1. The Extension Theorem . . . . . . . . . . . . . . . 119

4.4.2. A Generalization of the Extension Theorem . . . . . 122

4.5. Generators . . . . . . . . . . . . . . . . 124

4.5.1. The Gaussian Case . . . . . . . . . . . . . . . 125

4.5.2. The Poisson Case . . . . . . . . . . . . . . . . . 130

4.5.3. The Dirac Case . . . . . . . . . . . . . . . . . 131

4.6. Normal Invariant States . . . . . . . . . . . . . . . . . . 132

4.7. Detailed Balance for Quasifree Semigroups . . . . . . . . . 135

4.7.1. General Condition . . . . . . . . . . . . . . 135

4.7.2. Example . . . . . . . . . . . . . . . 136

5. Conclusion and Open Problems 139

5.1. Conclusion . . . . . . . . . . . . . . . . . . . 139

5.2. Open Problems . . . . . . . . . . . . . . . . . . 141 
A. The CCR Algebra

A.1. Definition and Properties . . . . . . . . . . . . . 145

A.1.1. Definition . . . . . . . . . . . . . . 145

A.1.2. Properties . . . . . . . . . . . . . . . 147

A.1.3. Generating Functionals . . . . . . . . . . . 150

A.1.4. Automorphisms . . . . . . . . . . . . . . . 151

A.2. Regular and Quasifree Representations . . . . . . . . . 152

A.2.1. Regular Representations . . . . . . . . . . . . 153

A.2.2. Quasifree States and Representations . . . . . . . . 158

B. Extension of Semigroups 161

B.1. Extension of Semigroups on Banach Spaces . . . . . . . 162

B.2. Extension of Semigroups on von Neumann Algebras . . . 166

C. Nuclear Spaces and Maps 169

C.1. Nuclear Maps . . . . . . . . . . . . . . . . . . . . . . . . . . 169

C.2. Nuclear Spaces . . . . . . . . . . . . . . . . . . . 172

D. Cylindrical Measures $\quad 177$

D.1. Cylindrical Measures and Functions . . . . . . . . . . 177

D.1.1. Cylinder Sets . . . . . . . . . . . . . . 177

D.1.2. Cylindrical Measures . . . . . . . . . . . . . . . 179

D.2. The Generalized Minlos-Sazonov Theorem . . . . . . . . . 181

$\begin{array}{ll}\text { Bibliography } & 183\end{array}$

$\begin{array}{ll}\text { Summary } & 191\end{array}$ 



\section{Introduction}

The theory of environmental decoherence starts from the question why the objects surrounding us obey the laws of classical physics, despite the fact that our most fundamental physical theory - quantum theory - results in contradictions to experimental and everyday experience when directly applied to these objects. This is an embarrassing situation since from its inception in the 1920's until today quantum theory has seen a remarkable success and an ever increasing range of applicability. Thus the question how to reconcile quantum theory with classical physics is a fundamental one, and efforts to find answers to it persisted throughout its history. Although there is still no general consensus how an answer can be achieved, environmental decoherence today is the most promising one and the one most widely discussed.

The answer offered by the program of environmental decoherence to the question posed above is that quantum theory is universally valid, in particular also in the macroscopic domain, but that one has to take into account the fact that macroscopic objects are strongly interacting with their environment, and that precisely this interaction is the origin of classicality in the physical world. Thus classicality is a dynamically emergent phenomenon due to the interaction of quantum systems with other quantum systems surrounding them.

In the past twenty years decoherence has been the subject of intensive theoretical and experimental research and since then has generated an enormous amount of literature. A search in the Science Citation Index for "decoherence" yields well over 5000 hits. Despite this massive progress there is still a lot of discussion about the meaning, prospects and possibilities of decoherence going on. In order to clarify the status of decoherence and to provide a rigorous definition, $\mathrm{Ph}$. Blanchard and $\mathrm{R}$. Olkiewicz suggested a notion of decoherence formulated in the algebraic framework of quantum physics in [46], drawing on earlier work of R. Olkiewicz [105]. The algebraic framework is an alternative mathematical formulation of quantum physics besides the traditional textbook formulation, which is contained in the algebraic framework as a special case. However, it is more powerful since it can also accommodate classical systems, provides an elegant formulation of superselection rules and can accommodate systems 
with infinitely many degrees of freedom rigorously, which is important in order to discuss systems in the thermodynamical limit and in quantum field theory. Thus it is particularly well suited for the discussion of the dynamical emergence of classical properties. Recently the algebraic formulation becomes increasingly popular in the discussion of foundational and philosophical problems of quantum physics, see e.g. [90], and the work of Ph. Blanchard and R. Olkiewicz on decoherence was appraised by N. P. Landsman who declared that it "should provide important motivation for specialists in the foundations of quantum theory to learn the theory of operator algebras" [91].

To advertise the algebraic framework, especially for our purpose, we start in Chapter 1 by explaining briefly its key features; in particular, we emphasize that the formalism of quantum theory may fruitfully be viewed as a generalization of classical Kolmogorovian probability theory. In fact, as R.F. Streater contends, one should view "quantum theory is a generalisation of probability, rather than a modification of the laws of mechanics" [123]. In Chapter 1 we also introduce the necessary mathematical tools to describe the dynamics of open systems, in particular quantum dynamical semigroups, which are fundamental for decoherence, as well as a number of useful mathematical tools.

In Chapter 2 we develop the mathematical machinery which will allow us to prove that a given quantum dynamical semigroup displays the decoherence effect. Besides giving a new proof for a special case of a theorem of Ługiewicz and Olkiewicz [94] we will also discuss the Jacobs-de LeeuwGlicksberg splitting for quantum dynamical semigroups as well as a notion of detailed balance which will lead to a sufficient condition for a quantum dynamical semigroup to display decoherence in the sense of Blanchard and Olkiewicz.

In Chapter 3 we will introduce the notion of decoherence due to $\mathrm{Ph}$. Blanchard and R. Olkiewicz and, following [46], discuss its consequences. Physically its main virtue lies in a classification of the possible scenarios of decoherence and thus provides an answer to the question about the possibilities of decoherence. Here one clearly sees that decoherence need not always lead to classical properties but can also result in new quantum properties of a quantum system. Moreover, it becomes apparent that there is no intrinsic limit of decoherence to macroscopic systems. From our work of Chapter 2 we will be able to give a number of sufficient conditions for a quantum dynamical semigroup to display decoherence; the detailed balance condition is a new aspect here. We will not go into interpretational issues related to decoherence, for such aspects see the literature $[137,117]$.

Up to here our work was geared toward general aspects, having in- 
troduced a broad framework for the discussion of decoherence as well as general conditions for a quantum dynamical semigroup to display decoherence. Due to the use of the algebraic framework our results are sufficiently general to treat also infinite systems. On the other hand, if we want to explore decoherence in infinite quantum systems, it is important to have a number of physical examples of infinite open quantum systems available. A first step toward this goal is attempted in Chapter 4. There we construct of a class of quantum dynamical semigroups on von Neumann algebras corresponding to representations of the algebra of the canonical commutation relations, which describe (not necessarily finite) Bose systems. In particular, our results will enable to construct quantum dynamical semigroups describing infinite open systems. Since there are not many quantum dynamical semigroups on type III von Neumann algebras known in the literature, this is an important first step toward physical models of decoherence in infinite systems. Since our construction is obtained by extending semigroups we develop some useful technical results in this direction in Appendix B. We investigate the quantum dynamical semigroups thus obtained in Chapter 4, derive explicit expressions for their generators and also discuss the existence of faithful normal invariant states.

The final Chapter 5 contains a brief summary of this work and closes with some open problems and proposals for what might been worth investigating. 


\section{Chapter 1.}

\section{Quantum Probability and Open Systems}

This chapter starts by motivating the algebraic description of physical systems, starting from operational principles. We explain how classical and quantum systems are described within the algebraic formulation and introduce the basic idea of quantum probability theory. In particular, we stress that the description of infinite systems needs a generalization of the usual textbook quantum mechanics for systems with finitely many degrees of freedom. Then we introduce the mathematical description of open systems and their irreversible dynamics. The last section gives a number of technical results concerning invariant states and fixed points for irreversible evolutions.

\subsection{Mathematical Description of Physical Systems}

\subsubsection{States and Observables}

In this section we introduce the two notions of state and observable, mainly following the setup given in [2]. In every physical theory they have direct operational significance, here we shall try to give a general mathematical model which captures their essential features. It is believed that every physical theory can be described in these abstract terms and we argue that there is moreover a common mathematical framework in which states and observables can be discussed. This will allow us to treat both classical and quantum systems in the same mathematical framework, which is an important aspect for our purpose since we want to study situations in which quantum systems develop classical behavior, so a common mathematical framework is certainly useful. Moreover, we believe that foundational issues of quantum physics are best discussed in this framework. 
Consider a typical laboratory experiment involving some physical system. In every such experiment we can identify two subsequent phases: preparation and measurement. At first we subject the system to some preparation procedure which ensures that upon repetition of the experiment we can be sure that we set out from the same initial condition. After preparation we allow the system to evolve freely until we perform a measurement on it, that is we cause an interaction of the system and another system, designed specifically for this purpose - the measuring instrument. This will change the state of the instrument (and, in general, of the system as well) in some way which may be recorded, for example by moving the pointer of a dial. The reading of the dial is the result of the measurement. Now we find that a measurement performed on two identical systems which have been subjected to identical preparation procedures can lead to different results, and conversely, that two identical systems which have been prepared differently can yield identical results. Hence we must take this indeterminacy into account in a theoretical description of this situation. We will assume that a specific preparation and a specific measurement do not determine a unique outcome with certainty but only a probability distribution on the set of all possible outcomes of the measurement. The probability distribution may then be tested against experiment by performing the measurement a large number of times on identically prepared systems and comparing the probabilities of our theoretical description with relative frequencies of the outcomes; in this way we decide if a particular theoretical description is tenable or not.

To build up a mathematical theory describing this situation we must associate mathematical objects to the preparation and measurement process such that they are able to predict a probability distribution for each pair of a preparation and a measurement. A preparation procedure will be denoted by $\varphi$, it can be thought of as a concrete recipe of how to set up the system; a measurement effected by using a specific instrument will be denoted by $A$; as for preparation procedures $A$ can be thought of as the design drawing of the instrument. The probability distribution predicted by the theory, say on the Borel sets of the real numbers $\mathbb{R}$, will be denoted by $P[\varphi, A ; \cdot]$, where $P[\varphi, A ; E]$ is the probability that we find the event (outcome) $E$ in the measurement, i, e. that the dial shows a reading lying in the Borel set $E \subseteq \mathbb{R}$. To set up a mathematical theory we first have to evade a slight difficulty. It is of course possible to have two different preparation procedures $\varphi_{1}$ and $\varphi_{2}$ such that the probability distributions $P\left[\varphi_{1}, A ; \cdot\right]$ and $P\left[\varphi_{2}, A ; \cdot\right]$ are identical for any instrument $A$ 
we have at our disposal ${ }^{1}$, if they only differ in irrelevant details not seen by the instruments. Then we define $\varphi_{1}$ and $\varphi_{2}$ as equivalent, in symbols $\varphi_{1} \sim \varphi_{2}$. It is easy to see that $\sim$ defines an equivalence relation on the set of preparations. Clearly, two preparations $\varphi_{1}$ and $\varphi_{2}$ with $\varphi_{1} \sim \varphi_{2}$ should be identified in a theoretical description, therefore we pass to the equivalence classes of preparation procedures. Such an equivalence class is called a state, the set of all states will be denoted by $\Sigma$. Similarly, if for two instruments $A_{1}$ and $A_{2}$ the probability distributions $P\left[\varphi, A_{1} ; \cdot\right]$ and $P\left[\varphi, A_{2} ; \cdot\right]$ agree for all states $\varphi \in \Sigma$ we call the instruments equivalent, in symbols $A_{1} \sim A_{2}$, and the equivalence classes of this equivalence relation are called observables, the set of all observables will be denoted by $\mathfrak{O}$. For a pair $A \in \mathfrak{O}$ and $\varphi \in \Sigma$ we write $\varphi(A)$ for the expectation value of the probability distribution $P[\varphi, A ; \cdot]$.

\subsection{2. $C^{*}$-Algebraic Description of Physical Systems}

In the previous section we have established the notion of a set of states $\Sigma$ and observables $\mathfrak{O}$, and introduced a pairing $\langle A, \varphi\rangle=\varphi(A) \in \mathbb{R}$ for $\varphi \in \Sigma$ and $A \in \mathfrak{O}$, the expectation value. Using the operational significance of $\Sigma$ and $\mathfrak{O}$ and the pairing $\varphi(A)$ it is possible to argue in favor of a general mathematical model for $\Sigma$ and $\mathfrak{O}$. Such an argument is not meant to be a derivation of a certain mathematical framework, which is impossible in a strict logical sense, it is rather to say that there is a mathematical framework which covers all known physical applications and admits a sufficiently rich structure to faciliate rigorous developments. This mathematical framework we are about to introduce is generally known as the algebraic framework of (quantum) physics. It was pioneered by Segal [119] and developed by Haag and Kastler [76], see [10] for a historical account and bibliography as well as [28] for a short and elementary account.

We will not give complete arguments here but instead refer to the literature cited above, we shall only try to convey some key points. First we observe that if $\varphi(A)=\varphi(B)$ for any $\varphi \in \Sigma$ the observables $A, B \in \mathfrak{O}$ are not distinguishable by any measurement, hence, by what was said above, they are identified: $A=B$. Similarly, if $\varphi_{1}(A)=\varphi_{2}(A)$ for any $A \in \mathfrak{O}$ then $\varphi_{1}=\varphi_{2}$. This translates into the mathematical statement that the states separate the observables and vice versa. Now we establish some algebraic structure on $\mathfrak{O}$. Let $A \in \mathfrak{O}$ and $\lambda \in \mathbb{R}$. Since $A$ characterizes

\footnotetext{
${ }^{1}$ By choosing how many instruments we include in our considerations we decide about the coarseness of our theoretical description. For example, if we think of a particle with spin, we can ignore its spin degree of freedom if we only include instruments that measure its spatial degrees of freedom and if there is no spin-orbit interaction.
} 
a concrete measuring apparatus, we can rescale it by $\lambda$ and thus obtain the observable $\lambda A \in \mathfrak{O}$. Then we should have $\varphi(\lambda A)=\lambda \varphi(A)$ for any $\varphi \in \Sigma$ by the interpretation of $\varphi(A)$ as an expectation value. Moreover, for $A \in \mathfrak{O}$ we can square the scale of the apparatus and thus obtain the observable $A^{2} \in \mathfrak{O}$, and more generally $A^{n}$ for any $n \in \mathbb{N}$. This permits us to calculate the $n$-th moments $\varphi\left(A^{n}\right)$ of the observable $A$ from which we can reconstruct the probability distribution (Hamburger moment problem, see below) of $A$ in state $\varphi$, hence it is sufficient to know the expectation values of all observables $\mathfrak{O}$ in any state in $\Sigma$ to get all the mathematical framework is able to predict.

In many situations we can find for two observables $A, B \in \mathfrak{O}$ (e.g. potential and kinetic energy) a third observable $C \in \mathfrak{O}$ such that $\varphi(C)=$ $\varphi(A)+\varphi(B)$ for any $\varphi \in \Sigma$. Then we write $C=A+B$. We shall assume that for any pair of observables $A, B$ there is a third, $C$, such that $C=A+B$ (if not $\mathfrak{O}$ has to be appropriately completed). With this structure $\mathfrak{O}$ becomes a real vector space and each $\varphi$ is a linear functional on $\mathfrak{O}$. Furthermore, we have a notion of positivity: If $A \in \mathfrak{O}$ is such that $\varphi(A) \geq 0$ for any $\varphi \in \Sigma$, i.e. a measurement of $A$ gives only positive results, then we call $A$ positive, in symbols $A \geq 0$. Since the square $A^{2}$ only gives positive results we stipulate that $A^{2} \geq 0$ for any $A \in \mathfrak{O}$. For the trivial observable $\mathbb{1} \in \mathfrak{O}$ which is constantly equal to 1 we require of course that $\varphi(\mathbb{1})=1$.

In a next step we introduce a norm on the vector space $\mathfrak{O}$. For any $A \in \mathfrak{O}$ we define

$$
\|A\|=\sup \{|\varphi(A)|: \varphi \in \Sigma\} .
$$

It can be checked that $\|\cdot\|$ indeed satisfies all properties required for a norm [28]. Moreover, it follows that $|\varphi(A)| \leq\|A\|$, i. e. $\varphi \in \Sigma$ is a continuous linear functional on the normed space $\mathfrak{O}$. From the definition of the norm it follows from $\varphi(\|A\| \mathbb{1} \pm A) \geq 0$ for all $\varphi \in \Sigma$ that

$$
\left\|A^{2}\right\|=\|A\|^{2}
$$

for any $A \in \mathfrak{O}$.

The fact that we were able to introduce the $n$-th power of an observable indicates that $\mathfrak{O}$ can be assigned more algebraic structure. It has been suggested to define a product

$$
A \circ B=\frac{1}{2}\left[(A+B)^{2}-A^{2}-B^{2}\right]
$$

for $A, B \in \mathfrak{O}$. This product is commutative but in general nonassociative. If $\circ$ is assumed to satisfy in addition that $A \circ\left(A^{2} \circ B\right)=A^{2} \circ(A \circ B)$ for 
all $A, B \in \mathfrak{O}$ (weak associativity), then it endows $\mathfrak{O}$ with the structure of a Jordan algebra, see $[10,28,31]$. We shall not pursue the Jordan algebra approach here. We only remark that it has been shown that, if it is augmented by two more topological assumptions, it can account for most of the quantum mechanical structure [28].

Instead we assume that $\mathfrak{O}$ can be embedded in a complex algebra $\mathfrak{A}$ such that $\operatorname{alg}_{\mathbb{C}} \mathfrak{O}=\mathfrak{A}$ where $\mathfrak{A}$ has an, in general, noncommutative product $A B$, where $A, B \in \mathfrak{A}$, and an antilinear involution denoted by *. (Then a Jordan algebra structure can be recovered by defining $A \circ B=\frac{1}{2}(A B+$ $B A$ ).) This assumption will cover all known physical situations. That is, $\mathfrak{A}$ is a complex vector space with an associative and distributive product and an involution such that

$$
(\lambda A+\mu B)^{*}=\bar{\lambda} A^{*}+\bar{\mu} B^{*}, \quad(A B)^{*}=B^{*} A^{*}
$$

for any $A, B \in \mathfrak{A}$ and $\lambda, \mu \in \mathbb{C}$. The states $\Sigma$ are extended to $\mathfrak{A}$ to yield a separating family of complex linear functionals on $\mathfrak{A}$. The natural extension of the property $\varphi\left(A^{2}\right) \geq 0$ is

$$
\varphi\left(A^{*} A\right) \geq 0, \quad \varphi \in \Sigma, A \in \mathfrak{A} .
$$

We can still define a norm on $\mathfrak{A}$ by $\|A\|=\sup \{|\varphi(A)|: \varphi \in \Sigma\}$, then we assume the generalization of (1.2),

$$
\left\|A^{*} A\right\|=\|A\|^{2}, \quad A \in \mathfrak{A},
$$

as well as

$$
\|A B\| \leq\|A\| \cdot\|B\|, \quad A, B \in \mathfrak{A} .
$$

By positivity we have $\varphi\left((\lambda A+\mathbb{1})^{*}(\lambda A+\mathbb{1})\right) \geq 0$, which implies that

$$
\varphi\left(A^{*}\right)=\overline{\varphi(A)}, \quad\left\|A^{*}\right\|=\|A\|,
$$

for any $A \in \mathfrak{A}$ and by a suitable choice of $\lambda \in \mathbb{C}$.

We see if we add the assumption that $\mathfrak{A}$ is complete in the norm $\|\cdot\|$ then $\mathfrak{A}$ satisfies all axioms of a $C^{*}$-algebra. Thus we will henceforth assume that $\mathfrak{A}$ is a $\mathrm{C}^{*}$-algebra and the observables correspond to the self-adjoint (i. e. $A=A^{*}$ ) elements of $\mathfrak{A}$ because for a self-adjoint $A \in \mathfrak{A}$ we have $\varphi(A) \in \mathbb{R}$ for any $\varphi \in \Sigma$ as a consequence of (1.7). The states $\Sigma$ are identified with the set of all continuous positive and normalized functionals on $\mathfrak{A}$, i. e. we have the identification

$$
\Sigma \cong\left\{\varphi \in \mathfrak{A}^{*}: \varphi\left(A^{*} A\right) \geq 0 \forall A \in \mathfrak{A}, \varphi(\mathbb{1})=1\right\}=: \mathscr{S}(\mathfrak{A}) .
$$


As we have already mentioned, given a state and an observble, the corresponding probability distribution can be found by the Hamburger moment problem, which is the following.

Proposition 1.1 Let $\left\{a_{k}\right\}_{k \in \mathbb{N}_{0}} \subseteq \mathbb{R}$ be a sequence. If for every $n \in \mathbb{N}$ and all $z_{1}, \ldots, z_{n} \in \mathbb{C}$ we have

$$
\sum_{k, \ell=0}^{n} \bar{z}_{k} z_{\ell} a_{k+\ell} \geq 0
$$

and if there exist $c, d \geq 0$ such that $\left|a_{k}\right| \leq c d^{k} k$ ! for every $k \in \mathbb{N}_{0}$ then there exists a unique positive Borel measure $\mu$ on $\mathbb{R}$ such that $\int_{\mathbb{R}} t^{k} \mathrm{~d} \mu(t)=a_{k}$ for every $k \in \mathbb{N}_{0}$

Given a state $\varphi$ and a self-adjoint element $A \in \mathfrak{A}$ we can easily verify that $a_{k}=\varphi\left(A^{k}\right)$ satisfies the conditions of the previous proposition: We have

$$
\sum_{k, \ell=1}^{n} \bar{z}_{k} z_{\ell} \varphi\left(A^{k+\ell}\right)=\varphi\left[\left(\sum_{k=1}^{n} z_{k} A^{k}\right)\left(\sum_{\ell=1}^{n} z_{\ell} A^{\ell}\right)^{*}\right] \geq 0
$$

as well as $\left|a_{k}\right| \leq\|A\|^{k}$. Thus the pair $\varphi$ and $A=A^{*}$ indeed induces a unique probability distribution on $\mathbb{R}$.

Because of this interpretation the $\mathrm{C}^{*}$-algebra $\mathfrak{A}$ is sometimes called the algebra of observables of a physical system. However, it has a more general interpretation beyond this heuristic construction of $\mathfrak{A}$, for this reason we prefer the name kinematical algebra because the role of $\mathfrak{A}$ is to encode the kinematical structure of a physical system. In quantum field theory when unobservable fields are used or when there are superselection rules present in the system one still can use algebras to describe the kinematics of physical systems, however, their elements need not be related in a simple way to the observables (i. e. the actual measurements that can be performed on the system in the laboratory) of the system, see [12] for a thorough discussion of these points.

\subsection{3. $\mathrm{C}^{*}$-Algebras and von Neumann Algebras}

Since $\mathrm{C}^{*}$ - and von Neumann algebras play a crucial role in the following, we will recall some basic facts about them. This subsection is not meant to be an exhaustive review of all results of operator algebra theory that are used in the present work, it mainly servers to clarify the connection of the previous subsections to quantum probability theory and to fix some 
notation. For an exposition of $\mathrm{C}^{*}$-algebra and von Neumann algebra theory we refer to $[24,30,23,10,6,4]$. Henceforth we will adopt the notation of operator algebra theory and denote the elements of operator algebras by $x, y, z, \ldots$ since they are regarded as the basic structures of the theory.

A $C^{*}$-algebra $\mathfrak{A}$ is a complex vector space which admits an associative and distributive product $x y$ for $x, y \in \mathfrak{A}$ which is not necessarily commutative, an antilinear involution $x \mapsto x^{*}$, that is

$$
\begin{aligned}
(\lambda x)^{*} & =\bar{\lambda} x^{*}, & (x+y)^{*} & =x^{*}+y^{*}, \\
\left(x^{*}\right)^{*} & =x, & (x y)^{*} & =x^{*} y^{*},
\end{aligned}
$$

and a norm $\|\cdot\|$ which makes $\mathfrak{A}$ a Banach algebra (so in particular $\mathfrak{A}$ is complete in the norm topology) and which moreover satisfies the Gelfand condition

$$
\left\|x^{*} x\right\|=\|x\|^{2}, \quad x \in \mathfrak{A} .
$$

If $\mathfrak{A}$ contains a unit $\mathbb{1}$ such that $\mathbb{1} x=x \mathbb{1}=x$ for any $x \in \mathfrak{A}$ then $\mathfrak{A}$ is called unital. As a rule all $\mathrm{C}^{*}$-algebras appearing in this work are unital (unless stated otherwise). An element $x \in \mathfrak{A}$ is called self-adjoint if $x^{*}=x$, the real vector space of all self-adjoint elements is denoted by $\mathfrak{A}^{\text {sa }}$. The space $\mathfrak{A}^{\text {sa }}$ carries a natural order: $x$ is called positive, notation $x \geq 0$, if $x=y^{*} y$ for some $y \in \mathfrak{A}$, the cone of all positive elements in $\mathfrak{A}$ is denoted by $\mathfrak{A}^{+}$. The spectrum of $x \in \mathfrak{A}$ is defined by

$$
\operatorname{spec} x=\{\lambda \in \mathbb{C}:(\lambda \mathbb{1}-x) \text { is not invertible in } \mathfrak{A}\} .
$$

A normal element $x \in \mathfrak{A}$, i.e. $x x^{*}=x^{*} x$, is self-adjoint if and only if spec $x \subseteq \mathbb{R}$, and positive if and only if $\operatorname{spec} x \subseteq \mathbb{R}^{+}$. Particularly important positive operators are the projections: $p \in \mathfrak{A}$ is called a projection if $p=$ $p^{2}=p^{*}$, the set of all projections is denoted by $\mathscr{P}(\mathfrak{A})$. Two projections $p, q \in \mathscr{P}(\mathfrak{A})$ are called orthogonal, in symbols $p \perp q$, if $p q=0$.

A state on a $\mathrm{C}^{*}$-algebra $\mathfrak{A}$ is a linear functional $\varphi$ on $\mathfrak{A}$ which is positive (i. e. $\varphi(x) \geq 0$ if $x \geq 0$ ) and normalized, i. e. its norm

$$
\|\varphi\|=\sup \{|\varphi(x)|: x \in \mathfrak{A},\|x\| \leq 1\}
$$

is unity, or equivalently $\varphi(\mathbb{1})=1$ if $\mathfrak{A}$ is unital. The set of all states on $\mathfrak{A}$ will be denoted by $\mathscr{S}(\mathfrak{A})$. A state $\varphi$ is called faithful if $x \geq 0$ and $\varphi(x)=0$ imply $x=0$. A state $\varphi$ is called pure if for any other state $\psi$ such that $\varphi \leq \psi$ it follows that $\varphi=\lambda \psi$ for some $\lambda \in[0,1]$, where $\varphi \leq \psi$ means $\varphi(x) \leq \psi(x)$ for all $x \in \mathfrak{A}^{+}$.

There are two important examples of $\mathrm{C}^{*}$-algebras. Let $\mathscr{H}$ be a Hilbert space and let $\mathrm{L}(\mathscr{H})$ denote the set of all bounded linear operators on $\mathscr{H}$. 
Under addition, scalar multiplication, and pointwise multiplication together with the involution $x \mapsto x^{*}$ given by taking adjoints of linear operators as well as the usual operator norm, $\mathrm{L}(\mathscr{H})$ is a $\mathrm{C}^{*}$-algebra. A *-invariant subalgebra $\mathfrak{A} \subseteq \mathrm{L}(\mathscr{H})$ is a $\mathrm{C}^{*}$-algebra as well provided that it is closed in the norm topology on $\mathrm{L}(\mathscr{H})$. We call a closed *-invariant subalgebra of $\mathrm{L}(\mathscr{H})$ a concrete $\mathrm{C}^{*}$-algebra. This example exhausts already the class of $\mathrm{C}^{*}$-algebras in the sense that it can be shown that every $\mathrm{C}^{*}$ algebra is isomorphic to some concrete $\mathrm{C}^{*}$-algebra on a suitable Hilbert space, which is the content of the important Gelfand-Naimark theorem. Since we have seen in Section 1.1.2 that (the kinematics of) general physical systems is described by $\mathrm{C}^{*}$-algebras this shows that in this way we enter the Hilbert space and operator framework familiar from elementary quantum mechanics. We shall elaborate on this point below.

The second important example is provided by the set of all complex continuous functions $\mathrm{C}_{0}(\Omega)$ vanishing at infinity on a locally compact Hausdorff topological space $\Omega$. This set becomes a $\mathrm{C}^{*}$-algebra under the pointwise product $(f g)(\omega):=f(\omega) g(\omega)$, where $\omega \in \Omega$ and $f, g \in \mathrm{C}_{0}(\Omega)$, and involution defined by complex conjugation, $f^{*}:=\bar{f}$. The norm on $\mathrm{C}_{0}(\Omega)$ is taken to be the sup-norm, i.e. $\|f\|=\sup \{|f(\omega)|: \omega \in \Omega\}$. Again, this example exhausts the class of all commutative $\mathrm{C}^{*}$-algebras up to isomorphism for suitable locally compact Hausdorff spaces $\Omega$. This fact will be used and explained in more detail in the next subsection. We remark that $\Omega$ is compact if and only if $\mathfrak{A}$ is unital.

Besides $\mathrm{C}^{*}$-algebras another class of algebras are important for our purpose. A von Neumann algebra is a concrete $\mathrm{C}^{*}$-algebra $\mathfrak{M}$ contained in $\mathrm{L}(\mathscr{H})$ for some Hilbert space $\mathscr{H}$ which is closed in the strong operator topology. That is, whenever $\left\{x_{i}\right\}_{i \in I}$ is a net of elements of $\mathfrak{M}$ such that it converges to $x \in \mathrm{L}(\mathscr{H})$ in the strong operator topology, i. e. $\left\|x_{i} \xi-x \xi\right\| \rightarrow 0$ as $i \rightarrow \infty$ for any vector $\xi \in \mathscr{H}$, then it follows that $x \in \mathfrak{M}$. Von Neumann algebras can also be characterized by a purely algebraic property. Let $M \subseteq \mathrm{L}(\mathscr{H})$ be a subset, then we define its commutant by $M^{\prime}=\{x \in \mathrm{L}(\mathscr{H}): x y=y x$ for all $y \in M\}$. Then a $\mathrm{C}^{*}$-subalgebra $\mathfrak{M} \subseteq \mathrm{L}(\mathscr{H})$ acting nondegenerately on $\mathscr{H}$, i. e. $\overline{\operatorname{lin}} \mathfrak{M} \mathscr{H}=\mathscr{H}$ (in particular, this is the case if $\mathfrak{M}$ containins the identity operator $\mathbb{1}$ ) is a von Neumann algebra if and only if $\mathfrak{M}^{\prime \prime}=\mathfrak{M}$. Finally, a von Neumann algebra may be characterized as a concrete $\mathrm{C}^{*}$-algebra $\mathfrak{M}$ which is the dual (in the Banach space sense) of a Banach space $\mathfrak{M}_{*}$, called the predual space of $\mathfrak{M}$, that is $\left(\mathfrak{M}_{*}\right)^{*}=\mathfrak{M}$. The predual can be shown to be uniquely determined. Then we have the inclusion of $\mathfrak{M}_{*}$ in the dual space $\mathfrak{M}^{*}$, i. e. the space of all continuous linear functionals on $\mathfrak{M}$. Any state on $\mathfrak{M}$ is an element of $\mathfrak{M}^{*}$; if it is also contained in $\mathfrak{M}_{*}$ we call the state normal. 
Normal states can be characterized by a continuity property. A state $\varphi$ is normal if and only if for any uniformly bounded increasing net $\left\{x_{i}\right\}_{i \in I}$ of $\mathfrak{M}^{+}$we have $\sup _{i \in I} \varphi\left(x_{i}\right)=\varphi\left(\sup _{i \in I} x_{i}\right)$, the sup exists (see [24]), or equivalently, if

$$
\varphi\left(\sum_{i \in I} p_{i}\right)=\sum_{i \in I} \varphi\left(p_{n}\right)
$$

for any family $\left\{p_{i}\right\}_{i \in I}$ of mutually orthogonal (i.e. $p_{i} \perp p_{j}$ if $i \neq j$ ) projections in $\mathscr{P}(\mathfrak{M})$. Finally it can be shown that for a normal state $\varphi$ on $\mathfrak{M}$ there exists a positive trace class operator $\rho$ on $\mathscr{H}$ with unit trace such that $\varphi(x)=\operatorname{tr} \rho x$ for any $x \in \mathfrak{M}$; in quantum mechanics such a $\rho$ is called a density matrix. Unlike a $\mathrm{C}^{*}$-algebra, which in the extreme case contains no projections except 0 , a von Neumann algebra always has plenty of projections. In fact, $\mathscr{P}(\mathfrak{M})$ is an orthomodular lattice and the set of all projections generates the algebra in the sense that $\mathscr{P}(\mathfrak{M})^{\prime \prime}=\mathfrak{M}$. For two projections $p, q \in \mathscr{P}(\mathfrak{M})$ we denote by $p \vee q$ and $p \wedge q$ the supremum and infimum, respectively, of $p$ and $q$ in $\mathscr{P}(\mathfrak{M})$. Given a self-adjoint element $x \in \mathfrak{M}$ with a pure point spectrum $\operatorname{spec} A$ we can write its spectral resolution as

$$
x=\sum_{\lambda \in \operatorname{spec} x} \lambda p_{\lambda},
$$

where $p_{\lambda}$ is the projection onto the space of all eigenvectors with eigenvalue $\lambda$. One can show that then $p_{\lambda} \in \mathfrak{M}$ for any $\lambda \in \operatorname{spec} A$. More generally, if $x$ is a self-adjoint element in $\mathfrak{M}$ its spectrum $\operatorname{spec} A$ need not be discrete and its spectral resolution becomes a continuous version of the above,

$$
x=\int_{\operatorname{spec} x} \lambda \mathrm{d} P(\lambda)
$$

where $P$ is now a projection-valued measure on $\operatorname{spec} A$, and again it can be shown that $P$ takes values in $\mathscr{P}(\mathfrak{M})$.

If $\mathfrak{M}$ is a von Neumann algebra, $\varphi$ a normal state and $x \in \mathfrak{M}$ is selfadjoint, then by Proposition 1.1 the sequence $\left\{\varphi\left(x^{k}\right)\right\}_{k \in \mathbb{N}_{0}}$ defines a unique probability measure $\mu_{\varphi}$ on $\mathbb{R}$ such that $\int_{\mathbb{R}} t^{k} \mathrm{~d} \mu_{\varphi}(t)=\varphi\left(x^{k}\right)$. Let $P$ be the spectral measure of $x$, then it follows that $\mu_{\varphi}$ concides with the probability measure $\varphi(P(\cdot))$, for it is easily seen by normality that $\mu_{\varphi}(B)=\varphi(P(B))$ for all Borel sets $B \subseteq \mathbb{R}$. Thus starting from an algebraic approach to quantum theory as in Section 1.1.2 with a direct physical interpretation of the expectation values $\varphi(x)$ for observables $x$ and states $\varphi$, we recover the usual interpretation of the quantum physical formulation: The measure $\mu_{\varphi}$ is concentrated on $\operatorname{spec} x$, i. e. the spectrum of $x$ is the set of all possible 
outcomes of the observable and the probability distribution is found by spectral resolution of $x$.

Let $(\Omega, \mathscr{F}, P)$ be a classical probability space. Denote by $\mathrm{L}^{\infty}(\Omega, P)$ the set of all $\mathscr{F}$-measurable (essentially) bounded functions on $\Omega$. Then endowed with the (essential) sup-norm, the pointwise product and the involution given by complex conjugation $\mathrm{L}^{\infty}(\Omega, P)$ is a $\mathrm{C}^{*}$-algebra. It is in fact a concrete $\mathrm{C}^{*}$-algebra on the Hilbert space $\mathrm{L}^{2}(\Omega, P)$ which acts on $\mathrm{L}^{2}(\Omega, P)$ by pointwise multiplication. Since $\mathrm{L}^{\infty}(\Omega, P)$ is the dual of the Banach space $\mathrm{L}^{1}(\Omega, P)$ of all measurable $P$-integrable functions (that is, all random variables on $(\Omega, \mathscr{F}, P)$ with finite expectation value), $\mathrm{L}^{\infty}(\Omega, P)$ is a von Neumann algebra. Clearly, it is commutative. The projections $p \in \mathscr{P}\left(\mathrm{L}^{\infty}(\Omega, P)\right)$ are precisely the characteristic functions $\chi_{E}$ where $E$ runs through $\mathscr{F}$, here

$$
\chi_{E}(\omega)=\left\{\begin{array}{ll}
1: & \omega \in E \\
0: & \omega \notin E
\end{array}, \quad \omega \in \Omega .\right.
$$

We shall need an number of different topologies on $\mathrm{L}(\mathscr{H})$. For an account on this subject see $[4,6,24]$. The most important topology is the ultraweak topology, it is the locally convex topology on $\mathrm{L}(\mathscr{H})$ defined by the family of seminorms $x \mapsto \operatorname{tr}(\rho x)$, where $\rho \in \mathrm{T}(\mathscr{H})=(\mathrm{L}(\mathscr{H}))_{*}$ (the predual). On the unit ball of $\mathrm{L}(\mathscr{H})$ it agrees with the weak operator topology, which is defined by the family of seminorms $x \mapsto\langle\eta, x \xi\rangle$, where $\xi, \eta$ run through $\mathscr{H}$. It is an important result (see e. g. [24]) that for any von Neumann algebra $\mathfrak{M} \subseteq \mathrm{L}(\mathscr{H})$ the relative ultraweak topology on $\mathfrak{M}$ agrees with the $\sigma\left(\mathfrak{M}, \mathfrak{M}_{*}\right)$-topology. Any positive normal linear functional on a von Neumann algebra $\mathfrak{M} \subseteq \mathrm{L}(\mathscr{H})$ is normal if and only if it is $\sigma\left(\mathfrak{M}, \mathfrak{M}_{*}\right)$-continuous. A bounded linear operator $T: \mathfrak{M} \longrightarrow \mathfrak{M}$ is called normal if it is positive, i. e. $T\left(\mathfrak{M}^{+}\right) \subseteq \mathfrak{M}^{+}$, and $\sigma\left(\mathfrak{M}, \mathfrak{M}_{*}\right)-\sigma\left(\mathfrak{M}, \mathfrak{M}_{*}\right)$ continuous. This is equivalent to $T^{*}\left(\mathfrak{M}_{*}\right) \subseteq \mathfrak{M}_{*}$, where $T^{*}: \mathfrak{M}^{*} \longrightarrow$ $\mathfrak{M}^{*}$ is the dual of $T$. I. e. in this case we can define a predual map $T_{*}$ by restricting $T^{*}$ to $\mathfrak{M}_{*} \subseteq \mathfrak{M}^{*}$. Moreover, normality of $T$ is equivalent to the normality of the positive functional $\varphi \circ T$ for all normal positive functional $\varphi$.

\subsection{Quantum Probability and Physical Systems}

After we have motivated the description of physical systems by $\mathrm{C}^{*}$-algebras we now show that classical probability also has a place in an algebraic 
framework. This formulation admits a generalization of classical probability theory to the so-called quantum probability theory. Quantum probability theory will be seen to be an adequate tool for the description of general quantum systems.

\subsubsection{Algebraic Classical Probability}

The basic principles of classical probability theory will not be recalled here, see e.g. [16], an explanation of the standard notation we will use in the sequel can also be found there. We only remind the reader that a classical probability space is a triple $(\Omega, \mathscr{F}, P)$ consinsting of a set $\Omega$, the sample space, a $\sigma$-algebra of subsets $\mathscr{F}$ of $\Omega$, and a $\sigma$-additive map $P: \mathscr{F} \longrightarrow[0,1]$ such that $P(\Omega)=1$, the probability distribution. The points of the sample space $\Omega$ represent the outcomes of a random experiment, a subset $E \subseteq \Omega$ lying in $\mathscr{F}$ is called an event; if $\omega \in \Omega$ is the outcome of a run of the random experiment and $\omega \in E$, then we say that the event $E$ has taken place. The number $P(E)$ is called the probability of $E$.

Our purpose is to formulate the classical probability model in an algebraic language by associating with every classical probability space a commutative von Neumann algebra plus some extra structure, which may be described axiomatically. If we then drop the commutativity assumption from the list of axioms we obtain a generalization of classical probability. This generalization turns out to be just right for quantum theory and it becomes apparent that quantum theory is best viewed as a generalization of classical probability theory. The method of passing from a commutative to a noncommutative algebra is in line with a general theme of modern mathematics: To obtain a noncommutative or "quantum" generalization of some mathematical structure one casts the axioms of the structure in terms of properties of an appropriate commutative algebra of functions which carries some extra structure. Then one generalizes from commutative to noncommutative algebras to obtain the quantum version. This procedure has been successfully applied to probability (see the following), topology $\left(\mathrm{C}^{*}\right.$-algebras may be viewed as a noncommutative version of topological spaces), groups (generalized by quantum groups), and differential geometry.

First we briefly review Gelfand's theory of commutative $\mathrm{C}^{*}$-algebras. Let $\mathfrak{A}$ be a commutative $\mathrm{C}^{*}$-algebra, then a character $\omega$ is a state on $\mathfrak{A}$ such that $\omega(x y)=\omega(x) \omega(y)$ for all $x, y \in \mathfrak{A}$. Let $\Omega(\mathfrak{A})$ be the set of all characters on $\mathfrak{A}$. Since each character is contained in the dual $\mathfrak{A}^{*}$ of $\mathfrak{A}$, the set $\Omega(\mathfrak{A})$ can be given the relative weak* topology inherited from $\mathfrak{A}^{*}$. Now Gelfand's theorem says that with this topology $\Omega(\mathfrak{A})$ is a locally compact 
Hausdorff space (which is even compact if $\mathfrak{A}$ is unital) and that the map $\mathfrak{A} \longrightarrow \mathrm{C}_{0}(\Omega(\mathfrak{A}))$ given by $x \mapsto \hat{x}$, where $\hat{x}$ is defined by $\hat{x}(\omega)=\omega(x)$ for any character $\omega \in \Omega(\mathfrak{A})$, is an isometric isomorphism of $\mathfrak{A}$ onto $\mathrm{C}_{0}(\Omega(\mathfrak{A}))$; it is called the Gelfand isomorphism. Thus these two $\mathrm{C}^{*}$-algebras may be identified via the Gelfand isomorphism.

Besides Gelfand's theorem we shall also need the important GelfandNaimark-Segal (GNS) theorem which associates to every $\mathrm{C}^{*}$-algebra $\mathfrak{A}$ and every state $\varphi$ a representation of $\mathfrak{A}$ acting on a Hilbert space.

Theorem 1.2 Let $\mathfrak{A}$ be a $C^{*}$-algebra and $\varphi$ a state on $\mathfrak{A}$. Then there exists a Hilbert space $\mathscr{H}_{\varphi}$ and $a{ }^{*}$-representation $\pi_{\omega}: \mathfrak{A} \longrightarrow \mathrm{L}\left(\mathscr{H}_{\varphi}\right)$, and a normalized vector $\xi_{\varphi} \in \mathscr{H}_{\varphi}$, such that

$$
\varphi(x)=\left\langle\xi_{\phi}, \pi_{\varphi}(x) \xi_{\varphi}\right\rangle, \quad x \in \mathfrak{A},
$$

and such that $\xi_{\varphi}$ is cyclic for $\pi_{\varphi}$, i.e. $\overline{\pi_{\varphi}(\mathfrak{A}) \xi_{\varphi}}=\mathscr{H}_{\varphi}$. The representation $\pi_{\varphi}$ on $\mathscr{H}_{\varphi}$ is uniquely determined by $\varphi$ up to unitary equivalence.

A proof can be found, e.g. in $[4,6,23,24]$. Given a $\mathrm{C}^{*}$-algebra $\mathfrak{A}$ and a state $\varphi$ we will the canonical objects associated to $\varphi$ denote by the triplet $\left(\pi_{\varphi}, \xi_{\varphi}, \mathscr{H}_{\varphi}\right)$.

The following result is the basic ingredient in describing a classical probability space in algebraic terms.

Proposition 1.3 Let $(\Omega, \mathscr{F}, P)$ be a classical probability space. Then $\mathfrak{M}=\mathrm{L}^{\infty}(\Omega, P)$ is a commutative von Neumann algebra acting on $\mathscr{H}=$ $\mathrm{L}^{2}(\Omega, P)$, and

$$
\mathfrak{M} \ni x \mapsto \varphi(x)=\int_{\Omega} x(\omega) \mathrm{d} P(\omega)
$$

is a faithful normal state on $\mathfrak{M}$. Conversely, given a commutative von Neumann algebra $\mathfrak{M}$ and a faithful normal state $\varphi$ on $\mathfrak{M}$ then there exists a classical probability space $(\Omega, \mathscr{F}, P)$ such that $\mathfrak{M}$ is isomorphic to $\mathrm{L}^{\infty}(\Omega, P)$ and $\varphi$ is given by (1.14).

Proof. Clearly, if $\varphi$ is defined by (1.14) then $\varphi(1)=1, \varphi\left(x^{*}, x\right) \geq 0$ for all $x \in \mathfrak{M}$, if $\varphi\left(x^{*} x\right)=0$ then $x(\omega)=0 P$-a. e., and $\varphi$ is normal. Conversely, if $\mathfrak{M}$ is commutative then $\mathfrak{M} \cong \mathrm{C}_{0}(\Omega)$ (in the $\mathrm{C}^{*}$-sense) for a locally compact Hausdorff space $\Omega$. Let $\mathscr{F}$ be the Borel $\sigma$-algebra on $\Omega$. By the Riesz-Markov theorem there exists a Radon probability measure $P$ on $(\Omega, \mathscr{F})$ such that $\varphi(x)=\int x(\omega) \mathrm{d} P(\omega)$ for all $x \in \mathrm{C}_{0}(\Omega)$. The GNS representation of $\mathfrak{M} \cong \mathrm{C}_{0}(\Omega)$ with respect to $\varphi$ is given by $\mathscr{H}_{\varphi}=\mathrm{L}^{2}(\Omega, P)$, $\xi_{\varphi}(\omega)=1$ for all $\omega \in \Omega$, and $\left(\pi_{\varphi}(x) \xi\right)(\omega)=x(\omega) \xi(\omega)$ for all $x \in \mathrm{C}_{0}(\Omega)$ 


\begin{tabular}{ll} 
Classical & Algebraic \\
\hline random variables & self-adjoint elements of $\mathfrak{M}$ \\
events $\mathscr{F}$ & projections $\mathscr{P}(\mathfrak{M})$ \\
probability distribution $P$ & state $\varphi$ \\
$\sigma$-additivity of $P$ & normality of $\varphi$ \\
inclusion (implication), $E_{1} \subseteq E_{2}$ & ordering of projections, $p_{1} \leq p_{2}$ \\
exclusive events, $E_{1} \cap E_{2}=\emptyset$ & orthogonality, $p_{1} \perp p_{2}$ \\
$E_{1} \cup E_{2}$ if $E_{1} \cap E_{2}=\emptyset$ & $p_{1} \vee p_{2}=p_{1}+p_{2}$ if $p_{1} \perp p_{2}$ \\
union, $E_{1} \cup E_{2}$ & supremum, $p_{1} \vee p_{2}$ \\
conditional expectation & noncomm. conditional expectation
\end{tabular}

Table 1.1.: Correspondence between some concepts of classical and algebraic probability. The concept of a noncommutative conditional expectation is introduced in Section 1.3.1 in connection with the dynamics of subsystems.

and $\xi \in \mathscr{H}_{\varphi}$. Now since $\varphi$ is normal and faithful, $\pi_{\varphi}: \mathfrak{M} \longrightarrow \pi_{\varphi}(\mathfrak{M})$ is normal and faithful, and $\pi_{\varphi}(\mathfrak{M}) \subseteq \mathrm{L}\left(\mathscr{H}_{\varphi}\right)$ is ultraweakly closed. The von Neumann algebra $\pi_{\varphi}\left(\mathrm{C}_{0}(\Omega)\right)$ is commutative and has a cyclic vector, hence it is maximal commutative. Since $\mathrm{L}^{\infty}(\Omega, P) \subseteq \pi_{\varphi}\left(\mathrm{C}_{0}(\Omega)\right)^{\prime}$ as well as $\pi_{\varphi}\left(\mathrm{C}_{0}(\Omega)\right) \subseteq \mathrm{L}^{\infty}(\Omega, P)$ this implies $\mathrm{L}^{\infty}(\Omega, P)=\pi_{\varphi}\left(\mathrm{C}_{0}(\Omega)\right)^{\prime \prime}=\pi_{\varphi}(\mathfrak{M})$ and hence $\mathfrak{M} \cong \mathrm{L}^{\infty}(\Omega, P)$ in the von Neumann algebra sense.

Thus every classical probability model leads to a commutative von Neumann algebra together with a faithful normal state in a canonical way, and every commutative von Neumann algebra $\mathfrak{M}$ together with a faithful normal state leads us back to a classical probability model.

\subsubsection{The Quantum Generalization}

In the last section we have obtained a description of a classical probability space in terms of commutative von Neumann algebras. Now we generalize this description to noncommutative algebras.

Definition 1.4 A pair $(\mathfrak{M}, \varphi)$ consisting of a von Neumann algebra $\mathfrak{M}$ and a normal state $\varphi$ on $\mathfrak{M}$ is called a quantum (or noncommutative) probability space.

We shall not introduce the concepts of quantum probability at a formal level in the present work but refer instead to the textbooks [17, 22] and [112]. But we shall try point out its relevance for the description of quantum systems. Indeed, from the probabilistic prespective we shall see that qunatum physics is a generalization of classical probability theory 
and not so much a generalization of classical mechanics or field theory. We propose that for the discussion of certain foundational issues of quantum mechanics like measurements and the emergence of classicality in a quantum world it is helpful to assume this point of view and use the algebraic formulation.

Our task is to identify the algebraic counterparts of some concepts of classical probability. To this end we use the relation between a classical probability space $(\Omega, \mathscr{F}, P)$ and the corresponding quantum probability space $(\mathfrak{M}, \varphi)$ with $\mathfrak{M}=\mathrm{L}^{\infty}(\Omega, P)$ given by Proposition 1.3. Clearly, the random variables (i.e. the observables of the random experiment) in the classical case become associated to the self-adjoint elements of the algebra, and we have already seen that the indicator random variables $\chi_{E}$, where $E \in \mathscr{F}$, correspond to the projections in $\mathrm{L}^{\infty}(\Omega, P)$. Thus the events $\mathscr{F}$ correspond to the projections $\mathscr{P}(\mathfrak{M})$. The probability distribution in the classical case becomes the state in the quantum case, where $\sigma$-additivity of $P$ corresponds to the normality of the state. This is clear in view of (1.10) and the association of events and projections. Similarly, we see that for two events $E_{1}, E_{2}$ of $\mathscr{F}$ the relation $E_{1} \subseteq E_{2}$ (i.e. the event $E_{1}$ implies $E_{2}$ ) corresponds to the ordering $p_{1} \leq p_{2}$ of projections, and $E_{1} \cap E_{2}=\emptyset$ corresponds to orthogonality $p_{1} \perp p_{2}$, finally the event " $E_{1}$ and $E_{2}$ ", i.e. $E_{1} \cup E_{2}$, corresponds to $p_{1} \vee p_{2}$. These generalizations are summarized in Table 1.1. A new trait in the noncommutative case is that we can have $\left[p_{1}, p_{2}\right]:=p_{1} p_{2}-p_{2} p_{1} \neq 0$ for $p_{1}, p_{2} \in \mathscr{P}(\mathfrak{M})$; this noncommutativity of projections has no counterpart in classical probability theory. If $\left[p_{1}, p_{2}\right]=0$ we say that the two projections are compatible or noninterfering. A similar terminology is adopted for general self-adjoint elements of $\mathfrak{M}$. Thus in the commutative case random variables are always compatible. Some consequences which noncommutativity of observables entails will be discussed in the next section after we have discussed the relation of quantum probability spaces to classical and quantum systems.

\subsubsection{Classical Physical Systems}

A classical mechanical system is described by a configuration space, given by a smooth finite-dimensional manifold $M^{m}$ of dimension $m$. A motion is a smooth curve $\gamma:] a, b\left[\longrightarrow M^{m}\right.$. In Hamiltonian mechanics the cotangent bundle $\mathrm{T}^{*} M^{m}$ is called the phase space of the system under study; the tangent of $\gamma, \dot{\gamma}:] a, b\left[\longrightarrow \mathrm{T} M^{m}\right.$, is interpreted as velocity. In the simplest case $\mathrm{T}^{*} M^{m}$ has the interpretation of being the space of generalized coordinates $q_{1}, \ldots, q_{m}$ and generalized momenta $p_{1}, \ldots, p_{m}$, where $\left(q_{1}, \ldots, q_{m}, p_{1}, \ldots, p_{m}\right)$ then is a point in phase space. Time evolution is 
specified by a flow $g: \mathbb{R} \times \mathrm{T}^{*} M^{m} \longrightarrow \mathrm{T}^{*} M^{m}$, so for every initial point $\omega \in \mathrm{T}^{*} M^{m}$ we obtain a smooth curve $\gamma_{t}(\omega)=g(t, \omega)$ for all $t \in \mathbb{R}$ which is the motion of the system in phase space. Here $\{g(t, \cdot)\}_{t \in \mathbb{R}}$ is a group of diffeomorphisms on $\mathrm{T}^{*} M^{m}$. The flow $g$ is determined by the Hamiltonian function $H: \mathrm{T}^{*} M^{m} \longrightarrow \mathbb{R}$ where $g$ gives the integral curves of the symplectic gradient s-grad $H$ on $\mathrm{T}^{*} M^{m}$ with respect to the canonical symplectic structure on $\mathrm{T}^{*} M^{m}$. In the simplest case when there are coordinates $\left(q_{1}, \ldots, q_{m}, p_{1}, \ldots, p_{m}\right)$ such that the canonical symplectic form can be written as $\sum \mathrm{d} p_{i} \wedge \mathrm{d} q_{i}$ the integral curves are determined by Hamilton's equations,

$$
\dot{q}_{i}=\frac{\partial H}{\partial p_{i}}, \quad \dot{p}_{i}=-\frac{\partial H}{\partial q_{i}}, \quad i=1, \ldots, m .
$$

In classical mechanics it is argued that phase space $\mathrm{T}^{*} M^{m}$ corresponds to the state space of the system and that an observable is a function $f: \mathrm{T}^{*} M^{m} \longrightarrow \mathbb{R}$. Thus as $\mathrm{C}^{*}$-algebra of a classical Hamiltonian system we take $\mathfrak{A}=\mathrm{C}_{0}(\Omega)$, where $\Omega=\mathrm{T}^{*} M^{m}$, i. e. the algebra of all complex continuous functions on $\Omega$ vanishing at infinity. Then the self-adjoint elements of $\mathfrak{A}$ (i. e. real valued functions) are the observables. By the Riesz-Markov theorem the dual of $\mathrm{C}_{0}(\Omega)$ is the space of all finite Radon measures on $\Omega$, hence the state space $\mathscr{S}(\mathfrak{A})$ is the set $\mathscr{M}_{1}^{+}(\Omega)$ of all probability Radon measures on phase space $\Omega$. Thus for a state $\mu \in \mathscr{M}_{1}^{+}(\Omega)$ and observable $f$ the expectation value is given by

$$
\mu(f)=\int_{\Omega} f(\omega) \mathrm{d} \mu(\omega) .
$$

In particular, an observable $f$ can be regarded as a random variable on the probability space $(\Omega, \mathscr{B}, \mu)$. The pure states are precisely the Dirac measures $\delta_{\omega}$ concentrated in a single point $\omega$ of phase space $\Omega$. They have the property of being dispersion free, i. e. if $\mu=\delta_{\omega}$ the variance vanishes, $\operatorname{Var} f=\int_{\Omega}(f-\mu(f))^{2} \mathrm{~d} \delta_{\omega}=0$, and any observable $f \in \mathfrak{A}$ takes on a sharp value $f(\omega)$. Thus if the system is in a pure state we know the value of each observable exactly. If the system is in a nonpure state we only have a probability distribution on phase space. This is an entirely classical probability distribution and its presence may be interpreted as lack of knowledge in which state the system actually is. So probabilities play here the role of subjective ignorance, albeit this role may be very significant, for example in statistical mechanics: We are not able to practically solve a coupled system of $10^{23}$ differential equations, nor are we able to even store and process the amount of data present in the solutions, which renders such 
an approach useless. Instead, we content ourselves with a probabilistic description. In fact, this is what is appropriate in this situation, since, after all, we are only interested in certain macroscopic properties of systems in statistical mechanics, such as temperature, pressure, volume, magnetization, etc., hence it is inappropriate to describe them as $N$-particle systems with $N \approx 10^{23}$.

Time evolution translates into the algebraic framework as follows: The flow $g$ induces a group of $*_{\text {-automorphisms }}\left\{\alpha_{t}\right\}_{t \in \mathbb{R}}$ on $\mathfrak{A}$ via

$$
\alpha_{t}(f)(\omega)=f(g(t, \omega)), \quad t \in \mathbb{R}, \omega \in \Omega, f \in \mathfrak{A} .
$$

The fact that $\left\{\alpha_{t}\right\}_{t \in \mathbb{R}}$ is a group of automorphism reflects the fact that time evolution is reversible. The appearance of irreversible time evolution is characteristic for open systems.

\subsubsection{Quantum Systems}

In Section 1.1.2 we have argued that any physical system can be described in terms of $\mathrm{C}^{*}$-algebras. Let us consider an arbitrary physical system which is described by some $\mathrm{C}^{*}$-algebra $\mathfrak{A}$. Then we have the famous Heisenberg uncertainty relation in its general form:

Lemma 1.5 Let $\mathfrak{A}$ be a $C^{*}$-algebra and $x, y \in \mathfrak{A}$ two self-adjoint elements. For any state $\varphi$ on $\mathfrak{A}$ write $\Delta_{\varphi}(x)=\varphi\left[(x-\varphi(x))^{2}\right]$ for the variance of $x$ in the state $\varphi$, and similarly for $y$. Then

$$
\Delta_{\varphi}(x) \Delta_{\varphi}(y) \geq \frac{1}{2}|\varphi([x, y])| .
$$

Proof. From the Cauchy-Schwarz inequality $|\varphi(x y)| \leq \varphi\left(x^{2}\right) \varphi\left(y^{2}\right)$ we immediately get $\frac{1}{2}|\varphi(x y-y x)| \leq \varphi\left(x^{2}\right) \varphi\left(y^{2}\right)$. Replacing $x$ and $y$ with the self-adjoint operators $x-\varphi(x)$ and $y-\varphi(y)$ this implies $\frac{1}{2} \mid \varphi([x-\varphi(x), y-$ $\varphi(y)])\left|=\frac{1}{2}\right| \varphi([x, y]) \mid \leq \Delta_{\varphi}(x) \Delta_{\varphi}(y)$.

Hence we see that for a commutative $\mathfrak{A}$ the right hand side of (1.16) is always trivial and there is no Heisenberg uncertainty principle. It was argued by Heisenberg that, as a matter of principle, when we measure the position of a particle of atomic dimensions we perturb the particle's momentum, and that the perturbation becomes larger the more accurate we try to determine its position, no matter in which state $\varphi$ the particle is, so that an inequality of the form (1.16) for the position and momentum observables is expected to hold for any state $\varphi$. Thus we can guess from Lemma 1.5 that the presence of quantum properties of a system, embodied by the uncertainty principle, entails that its algebra is noncommutative. 
The starting point of quantum mechanics, motivated by the heuristic uncertainty principle, is the assumption that the canonical variables $q_{1}, \ldots, q_{n}, p_{1}, \ldots, p_{n}$ describing a physical system no longer commute as in the classical case but satisfy the Heisenberg canonical commutation relations,

$$
\left[q_{i}, p_{j}\right]=\mathrm{i} \delta_{i j}, \quad\left[q_{i}, q_{j}\right]=0, \quad\left[p_{i}, p_{j}\right]=0,
$$

for $i, j=1, \ldots, n$. This procedure is called canonical quantization. Since these relations entail that the $q_{1}, \ldots, q_{n}, p_{1}, \ldots, p_{n}$ cannot be represented by bounded operators on a Hilbert space one usually considers bounded functions of them, for example $u_{t}^{(i)}(t)=\mathrm{e}^{\mathrm{i} t q_{i}}, v_{t}^{(j)}=\mathrm{e}^{\mathrm{i} t p_{i}}, i=1, \ldots, n$, $t \in \mathbb{R}$. This leads to the CCR algebra, studied in Appendix A, which is the relevant $\mathrm{C}^{*}$-algebra in this case. Roughly, it is the $\mathrm{C}^{*}$-algebra generated by the $u_{t}^{(i)}(t)$ and $v_{t}^{(i)}(t)$, where $i=1, \ldots, n$ and $t \in \mathbb{R}$. Thus it contains all observables which are bounded functions of the operators $q_{1}, \ldots, q_{n}, p_{1}, \ldots, p_{n}$. For example, for a single particle moving in one dimension we choose (in the notation of Appendix A) the symplectic space $\mathbf{S}=\mathbb{R}^{2}$ with the canonical symplectic form $\sigma\left(\left(x_{1}, x_{2}\right),\left(y_{1}, y_{2}\right)\right)=$ $x_{1} y_{2}-x_{2} y_{1}$. Then if $u_{t}=\mathrm{e}^{\mathrm{i} t q}$ and $v_{t}=\mathrm{e}^{\mathrm{i} t p}, t \in \mathbb{R}$, are the unitary groups generated by the position operator $q$ and momentum operator $p$ acting on the Hilbert space $\mathscr{H}=\mathrm{L}^{2}(\mathbb{R})$, then $w\left(x_{1}, y_{1}\right)=\mathrm{e}^{-x_{1} x_{1} / 2} v_{x_{2}} u_{x_{1}}$ defines a Weyl system (see page 149), which is by the Stone-von Neumann theorem (Theorem A.16) unique up to unitary equivalence. By Stone's theorem and differentiation the Weyl form of the canonical commutation relations (A.5) leads back to the familiar Heisenberg canonical commutation relations (1.17). In this way we see that in standard quantum mechanics for a single particle (more generally, for $n$ particles) leads to a noncommutative $\mathrm{C}^{*}$-algebra, in line with our above guess about the noncommutativity of the $\mathrm{C}^{*}$-algebra describing a quantum system.

Let $\mathfrak{A}$ be a $\mathrm{C}^{*}$-algebra and $\varphi$ a state on $\mathfrak{A}$. According to the GNS theorem, Theorem 1.2, there exists a representation $\pi_{\varphi}$ on a Hilbert space $\mathscr{H}_{\varphi}$ with cyclic vector $\xi_{\varphi} \in \mathscr{H}_{\varphi}$, i.e. $\mathfrak{A}$ is represented as a concrete $\mathrm{C}^{*}$ algebra $^{2} \pi_{\omega}(\mathfrak{A})$ acting on $\mathscr{H}_{\varphi}$ in such a way that $\varphi$ becomes the vector state $\left\langle\xi_{\varphi}, \xi_{\varphi}\right\rangle$. In this setting, by the cyclicity of $\xi_{\varphi}$, the set of all vector states $\pi_{\varphi}(\mathfrak{A}) \ni x \mapsto\langle\xi, x \xi\rangle$ for some normalized vector $\xi \in \mathscr{H}_{\varphi}$ is interpreted as the set of states which can be prepared by instruments described by the algebra $\pi_{\varphi}(\mathfrak{A})$. More generally, we can allow mixtures of vector states and consider arbitrary convex combinations of vector states on $\pi_{\varphi}(\mathfrak{A})$, again with the interpretation that they are constructed by instruments from $\pi_{\varphi}(\mathfrak{A})$ and mixing. Thus it seems natural to close the

${ }^{2}$ It can be shown that $\pi_{\varphi}(\mathfrak{A})$ is indeed a $\mathrm{C}^{*}$-algebra. 
concrete $\mathrm{C}^{*}$-algebra $\pi_{\varphi}(\mathfrak{A})$ in a topology where convergence is equivalent to the convergence of all vector states, or all convex combinations of vector states. This topology is the weak operator topology in the former case and the ultraweak topology in the latter case. The closure in both topologies agrees and by von Neumann's bicommutant theorem it is the $\mathrm{C}^{*}$-algebra generated by $\pi_{\varphi}(\mathfrak{A})$, which we denote as

$$
\mathfrak{M}=\pi_{\varphi}(\mathfrak{A})^{\prime \prime}
$$

In the above example of a quantum particle in one dimension (more generally, $n$ particles in $d$ dimensions) we find that $\mathfrak{M}=\mathrm{L}(\mathscr{H})$, where $\mathscr{H}$ is some Hilbert space. This is typical for systems of quantum mechanics, i. e. with finitely many degrees of freedom. Here we recover the familiar Hilbert space framework of standard quantum mechanics for systems with finitely many degrees of freedom by passing to the GNS representation.

Finally, we remark that to describe a system with pure quantum character, $\mathfrak{M}$ should be a factor, i. e. have a trivial center $Z(\mathfrak{M})=\mathfrak{M} \cap \mathfrak{M}^{\prime}=\mathbb{C} \mathbb{1}$ (like in the case of the example of a quantum particle in one dimension, where we have $\mathfrak{M}=\mathrm{L}(\mathscr{H})$ ). A nontrivial center of $\mathfrak{M}$ leads to the appearance of superselection rules which may be interpreted as classical properties of the system. We shall say more about this phenomenon in Chapter 3 .

\subsubsection{The Representation Problem}

The considerations of the last subsection lead us to the following procedure: One starts with an abstract $\mathrm{C}^{*}$-algebra $\mathfrak{A}$ which describes the basic structural features of the system. For example, a system with $n$ bosons in $d$ dimensions is described by the CCR algebra $\mathfrak{A}\left(\mathbb{C}^{n d}\right)$ with the canonical symplectic form $\sigma(f, g)=\operatorname{Im}\langle f, g\rangle, f, g \in \mathbb{C}^{n d}$ on $\mathbb{C}^{n d}$, considered as a real vector space. Then a state $\varphi$ on $\mathfrak{A}$, corresponding to a particular preparation procedure, leads by the GNS Theorem 1.2 to a Hilbert space representation of $\mathfrak{A}$ and to a von Neumann algebra $\mathfrak{M}$ which represent the physical observables of the system in state $\varphi$. The normal states in $\mathfrak{M}$ are called the statistical states of the system; they correspond to all states that can be obtained by operations (instruments) from $\mathfrak{M}$ applied to the state $\varphi$ and by forming convex combinations, i.e. statistical mixtures. Thus the vectors in the representation Hilbert space $\mathscr{H}_{\varphi}$ represent those states related to $\varphi$ by physically accessible operations, given by the self-adjoint operators in $\mathfrak{M}$. In this sense the GNS representation asso- 
ciated to $\varphi$ represents a "closed world" ${ }^{3}$, or a phase in thermodynamical terms.

Now the question arises how many of these exist. From the definition of the folium $N_{\varphi}$ of the state $\varphi$ in Appendix A it becomes clear that each folium corresponds to the set of states on $\mathfrak{A}$ which are obtainable from $\varphi$ by physical processes (i.e. instruments from $\mathfrak{A}$ and mixing), i.e. each folium (equivalently, each quasi-equivalence class of states) corresponds to the same "closed world". Thus we are asking for the quasi-equivalence classes of the algebra $\mathfrak{A}$. For bosonic systems with finitely many degrees of freedom there is only one folium if $\mathfrak{A}$ is the CCR algebra over a finitedimensional symplectic space as a consequence of the Stone-von Neumann Theorem A.16, and hence only one "closed world", which is (up to multplicity) the folium defined by the Fock representation. This corresponds to the Schrödinger representation of the canonical commutation relations, and we recover the framework of standard quantum mechanics.

The situation changes drastically if we consider systems with infinitely many degrees of freedom. Typically they arise in quantum field theory and statistical mechanics, where one considers systems in the thermodynamical limit. If the symplectic space $(\mathrm{S}, \sigma)$ is not finite-dimensional, there exist many different quasi-equivalence classes of representations of the CCR algebra $\mathfrak{A}(\mathrm{S}, \sigma)$, and the question arises which of these are physically meaningful and should be used in a particular situation. We shall not consider the problem to characterize physically relevant representations in quantum field theory and statistical mechanics in general, because this requires a number of notions, e. g. concerning the quasilocal structure of the algebras, which we want to avoid here. For an elementary introduction we refer to [29], and to [12] for a full discussion. But we shall explain some aspects concerning systems in thermal equilibrium with a heat reservoir.

We consider a system of bosons. The number of particles will not be fixed (grand canonical ensemble), thus we consider the Fock space $\mathscr{H}_{\mathrm{F}}=\bigoplus_{n=0}^{\infty} \mathscr{H}_{n}$, where $\mathscr{H}_{n}=\mathscr{H} \otimes_{\mathrm{s}} \cdots \otimes_{\mathrm{s}} \mathscr{H}$ is the Hilbert space of all symmetrized $n$-particle wave functions, and $\mathscr{H}$ is the one-particle Hilbert space. The relevant $\mathrm{C}^{*}$-algebra corresponding to this system is the CCR algebra $\mathfrak{A}(\mathscr{H})$, where $\mathscr{H}$ is considered as a symplectic space with canonical symplectic form. A concrete realization of $\mathfrak{A}(\mathscr{H})$ as a $\mathrm{C}^{*}$-algebra can be obtained from the Fock representation: Let $\omega_{\mathrm{F}}$ be the Fock state on $\mathfrak{A}(\mathscr{H})$, i. e. the state with generating functional $\phi_{\mathrm{F}}(f)=\mathrm{e}^{-\|f\|^{2} / 4}$ for all $f \in \mathscr{H}$. Consider the GNS-representation $\pi_{\mathrm{F}}$ associated to $\omega_{\mathrm{F}}$, realized on $\mathscr{H}_{\mathrm{F}}$ with cyclic vector $\xi_{\mathrm{F}} \in \mathscr{H}_{\mathrm{F}}$, where $\xi_{\mathrm{F}}=\left\{\xi_{n}\right\}_{n \in \mathbb{N} \cup\{0\}}$ such

${ }^{3}$ This formulation is borrowed from [29]. 
that $\xi_{0}=1$ and $\xi_{n}=0$ for $n>0$ (see e.g. [7] for details). Consider the Weyl system $W_{\mathrm{F}}(f)=\pi_{\mathrm{F}}(W(F)), f \in \mathrm{S}$, then according to Corollary A.6 the $\mathrm{C}^{*}$-algebra $\pi_{\mathrm{F}}(\mathfrak{A}(\mathscr{H}))=\varlimsup \operatorname{lin}\left\{W_{\mathrm{F}}(f): f \in \mathscr{H}\right\}$ is isomorphic to the abstract CCR algebra. We will use this concrete realization of the abstract CCR algebra below.

Let $V \subseteq \mathbb{R}^{d}$ be a bounded open region and suppose the bosons are confined to this region, i. e. we take the one particle Hilbert space $\mathscr{H}$ as $\mathscr{H}_{V}=\mathrm{L}^{2}(V)$. After choosing appropriate boundary conditions for $V$ the system has a Hamiltonian $H_{V}$ acting on $\mathscr{H}_{V}$, which is bounded below. For an inverse temperature $\beta>0$ the operator $\mathrm{e}^{-\beta H_{V}}$ will be of trace class (for reasonable interaction potentials of the particles) since $V$ is a bounded region. Let $\mathrm{d} \Gamma\left(H_{V}\right)$ be the second quantization of $H_{V}$, acting on $\mathscr{H}_{\mathrm{F}}$. Time evolution on $\pi_{\mathrm{F}}\left(\mathfrak{A}\left(\mathscr{H}_{V}\right)\right)$ is given by $\left\{\alpha_{t}^{V, \mu}\right\}_{t \in \mathbb{R}} \subseteq$ Aut $\pi_{\mathrm{F}}\left(\mathfrak{A}\left(\mathscr{H}_{V}\right)\right)$, where

$$
\alpha_{t}^{V, \mu}\left(W_{\mathrm{F}}(f)\right)=W_{\mathrm{F}}\left(\mathrm{e}^{\mathrm{i} t H_{V}}\right)=\mathrm{e}^{\mathrm{i} t \mathrm{~d} \Gamma\left(H_{V}-\mu N\right)} W_{\mathrm{F}}(f) \mathrm{e}^{-\mathrm{i} t \mathrm{~d} \Gamma\left(H_{V}-\mu N\right)},
$$

where $f \in \mathbf{S}$ and $t \in \mathbb{R}$; according to Proposition A.10 this defines a group of automorphisms on the CCR algebra. If $\mu \in \mathbb{R}$ is such that $\beta\left(H_{V}-\mu \mathbb{1}\right)>0$ the operator $\mathrm{e}^{-\beta \mathrm{d} \Gamma\left(H_{V}\right)+\beta \mu N}$ is trace class on $\mathscr{H}_{\mathrm{F}}$, see [7], Proposition 5.2.27. According to the principles of statistical mechanics the grand canonical equilibrium state of the system in equilibrium with a heat bath at inverse temperature $\beta$ and at chemical potential $\mu$ is given by the Gibbs state

$$
\omega_{\beta, \mu, V}(x)=\frac{\operatorname{tr}\left[x \mathrm{e}^{-\beta \mathrm{d} \Gamma\left(H_{V}\right)+\beta \mu N}\right]}{\operatorname{tr}\left[\mathrm{e}^{-\beta \mathrm{d} \Gamma\left(H_{V}\right)+\beta \mu N}\right]}, \quad x \in \mathrm{L}\left(\mathscr{H}_{\mathrm{F}}\right) .
$$

These states are not pure, hence the associated GNS representation is not irreducible. By isomorphy this defines define a state $\omega_{\beta, \mu, V}$ on the abstract CCR algebra $\mathfrak{A}\left(\mathscr{H}_{V}\right)$, and its generating functional is found to be

$$
\phi_{\beta, \mu, V}(f)=\exp \left(-\frac{1}{4}\left\langle f,\left(\mathbb{1}+z \mathrm{e}^{-\beta H_{V}}\right)\left(\mathbb{1}-z \mathrm{e}^{-\beta H_{V}}\right)^{-1} f\right\rangle\right), \quad f \in \mathscr{H},
$$

where $z=\mathrm{e}^{\beta \mu}$. Let us write now $\mathfrak{A}(V)=\mathfrak{A}\left(\mathscr{H}_{V}\right)$. For two open bounded regions $V_{1} \subseteq V_{2}$ we have the natural embedding $\mathfrak{A}\left(V_{1}\right) \subseteq \mathfrak{A}\left(V_{2}\right)$, hence we can consider the $\mathrm{C}^{*}$-inductive limit

$$
\mathfrak{A}_{\text {loc }}=\bigcup_{\substack{V \subseteq \mathbb{R}^{d} \\ \text { bded open region }}} \mathfrak{A}(V),
$$


called the local algebra. Its completion $\mathfrak{A}$ with respect to the norm topology is called the quasilocal algebra. The time evolution group has a natural extension $\left\{\alpha_{t}\right\}_{t \in \mathbb{R}} \subseteq$ Aut $\mathfrak{A}$ to $\mathfrak{A}$.

Having obtained a description of the finite systems which are confined to the region $V$, we want to perform the thermodynamical limit, i. e. we seek for a quantum mechanical description of the system if we take $V \uparrow \mathbb{R}^{d}$ while keeping the mean density

$$
\bar{\rho}=\omega_{\beta, \mu, V}(N) /|V|
$$

fixed, where $\omega_{\beta, \mu, V}(N)$ is the mean particle number in the state $\omega_{\beta, \mu, V}$. We first remark that the thermodynamical limit is not described by a Fock state. Suppose that the limiting state $\omega$ is Fock, then according to Theorem A.18 there exists a particle number operator $N_{\omega}$, which is a self-adjoint operator on $\mathscr{H}_{\omega}$, having a spectral measure $P$ and $\operatorname{spec} N_{\omega}=\{0,1,2, \ldots\}$. Now the probability for having at least $n$ particles in the system satisfies $\omega(P([n, \infty[)) \rightarrow 0$ as $n \rightarrow \infty$. This is clearly inconsistent with the concept of an infinitely extended system at nonzero density. Hence we are compelled to leave the realm of the Fock representation.

Let us now take the cubes $\left\{\left(x_{1}, \ldots, x_{d}\right) \in \mathbb{R}^{d}:\left|x_{i}\right|<L / 2, i=1, \ldots, d\right\}$ as the bounded open regions $V$ and let us consider the free case, i.e. we take as $H_{V}$ the self-adjoint extension of $-\Delta / 2 m$ to $\mathrm{L}^{2}(V)$ with periodic boundary conditions. The quasilocal algebra in this situation is taken to be $\mathfrak{A}_{\text {loc }}=\mathfrak{A}\left(\mathrm{C}_{\mathrm{c}}^{\infty}\left(\mathbb{R}^{d}\right)\right)$, where $\mathrm{C}_{\mathrm{c}}^{\infty}\left(\mathbb{R}^{d}\right)$ denotes the space of all continuous infinitely differentiable functions with compact support. Localization is obtained by restricting $f$ to functions with compact support, compare (1.21). In this case the thermodynamical limit at constant $\bar{\rho}$ of the states $\omega_{\beta, \bar{\rho}, V}$ in (1.19) was conjectured by Araki and Woods [38] to be given by the following generating functional:

$$
\begin{aligned}
& \phi_{\beta, \bar{\rho}}(f)=\mathrm{e}^{-\|f\|^{2} / 4} . \\
& \cdot\left\{\begin{array}{ll}
\exp \left(-\frac{1}{2(2 \pi)^{d}} \int_{\mathbb{R}^{d}} \frac{z_{\infty}}{\mathrm{e}^{\beta p^{2} / 2 m}-z_{\infty}}|\hat{f}(p)|^{2} \mathrm{~d} p\right) & \text { if } \bar{\rho} \leq \rho_{\mathrm{c}}(\beta) \\
\exp \left(-\frac{1}{2(2 \pi)^{d}} \int_{\mathbb{R}^{d}} \frac{1}{\beta \mathrm{e}^{p^{2} / 2 m}-1}|\hat{f}(p)|^{2} \mathrm{~d} p\right) \cdot & \text { if } \bar{\rho}>\rho_{\mathrm{c}}(\beta) \\
\cdot \exp \left(-\frac{1}{2}\left(\bar{\rho}-\rho_{\mathrm{c}}(\beta)\right)|\hat{f}(0)|^{2}\right) &
\end{array} .\right.
\end{aligned}
$$

Here $\rho_{\mathrm{c}}(\beta)=G(1, \beta)$ is the critical density for Bose-Einstein condensation, where

$$
G(z, \beta)=\frac{1}{(2 \pi)^{d}} \int_{\mathbb{R}^{d}} \frac{z}{\mathrm{e}^{\beta p^{2} / 2 m}-z} \mathrm{~d} p, \quad 0 \leq z \leq 1 .
$$


Moreover, $z_{\infty}$ is the unique solution of $\rho=G(z, \beta)$. We remark that below the critical density (the noncondensed regime) we can uniquely extend $\phi_{\beta, \bar{\rho}}$ and obtain the equilibrium state on $\mathfrak{A}\left(\mathrm{L}^{2}\left(\mathbb{R}^{d}\right)\right)$.

Concerning the thermodynamical limit we have the following result due to Lewis and Pulè [92].

Theorem 1.6 For any $\beta, \bar{\rho}>0$ and any test function $f \in \mathrm{C}_{\mathrm{c}}^{\infty}\left(\mathbb{R}^{d}\right)$ it follows that

$$
\lim _{L \rightarrow \infty} \phi_{\beta, \mu, V}(f)=\phi_{\beta, \bar{\rho}}(f),
$$

where $L$ is the edge length of the cube $V$.

The corresponding theorem for the canonical equilibrium states, whose proof is more involved, was given in [54]. See also [7]. The representation corresponding to the limiting state (the Araki-Woods representation) associated with $\phi_{\beta, \bar{\rho}}$ can be explicitly constructed, see [38, 92].

Another important property which the states $\omega_{\beta, \mu, V}$ satisfy is the KMS condition at value $\beta$ with respect to the automorphism group $\left\{\alpha_{t}^{V, \mu}\right\}_{t \in \mathbb{R}}$ (see Section 2.1.2 for some definitions and terminology concerning the KMS condition). In fact, it can be shown that, for bounded $V$, the KMS condition with respect to $\left\{\alpha_{t}^{V, \mu}\right\}_{t \in \mathbb{R}}$ characterizes the Gibbs state. The KMS condition has the property that it survives the thermodynamical limit, i. e. $\omega_{\beta, \bar{\rho}}$ corresponding to $\phi_{\beta, \bar{\rho}}$, is KMS as well. The KMS condition implies many properties of the GNS-representation $\pi_{\beta, \bar{\rho}}$ corresponding to the limiting state $[77,12]$; we quote the following result, which was first proved in [77].

Proposition 1.7 Let $\omega$ be a $\left\{\alpha_{t}\right\}_{t \in \mathbb{R}}-K M S$ state on a $C^{*}$-algebra $\mathfrak{A}$ at value $\beta$, let $\left(\pi_{\omega}, \xi_{\omega}, \mathscr{H}_{\omega}\right)$ be the corresponding GNS representation and $\mathfrak{M}=\pi_{\omega}(\mathfrak{A})^{\prime \prime}$. Let $\left\{u_{t}\right\}_{t \in \mathbb{R}}$ be the strongly continuous unitary group implementing $\left\{\alpha_{t}\right\}_{t \in \mathbb{R}}$, i.e. $\pi_{\omega}\left(\alpha_{t}(x)\right)=u_{t} \pi \omega(x) u_{t}^{*}, x \in \mathfrak{A}$. Then there exists a conjugation $J$ on $\mathscr{H}_{\omega}$, i.e. $J$ is antiunitary with $J^{2}=\mathbb{1}$, such that $\left[J, u_{t}\right]=0$ for all $t \in \mathbb{R}, J \xi_{\omega}=\xi_{\omega}$, and $J \mathfrak{M} J=\mathfrak{M}^{\prime}$.

This result reveals a close connection between KMS states and TomitaTakesaki modular theory, which is briefly summarized in Section 2.1.1. Indeed, a $\left\{\alpha_{t}\right\}_{t \in \mathbb{R}}$-KMS state at value $\beta$ is a faithful normal state on $\mathfrak{M}$ such that its modular group $\left\{\sigma_{t}^{\omega}\right\}_{t \in \mathbb{R}}$ satisfies $\alpha_{-\beta t}=\sigma_{t}^{\omega}$ for all $t \in \mathbb{R}$. Moreover, Proposition 1.7 establishes that equilibrium states are nonpure since the commutant $\pi_{\omega}(\mathfrak{A})^{\prime}$ is nontrivial, i. e. $\pi_{\omega}$ is not irreducible. 


\subsubsection{Infinite Systems}

We have already remarked that for quantum systems with a finite number of degrees of freedom we find that $\mathfrak{M}=\mathrm{L}(\mathscr{H})$. This is no longer the case if we consider systems with an infinite number of degrees of freedom. The differences are quite profound and have many physically relevant features.

We begin by very briefly recalling some features of the Murray-von Neumann classification of von Neumann algebras. This proceeds by studying the lattice of projections $\mathscr{P}(\mathfrak{M})$ of a von Neumann algebra. For details we refer to the literature on von Neumann algebras $[4,23,24,30]$. Two projections $p, q \in \mathscr{P}(\mathfrak{M})$ are called equivalent, in symbols $p \sim q$, if there exists a partial isometry $u \in \mathfrak{M}$ such that $p=u^{*} u$ and $q=u u^{*}$. We define $p \precsim q$ if $p \sim q^{\prime} \leq q$ for some $q^{\prime} \in \mathscr{P}(\mathfrak{M})$. The important comparison theorem states that for any two $p, q \in \mathscr{P}(\mathfrak{M})$ there exists a central projection $z \in \mathscr{P}(\mathfrak{M}) \cap Z(\mathfrak{M})$ such that $p z \precsim q z$ as well as $q z^{\perp} \precsim p^{\perp}$. In particular, if $\mathfrak{M}$ is a factor, either $p \precsim q$ or $q \precsim p$, hence in this case $\precsim$ totally orders $\mathscr{P}(\mathfrak{M})$. A projection $p$ in $\mathfrak{M}$ is called abelian if $p \mathfrak{M} p$ is abelian, finite if $p \sim q \leq p$ implies $p=q$, semifinite if every nonzero subprojection of $p$ contains a nonzero finite subprojection, infinite if $p$ is not finite, and purely infinite if it does not contain any nonzero finite subprojection. A von Neumann algebra is called finite, semifinite, infinite or purely infinite if $\mathbb{1}$ has this property. A von Neumann algebra $\mathfrak{M}$ is said to be of type $\mathrm{I}$ or discrete if $\mathfrak{M}=\{0\}$ or if every nonzero central projection contains an abelian projection (or equivalently that each projection majorizes some abelian projection). A von Neumann algebra is said to be of type III if is purely infinite. Finally, a von Neumann algebra is said to be of type II if it is semifinite and does not contain any abelian projections. If $\mathfrak{M}$ is of type II and finite it is said to be of type $\mathrm{II}_{1}$, else of type $\mathrm{II}_{\infty}$. The content of the following theorem is that the classification in types I, II and III is exhaustive.

Theorem 1.8 Any von Neumann algebra can be uniquely written as the direct sum of four von Neumann algebras of type $\mathrm{I}_{1} \mathrm{II}_{1}, \mathrm{II}_{\infty}$, III,

$$
\mathfrak{M}=\mathfrak{M}_{\mathrm{I}} \oplus \mathfrak{M}_{\mathrm{II}_{1}} \oplus \mathfrak{M}_{\mathrm{II}_{\infty}} \oplus \mathfrak{M}_{\mathrm{III}}
$$

If $\mathfrak{M}$ is a factor then $\mathfrak{M}$ is either of type $\mathrm{I}_{1} \mathrm{II}_{1}, \mathrm{II}_{\infty}$, or III.

The last statement follows since the von Neumann algebras on the right hand side of (1.25) are given by central projections. Clearly, every commutative von Neumann algebra is of type I and it can be shown that a factor of type $\mathrm{I}$ is always isomorphic to $\mathrm{L}(\mathscr{H})$ for some Hilbert space $\mathscr{H}$. The type II and type III algebras are more difficult to describe, see e. g. [4, 24]. 
Thus for quantum systems with finitely many degrees of freedom we always deal with factors of type I. But also commutative algebras may appear: When we consider a maximal system of compatible observables $S=\left\{x_{i}: i \in I\right\}$, where $I$ is some index set, we can form the commutative algebra $\mathfrak{M}_{S}$ generated by $S$. Maximality of $S$ is embodied in the property that $\mathfrak{M}_{S}$ is a maximal abelian subalgebra of $\mathfrak{M}$.

If we deal with systems which contain infinitely many degrees of freedom we leave the type I realm. One can argue that real systems are always finite and we can control experimentally only a finite number of degrees of freedom due to the limited resolution of our instruments, however, as in many other areas of physics, the generalization to infinitely many degrees of freedom is important since only this reveals the essential features of the physical situation.

We take up the discussion of the free Bose gas in the thermodynamical limit again. Here we find that at finite temperatures the von Neumann algebra $\mathfrak{M}$ is of type III. Let $K_{\beta}$ be the set of all $\left\{\alpha_{t}\right\}_{t \in \mathbb{R}}$-KMS states at value $\beta$ on a $C^{*}$-algebra $\mathfrak{A}$. For $\omega \in K_{\beta}$ let $\hat{\omega}$ be the extension of $\omega$ to a normal state on $\mathfrak{M}=\pi_{\omega}(\mathfrak{A})^{\prime \prime}$ and let $\pi_{\omega}\left(\alpha_{t}(x)\right)=u_{t} \pi_{\omega}(x) u_{t}^{*}=\hat{\alpha}_{t}\left(\pi_{\omega}(x)\right)$ for all $x \in \mathfrak{A}$. Then we have the following result, which is close to that in [81]; here we give a proof along different lines.

Proposition 1.9 Suppose that $\mathfrak{M}$ acts on a separable Hilbert space $\mathscr{H}$. Let $\omega \in K_{\beta}$ and $\beta>0$, write $\mathfrak{M}=\pi_{\omega}(\mathfrak{A})^{\prime \prime}$. If $\hat{\omega}$ is the only state invariant under $\left\{\hat{\alpha}_{t}\right\}_{t \in \mathbb{R}}$ then $\mathfrak{M}$ is a factor of type III.

Proof. Since every state in $K_{\beta}$ is invariant the assumption means that $\omega$ is extremal in $K_{\beta}$. Then we know that $\mathfrak{M}$ is a factor [7, 12]. If $a^{\prime} \in$ $\left(\mathfrak{M} \cup\left\{u_{t}\right\}_{t \in \mathbb{R}}\right)^{\prime}$ with $a^{\prime} \geq 0$ define $\hat{\omega}_{1}(x)=\hat{\omega}\left(x a^{\prime}\right)$ and suppose without loss of generality that $\hat{\omega}_{1}$ is a state. Then since $\hat{\omega}_{1}$ is invariant under $\left\{\alpha_{t}\right\}_{t \in \mathbb{R}}$ it follows that $\hat{\omega}=\hat{\omega}_{1}$. Define $a=J a^{\prime} J \in \mathfrak{M}$, then $a \xi_{\omega}=a^{\prime} \xi_{\omega}$ and $\hat{\omega}_{1}(x)=\hat{\omega}(x a)=\hat{\omega}\left(x a^{\prime}\right)$ for all $x \in \mathfrak{M}$. Now $\omega_{1}$ satisfies the KMS condition in the form $\hat{\omega}_{1}(x y)=\omega\left(\hat{\alpha}_{\mathrm{i} \beta}(y) x\right)$ for all $x, y \in \mathfrak{M}$. Then by the following calculation (cf. [12], p. 213) we have

$$
\begin{aligned}
\hat{\omega}(\text { yaxz }) & =\hat{\omega}\left(\hat{\alpha}_{-\mathrm{i} \beta}(x z) y a\right)=\hat{\omega}_{1}\left(\hat{\alpha}_{-\mathrm{i} \beta}(x z) y\right) \\
& =\hat{\omega}_{1}\left(\hat{\alpha}_{-\mathrm{i} \beta}(z) y x\right)=\hat{\omega}\left(\hat{\alpha}_{-\mathrm{i} \beta}(z) y x a\right) \\
& =\hat{\omega}(y x a z)
\end{aligned}
$$

for all $x, y, z \in \mathfrak{M}$, hence $[a, x]=0$ for any $x \in \mathfrak{M}$, hence $a \in \mathfrak{M} \cap \mathfrak{M}^{\prime}=\mathbb{C} \mathbb{1}$. We conclude that $\left(\mathfrak{M} \cup\left\{u_{t}\right\}_{t \in \mathbb{R}}\right)^{\prime}=\mathbb{C} \mathbb{1}$, hence $\left\{u_{t}\right\}_{t \in \mathbb{R}} \nsubseteq \mathfrak{M}$ in view of $\mathfrak{M}^{\prime}=J \mathfrak{M} J$, i. e. $\left\{\sigma_{t}^{\omega}\right\}_{t \in \mathbb{R}}$ not inner, hence for the Connes invariant 
$\mathrm{T}(\mathfrak{M}) \neq \mathbb{R}$. Since $\mathscr{H}$ is separable, $\mathfrak{M}_{*}$ is separable, thus $\mathrm{T}(\mathfrak{M}) \neq \mathbb{R}$ is equivalent to $\mathfrak{M}$ not being semifinite (see [4], III.4.6.6.), and we conclude. $\square$

We note that extremality of a state in $K_{\beta}$ means that it corresponds to a pure thermodynamical phase. It can be shown that $K_{\beta}$ is a simplex and that states in $K_{\beta}$ are extremal if and only if they are primary. Since equilibrium states are the KMS states (see [7] and also [27] for a discussion of this point) this result shows that we have phase coexistence.

According to the previous proposition, the von Neumann algebra $\mathfrak{M}$ corresponding to the free Bose gas in the thermodynamical limit in a primary state (pure thermodynamical phase) is a type III factor. This situation is typical for systems with infinitely many degrees of freedom: The local algebras in a local relativistic quantum field algebra, under certain additional conditions, have also been shown to be type III, see e. g. [69, 12].

The type III property entails certain differences which figure also in the discussion of foundational questions of quantum theory. For example, every projection in a type III algebra is infinite-dimensional, hence every eigenvalue of an observable in infinitely degenerate. More surprisingly, there are no pure vector states on a type II or type III factor $\mathfrak{M}$ :

Proposition 1.10 Let $\mathfrak{A}$ be a $C^{*}$-algebra and $\varphi$ a state on $\mathfrak{A}$. Assume that $\varphi$ is primary, i.e. that $\mathfrak{M}=\pi_{\varphi}(\mathfrak{A})^{\prime \prime}$ a factor. Then $\mathfrak{M}$ is of type I if and only if there are pure vector states on $\mathfrak{M}$.

For a proof see [10]. This shows that the popular calculations using vector states and observables with discrete spectrum and nondegenerate eigenvalues, which are widespread in the literature on the foundations of quantum physics, are restricted to the type I realm, hence they exclude infinite systems and do not stand up to their claimed generality.

\subsubsection{Differences Between Classical and Quantum Systems}

We have seen that the algebraic approach allows classical and quantum physics to be treated within one mathematical framework. The crucial difference between classical and quantum situations is the noncommutativity of the algebra of observables. We will explain here (without going into minute detail) which major physically relevant differences noncommutativity entails.

The first is a direct consequence of Lemma 1.5. If $\mathfrak{A}$ is noncommutative there are observables, say $x$, which have nonzero dispersion $\Delta_{\varphi}(x)$ for any state $\varphi$, so there are no dispersion free states. In contrast, as we have 
explained, pure states on a commutative $\mathrm{C}^{*}$-algebra are always dispersion free for any observable.

Consider a von Neumann algebra $\mathfrak{M}$ (acting on a separable Hilbert space) describing a physical system, together with a normal state $\varphi$. Any (maximal abelian) subalgebra $\mathfrak{N}$ of $\mathfrak{M}$ defines a classical probability model as we have seen in Subsection 1.2.1. This situation is described in more mathematical terms as follows: it can be shown that the subalgebra $\mathfrak{N}$ is generated by a single self-adjoint element $a \in \mathfrak{M}$, i. e. $\mathfrak{N}=\{a\}^{\prime \prime}$, or alternatively $\mathfrak{N}$ is obtained by taking all bounded Borel functions $f(a)=$ $\int_{\text {spec } a} f(\lambda) \mathrm{d} P(\lambda)$ of $a[30]$, here $P$ is the spectral measure of $a$. The classical probability space then is $\left(\mathbb{R}, \mathscr{B}^{1}, \varphi(P(\cdot))\right)$ and for each bounded Borel function $f: \mathbb{R} \longrightarrow \mathbb{R}$ (i. e. each observable in $\mathfrak{N}$ ) the expectation is $\varphi(f(a))=\int f(\lambda) \mathrm{d} \varphi(P(\lambda))$. Summarizing, quantum theory predicts a classical probability distribution for the observables in $\mathfrak{N}$, although $\mathfrak{M}$ need not be commutative. If we consider another abelian subalgebra $\mathfrak{N}_{0} \subseteq \mathfrak{M}$ different from $\mathfrak{N}$ then the classical probability model need not be the same as that assigned to $\mathfrak{N}$. In general, each maximal abelian subalgebra comes with its own classical probability space. Noncommuting observables cannot be represented on the same classical probability space as marginals of a single distribution. However, if an observable is a member of two different maximal abelian subalgebras, it produces the same distribution, although it is represented in each case by different random variables on different sample spaces. In modern terminology this dependence of the classical sample space on the (maximal) abelian subalgebra that is selected is called contextuality. For this reason it is wrong to say that upon making a measurement all observables assume values simultaneously, this is only true for the abelian subalgebra selected by the measurement.

At this point the question arises if it is really necessary to use noncommutative algebras in quantum physics at all. One might ask if it is possible to find a classical probability model that exactly reproduces the probability distribution for any observable of the system in any state, hence leading to a commutative algebra. Then contextuality would be an artifact of the mathematical model we have chosen but not a real phenomenon observable in the laboratory. Such an attempt is commonly discussed under the heading "hidden variables". Hence we look for a direct experimental test of noncommutativity of the algebra of observables. Such an experimentally testable criterion is provided by Bell's inequality. One version of a Bell inequality may be formulated as follows [88]: Let $\mathfrak{A}$ be a $\mathrm{C}^{*}$-algebra and let $p, q, p^{\prime}, q^{\prime} \in \mathscr{P}(\mathfrak{A})$. Assume that $\left[p, p^{\prime}\right]=\left[p, q^{\prime}\right]=0$. Define the self-adjoint elements $a=2 p-\mathbb{1}, b=2 q-\mathbb{1}$ and $a^{\prime}=2 p^{\prime}-\mathbb{1}, b^{\prime}=2 q^{\prime}-\mathbb{1}$ 
which all take the values \pm 1 . Next define the observable

$$
c=a\left(a^{\prime}+b^{\prime}\right)+b\left(b^{\prime}-a^{\prime}\right),
$$

we then have

$$
c^{2}=4+[a, b]\left[a^{\prime}, b^{\prime}\right]=4+16[p, q]\left[p^{\prime}, q^{\prime}\right] .
$$

If the observables $p, q, p^{\prime}, q^{\prime}$ are described by a classical probability distribution (which is, in view of Proposition 1.3, equivalent to the statement that they generate an abelian algebra), we find for the expectation of $c$ in any state $\varphi$ that

$$
|\varphi(c)| \leq 2,
$$

which is Bell's inequality. Now by the Hahn-Banach theorem for states we can find a state $\varphi$ on $\mathfrak{A}$ such that $\varphi\left([p, q]\left[p^{\prime}, q^{\prime}\right]\right)=\left\|[p, q]\left[p^{\prime}, q^{\prime}\right]\right\|$ (possibly by interchanging the roles of $p$ and $q)$. Then $\varphi\left(c^{2}\right)=4+16\left\|[p, q]\left[p^{\prime}, q^{\prime}\right]\right\| \leq$ $\left\|c^{2}\right\|$, which implies $\left\|c^{2}\right\|=\|c\|^{2}=4+16\left\|[p, q]\left[p^{\prime}, q^{\prime}\right]\right\|$. We see, again by the Hahn-Banach theorem for states, that we can find a state $\psi$ on $\mathfrak{A}$ such that

$$
|\psi(c)|=\sqrt{4+16\left\|[p, q]\left[p^{\prime}, q^{\prime}\right]\right\|},
$$

which violates (1.26) if the projections $p, q$ and $p^{\prime}, q^{\prime}$ are not compatible. Thus in a system with quantum character Bell's inequality is violated for suitably chosen observables and state $\psi$. If we can experimentally verify the violation of Bell's inequality (1.26) then the conclusion is that there exists no underlying classical probabilistic model whatsoever. We stress that the merit of Bell's inequality lies in its experimental testability which is usually realized as a correlation measurement between four observables $a, b$ and $a^{\prime}, b^{\prime}$ in an entangled state where $a, b$ and $a^{\prime}, b^{\prime}$ are measured in spacelike separated locations [41] to exclude causal influences between the two experimental setups which might be held responsible for the observed correlations. Notice however that no assumptions about locality (except for the choice of $p, q$ and $p^{\prime}, q^{\prime}$, which must be "local", cf. [59], or, in better terminology, noncontextual in the sense that the random variables representing $p^{\prime}$ and $q^{\prime}$ do not depend on whether $p$ or $q$ is being measured) and causality were necessary in the above argument. Finally we remark that above $\mathfrak{A}$ can be replaced by a von Neumann algebra and the states can be taken as normal.

Another phenomenon arising in the noncommutative realm is entanglement. A subsystem of a larger system is described by a subalgebra of the algebra of observables of the larger system. Thus if a system is considered 
to be composed of two parts with von Neumann algebras $\mathfrak{M}$ and $\mathfrak{N}$ acting on the same Hilbert space $\mathscr{H}$, the von Neumann algebra of the total system is taken to be $\mathfrak{M} \vee \mathfrak{N}=(\mathfrak{M} \cup \mathfrak{N})^{\prime \prime}$. In many cases, the algebra of the total system has the form $\mathfrak{M} \bar{\otimes} \mathfrak{N} \subseteq \mathrm{L}\left(\mathscr{H}_{1} \otimes \mathscr{H}_{2}\right)$, the von Neumann tensor product of $\mathfrak{M}$ and $\mathfrak{N}$, and $\mathfrak{M}$ is identified with $\mathfrak{M} \otimes \mathbb{1}_{\mathscr{H}_{2}}$ and $\mathfrak{N}$ with $\mathbb{1}_{\mathscr{H}_{1}} \otimes \mathfrak{N}$; then $\mathfrak{M} \otimes \mathfrak{N}=(\mathfrak{M} \odot \mathfrak{N})^{\prime \prime} \cong \mathfrak{M} \vee \mathfrak{N}$, with $\odot$ the algebraic tensor product. A state $\varphi$ on $\mathfrak{M} \vee \mathfrak{N}$ is called a product state if it satisfies $\varphi(x y)=\varphi(x) \varphi(y)$ for any $x \in \mathfrak{M}$ and $y \in \mathfrak{N}$. The states in the closed convex hull (either in norm topology or $\sigma\left((\mathfrak{M} \vee \mathfrak{N})_{*}, \mathfrak{M} \vee \mathfrak{N}\right)$-topology) of the set of all product states, i. e. mixtures of separable states, are called nonentangled or separable. If a state is not separable it is called entangled. The next is Raggio's theorem [111].

Theorem 1.11 Let $\mathfrak{M}$ and $\mathfrak{N}$ be two von Neumann algebras. Then the following assertions are equivalent:

1. Consider the observable $c=a \otimes\left(a^{\prime}+b^{\prime}\right)+b \otimes\left(b^{\prime}-a^{\prime}\right)$ in $\mathfrak{M} \bar{\otimes} \mathfrak{N}$, where $a, b \in$ ball $\mathfrak{M}^{\mathrm{sa}}$ and $a^{\prime}, b^{\prime} \in$ ball $\mathfrak{N}^{\mathrm{sa}}$. Then Bell's inequality $|\varphi(c)| \leq 2$ holds for any normal state $\varphi$.

2. $\mathfrak{M}$ or $\mathfrak{N}$ is abelian.

3. Every normal state on $\mathfrak{M} \bar{\otimes} \mathfrak{N}$ is separable.

Hence in a composite system in which both parts are of quantum character and hence are described by nonabelian algebras the phenomenon of entanglement arises. Moreover, this theorem makes clear that there is a close connection between Bell's inequality and entanglement. In fact, it can be shown that for a separable state Bell's inequality always holds [42].

The state space has different geometrical properties depending on the algebra being abelian or not. Recall that the set of pure states on a $\mathrm{C}^{*}$ algebra $\mathfrak{A}$ are exactly the extreme points of $\mathscr{S}(\mathfrak{A})$. It can be shown that $\varphi \in \mathscr{S}(\mathfrak{A})$ is pure if and only if the GNS representation $\pi_{\varphi}: \mathfrak{A} \longrightarrow \mathrm{L}\left(\mathscr{H}_{\varphi}\right)$ is irreducible, which is in turn equivalent to $\mathfrak{M}=\pi_{\varphi}(\mathfrak{A})^{\prime \prime}=\mathrm{L}\left(\mathscr{H}_{\varphi}\right)$. Generally, pure states are those which produce the least dispersion among all states. As a consequence of the Krein-Milman theorem, since $\mathscr{S}(\mathfrak{A})$ is $\sigma\left(\mathfrak{A}^{*}, \mathfrak{A}\right)$-compact, it is the weak* closed convex hull of the set of all pure states. Thus all states are obtained by mixing of pure states, and any state may be approximated by mixtures of pure states. But the convex hull of all pure states is not a simplex when $\mathfrak{A}$ is noncommutative: a given nonpure state can be written in many ways as a mixture of pure states. This cannot happen when $\mathfrak{A}$ is abelian. In fact $\mathfrak{A}$ is abelian if and only if $\mathscr{S}(\mathfrak{A})$ is a simplex. 


\subsection{Open Systems and Dynamics}

We have argued that, under very general assumptions, a (quantum or classical) system is described by a von Neumann algebra $\mathfrak{M}$ acting on a Hilbert space $\mathscr{H}$. Then a subsystem is described by a subclass of observables of $\mathfrak{M}$ pertaining to the subsystem only, for practical reasons we can always consider the von Neumann subalgebra $\mathfrak{N} \subseteq \mathfrak{M}$ generated by these observables. In specific situations we can say more about the inclusion $\mathfrak{N} \subseteq \mathfrak{M}$.

\subsubsection{Dynamics of Subsystems and Conditional Expectations}

Let $\mathfrak{A}$ and $\mathfrak{B}$ be two $\mathrm{C}^{*}$-algebras. Recall that a bounded map $T: \mathfrak{A} \longrightarrow \mathfrak{B}$ is called positive if $T\left(\mathfrak{A}^{+}\right) \subseteq \mathfrak{B}^{+}$, strongly positive if it satisfies Kadison's inequality,

$$
T(x)^{*} T(x) \leq\|T\| T\left(x^{*} x\right), \quad x \in \mathfrak{A},
$$

$n$-positive $(n \in \mathbb{N})$ if the induced map $T_{n}: M_{n}(\mathfrak{A}) \longrightarrow M_{n}(\mathfrak{B})$, defined by $T_{n}\left(\left[x_{i j}\right]\right)=\left[T\left(x_{i j}\right)\right]$ for $\left[x_{i j}\right] \in M_{n}(\mathfrak{A})$, is positive, and completely positive if $T$ is $n$-positive for any $n \in \mathbb{N}$. It is known that 2-positivity implies strong positivity but not conversely [56]. If $\mathfrak{A}$ or $\mathfrak{B}$ is abelian then all above positivity concepts coincide [30], moreover, $n$-positivity is equivalent to the condition

$$
\sum_{i, j=1}^{n} y_{i}^{*} T\left(x_{i}^{*} x_{j}\right) y_{j} \geq 0
$$

for all $x_{1}, \ldots, x_{n} \in \mathfrak{A}$ and $y_{1}, \ldots, y_{n} \in \mathfrak{B}$. We will frequently use the following fact: suppose that the linear map $T: \mathfrak{A} \longrightarrow \mathfrak{B}$ is unital (i.e. $T(\mathbb{1})=\mathbb{1}$ ). Then $T$ is positive if and only if it is a contraction (i.e. $\|T\| \leq 1)$.

We begin by defining conditional expectations on $\mathrm{C}^{*}$-algebras and explain their connection to open systems.

Definition 1.12 Let $\mathfrak{A}$ be a $C^{*}$-algebra and $\mathfrak{B} \subseteq \mathfrak{A}$ a $C^{*}$-subalgebra. $A$ linear map $E: \mathfrak{A} \longrightarrow \mathfrak{B}$ is called a conditional expectation if it is a completely positive contraction and satisfies $E(x y)=x E(y)$ and $E(x y)=$ $E(x) y$ (B-linearity) for all $x \in \mathfrak{A}$ and $y \in \mathfrak{B}$.

The following characterization of conditional expectations [127, 4] (Tomiyama's theorem) is frequently useful. 
Theorem 1.13 Let $\mathfrak{A}$ be a $C^{*}$-algebra, $\mathfrak{B} \subseteq \mathfrak{A}$ a $C^{*}$-subalgebra, and let $E: \mathfrak{A} \longrightarrow \mathbb{B}$ be a linear map. Then $E$ is a conditional expectation if and only if $E \circ E=E$ and $\|E\|=1$. Moreover, $E$ is a conditional expectation if and only if $E \circ E=E$ and $E$ is a positive $\mathfrak{B}$-linear map.

Let us consider two von Neumann algebras $\mathfrak{M}_{1} \subseteq \mathrm{L}\left(\mathscr{H}_{1}\right)$ and $\mathfrak{M}_{2} \subseteq$ $\mathrm{L}\left(\mathscr{H}_{2}\right)$ describing two separate independent physical systems, then the joint system has the algebra $\mathfrak{N}=\mathfrak{M}_{1} \bar{\otimes} \mathfrak{M}_{2}$, acting on $\mathscr{H}=\mathscr{H}_{1} \otimes \mathscr{H}_{2}$. Recall that the predual of $\mathrm{L}(\mathscr{H})$ is $\mathrm{T}(\mathscr{H})$, the space of all trace class operators on $\mathscr{H}$, and any normal state $\varphi$ can be represented by a density matrix $\rho \in \mathrm{T}(\mathscr{H})$ with $\rho \geq 0$ and $\operatorname{tr} \rho=1$, such that $\varphi(x)=\operatorname{tr}(\rho x)$ for all $x \in \mathrm{L}(\mathscr{H})$. The following construction [24] of conditional expectations will turn out to be useful in this situation.

Proposition 1.14 Let $\varphi_{0}$ be a normal state on $\mathfrak{M}_{2}$. Then there exists a normal conditional expectation $E_{\varphi_{0}}: \mathfrak{N} \longrightarrow \mathfrak{M}_{1} \otimes \mathbb{1}$ such that

$$
\left(\varphi \otimes \varphi_{0}\right)(x)=\left(\varphi \otimes \varphi_{0}\right)\left(E_{\varphi_{0}}(x)\right), \quad x \in \mathfrak{M}
$$

for any normal state $\varphi$ on $\mathfrak{M}_{1}$.

Proof. Fix $x \in \mathfrak{N}$ and define $m(x, \varphi)=\left(\varphi \otimes \varphi_{0}\right)(x)$, then $\|m(x, \varphi)\| \leq$ $\|\varphi\| \cdot\left\|\varphi_{0}\right\| \cdot\|x\|$, so $\varphi \mapsto m(x, \varphi)$ is in $\left(\mathfrak{M}_{1, *}\right)^{*}=\mathfrak{M}_{1}$, i. e. there exists a unique $\Phi_{\varphi_{0}}(x) \in \mathfrak{M}_{1}$ such that $\left(\varphi \otimes \varphi_{0}\right)(x)=\varphi\left(\Phi_{\varphi_{0}}(x)\right)$. If we define $E_{\varphi_{0}}(x)=\Phi_{\varphi_{0}}(x) \otimes \mathbb{1}$ we get a map $E$ with the property (1.30). Since $\left\|\Phi_{\varphi_{0}}(x)\right\| \leq\|x\|$ and $\Phi_{\varphi_{0}}(\mathbb{1})=\mathbb{1}$ we get $\left\|E_{\varphi_{0}}\right\|=1$, hence $E_{\varphi_{0}}$ is positive and a conditional expectation by Tomiyama's theorem. The map $\mathfrak{N} \ni x \mapsto\left\langle x, \varphi \otimes \varphi_{0}\right\rangle=\left\langle\Phi_{\varphi_{0}}(x), \varphi\right\rangle$ is normal, hence $x \mapsto \Phi_{\varphi_{0}}(x)$ is $\sigma\left(\mathfrak{N}, \mathfrak{N}_{*}\right)-\sigma\left(\mathfrak{M}_{1}, \mathfrak{M}_{1, *}\right)$-continuous, and we conclude that $E_{\varphi_{0}}$ is normal. $\square$

If $\mathfrak{N}=\mathfrak{M}_{1} \bar{\otimes} \mathfrak{M}_{2}$ as above suppose that the system is in the statistical state described by the density matrix $\rho \in \mathrm{T}(\mathscr{H})$. Then the state as witnessed by an observer whose observations are confined to $\mathfrak{M}_{1}$ is given by the

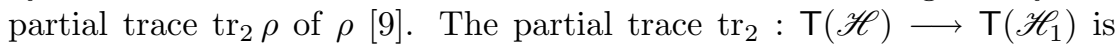
defined by

$$
\operatorname{tr}\left[\left(\operatorname{tr}_{2} \rho\right) x\right]=\operatorname{tr}[(x \otimes \mathbb{1}) \rho], \quad x \in \mathrm{L}\left(\mathscr{H}_{1}\right) .
$$

Then $\operatorname{tr}_{2}$ maps density matrices in $\mathrm{T}(\mathscr{H})$ to density matrices in $\mathrm{T}\left(\mathscr{H}{ }_{1}\right)$. Suppose that the time evolution of the total system is given by a Hamiltonian $H$ on $\mathscr{H}_{1} \otimes \mathscr{H}_{2}$. In many applications $H$ splits up into three parts,

$$
H=H_{1} \otimes \mathbb{1}+\mathbb{1} \otimes H_{2}+g H_{\mathrm{I}},
$$

where $H_{1}$ and $H_{2}$ describe the free evolution of systems 1 and 2, respectively, and $H_{\mathrm{I}}$ describes the interaction between the two systems; 
$g \in \mathbb{R}$ is a coupling constant. Then the Schrödinger picture time evolution on the density matrices of the total system is implemented by $H$, i. e. $\rho(t)=\mathrm{e}^{-\mathrm{i} t H} \rho \mathrm{e}^{\mathrm{i} t H}$ for any $t \in \mathbb{R}$ and any initial density matrix $\rho$. The time evolution of the reduced system is given by taking the partial trace:

$$
T_{*, t}(\varphi):=\rho_{1}(t)=\operatorname{tr}_{2}\left(\mathrm{e}^{-\mathrm{i} t H} \rho \mathrm{e}^{\mathrm{i} t H}\right),
$$

where $\varphi\left(x_{1}\right)=\operatorname{tr}\left(\rho x_{1}\right)$ for any $x_{1} \in \mathfrak{M}_{1}$ is the corresponding normal state. Thus an observer confined to system 1 would witness the state $\rho_{1}(t) \in \mathrm{T}\left(\mathscr{H}_{1}\right)$ at time $t$. We wish to consider this situation in the algebraic framework by using von Neumann algebra concepts. To achieve this we additionally assume that initially (i. e. at $t=0$ ) the density matrix of the total system is given by

$$
\rho(0)=\rho \otimes \rho_{0},
$$

where $\rho \in \mathrm{T}\left(\mathscr{H}_{1}\right)$ and $\rho_{0} \in \mathrm{T}\left(\mathscr{H}_{2}\right)$. Thus initially the state of the total system is in product form, i. e. nonentangled. We write $\varphi_{0}\left(x_{2}\right)=\operatorname{tr}\left(\rho_{0} x_{2}\right)$, where $x_{2} \in \mathfrak{M}_{2}$, for the corresponding normal state on $\mathfrak{M}_{2}$. Then by duality and Proposition 1.14 we find

$$
\begin{aligned}
\left\langle x_{1}, T_{t, *}(\varphi)\right\rangle & =\operatorname{tr}\left[x_{1} \operatorname{tr}_{2}\left(e^{-\mathrm{i} t H} \rho \otimes \rho_{0}\right) \mathrm{e}^{\mathrm{i} t H}\right] \\
& =\operatorname{tr}\left[\left(x_{1} \otimes \mathbb{1}\right) \mathrm{e}^{-\mathrm{i} t H}\left(\rho \otimes \rho_{0}\right) \mathrm{e}^{\mathrm{i} t H}\right] \\
& =\operatorname{tr}\left[\mathrm{e}^{\mathrm{i} t H}\left(x_{1} \otimes \mathbb{1}\right) \mathrm{e}^{-\mathrm{i} t H}\left(\rho \otimes \rho_{0}\right)\right] \\
& =\operatorname{tr}\left[E_{\varphi_{0}}\left(\mathrm{e}^{\mathrm{i} t H}\left(x_{1} \otimes \mathbb{1}\right) \mathrm{e}^{-\mathrm{i} t H}\right) \rho\right] \\
& =\left\langle T_{t}\left(x_{1}\right), \varphi\right\rangle,
\end{aligned}
$$

where we have put

$$
T_{t}\left(x_{1}\right)=E_{\varphi_{0}}\left(\alpha_{t}\left(x_{1} \otimes \mathbb{1}\right)\right), \quad x_{1} \in \mathfrak{M}_{1}, t \geq 0
$$

and $\alpha_{t}(x)=\mathrm{e}^{\mathrm{i} t H} x \mathrm{e}^{-\mathrm{i} t H}$ for any $x \in \mathfrak{N}$ and $t \in \mathbb{R}$. Then $\left\{\alpha_{t}\right\}_{t \in \mathbb{R}} \subseteq$ Aut $\mathfrak{N}$ is the automorphism group describing time evolution of the joint system in the Heisenberg picture. (Of course, starting with $\mathfrak{N}=\mathfrak{M}_{1} \bar{\otimes} \mathfrak{M}_{2}$ and a Hamiltonian $H$ one has to ensure that $\alpha_{t}$ leaves $\mathfrak{N}$ invariant. For example, this will be the case in systems when there is an energy observable $H$, then $H$ is affiliated with $\mathfrak{N}$, see the discussion in [6].) The family of maps $\left\{T_{t}\right\}_{t \geq 0}$ is called the reduced dynamics of the open system $\mathfrak{M}_{1}$. In general it has properties different from the dynamics of a closed system, which is given by a group of automorhisms $\left\{\alpha_{t}\right\}_{t \in \mathbb{R}}$. Most importantly it is, in general, irreversible, i. e. if we know $y=T_{t}(x)$ for some $t>0$ we cannot infer anything about the observable at earlier times because $T_{t}$, for 
$t>0$, need not be invertible, in contrast to an automorphism. Thus the reduced dynamics is able to describe phenomena such as irreversibility and approach to equilibrium - and, as we shall see in Chapter 3, also decoherence. See also the remarks in the next subsection.

\subsubsection{Reduced Dynamics and Dynamical Semigroups}

The situation encountered in the previous section can be generalized as follows. Consider a system described by a von Neumann algebra $\mathfrak{N}$ acting on a Hilbert space $\mathscr{H}$. This system is thought of as closed, hence its time evolution is described by an automorphism group $\left\{\alpha_{t}\right\}_{t \in \mathbb{R}}$. A natural continuity requirement (ultraweak continuity) is that $\mathbb{R} \ni t \mapsto\left\langle\alpha_{t}(x), \varphi\right\rangle$ be continuous for any $x \in \mathfrak{N}$ and $\varphi \in \mathfrak{N}_{*}$; then the expectation values of all observables in all statistical states move continuously with time. Consider a subsystem of this system, given by a von Neumann algebra $\mathfrak{M}$; the property of being a subsystem is described by the inclusion $i: \mathfrak{M} \longrightarrow$ $\mathfrak{N}$, which is a normal injective ${ }^{*}$-homomorphism. Suppose there exists a normal map $E: \mathfrak{N} \longrightarrow \mathfrak{M}$ such that $i \circ E$ is a (normal) conditional expectation onto $i(\mathfrak{M})$. Then the reduced dynamics of the subsystem is defined as the family of maps $\left\{T_{t}\right\}_{t \geq 0} \subseteq \mathrm{L}(\mathfrak{M})$, where

$$
T_{t}(x)=E \circ \alpha_{t} \circ i, \quad x \in \mathfrak{M}, t \geq 0 .
$$

This means the diagram

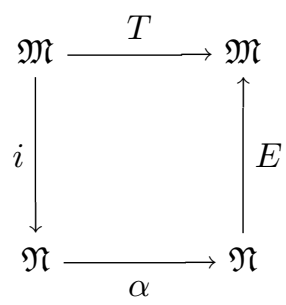

commutes. We can infer some properties of $\left\{T_{t}\right\}_{t \geq 0}$.

Proposition 1.15 In the above setting, the reduced dynamics $\left\{T_{t}\right\}_{t \geq 0}$ is a family of normal completely positive and unital maps, i. e. $T_{t}(\mathbb{1})=\mathbb{1}$ for all $t \geq 0$. For any $x \in \mathfrak{M}$ the map $\mathbb{R}^{+} \ni t \mapsto T_{t}(x)$ is ultraweakly continuous. Moreover, each $T_{t}$ is contractive, $\left\|T_{t}(x)\right\| \leq\|x\|$ for any $x \in \mathfrak{M}$.

The proof is obvious. Abstracting further we may call a family of normal completely positive unital (hence contractive) maps $\left\{T_{t}\right\}_{t \geq 0}$ on a von Neumann algebra a dissipative dynamics. 
The model for the above concept of reduced dynamics and the properties described in Proposition 1.15 was given in the previous subsection. There we have studied the special case $\mathfrak{N}=\mathfrak{M}_{1} \bar{\otimes} \mathfrak{M}_{2}$, with the inclusion given by $i\left(x_{1}\right)=x_{1} \otimes \mathbb{1}$ for all $x_{1} \in \mathfrak{M}_{1}$, and $\left\{T_{t}\right\}_{t \geq 0}$ arising from a conditional expectation with respect to a normal state $\varphi_{0}$ on $\mathfrak{M}_{2}$ by (1.33). The reason that we were able to construct conditional expectation $E_{\varphi_{0}}$ onto $i\left(\mathfrak{M}_{1}\right)$ was our assumption (1.32), i. e. that the system initially is in a product state. If we depart from this assumption the reduced dynamics on the algebra, constructed from the partial trace by duality, is not given by a conditional expectation and hence need not be completely positive. In the more general situation where the subalgebra $\mathfrak{M}$ is not a tensor factor of $\mathfrak{N}$ there is, in general, no normal conditional expectation onto $\mathfrak{M}$. In such situations we can still consider families of maps $\left\{T_{t}\right\}_{t \geq 0} \subseteq \mathrm{L}(\mathfrak{M})$ to phenomenologically describe the reduced dynamics, but with possibly weaker properties. For example, the justification of complete positivity is lost in this situation and may be reduced to positivity, similarly the unital property may be reduced to $T_{t}(\mathbb{1}) \leq \mathbb{1}$. However, positivity and normality as well as ultraweak continuity of $t \mapsto T_{t}(x)$ should be retained. There is an ongoing debate in the literature about the role of complete positivity, see the work of $\mathrm{F}$. Benatti et. al. [44, 45] and references therein. Finally, we remark that families of maps as in Proposition 1.15 can also arise as the solution of stochastic evolution equations, see the review [123] for more information on this topic.

The situation in which the reduced dynamics $\left\{T_{t}\right\}_{t \geq 0}$ of an open quantum system is memory free is of particular importance. By this we mean that, at any time $t_{0}>0$, the future time development at times $t_{0}+t$, depends only on $T_{t_{0}}(x)$, and not on the initial condition $T_{0}(x)=x$. This is modeled by requiring that $\left\{T_{t}\right\}_{t \geq 0}$ satisfies the semigroup property,

$$
\begin{gathered}
T_{s} \circ T_{t}=T_{s+t}, \quad s, t \geq 0, \\
T_{0}=\mathrm{id} .
\end{gathered}
$$

Time evolutions with this property are called Markovian. The semigroup property is a huge simplification, for it allows a description of the reduced dynamics by a single operator on $\mathfrak{M}$, the generator of the semigroup, and we have the whole machinery of the theory one-parameter semigroups $[6$, $11,32]$ available. In many physically interesting situations it is possible to approximate the reduced dynamics by a semigroup. E. B. Davies $[60,61$, 62 ] has studied Markovian approximations from a general point of view, see also the review [122] for information and references of the various limiting modes (weak coupling, singular coupling, low density limit, etc.). 
Examples of particular systems in which the existence of a Markovian limit can be rigorously proven are, for example, considered in [110, 78, 107]. This leads us to the following definition.

Definition 1.16 Let $\mathfrak{M}$ be a von Neumann algebra. A quantum dynamical semigroup is a family of normal completely positive and unital maps $\left\{T_{t}\right\}_{t \geq 0} \subseteq \mathrm{L}(\mathfrak{M})$ satisfying the semigroup property (1.35) and (1.35). Moreover, we require the map $\mathbb{R}^{+} \ni t \mapsto T_{t}(x)$ to be ultraweakly continuous for any $x \in \mathfrak{M}$.

Notice that then automatically $\left\|T_{t}\right\| \leq 1$ for any $t \geq 0$. For general information about quantum dynamical semigroups see e.g. [1, 9]. Quantum dynamical semigroups in the sense of this definition will be our basic model for the time evolution of an open system in the present work. However, many mathematical theorems will also hold for semigroups $\left\{T_{t}\right\}_{t \geq 0}$ (or mere families of operators) with weaker properties; sometimes we will indicate this added generality.

Quantum dynamical semigroups do not only arise as Markovian approximations of reduced dynamics but also in phenomenological descriptions of the influence of the environment on an open system. Often, a phenomenological prescription of a quantum dynamical semigroup is the starting point because it is usually difficult to rigorously perform a Markovian limit [123].

Quantum dynamical semigroups may be considered as a generalization of a classical Markov process. A time homogeneous stochastic process $\left\{X_{t}\right\}_{t \geq 0}$ (with values in $\mathbb{R}$ ) in continuous time can be described by the associated transition operators. Let $\mu_{s, t}\left(X_{s}, B\right)=P\left\{X_{t} \in B \mid X_{s}\right\}$, for $s \leq t$ and Borel sets $B$, be the corresponding transition kernels; by time homogeneity $\mu_{t, s}=\mu_{t-s}$. Then, say, on $\mathrm{C}(\mathbb{R})$ we can define the transition operators

$$
T_{t}(f)(x)=\int f(y) \mu_{t}(x, \mathrm{~d} y), \quad x \in \mathbb{R}, f \in \mathrm{C}(\mathbb{R}) .
$$

Each $T_{t}$ is a positive bounded operator, and $\left\{T_{t}\right\}_{t \geq 0}$ is a semigroup (i.e. satisfies (1.35)) if and only if

$$
\mu_{s} \mu_{t}(x, B)=\int \mu_{t}(y, B) \mu_{s}(x, \mathrm{~d} y)=\mu_{s, t}(x, B),
$$

i. e. if and only if $\left\{X_{t}\right\}_{t \geq 0}$ is a Markov process. In this sense (up to technicalities) a quantum dynamical semigroup is the noncommutative generalization of a Markov process. The continuity of $t \mapsto T_{t}(f)$ of course 
depends on the continuity properties of $\left\{X_{t}\right\}_{t \geq 0}$, see e.g. [16]. Suppose that $\nu$ is an invariant distribution of $\left\{\mu_{t}\right\}_{t \geq 0}$, i. e. $\nu \mu_{t}=\nu$ for any $t \geq 0$. Then (1.37) defines a contraction on $L^{1}(\mathbb{R}, \nu)$, and if it is strongly continuous (equivalently weakly continuous) and satisfies $T_{t}(1)=1$ for any $t \geq 0$ (so-called conservativity) we get by duality a quantum dynamical semigroup $\left\{T_{t}^{*}\right\}_{t \geq 0}$ on the dual $L^{\infty}(\mathbb{R}, \nu)$ of $L^{1}(\mathbb{R}, \nu)$. Moreover, the invariant measure $\nu$ defines a normal state on $L^{\infty}(\mathbb{R}, \nu)$ and we have $T_{t}^{*} \circ \nu=\nu$ for any $t \geq 0$. Thus the quantum dynamical semigroup $\left\{T_{t}^{*}\right\}_{t \geq 0}$ has a normal invariant state. We will consider normal invariant states for abstract quantum dynamical semigroups in the next section since they play an important role (both technically and physically) in many applications.

\subsection{Fixed Points and Invariant States}

This section collects some technical results of families of maps in $\mathrm{L}(\mathfrak{A})$ and $\mathrm{L}(\mathfrak{M})$ for a $\mathrm{C}^{*}$-algebra $\mathfrak{A}$ and a von Neumann algebra $\mathfrak{M}$ which will be needed in the sequel. In particular, we will apply them to quantum dynamical semigroups on von Neumann algebras.

\subsubsection{Fixed Points}

For any set $S$ of linear operators from a Banach space $\mathrm{X}$ into $\mathrm{X}$ we denote by fix $S$ the set of all fixed points of $S$, i. e. fix $S=\{x \in \mathrm{X}: T(x)=$ $x$ for all $T \in S\}$. The next proof uses a method of [66].

Lemma 1.17 Let $\mathfrak{A}$ be a $C^{*}$-algebra and $S \subseteq \mathrm{L}(\mathfrak{M})$ a family of strongly positive contractive linear maps on $\mathfrak{A}$. Suppose that $\Phi$ is a faithful family of subinvariant states for $S$, i.e. $\varphi(T(x)) \leq \varphi(x)$ for all $x \in \mathfrak{A}^{+}$and $\varphi \in \Phi$. Then fix $S$ is a $C^{*}$-subalgebra of $\mathfrak{A}$.

Proof. By positivity of $T \in S$ it follows that fix $S$ is *-invariant. Let $x \in$ fix $S$ and $T \in S$. Then $x^{*} x=T(x)^{*} T(x) \leq T\left(x^{*} x\right)$ and by invariance $\varphi\left(T\left(x^{*} x\right)\right) \leq \varphi\left(x^{*} x\right)$, hence $0 \leq \varphi\left(T\left(x^{*} x\right)-x^{*} x\right) \leq 0$ for any $\varphi \in \Phi$, hence $T\left(x^{*} x\right)=x^{*} x$. Now define for each $T \in S$ and state $\varphi \in \Phi$ the sesquilinear form $D_{T, \varphi}: \mathfrak{A} \times \mathfrak{A} \longrightarrow \mathbb{C}$ by

$$
D_{T, \varphi}(x, y)=\varphi\left(T\left(x^{*} y\right)-T(x)^{*} T(y)\right), \quad x, y \in \mathfrak{A} .
$$

Then it is positive by Kadison's inequality, and by the Cauchy-Schwarz inequality we have $D_{T, \varphi}(x, y)=0$ for all $y \in \mathfrak{A}$ if and only if $D_{T, \varphi}(x, x)=0$, i. e. $T\left(x^{*} y\right)=T(x)^{*} T(y)$ for all $y \in \mathfrak{A}$ if and only if $T\left(x^{*} x\right)=T(x)^{*} T(x)$. Thus it follows that fix $S$ is a ${ }^{*}$-subalgebra. Let $\left\{x_{n}\right\}_{n \in \mathbb{N}} \subseteq$ fix $S$ be a 
sequence with $\lim x_{n}=x$, then for $T \in S$ it follows that $\|T(x)-x\|=$ $\lim _{n \rightarrow \infty}\left\|T(x-n)-x_{n}\right\|=0$, hence $x \in$ fix $S$.

The next result was fist observed in [71] in the case of a quantum dynamical semigroup.

Lemma 1.18 Let $\mathfrak{M}$ be a von Neumann algebra and $S \subseteq \mathrm{L}(\mathfrak{M})$ a family of strongly positive normal contractive linear maps on $\mathfrak{M}$. Suppose that $\Phi$ is a faithful family of normal subinvariant states for $S$. Then fix $S$ is a von Neumann subalgebra of $\mathfrak{M}$.

Proof. By Lemma 1.17 fix $S$ is a $\mathrm{C}^{*}$-subalgebra. By the definition of fix $S$ and the normality of $S$ it is ultraweakly closed and hence a von Neumann subalgebra.

\subsubsection{Almost Periodicity}

In this section we establish some well-known relations between equicontinuity and almost periodicity of semigroups on von Neumann algebras. We will use the $\sigma\left(\mathrm{L}(\mathfrak{M}), \mathfrak{M} \otimes_{\gamma} \mathfrak{M}_{*}\right)$-topology on $\mathrm{L}(\mathfrak{M})$, which we introduce and study now.

Let $X_{1}$ and $X_{2}$ be two normed spaces and denote their algebraic tensor product by $\mathrm{X}_{1} \odot \mathrm{X}_{2}$ and the set of all bounded linear operators from $\mathrm{X}_{1}$ to $\mathrm{X}_{2}$ by $\mathrm{L}\left(\mathrm{X}_{1}, \mathrm{X}_{2}\right)$. A norm $\gamma$ on $\mathrm{X}_{1} \odot \mathrm{X}_{2}$ is called a cross norm if it satisfies the condition

$$
\gamma\left(x_{1} \otimes x_{2}\right)=\left\|x_{1}\right\| \cdot\left\|x_{2}\right\|, \quad \text { for all } x_{1} \in \mathbf{X}_{1}, x_{2} \in \mathbf{X}_{2} .
$$

An important cross norm is defined by

$$
\gamma(x)=\inf \left\{\sum_{i=1}^{n}\left\|x_{i}^{(1)}\right\| \cdot\left\|x_{i}^{(2)}\right\|: x=\sum_{i=1}^{n} x_{i}^{(1)} \otimes x_{i}^{(2)} \in \mathrm{X}_{1} \odot \mathrm{X}_{2}\right\},
$$

it is called the greatest or projective cross norm, henceforth denoted by $\gamma$. The completion of $\mathbf{X}_{1} \odot \mathbf{X}_{2}$ with respect to $\gamma$ is denoted by $\mathbf{X}_{1} \otimes_{\gamma} \mathbf{X}_{2}$. Any cross norm $\beta$ induces a norm $\beta^{*}$ on $\mathrm{X}_{1}^{*} \odot \mathrm{X}_{2}^{*}$ by

$$
\beta^{*}(\varphi)=\sup \left\{\langle x, \varphi\rangle: x \in \mathrm{X}_{1} \odot \mathrm{X}_{2}, \beta(x) \leq 1\right\},
$$

where, if $\varphi=\sum_{i=1}^{n} \varphi_{i}^{(1)} \otimes \varphi_{i}^{(2)} \in \mathrm{X}_{1}^{*} \odot \mathrm{X}_{2}^{*}$ and $x=\sum_{j=1}^{m} x_{j}^{(1)} \otimes x_{j}^{(2)} \in$ $\mathrm{X}_{1} \odot \mathrm{X}_{2}$, we have

$$
\langle x, \varphi\rangle=\sum_{i=1}^{n} \sum_{j=1}^{m}\left\langle x_{j}^{(1)}, \varphi_{i}^{(1)}\right\rangle\left\langle x_{j}^{(2)}, \varphi_{i}^{(2)}\right\rangle .
$$


It can be shown (see [30], Proposition IV.2.2.) that $\gamma^{*}$ is also a cross norm on $\mathbf{X}_{1}^{*} \odot \mathbf{X}_{2}^{*}$. Next, if $x^{(1)} \in \mathbf{X}_{1}$ and $x^{(2)} \in \mathbf{X}_{2}$ then for $\varphi \in\left(\mathbf{X}_{1} \otimes_{\gamma} \mathbf{X}_{2}\right)^{*}$ we have $\left|\left\langle x^{(1)} \otimes x^{(2)}, \varphi\right\rangle\right| \leq\left\|x^{(1)}\right\| \cdot\left\|x^{(2)}\right\| \cdot\|\varphi\|$, thus we can define bounded linear operators $\phi_{1}^{\prime}(\varphi): \mathrm{X}_{1} \longrightarrow \mathrm{X}_{2}^{*}$ and $\phi_{2}^{\prime}(\varphi): \mathrm{X}_{2} \longrightarrow \mathrm{X}_{1}^{*}$ by

$$
\left\langle x^{(1)} \otimes x^{(2)}, \varphi\right\rangle=\left\langle x^{(2)}, \phi_{1}^{\prime}(\varphi)\left(x^{(1)}\right)\right\rangle=\left\langle x^{(1)}, \phi_{2}^{\prime}(\varphi)\left(x^{(2)}\right)\right\rangle .
$$

We then have the following result (see Theorem III.2.3 of [30]).

Theorem 1.19 The maps $\phi_{1}^{\prime}:\left(\mathrm{X}_{1} \otimes_{\gamma} \mathrm{X}_{2}\right)^{*} \longrightarrow \mathrm{L}\left(\mathrm{X}_{1}, \mathrm{X}_{2}^{*}\right)$ and $\phi_{2}^{\prime}:\left(\mathrm{X}_{1} \otimes_{\gamma}\right.$ $\left.\mathrm{X}_{2}\right)^{*} \longrightarrow \mathrm{L}\left(\mathrm{X}_{2}, \mathrm{X}_{1}^{*}\right)$ are bijective isometries.

We will apply these results to the pointwise ultraweak topology on a von Neumann algebra. Therefore let $\mathfrak{M}$ be a von Neumann algebra and specialize to the case $\mathrm{X}_{1}=\mathfrak{M}$ and $\mathrm{X}_{2}=\mathfrak{M}_{*}$, for which $\mathrm{L}(\mathfrak{M})=\mathrm{L}\left(\mathrm{X}_{1}, \mathrm{X}_{2}^{*}\right)$. Choose $T \in \mathrm{L}(\mathfrak{M})$, then there exists $\psi \in\left(\mathfrak{M} \otimes_{\gamma} \mathfrak{M}_{*}\right)^{*}$ such that $\phi_{1}^{\prime}(\psi)=T$. For all $x \in \mathfrak{M}$ and $\varphi \in \mathfrak{M}_{*}$ we have by the definition of $\phi_{1}^{\prime}$

$$
\begin{aligned}
|\langle\psi, x \otimes \varphi\rangle| & =\left|\left\langle\varphi, \phi_{1}^{\prime}(\psi)(x)\right\rangle\right|=|\langle\varphi, T(x)\rangle| \\
& =p_{x, \varphi}(T),
\end{aligned}
$$

and we see that the family of seminorms $\left\{p_{x, \varphi}: x \in \mathfrak{M}, \varphi \in \mathfrak{M}_{*}\right\}$ generates the pointwise $\sigma\left(\mathfrak{M}, \mathfrak{M}_{*}\right)$ topology on $\mathrm{L}(\mathfrak{M})$. Consequently, this topology agrees with the $\sigma\left(\mathrm{L}(\mathfrak{M}), \mathfrak{M} \otimes_{\gamma} \mathfrak{M}_{*}\right)$ topology on $\mathrm{L}(\mathfrak{M})$ which is thus a weak* topology. In particular, since $\mathrm{L}(\mathfrak{M}) \cong\left(\mathfrak{M} \otimes_{\gamma} \mathfrak{M}_{*}\right)^{*}$, it follows byAlaoglu's theorem that ball $\mathrm{L}(\mathfrak{M})$ is $\sigma\left(\mathrm{L}(\mathfrak{M}), \mathfrak{M} \otimes_{\gamma} \mathfrak{M}_{*}\right)$ compact. This fact will be used repeatedly in this work. We also consider the pointwise $\sigma\left(\mathfrak{M}_{*}, \mathfrak{M}\right)$ topology on $\mathrm{L}\left(\mathfrak{M}_{*}\right)$. Observe that $T \in \mathrm{L}(\mathfrak{M})$ has a predual $T_{*}$ in $\mathrm{L}\left(\mathfrak{M}_{*}\right)$ if and only if $T$ is ultraweakly continuous, i.e. if and only if its dual $T^{*}$ has the property $T^{*}\left(\mathfrak{M}_{*}\right) \subseteq \mathfrak{M}_{*}$. We define a dual pairing between $\mathrm{L}\left(\mathfrak{M}_{*}\right)$ and $\mathfrak{M} \odot \mathfrak{M}_{*}$ by $\left\langle T_{*}, x \otimes \varphi\right\rangle=\left\langle x, T_{*}(\varphi)\right\rangle=\langle T(x), \varphi\rangle$ for all $x \otimes \varphi \in \mathfrak{M} \odot \mathfrak{M}_{*}$. Then the pointwise $\sigma\left(\mathfrak{M}_{*}, \mathfrak{M}\right)$ topology is the $\sigma\left(\mathrm{L}\left(\mathfrak{M}_{*}\right), \mathfrak{M} \odot \mathfrak{M}_{*}\right)$ topology.

The following result has been established in [87].

Lemma 1.20 Let $\mathfrak{M}$ be a von Neumann algebra and let $S \subseteq$ ball $\mathrm{L}(\mathfrak{M})$ be a family of normal contractive linear maps. The following assertions are equivalent:

1. The set $\left\{T_{*}: T \in S\right\} \subseteq \mathrm{L}(\mathfrak{M})_{*}$ is $\sigma\left(\mathrm{L}\left(\mathfrak{M}_{*}\right), \mathfrak{M} \odot \mathfrak{M}_{*}\right)$ relatively compact.

2. For each $\varphi \in \mathfrak{M}_{*}$ the set $\left\{T_{*}(\varphi): T \in S\right\}$ is $\sigma\left(\mathfrak{M}_{*}, \mathfrak{M}\right)$ relatively compact. 
3. For each $\sigma\left(\mathfrak{M}_{*}, \mathfrak{M}\right)$ compact subset $K$ of $\mathfrak{M}_{*}$ the set $\left\{T_{*}(\varphi): T \in\right.$ $S, \varphi \in K\}$ is $\sigma\left(\mathfrak{M}_{*}, \mathfrak{M}\right)$ relatively compact.

4. $S$ is equicontinuous on $\mathfrak{M}$ when $\mathfrak{M}$ is endowed with the $\tau\left(\mathfrak{M}, \mathfrak{M}_{*}\right)$ topology.

5. $\bar{S}$ consists of normal operators, where the closure is taken with respect to the $\left.\sigma\left(\mathrm{L}(\mathfrak{M}), \mathfrak{M} \otimes_{\gamma} \mathfrak{M}_{*}\right)\right)$ topology.

Proof. The implication 1. $\Rightarrow 2$. is immediate. For 2. $\Rightarrow 3$. it suffices to check that $S_{*}(K)=\left\{T_{*}(\varphi): T \in S, \varphi \in K\right\}$ is relatively sequentially compact in view of the the Eberlein-Šmulian theorem. To this end let $\left\{\psi_{n}\right\}_{n \in \mathbb{N}}$ be a sequence in $S_{*}(K)$, i. e. $\psi_{n}=\left(T_{n}\right)_{*}\left(\varphi_{n}\right)$ for some $\varphi_{n} \in K$ and $T_{n} \in S$. By compactness there exists a subsequence $\left\{\varphi_{n}(k)\right\}_{k \in \mathbb{N}}$ such that $\lim _{k \rightarrow \infty} \varphi_{n(k)}=\varphi$. Thus it suffices to check that $\left\{T_{*}\left(\varphi_{n}\right): T \in S, n \in \mathbb{N}\right\}$ is sequentially compact relative to $\sigma\left(\mathfrak{M}_{*}, \mathfrak{M}\right)$ for any $\sigma\left(\mathfrak{M}_{*}, \mathfrak{M}\right)$-converging sequence $\left\{\varphi_{n}\right\}_{n \in \mathbb{N}} \subseteq \mathfrak{M}_{*}$. Choose projections $\left\{p_{k}\right\}_{k \in \mathbb{N}}$ in $\mathscr{P}(\mathfrak{M})$ such that $p_{k} \perp p_{\ell}$ if $k \neq \ell$ and such that $p_{k} \downarrow 0$ as $k \rightarrow \infty$. We claim that $\lim _{k \rightarrow \infty}\left\langle T\left(p_{k}\right), \varphi_{n}\right\rangle=0$ uniformly for $T \in S$ and $n \in \mathbb{N}$, by a result of Akemann [35] this will imply relative compactness of $\left\{T_{*}\left(\varphi_{n}\right): T \in T, n \in \mathbb{N}\right\}$ and hence 3. Put $\lim _{n \rightarrow \infty} \varphi_{n}=\varphi$ and let $\epsilon>0$. Define the sets

$$
Q_{n}=\left\{x \in \text { ball } \mathfrak{M}:\left|\left\langle x, \varphi_{m}-\varphi\right\rangle\right| \leq \epsilon / 4 \text { for all } m \geq n\right\}, \quad n \in \mathbb{N} .
$$

Then each $Q_{n}$ is $\sigma\left(\mathfrak{M}, \mathfrak{M}_{*}\right)$ closed and $\bigcup_{n=1}^{\infty} Q_{n}=$ ball $\mathfrak{M}$. Since ball $\mathfrak{M}$ is $\sigma\left(\mathfrak{M}, \mathfrak{M}_{*}\right)$ compact, Baire's theorem applies and it follows that there exists $n_{0}$ such that $Q_{n_{0}}$ contains an interior point $x_{0}$. This means there exist $\psi_{1}, \ldots, \psi_{r} \in \mathfrak{M}_{*}$ such that

$$
x_{0}+\left\{x \in \text { ball } \mathfrak{M}:\left|\left\langle x, \psi_{i}\right\rangle\right|<1, i=1, \ldots, r\right\} \subseteq Q_{n_{0}} .
$$

Since the sets $\left\{T_{*}\left(\psi_{i}\right): T \in S\right\}$ are relatively compact there exists $k_{1} \in \mathbb{N}$ such that $\left|\left\langle T\left(p_{k}\right), \psi_{i}\right\rangle\right|<1$ if $k \geq k_{1}$ and $T \in S, i=1, \ldots, r$, hence $T\left(p_{k}\right)+x_{0} \in Q_{n_{0}}$ for $k \geq k_{1}$. Since $x_{0}$ lies in $Q_{n_{0}}$ we obtain $\mid\left\langle T\left(p_{k}\right), \varphi_{m}-\right.$ $\varphi\rangle \mid<\epsilon / 2$ if $k \geq k_{1}, m \geq m_{0}$, and $T \in S$. By assumption the set $\left\{T_{*}\left(\varphi_{m}-\varphi\right): T \in S, 1 \leq m \leq n_{0}-1\right\}$ is relatively compact with respect to $\sigma\left(\mathfrak{M}_{*}, \mathfrak{M}\right)$, thus there exists $k_{2} \in \mathbb{N}$ such that $\left|\left\langle T\left(p_{k}\right), \varphi_{m}-\varphi\right\rangle\right|<\epsilon / 2$ if $k \geq k_{2}$ and $T \in S, 1 \leq m \leq n_{0}-1$. Finally, there exists $k_{3} \in \mathbb{N}$ such that $\left|\left\langle T\left(p_{k}\right), \varphi\right\rangle\right|<\epsilon / 2$ if $k \geq k_{3}$ and $T \in S$. If $k_{0}=\max \left\{k_{1}, k_{2}, k_{3}\right\}$ then we conclude that $\left|\left\langle T\left(p_{k}\right), \varphi_{m}\right\rangle\right|<\epsilon$ if $k \geq k_{0}$ and $T \in S, m \in \mathbb{N}$, thus 3 . is satisfied. 
For the proof of $3 . \Rightarrow 4$. let $K$ be a convex and $\sigma\left(\mathfrak{M}, \mathfrak{M}_{*}\right)$ compact subset of $\mathfrak{M}_{*}$. Let $p_{K}=\sup \{|\langle x, \psi\rangle|: \psi \in K\}$ be the corresponding seminorm of the $\tau\left(\mathfrak{M}, \mathfrak{M}_{*}\right)$-topology. By assumption the set $K^{\prime}=\overline{\operatorname{co}}\left\{T_{*}(\varphi): T \in\right.$ $S, \varphi \in K\}$ is $\sigma\left(\mathfrak{M}_{*}, \mathfrak{M}\right)$ compact and convex, and

$$
p_{K}(T(x))=\sup _{\psi \in K}|\langle T(x), \psi\rangle| \leq \sup _{\psi \in K^{\prime}}|\langle x, \psi\rangle|=p_{K^{\prime}}(x),
$$

which is equicontinuity.

To establish 4. $\Rightarrow 5$. let $T \in \bar{S}$ and let $\left\{T_{i}\right\}_{i \in I} \subseteq S$ be a net with $\lim _{i \rightarrow \infty} T_{i}=T$ relative to $\sigma\left(\mathrm{L}(\mathfrak{M}), \mathfrak{M}_{*} \otimes_{\gamma} \mathfrak{M}\right)$. Choose $\varphi \in \mathfrak{M}_{*}$ and let $K=\{\varphi\}$. By assumption there exist a compact convex subset $K^{\prime}$ of $\mathfrak{M}_{*}$ and $C \geq 0$ such that $\left|\left\langle T_{i}(x), \varphi\right\rangle\right|=p_{K}(x) \leq C p_{K^{\prime}}(x)$ for any $x \in \mathfrak{M}$. Letting $i \rightarrow \infty$ shows $|\langle T(x), \varphi\rangle| \leq C p_{K^{\prime}}(x)$, thus $x \mapsto\langle T(x), \varphi\rangle$ is normal for any $\varphi \in \mathfrak{M}_{*}$ and 5 . follows.

To prove $5 . \Rightarrow 1$. choose a net $\left\{\left(T_{i}\right)_{*}\right\}_{i \in I} \subseteq S_{*}$. By compactness of ball $\mathrm{L}(\mathfrak{M})$ there exists a subnet $\left\{T_{i(j)}\right\}_{j \in J}$ such that $\lim _{j \rightarrow \infty} T_{i(j)}=T$ relative to $\sigma\left(\mathrm{L}(\mathfrak{M}), \mathfrak{M}_{*} \otimes_{\gamma} \mathfrak{M}\right)$. Since $T$ is normal by assumption it has a predual $T_{*}$ and it follows that $\lim _{j \rightarrow \infty}\left(T_{i(j)}\right)_{*}=T_{*}$ relative to $\sigma\left(\mathrm{L}\left(\mathfrak{M}_{*}\right), \mathfrak{M} \otimes \mathfrak{M}_{*}\right)$, hence $S$ is relatively compact in the appropriate topology.

In case $S$ in Lemma 1.20 is a semigroup one reserves a special name for the equivalent properties of the lemma.

Definition 1.21 A semigroup $S$ of normal contractive operators on a von Neumann algebra $\mathfrak{M}$ is called ultraweakly almost periodic if the properties of Lemma 1.20 are satisfied.

Next we give a sufficient criterion for the properties of Lemma 1.20, up to some modifications the proof is due to [87].

Proposition 1.22 Let $\mathfrak{M}$ be a von Neumann algebra and let $S \subseteq \mathrm{L}(\mathfrak{M})_{1}$ be a family of normal contractive linear maps. If $\Phi$ is a faithful set of normal states on $\mathfrak{M}$ such that

$$
\varphi\left(T(x)^{*} T(x)\right) \leq \varphi\left(x^{*} x\right) \quad \text { for all } x \in \mathfrak{M}, \varphi \in \Phi, T \in S,
$$

then $S$ satisfies the equivalent properties of Lemma 1.20.

Proof. Let $\psi$ be a normal state on $\mathfrak{M}$ and assume for a contradiction that $\left\{T_{*}(\psi): T \in S\right\}$ is not $\sigma\left(\mathfrak{M}_{*}, \mathfrak{M}\right)$ relatively compact. Then there exists $\epsilon>0$ and a sequence $\left\{p_{n}\right\}_{n \in \mathbb{N}}$ of projections with $p_{k} \perp p_{\ell}$ for $k \neq \ell$ such that $p_{k} \downarrow 0$ as $k \rightarrow \infty$, and a sequence $\left\{T_{n}\right\}_{n \in \mathbb{N}} \subseteq S$ such that 
$\left|\left\langle T_{n}\left(p_{n}\right), \psi\right\rangle\right|>\epsilon$ for all $n \in \mathbb{N}$. Let $\left(\pi_{\varphi}, \mathscr{H}_{\varphi}, \xi_{\varphi}\right)$ be the GNS triplet of $\varphi \in \Phi$ and put

$$
\pi=\bigoplus_{\varphi \in \Phi} \pi_{\varphi}, \quad \mathscr{H}=\bigoplus_{\varphi \in \Phi} \mathscr{H}_{\varphi} .
$$

Then $\pi$ is a faithful normal representation of $\mathfrak{M}$ on $\mathscr{H}$ and the set $\left\{x \xi_{\varphi}\right.$ : $\left.x \in \pi(\mathfrak{M})^{\prime}, \varphi \in \Phi\right\}$ is total in $\mathscr{H}$. Now for $\varphi \in \Phi$ and $x \in \pi(\mathfrak{M})^{\prime}$ it follows by (1.42) that

$$
\begin{aligned}
\left\|\pi\left(T_{n}\left(p_{n}\right)\right) x \xi_{\varphi}\right\| & =\left\|x \pi\left(T_{n}\left(p_{n}\right)\right) \xi_{\varphi}\right\| \\
& \leq\|x\| \cdot\left\|\pi\left(p_{n}\right) \xi_{\varphi}\right\| \\
& =\|x\| \cdot\left\langle\pi\left(T_{n}\left(p_{n}\right)\right) \xi_{\varphi}, \pi\left(T_{n}\left(p_{n}\right)\right) \xi_{\varphi}\right\rangle^{1 / 2} \\
& =\|x\| \cdot \varphi\left(T_{n}\left(p_{n}\right)^{*} T_{n}\left(p_{n}\right)\right)^{1 / 2} \\
& \leq\|x\| \cdot \varphi\left(p_{n}\right)^{1 / 2} \rightarrow 0
\end{aligned}
$$

as $n \rightarrow \infty$, hence it follows that $T_{n}\left(p_{n}\right) \rightarrow 0$ in the strong operator topology, contradiction.

\subsubsection{Amenability and Mean Ergodicity}

In this section we establish a number of implications of amenability of semigroups of maps on a von Neumann algebra. We start by introducing some terminology. Let $S$ be a semigroup and denote the Banach space of all bounded complex functions on $S$ by $\mathrm{B}(S)$, equipped with the supnorm. Let $\mathrm{F} \subseteq \mathrm{B}(S)$ be a subspace. A mean on $\mathrm{F}$ is a linear functional $m \in \mathrm{F}^{*}$ such that $m(f) \geq 0$ if $f \geq 0$ and such that $\|m\|=1$. If $\ell_{g}(\mathrm{~F}) \subseteq \mathrm{F}$ and $m\left(\ell_{g}(f)\right)=m(f)$ for all $f \in \mathrm{F}\left(r_{g}(\mathrm{~F}) \subseteq \mathrm{F}\right.$ and $m\left(r_{g}(f)\right)=m(f)$ for all $f \in \mathrm{F}$ ) for any $g \in S$ then $m$ is called left (right) invariant. Here $\ell_{g}(f)=g(f \cdot)$ and $r_{g}(f)=f(\cdot g)$. If $m$ is both left and right invariant, then it is called invariant. The semigroup $S$ is called left (right) amenable if there exists a left (right) invariant mean on $\mathrm{B}(S)$, and $S$ is called amenable if there exists a left and a right invariant mean on $\mathrm{B}(S)$. If $S$ is amenable, then it follows that there exists an invariant mean on $\mathrm{B}(S)$.

We start with a lemma that is related to Day's fixed point theorem for topological semigroups and well-known to experts in topological semigroups. The set of all continuous affine functions on a convex set $K$ is denoted by $\mathrm{A}(K)$.

Lemma 1.23 Let $S$ be a locally compact semitopological semigroup. Assume that $S$ acts on a convex compact subset $K$ of a locally convex topological vector space $E$, i. e. $S \times K \ni(g, x) \mapsto g . x \in K$, such that the action 
is affine,

$$
g \cdot(\lambda x+\mu y)=\lambda g \cdot x+\mu g \cdot y, \quad g \in S, \lambda, \mu \geq 0, \lambda+\mu=1, x, y \in K,
$$

and moreover separately continuous. Let $m$ be a mean on $\mathrm{B}(S)$ and for $f \in \mathrm{A}(K)$ define $\phi_{f}: S \longrightarrow \mathbb{C}$ by $\phi_{f}(g)=f\left(g . x_{0}\right)$ where $x_{0} \in K$ is fixed. Then there exists $x \in K$ such that $m\left(\phi_{f}\right)=f(x)$ for all $f \in \mathrm{A}(K)$.

Proof. First notice that $\phi_{f} \in \mathrm{C}_{\mathrm{b}}(S)$. Let $M \subseteq \mathrm{C}_{\mathrm{b}}(S)^{*}$ be the set of all means on $\mathrm{C}_{\mathrm{b}}(S)$; then by restriction, $m \in \mathrm{C}_{\mathrm{b}}(S)^{*}$. By the bipolar theorem $M \subseteq\left\{\delta_{g}: g \in S\right\}^{\circ \circ}=\overline{\mathrm{co}}\left(\left\{\delta_{g}: g \in S\right\} \cup\{0\}\right)$, where the closure is taken relative to $\sigma\left(\mathrm{C}_{\mathrm{b}}(S)^{*}, \mathrm{C}_{\mathrm{b}}(S)\right)$. Here $\mathrm{C}_{\mathrm{b}}(S) \ni f \mapsto\left\langle f, \delta_{g}\right\rangle=f(g)$ defines a mean on $\mathrm{C}_{\mathrm{b}}(S)$. Hence there exists a net $\left\{m_{i}^{\prime}\right\}_{i \in I} \subseteq \operatorname{co}\left(\left\{\delta_{g}: g \in S\right\} \cup\{0\}\right)$ such that $m_{i}^{\prime} \rightarrow m$ relative to the $\sigma\left(\mathrm{C}_{\mathrm{b}}(S)^{*}, \mathrm{C}_{\mathrm{b}}(S)\right)$ topology. Define $m_{i}=m_{i}^{\prime} /\left\|m_{i}^{\prime}\right\|$, then $m_{i}$ is normalized, i.e. $m_{i} \in \operatorname{co}\left\{\delta_{g}: g \in S\right\}$ and $\left\|m_{i}^{\prime}\right\|=m_{i}^{\prime}(1) \rightarrow m(1)=1$, i. e. $m_{i} \rightarrow m$. This means that each $m_{i}$ is of the form $m_{i}=\sum_{k=1}^{n} \lambda_{k} \delta_{g_{k}}, \lambda_{1}, \ldots, \lambda_{n} \geq 0, \sum_{k=1}^{n} \lambda_{k}=1, g_{1}, \ldots, g_{n} \in S$, and we have

$$
m_{i}\left(\phi_{f}\right)=\sum_{k=1}^{n} \lambda_{k} \phi_{f}\left(g_{k}\right)=\sum_{k=1}^{n} \lambda_{k} f\left(g . x_{0}\right)=f\left(x_{i}\right),
$$

where

$$
x_{i}=\sum_{k=1}^{n} \lambda_{k} g_{k} \cdot x_{0} \in K
$$

By compactness of $K$ we can assume (after passing to a subnet) that the net $\left\{x_{i}\right\}_{i \in I}$ converges: $\lim _{i \rightarrow \infty} x_{i}=: x \in K$, hence

$$
m\left(\phi_{f}\right)=\lim _{i \rightarrow \infty} m_{i}\left(\phi_{f}\right)=\lim _{i \rightarrow \infty} f\left(x_{i}\right)=f(x)
$$

for all $f \in \mathrm{A}(K)$, as was to be shown.

The next result is contained, e.g., in [126], here we give a proof using Lemma 1.23.

Proposition 1.24 Let $S$ be a semigroup of normal contractive operators on a von Neumann algebra $\mathfrak{M}$ such that $T(\mathbb{1})=\mathbb{1}$ for all $T \in S$. Suppose that the following conditions are satisfied:

1. $\overline{\mathrm{co}} S$ is amenable.

2. $S$ is weak* almost periodic. 
3. $T\left(p_{\varphi}\right)=p_{\varphi}$ for all $T \in S$ whenever $\varphi$ is a normal invariant state under $S$ and $p_{\varphi}$ is its support projection.

Then there exists an orthogonal faithful family of invariant states for $S$.

Proof. Let $\varphi$ be a normal state on $\mathfrak{M}$, then $K=\left\{T_{*}(\varphi): T \in \overline{\operatorname{co}} S\right\}=$ $\overline{\operatorname{co}}\left\{T_{*}(\varphi): T \in S\right\}$ is $\sigma\left(\mathfrak{M}_{*}, \mathfrak{M}\right)$ compact as a consequence of assumption 2 and consists of states since $T(\mathbb{1})=\mathbb{1}$. Define an affine action of $S$ on $K$ by

$$
S \times K \ni(T, \psi) \mapsto T . \psi=T_{*}(\psi) \in K,
$$

and let $\phi_{f}(T)=f(T . \varphi)=f\left(T_{*}(\varphi)\right)$ where $f \in \mathrm{A}(K)$. Since $\overline{\operatorname{co}} S$ is compact, by Lemma 1.23 there exists $\mu \in K$ such that $m\left(\phi_{f}\right)=f(\mu)$ for all $f \in \mathrm{A}(K)$, where $m$ is an invariant mean of $\overline{\mathrm{co}} S$. With $r_{T} \phi_{f}=\phi_{f \circ T_{*}}$ this implies

$$
f\left(T_{*}(\mu)\right)=f(T . \mu)=m\left(r_{T} \phi_{f}\right)=m\left(\phi_{f}\right)=f(\mu) \text { for any } f \in \mathrm{A}(K),
$$

hence $\mu \circ T=\mu$ for all $T \in S$ and $\mu$ is an invariant state of $S$. Now let $x \in$ fix $S$ and define $K \ni \psi \mapsto f(\psi)=\langle x, \psi\rangle$. Then $f \in \mathrm{A}(K)$ and $\phi_{f}(T)=\langle x, \varphi\rangle$ for any $T \in S$. Hence $\langle x, \varphi\rangle=m\left(\phi_{f}\right)=f(\mu)=\langle x, \mu\rangle$, i. e. $\varphi$ and $\mu$ agree on fix $S$.

Now let $\Omega$ be a maximal orthogonal family of normal invariant states for $S$ and put $q=\mathbb{1}-\sum_{\omega \in \Omega} p_{\omega}$. Then $T(q)=q$ for every $T \in S$ by assumption 3. Suppose that $q \neq 0$ and let $\omega$ be a normal state such that $\omega(q)=1$. Then by the preceding paragraph we find a faithful normal state $\mu$ which is invariant for $S$ such that it agrees with $\omega$ on fix $S$, in particular $\mu(q)=\omega(q)=1$, thus $p_{\mu} \leq q \perp p_{\omega}, \omega \in \Omega$, so that $\Omega$ is not maximal. Thus $q=0$ and $\Omega$ must be faithful.

Corollary 1.25 Suppose that the assumptions of Proposition 1.24 hold true and assume additionally that $\mathfrak{M}$ is $\sigma$-finite. Then there exists a normal invariant state for $S$.

Proof. By $\sigma$-finiteness an orthogonal set of faithful normal states must be countable. Let $\left\{\omega_{n}: n \in \mathbb{N}\right\}$ be such a set and define the state

$$
\varphi=\sum_{n=1}^{\infty} \frac{1}{2^{n}} \omega_{n} .
$$

Then $\varphi$ is normal by Corollary III.5.2. of [30], and moreover faithful and invariant for $S$.

We now prove a converse of Proposition 1.24, the proof follows [126]. 
Proposition 1.26 Let $S$ be a semigroup of normal contractive and unital linear operators on a von Neumann algebra $\mathfrak{M}$. Suppose that there exists a faithful family $\Phi$ of normal states on $\mathfrak{M}$ such that (1.42) holds for all $T \in S$ and $\varphi \in \Phi$. Then there exists a normal projection $E$ on $\mathfrak{M}$ onto the fixed point subspace fix $S$ such that $E \circ T=T \circ E$ for all $T \in S$.

Proof. If $\lambda_{1}, \ldots, \lambda_{n} \geq 0$ such that $\lambda_{1}+\cdots+\lambda_{n}=1$ and $T_{1}, \ldots, T_{n} \in S$ we have by the Cauchy-Schwarz inequality

$$
\begin{aligned}
\varphi\left[\left(\sum_{i=1}^{n} \lambda_{i} T_{i}(x)^{*}\right)\left(\sum_{j=1}^{n} \lambda_{j} T_{j}(x)\right)\right]=\sum_{i, j=1}^{n} \lambda_{i} \lambda_{j} \varphi\left(T_{i}(x)^{*} T_{j}(x)\right) \\
\leq \sum_{i, j=1}^{n} \lambda_{i} \lambda_{j} \varphi\left(T_{i}(x)^{*} T_{i}(x)\right)^{1 / 2} \varphi\left(T_{j}(x)^{*} T_{j}(x)\right)^{1 / 2} \\
\leq \varphi\left(x^{*} x\right)
\end{aligned}
$$

for any $x \in \mathfrak{M}$ and $\varphi \in \Phi$, i.e. (1.42) extends to $\operatorname{co} S$ and also to the pointwise ultraweak closure $\overline{c o} S$ since it agrees with the pointwise strong closure by convexity. For $\varphi \in \Phi$ let $\mathscr{H}_{\varphi}$ be the real Hilbert space obtained by completing $\mathfrak{M}^{\mathrm{sa}} p_{\varphi}$, where $p_{\varphi}$ is the support projection of $\varphi$, with respect to the real inner product

$$
\left\langle x p_{\varphi}, y p_{\varphi}\right\rangle_{\varphi}=\varphi\left(p_{\varphi} x y p_{\varphi}\right)=\varphi(x y), \quad \text { for all } x, y \in \mathfrak{M}^{\mathrm{sa}},
$$

and define the Hilbert space

$$
\mathscr{H}=\bigoplus_{\varphi \in \Phi} \mathscr{H}_{\varphi}
$$

To every $T \in S$ we associate the operator $T_{\varphi}$ on $\mathscr{H}_{\varphi}$, defined by $T_{\varphi}\left(x p_{\varphi}\right)=$ $T(x) p_{\varphi} \in \mathscr{H}_{\varphi}$. Now we can define the map $i: \overline{\operatorname{co}} S \longrightarrow$ ball $\mathrm{L}(\mathscr{H})$ by

$$
i(T)=\bigoplus_{\varphi \in \Phi} T_{\varphi} \in \mathrm{L}(\mathscr{H})
$$

Then $i$ is a semigroup homomorphism and moreover it is affine and satisfies $\left\langle x p_{\varphi}, i(T) p_{\varphi}\right\rangle=\left\langle x p_{\varphi}, T(y) p_{\varphi}\right\rangle_{\varphi}=\varphi(x T(y))$ for all $x, y \in \mathfrak{M}^{\mathrm{sa}}$ and $\varphi \in \Phi$. Thus $i$ is point weak-weak continuous and hence $i(\overline{\mathrm{co}} S)$ is ultraweakly closed by compactness of $\overline{c o} S$. Applying the Alaoglu-Birkhoff mean ergodic theorem to $i(\overline{\mathrm{co}} S)$ we see that there is $E \in \overline{\mathrm{co}} S$ such that $E^{2}=E$ and $E \circ T=T \circ E$ for all $T \in S$. 
Corollary 1.27 Suppose that all assumptions of Proposition 1.26 are satisfied, and assume additionally that $S$ consists of strongly positive operators. Then there exists a normal conditional expectation $E$ on $\mathfrak{M}$ onto the fixed point subalgebra fix $S$. Moreover, we have $\varphi \circ E \leq \varphi$ for all $\varphi \in \Phi$.

Proof. By strong positivity and contractivity, $\|T\| \leq 1$, it follows from Kadison's inequality that $\varphi(T(x) * T(x)) \leq \varphi\left(T\left(x^{*} x\right)\right) \leq \varphi\left(x^{*} x\right)$, so Proposition 1.26 applies. Since $S$ is positive, $\overline{\mathrm{co}} S$ is so as well, and since $E(\mathbb{1})=\mathbb{1}$ we get $\|E\|=1$, moreover fix $S$ is a von Neumann subalgebra by Lemma 1.18 , thus $E$ is a conditional expectation by Tomiyama's theorem. Since $E T E(x)=T E(x)$ we get $E(\mathfrak{M})=$ fix $S$. Finally, since $E \in \overline{\mathrm{co}} S$ the last statement follows as well.

The existence of a projection onto the fixed point subspace of $S$ can be regarded as a noncommutative generalization of von Neumann's mean ergodic theorem, hence the following terminology is introduced.

Definition 1.28 Let $S$ be a semigroup of normal and unital linear operators on a von Neumann algebra $\mathfrak{M}$. The semigroup $S$ is called mean ergodic if there exists a normal projection $E$ onto the fixed point subspace fix $S$ of $S$.

The role of assumption 3. in Proposition 1.24 is clarified by the following lemma by giving a certain converse of it.

Lemma 1.29 Let $S$ be a semigroup of normal contractive operators on a von Neumann algebra $\mathfrak{M}$ such that $T(\mathbb{1})=\mathbb{1}$ for all $T \in S$, and let $\Phi$ be a faithful set of normal invariant states for $S$. Then $T\left(p_{\psi}\right)=p_{\psi}$ for any $T \in S$ and every normal $S$-invariant state $\psi$.

Proof. We have $T\left(p_{\psi}\right) \leq \mathbb{1}$, i. e. $0 \leq \mathbb{1}-p_{\psi}$ and $\psi\left(\mathbb{1}-T\left(p_{\psi}\right)\right)=1-\psi\left(p_{\psi}\right)=$ 0 , consequently $\mathbb{1}-T\left(p_{\psi}\right) \leq \mathbb{1}-p_{\psi}$, so $T\left(p_{\psi}\right) \geq p_{\psi}$. Applying $\varphi \in \Phi$ we get $\varphi\left(T\left(p_{\psi}\right)-p_{\psi}\right)=0$, hence by faithfulness $T\left(p_{\psi}\right)=p_{\psi}$.

Similarly, assumption 1. of Proposition 1.24 is implied by its conclusion.

Proposition 1.30 Suppose that the assumptions of Proposition 1.26 are satisfied. Then $\overline{\mathrm{co}} S$ is amenable.

Proof. Let $E$ be the projection onto the fixed point subspace fix $S$ which exists by Proposition 1.26. We introduce the dual pairing $\langle f, T\rangle=f(T)$ for $T \in S$ and $f \in \mathrm{B}(\overline{\mathrm{co}} S)$. Define a positive linear functional $m: \mathrm{B}(\overline{\mathrm{co}} S) \longrightarrow$ $\mathbb{C}$ by $\langle m(f), T\rangle=f(E T)$ for all $T \in S$. Then for any $T^{\prime} \in S$ we have

$$
\left\langle m\left(r_{T^{\prime}}(f)\right), T\right\rangle=f\left(E T T^{\prime}\right)=f(E T)=\langle m(f), T\rangle
$$


for any $T \in S$, hence $m$ is right invariant. Similarly, it is also left invariant.

\subsubsection{Invariant and Subinvariant States}

The following result implies that for strongly positive unital maps subinvariance of a normal state entails invariance.

Proposition 1.31 Let $T: \mathfrak{M} \longrightarrow \mathfrak{M}$ be a positive linear map such that $T(\mathbb{1})=\mathbb{1}$. Assume that

$$
\varphi\left(T(x)^{*} T(x)\right) \leq \varphi\left(x^{*} x\right) \quad \text { for all } x \in \mathfrak{M}
$$

where $\varphi$ is a normal state on $\mathfrak{M}$. Then $\varphi \circ T=\varphi$.

Proof. By positivity $T$ is a contraction. Let $p_{\varphi}$ be the support projection of $\varphi$ and write $\mathfrak{M}_{\varphi}=\mathfrak{M}_{\varphi}$ as well as $\mathfrak{M}_{\varphi}^{\perp}=\mathfrak{M} p_{\varphi}^{\perp}$. By $\mathfrak{M}_{\varphi}^{\perp}=\{x \in$ $\left.\mathfrak{M}: \varphi\left(x^{*} x\right)=0\right\}$ and (1.43) we have $0 \leq \varphi\left(T(x)^{*} T(x)\right) \leq \varphi\left(x^{*} x\right)=0$ for $x \in \mathfrak{M}_{\varphi}^{\perp}$, i. e. $T\left(\mathfrak{M}_{\varphi}^{\perp}\right) \subseteq \mathfrak{M}_{\varphi}^{\perp}$. Let $x \in \mathfrak{M}$, then from $x=x p_{\varphi}+x p_{\varphi}^{\perp}$ we get

$$
T(x) p_{\varphi}=T\left(x p_{\varphi}\right) p_{\varphi}+T\left(x p_{\varphi}^{\perp}\right) p_{\varphi}=T\left(x p_{\varphi}\right) p_{\varphi}
$$

since $T\left(x p_{\varphi}^{\perp}\right) \in \mathfrak{M}_{\varphi}^{\perp}$. Now define a map $T: \mathfrak{M}_{\varphi} \longrightarrow \mathfrak{M}_{\varphi}$ by $T_{\varphi}\left(x p_{\varphi}\right)=$ $T\left(x p_{\varphi}\right) p_{\varphi}=T(x) p_{\varphi} \in \mathfrak{M}_{\varphi}$ for any $x \in \mathfrak{M}$. Moreover, define an inner product on $\mathfrak{M}_{\varphi}$ by $\left\langle x p_{\varphi}, y p_{\varphi}\right\rangle_{\varphi}=\varphi\left(p_{\varphi} x^{*} y p_{\varphi}\right)=\varphi\left(x^{*} y\right), x, y \in \mathfrak{M}$, and let $\mathscr{H}_{\varphi}$ be the Hilbert space completion of $\mathfrak{M}_{\varphi}$. Then

$$
\begin{aligned}
\left\langle T_{\varphi}\left(x p_{\varphi}\right), T_{\varphi}\left(x p_{\varphi}\right)\right\rangle_{\varphi} & =\varphi\left(T(x)^{*} T(x)\right) \\
& \leq \varphi\left(x^{*} x\right)=\varphi\left(\left(x p_{\varphi}\right)^{*} x p_{\varphi}\right) \\
& =\left\langle x p_{\varphi}, x p_{\varphi}\right\rangle_{\varphi}
\end{aligned}
$$

thus $T_{\varphi}$ extends to a contraction on $\mathscr{H}_{\varphi}$, denoted again by $T_{\varphi}$. We have $p_{\varphi}=T(\mathbb{1}) p_{\varphi}=\left(T p_{\varphi}\right) p_{\varphi}=T_{\varphi}\left(p_{\varphi}\right)$, and since $T_{\varphi}$ is a contraction, $T_{\varphi}^{*}\left(p_{\varphi}\right)=p_{\varphi}$ for the Hilbert space adjoint $T_{\varphi}^{*}$ of $T_{\varphi}$. Then we get

$$
\begin{aligned}
\varphi(T(x)) & =\varphi\left(p_{\varphi} T(x) p_{\varphi}\right)=\varphi\left(p_{\varphi} T_{\varphi}\left(x p_{\varphi}\right)\right) \\
& =\left\langle p_{\varphi}, T_{\varphi}\left(x p_{\varphi}\right)\right\rangle_{\varphi}=\left\langle T_{\varphi}^{*}\left(p_{\varphi}\right), x p_{\varphi}\right\rangle_{\varphi} \\
& =\left\langle p_{\varphi}, x p_{\varphi}\right\rangle_{\varphi}=\varphi\left(p_{\varphi} x p_{\varphi}\right) \\
& =\varphi(x)
\end{aligned}
$$

for any $x \in \mathfrak{M}$, as was to be shown. 
Corollary 1.32 Let $T: \mathfrak{M} \longrightarrow \mathfrak{M}$ be a strongly positive linear map such that $T(\mathbb{1})=\mathbb{1}$ and $\varphi(T(x)) \leq \varphi(x)$ for all $x \in \mathfrak{M}^{+}$, where $\varphi$ is a normal state on $\mathfrak{M}$. Then $\varphi \circ T=\varphi$.

Proof. By strong positivity and $\|T\| \leq 1$ we have we have $\varphi\left(T(x)^{*} T(x)\right) \leq$ $\varphi\left(T\left(x^{*} x\right)\right) \leq \varphi\left(x^{*} x\right)$, so Proposition 1.31 applies.

Remark 1.33 Under stronger assumptions we can arrive at a similar conclusion as in Proposition 1.31 by combining Proposition 1.42 and Corollary 1.32: Let $T$ be a normal positive contractive linear map on a $\sigma$-finite von Neumann algebra $\mathfrak{M}$ and suppose that $\Phi$ is a set of normal states such that (1.42) holds. Consider the semigroup $S=\left\{T^{n}: n \in \mathbb{N} \cup\{0\}\right\}$. Then $S$ is commutative, hence $\overline{c o} S$ is amenable, $S$ is weak* almost periodic, and by Lemma 1.29 we have $T\left(p_{\varphi}\right)=p_{\varphi}$ for any $\phi \in \Phi$, hence Corollary 1.32 applies and yields a normal faithful invariant state for $T$. 


\section{Chapter 2.}

\section{Decoherence: Technical Tools}

In this chapter we develop some mathematical tools which are relevant for the study of decoherence. We will consider two kinds of asymptotic splittings of quantum dynamical semigroups on a von Neumann algebra, namely the so-called isometric-sweeping splitting and the Jacobs-de Leeuw-Glicksberg splitting. The isometric-sweeping splitting is the one that is directly related to decoherence, but we indicate by an example that the Jacobs-de Leeuw-Glicksberg splitting is of relevance to decoherence in as well certain situations. Moreover, we introduce and discuss two notions of noncommutative detailed balance which both are sufficient conditions to establish the existence of the isometric-sweeping splitting.

\subsection{Mathematical Preliminaries}

\subsubsection{Tomita-Takesaki Theory}

In this section we collect the basic ingredients of Tomita-Takesaki modular theory as laid out, for example, in $[6,23]$. It serves to establish some notation and provide some results needed in the following sections.

Let $\mathfrak{M}$ be a von Neumann algebra acting on a Hilbert space $\mathscr{H}$. A subset $M \subseteq \mathscr{H}$ is called separating if for any $x \in \mathfrak{M}, x \xi=0$ for every $\xi \in M$ implies $x=0$. A subset $M$ is separating for $\mathfrak{M}$ if and only if $M$ is cyclic for $\mathfrak{M}^{\prime}$, i. e. $[\mathfrak{M} M]=\mathscr{H}$. It can be shown that the following assertions are equivalent:

1. $\mathfrak{M}$ is $\sigma$-finite, i. e. any set of mutually orthogonal projections in $\mathfrak{M}$ is countable.

2. There exists a countable separating subset $M$ of $\mathscr{H}$.

3. There exists a faithful normal state on $\mathfrak{M}$.

4. $\mathfrak{M}$ is ${ }^{*}$-isomorphic to a von Neumann algebra which admits a cyclic and separating vector. 
(See [6] for a proof.) It is easy to see that a von Neumann algebra acting on a separable Hilbert space must be $\sigma$-finite. In the following we will assume that $\mathfrak{M}$ has a cyclic and separating vector $\xi_{0}$. We define two operators

$$
\begin{array}{rlrl}
\operatorname{dom} S_{0} & =\mathfrak{M} \xi_{0} & x \xi_{0} & \mapsto S_{0}\left(x \xi_{0}\right)=x^{*} \xi_{0}, \\
\operatorname{dom} F_{0} & =\mathfrak{M}^{\prime} \xi_{0} & x^{\prime} \xi_{0} & \mapsto F_{0}\left(x^{\prime} \xi_{0}\right)=x^{\prime *} \xi_{0} .
\end{array}
$$

It can be shown that $S_{0}$ and $F_{0}$ are closable, we denote their closures by $S$ and $F$, respectively. Let

$$
S=J \Delta^{1 / 2}
$$

be the polar decomposition of $S$. The unique positive (in general unbounded) operator $\Delta$ is called the modular operator and the unique antilinear isometry $J$ is called the modular conjugation. The following relations can be verified:

$$
\begin{aligned}
& \Delta=F S, \\
& \Delta^{-1}=S F, \\
& F=J \Delta^{-1 / 2}, \\
& \Delta^{-1 / 2}=J \Delta^{1 / 2} J, \\
& J=J^{*}, \\
& J^{2}=\mathbb{1} \text {. }
\end{aligned}
$$

The key result in Tomita-Takesaki theory is the Tomita-Takesaki theorem.

Theorem 2.1 Let $\mathfrak{M}$ be a von Neumann algebra with cyclic and separating vector $\xi_{0}$. Let $\Delta$ and $J$ be the associated modular operator and modular conjugation. Then

$$
\mathfrak{M}^{\prime}=J \mathfrak{M} J
$$

and

$$
\mathfrak{M}=\Delta^{\mathrm{i} t} \mathfrak{M} \Delta^{-\mathrm{i} t} \quad \text { for all } t \in \mathbb{R} .
$$

Using (2.3) we can introduce the following group of automorphisms, which turns out to be extremely useful in the analysis of von Neumann algebras and quantum dynamical systems. Let $\omega$ be a faithful normal state on $\mathfrak{M}$ and let $\left(\pi_{\omega}, \mathscr{H}_{\omega}, \xi_{\omega}\right)$ be the associated GNS triplet, then $\pi_{\omega}$ is normal and faithful and $\xi_{\omega}$ is cyclic and separating. Let $\Delta$ and $J$ be the modular operator and modular conjugation associated with $\xi_{\omega}$ and define

$$
\sigma_{t}^{\omega}(x)=\pi_{\omega}^{-1}\left(\Delta^{\mathrm{i} t} \pi_{\omega}(x) \Delta^{-\mathrm{i} t}\right), \quad t \in \mathbb{R}, x \in \mathfrak{M} .
$$

Then $\left\{\sigma_{t}^{\omega}\right\}_{t \in \mathbb{R}}$ is an ultraweakly continuous group of automorphisms on $\mathfrak{M}$, called the modular group associated with $\omega$.

There is a connection between the existence of conditional expectations onto subalgebras and the modular group, as recorded in the following theorem due to Takesaki [124]. We will use this connection repeatedly. 
Theorem 2.2 Let $\mathfrak{M}$ be a von Neumann algebra and $\mathfrak{N} \subseteq \mathfrak{M}$ a von Neumann subalgebra. Suppose that $\omega$ is a faithful normal state on $\mathfrak{M}$. Then there exists a normal conditional expectation $E$ on $\mathfrak{M}$ onto $\mathfrak{N}$ such that $\omega \circ E=\omega$ if and only if $\mathfrak{N}$ is invariant under the modular group $\left\{\sigma_{t}^{\omega}\right\}_{t \in \mathbb{R}}$, i. e. $\sigma_{t}^{\omega}(\mathfrak{N}) \subseteq \mathfrak{N}$ for all $t \in \mathbb{R}$.

This theorem still holds if $\omega$ is replaced by a faithful normal semifinite weight which is semifinite on $\mathfrak{N}$.

\subsubsection{The KMS Condition}

Let $\left\{\alpha_{t}\right\}_{t \in \mathbb{R}} \subseteq$ Aut $\mathfrak{A}$ be a group of *-automorphisms on a $\mathrm{C}^{*}$-algebra $\mathfrak{A}$ and $\mathrm{F} \subseteq \mathfrak{A}^{*}$ a separating subspace. An element $x \in \mathfrak{A}$ is called analytic for $\left\{\alpha_{t}\right\}_{t \in \mathbb{R}}$ provided there exists a strip $I_{\lambda, x}=\{z \in \mathbb{C}:|\operatorname{Im} z|<\lambda\}, \lambda>0$, about the real axis and a function $f_{x}: I_{\lambda, x} \longrightarrow \mathfrak{A}$ such that $f_{x}(t)=\alpha_{t}(x)$ for all $t \in \mathbb{R}$ and such that $I_{\lambda, x} \ni z \mapsto\left\langle f_{x}(z), \varphi\right\rangle$ is holomorphic for all $\varphi \in \mathrm{F}$. Then we write $\sigma_{z}(x)=f_{x}(z), z \in I_{\lambda, x}$. If $z \mapsto\left\langle\alpha_{t}(x), \varphi\right\rangle$ is entire for all $\varphi \in \mathrm{F}$ then $x$ is called entire analytic. The set of all entire analytic elements is denoted by $\mathfrak{A}_{\mathrm{e}}$. It can be shown [6] that if $\left\{\alpha_{t}\right\}_{t \in \mathbb{R}}$ is strongly continuous and $\mathrm{F}=\mathfrak{A}^{*}$ then $\mathfrak{A}_{\mathrm{e}}$ is dense in $\mathfrak{A}$. Moreover, if $\left\{\alpha_{t}\right\}_{t \in \mathbb{R}}$ is an ultraweakly continuous group on a von Neumann algebra $\mathfrak{M}$ and if $\mathrm{F}=\mathfrak{M}_{*}$ then $\mathfrak{M}_{\mathrm{e}}$ is ultraweakly dense in $\mathfrak{M}$. In particular, if $\omega(x)=\left\langle\xi_{0}, x \xi_{0}\right\rangle$, where $\xi_{0} \in \mathscr{H}$ is a cyclic and separating vector, we have for the modular group $\sigma_{t}^{\omega}(x)=\Delta^{\mathrm{i} t} x \Delta^{-\mathrm{i} t}$. If $x \in \mathfrak{M}_{\mathrm{e}}$ then $\sigma_{z}^{\omega}(x)=\Delta^{\mathrm{i} z} x \Delta^{-\mathrm{i} z} \in \mathfrak{M}$ for any $z \in \mathbb{C}$. This equalty follows from $\left\langle\eta, \sigma_{z}^{\omega}(x) \xi\right\rangle=\left\langle\Delta^{-\mathrm{i} z} \eta, x \Delta^{-\mathrm{i} z} \xi\right\rangle$ for all $\left.\left.\eta, \xi \in\left\{P_{\Delta}(] a, b\right]\right) \xi: a<b, \xi \in \mathscr{H}\right\}$, where $P_{\Delta}$ denotes the spectral measure of $\Delta$, since these vectors are analytic for $\Delta$ and since an entire function is determined by its values on the real axis. Finally we remark that since weak analyticity implies strong analyticity it follows that $\mathfrak{M}_{\mathrm{e}}$ is an ultraweakly dense *-subalgebra of $\mathfrak{M}$.

Let $\left\{\alpha_{t}\right\}_{t \in \mathbb{R}}$ be a strongly continuous group of $*_{\text {-automorphisms on a }}$ $\mathrm{C}^{*}$-algebra $\mathfrak{A}$. A state $\omega$ on $\mathfrak{A}$ is called a $\left(\left\{\alpha_{t}\right\}_{t \in \mathbb{R}}, \beta\right)$-KMS state, $\beta \in \mathbb{R}$, if

$$
\omega\left(x \alpha_{\mathrm{i} \beta}(y)\right)=\omega(y x)
$$

for all $x, y \in \mathfrak{A}_{\mathrm{e}}$. Similarly, if $\left\{\alpha_{t}\right\}_{t \in \mathbb{R}}$ is an ultraweakly continuous group of *-automorphisms on a von Neumann algebra $\mathfrak{M}$ then a normal state $\omega$ on $\mathfrak{M}$ is called a $\left(\left\{\alpha_{t}\right\}_{t \in \mathbb{R}}, \beta\right)-K M S$ state if $(2.5)$ holds for all $x, y \in \mathfrak{M}_{\mathrm{e}}$.

It can be shown that if $\omega$ is a $\left(\left\{\alpha_{t}\right\}_{t \in \mathbb{R}}, \beta\right)$-KMS state on a $\mathrm{C}^{*}$-algebra $\mathfrak{A}$ and if $\left(\pi_{\omega}, \mathscr{H}_{\omega}, \xi_{\omega}\right)$ is the corresponding GNS representation then it follows that $\xi_{\omega}$ is separating for $\mathfrak{M}=\pi_{\omega}(\mathfrak{A})^{\prime \prime}$, hence $\hat{\omega}(x)=\left\langle\xi_{\omega}, x \xi_{\omega}\right\rangle$ is faithful on $\mathfrak{M}$. As has already been mentioned in Chapter 1 there is a close 
connection between KMS states and the modular group: A state $\omega$ on a $\mathrm{C}^{*}$-algebra $\mathfrak{A}$ is a $\left(\left\{\alpha_{t}\right\}_{t \in \mathbb{R}},-1\right)$-KMS state for a group $\left\{\alpha_{t}\right\}_{t \in \mathbb{R}}$ on $\mathfrak{M}=$ $\pi_{\omega}(\mathfrak{A})^{\prime \prime}$ if and only if $\hat{\omega}$ is faithful; in this case the group $\left\{\alpha_{t}\right\}_{t \in \mathbb{R}}$ is uniquely determined by the KMS condition and coincides with the modular group corresponding to $\hat{\omega}$.

\subsection{Asymptotic Splittings}

In this section we will establish the basic tools which enable us to prove that a system with Markovian time evolution displays decoherence.

\subsubsection{Isometric-Sweeping Splitting}

Let $\mathfrak{M}$ be a von Neumann algebra acting on a Hilbert space $\mathscr{H}$. The main goal of this section is to prove the following theorem.

Theorem 2.3 Let $\left\{T_{t}\right\}_{t \geq 0}$ be an ultraweakly continuous semigroup and $\omega$ a faithful normal state on $\mathfrak{M}$ such that following conditions are satisfied:

1. The state $\omega$ is subinvariant, $\omega \circ T_{t} \leq \omega$ for all $t \geq 0$.

2. Each $T_{t}, t \geq 0$, is strongly positive and unital.

3. Let $\left\{\sigma_{t}^{\omega}\right\}_{t \in \mathbb{R}}$ denote the modular group corresponding to the state $\omega$. Then $\left[T_{t}, \sigma_{s}^{\omega}\right]=0$ for all $s \in \mathbb{R}$ and $t \geq 0$.

Then there exists a von Neumann subalgebra $\mathfrak{M}_{1}$ of $\mathfrak{M}$ and a ${ }^{*}$-invariant ultraweakly closed subspace $\mathfrak{M}_{2}$ of $\mathfrak{M}$ such that ${ }^{1}$

$$
\mathfrak{M}=\mathfrak{M}_{1} \oplus \mathfrak{M}_{2} .
$$

Both $\mathfrak{M}_{1}$ and $\mathfrak{M}_{2}$ are $T_{t}$-invariant for all $t \geq 0$. There exists a group of *-automorphisms $\left\{\alpha_{t}\right\}_{t \in \mathbb{R}} \subseteq$ Aut $\mathfrak{M}_{1}$ and $\left\{T_{t}\right\}_{t \geq 0}$, when restricted to $\mathfrak{M}_{1}$, is given by $\left\{\alpha_{t}\right\}_{t \in \mathbb{R}}$, i. e. $T_{t}\left\lceil\mathfrak{M}_{1}=\alpha_{t}\right.$ for all $t \geq 0$. Moreover, the subalgebra $\mathfrak{M}_{1}$ is the largest $T_{t}$-invariant von Neumann subalgebra of $\mathfrak{M}$ on which the restriction $\left\{T_{t}\right\}_{t \geq 0}$ is given by a group of *automorphisms. The subspace $\mathfrak{M}_{2}$ has the property

$$
\lim _{t \rightarrow \infty} T_{t}(x)=0 \quad \text { for all } x \in \mathfrak{M}_{2}
$$

in the ultraweak topology. Finally, there exists a normal conditional expectation $E$ onto $\mathfrak{M}_{1}$ such that $\left[T_{t}, E\right]=0$ for all $t \geq 0$ and $\omega \circ E=\omega$.

${ }^{1}$ Here we mean the algebraic direct sum, i. e. $\mathfrak{M}=\mathfrak{M}_{1}+\mathfrak{M}_{2}$ and $\mathfrak{M}_{1} \cap \mathfrak{M}_{2}=\{0\}$. 
The splitting (2.6) is called the isometric-sweeping splitting. It was first introduced in [105] in the case $\mathfrak{M}=\mathrm{L}(\mathscr{H})$. In [94] the existence of the isometric-sweeping splitting was established in the more general situation that $\omega$ is a faithful normal semifinite weight which is subinvariant under $\left\{T_{t}\right\}_{t \geq 0}$; then another technical assumption is necessary. We give a simpler proof which works by a completely different method than the one presented in [94].

The proof of Theorem 2.3 will use two lemmas that we establish now. The first is a result from [114]. It explicitly defines the subalgebra $\mathfrak{M}_{1}$ of Theorem 2.3.

Lemma 2.4 Assume that conditions 1. and 2. of Theorem 2.3 are satisfied. Introduce the subsets

$$
\begin{gathered}
M=\left\{x \in \mathfrak{M}: T_{t}\left(x^{*} x\right)=T_{t}(x)^{*} T_{t}(x) \text { for all } t \geq 0\right\}, \\
M^{*}=\left\{x \in \mathfrak{M}: T_{t}\left(x x^{*}\right)=T_{t}(x) T_{t}(x)^{*} \text { for all } t \geq 0\right\}, \\
\mathfrak{M}_{1}=M \cap M^{*} .
\end{gathered}
$$

Then $\mathfrak{M}_{1}$ is a $T_{t}$-invariant von Neumann subalgebra of $\mathfrak{M}$. Moreover, there exists a group of ${ }^{*}$-automorphisms $\left\{\alpha_{t}\right\}_{t \in \mathbb{R}} \subseteq$ Aut $\mathfrak{M}_{1}$ with the property that $T_{t}\left\lceil\alpha_{t}\right.$ for all $t \geq 0$.

Proof. Let $x \in M$ and $s, t \geq 0$, then by the semigroup property and by Kadison's inequality we obtain

$$
\begin{aligned}
T_{s+t}\left(x^{*} x\right) & =T_{s}\left(T_{t}(x)^{*} T_{t}(x)\right)=T_{s}\left(T_{t}(x)\right)^{*} T_{s}\left(T_{t}(x)\right) \\
& \leq T_{s}\left(T_{t}(x)^{*} T_{t}(x)\right) \\
& =T_{s}\left(T_{t}\left(x^{*} x\right)\right) \\
& =T_{s+t}\left(x^{*} x\right)
\end{aligned}
$$

thus $T_{t}(x) \in M$ and $T_{t}(M) \subseteq M$ for any $t \geq 0$. Similarly we conclude that $T_{t}\left(M^{*}\right) \subseteq M^{*}$, and hence $T_{t}\left(\mathfrak{M}_{1}\right) \subseteq \mathfrak{M}_{1}$ for any $t \geq 0$. By an analogous calculationw e see that $\mathfrak{M}_{1} \subseteq T_{t}\left(\mathfrak{M}_{1}\right)$, thus $T_{t}\left(\mathfrak{M}_{1}\right)=\mathfrak{M}_{1}$ for any $t \geq 0$. Clearly $\mathfrak{M}_{1}$ is *-invariant. As in Lemma 1.17 , define for each $t \geq 0$ a sesquilinear form $D_{t}: \mathfrak{M} \times \mathfrak{M} \longrightarrow \mathbb{C}$ by $D_{t}(x, y)=$ $\omega\left(T_{t}\left(x^{*} y\right)-T_{t}(x)^{*} T_{t}(y)\right)$. Then by Kadison's inequality $D_{t}$ is positive, hence by the Cauchy-Schwarz inequality $D_{t}(x, x)=0$ is equivalent to $D_{t}(x, y)=D_{t}(y, x)=0$ for all $y \in \mathfrak{M}$, which in turn, by faithfulness of $\omega$ and Kadison's inequality, implies that

$$
M=\left\{x \in \mathfrak{M}: T_{t}(x y)=T_{t}(x) T_{t}(y) \text { for all } y \in \mathfrak{M}, t \geq 0\right\}
$$


In particular, $M$ and $M^{*}$, and hence $\mathfrak{M}_{1}$, are subspaces. Let $x, y \in M$ and $z \in \mathfrak{M}$, then

$$
T_{t}(x y z)=T_{t}(x) T_{t}(y z)=T_{t}(x) T_{t}(y) T_{t}(y)=T_{t}(x y) T_{t}(z),
$$

hence $x y \in M$, consequently $M$ as well as $M^{*}$ are closed under multiplication, therefore $\mathfrak{M}_{1}$ is a ${ }^{*}$-subalgebra. Let $\left\{x_{i}\right\}_{i \in I} \subseteq M$ be a net with $\lim x_{i}=x$. Then by ultraweak continuity of $x \mapsto a x$ and $x \mapsto x^{*}$ we obtain $0=\lim D_{t}\left(x_{i}, y\right)=D_{t}(x, y)$ for any $y \in \mathfrak{M}$, hence $M$ and also $\mathfrak{M}_{1}$ are ultraweakly closed and we have established that $\mathfrak{M}_{1}$ is a von Neumann subalgebra.

Define $\alpha_{t}=T_{t}\left\lceil\mathfrak{M}_{1}\right.$ for $t \geq 0$. Then by (2.10) $\alpha_{t}$ is a ${ }^{*}$-homomorphism of $\mathfrak{M}_{1}$ into $\mathfrak{M}_{1}$. It follows from assumption 1 . and Corollary 1.32 that $\omega$ is invariant under $\left\{T_{t}\right\}_{t \geq 0}$ and hence under $\alpha_{t}$, thus $\omega\left(x^{*} x\right)=\omega\left(\alpha_{t}\left(x^{*} x\right)\right)=$ $\omega\left(\alpha_{t}(x)^{*} \alpha_{t}(x)\right)$ implies that $\alpha_{t}$ is faithful. Now if $\alpha_{t}(x)=0$ for an arbitrary $x \in \mathfrak{M}_{1}$ then $0=\alpha_{t}(x)^{*} \alpha_{t}(x)=\alpha_{t}\left(x^{*} x\right)$, hence $x=0$ and $\alpha_{t}$ is injective. Surjectivity follows from $T_{t}\left(\mathfrak{M}_{1}\right)=\mathfrak{M}_{1}$, and the proof is finished.

For later use we record the following.

Corollary 2.5 Under the assumptions of Lemma 2.4 we have the following characterizations of $\mathfrak{M}_{1}$ :

$$
\begin{aligned}
M & =\left\{x \in \mathfrak{M}: T_{t}(x y)=T_{t}(x) T_{t}(y) \text { for all } y \in \mathfrak{M}, t \geq 0\right\} \\
& =\left\{x \in \mathfrak{M}: \omega\left(x^{*} x-T_{t}(x) T_{t}(x)^{*}\right) \text { for all } t \geq 0\right\}, \\
M^{*} & =\left\{x \in \mathfrak{M}: T_{t}(y x)=T_{t}(y) T_{t}(x) \text { for all } y \in \mathfrak{M}, t \geq 0\right\} \\
& =\left\{x \in \mathfrak{M}: \omega\left(x x^{*}-T_{t}(x)^{*} T_{t}(x)\right) \text { for all } t \geq 0\right\} .
\end{aligned}
$$

Proof. This follows from the previous proof.

The next lemma uses a technique from [71].

Lemma 2.6 Suppose that conditions 1. and 2. of Theorem 2.3 are satisfied. Then for all $x \in \mathfrak{M}$ any ultraweak limit point of the net $\left\{T_{t}(x)\right\}_{t \in \mathbb{R}^{+}}$ lies in $\mathfrak{M}_{1}$, where $\mathfrak{M}_{1}$ is defined by (2.9).

Proof. We continue to use the notation of Lemma 2.4. We assume (without loss of generality) that $\omega$ is a vector state given by the cyclic and separating vector $\xi_{0} \in \mathscr{H}$. By strong positivity and invariance of $\omega$ under each $T_{t}$ (Corollary 1.32) we can define the strongly continuous contraction semigroup $\left\{\hat{T}_{t}\right\}_{t \geq 0}$ on $\mathscr{H}$ defined by $\hat{T}_{t}\left(x \xi_{0}\right)=T_{t}(x) \xi_{0}$ for all $x \in \mathfrak{M}$ and $t \geq 0$. For $x, y \in \mathfrak{M}$ we have

$$
\lim _{t \rightarrow \infty} D_{t}(x, y)=\lim _{t \rightarrow \infty}\left\langle\xi_{0},\left(T_{t}\left(x^{*} y\right)-T_{t}(x)^{*} T_{t}(y)\right) \xi_{0}\right\rangle
$$




$$
\begin{aligned}
& =\lim _{t \rightarrow \infty}\left(\left\langle\xi_{0}, x^{*} y \xi_{0}\right\rangle-\left\langle\hat{T}_{t}\left(x \xi_{0}\right), \hat{T}_{t}\left(y \xi_{0}\right)\right\rangle\right) \\
& =\lim _{t \rightarrow \infty}\left\langle\xi_{0}, x^{*}\left(\mathbb{1}-\hat{T}_{t}^{*} \hat{T}_{t}\right) y \xi_{0}\right\rangle \\
& =\left\langle\xi_{0}, x^{*}(\mathbb{1}-\hat{T}) y \xi_{0}\right\rangle
\end{aligned}
$$

where $\hat{T}=\operatorname{s-lim}_{t \rightarrow \infty} \hat{T}_{t}^{*} \hat{T}_{t}$, which exists by [19], p. 41. Furthermore, we have

$$
\begin{aligned}
D_{t}\left(T_{s}(x), T_{s}(x)\right)= & \left\langle\xi_{0}, T_{s}(x)^{*} T_{s}(x) \xi_{0}\right\rangle-\left\langle\xi_{0}, T_{s+t}(x)^{*} T_{s+t}(x) \xi_{0}\right\rangle \\
= & \left\langle\xi_{0},\left(T_{s+t}\left(x^{*} x\right)-T_{s+t}(x)^{*} T_{s+t}(x)\right) \xi_{0}\right\rangle \\
& \quad-\left\langle\xi_{0},\left(T_{t}\left(T_{s}\left(x^{*} x\right)\right)+T_{s}(x)^{*} T_{s}(x)\right) \xi_{0}\right\rangle \\
= & D_{s+t}(x, x)-D_{s}(x, x)
\end{aligned}
$$

and using (2.15) this implies

$$
\lim _{s \rightarrow \infty} D_{t}\left(T_{s}(x), T_{s}(x)\right)=0 \quad \text { for all } x \in \mathfrak{M}, t \geq 0 .
$$

Therefore, by the Cauchy-Schwarz inequality we obtain

$$
\left|D_{t}\left(T_{s}(x), y\right)\right| \leq D_{s}\left(T_{s}(x), T_{s}(x)\right) D_{t}(y, y) \rightarrow 0
$$

as $s \rightarrow \infty$ for all $y \in \mathfrak{M}$ and $t \geq 0$. Now let $x_{0} \in \mathfrak{M}$ be an ultraweak limit point of the net $\left\{T_{t}(x)\right\}_{t \in \mathbb{R}^{+}}$for some $x \in \mathfrak{M}$. Then there exists a subnet $\left\{t_{i}\right\}_{i \in I} \subseteq \mathbb{R}^{+}$such that $\lim _{i \rightarrow \infty} T_{t_{i}}(x)=x_{0}$ ultraweakly. Thus by (2.16) we have

$$
\lim _{i \rightarrow \infty} D_{t}\left(T_{t_{i}}(x), y\right)=D_{t}\left(x_{0}, y\right)=0
$$

for all $y \in \mathfrak{M}$ and $t \geq 0$ using continuity of $x \mapsto a x$ and $x \mapsto x^{*}$, and normality of $T_{t}$. In particular, $D_{t}\left(x_{0}, x_{0}\right)=0$ for all $t \geq 0$, and similarly $D_{t}\left(x_{0}^{*}, x_{0}^{*}\right)=0$, and we conclude that $x_{0} \in \mathfrak{M}_{1}$, as was to be shown.

Proof. (Proof of Theorem 2.3.) Clearly, $\mathfrak{M}_{1}$, as defined by (2.9), is the largest von Neumann subalgebra on which $\left\{T_{t}\right\}_{t \geq 0}$, when restricted, is given by a group of *-automorphisms.

Let $x \in \mathfrak{M}_{1}$ and $s \in \mathbb{R}$ and $t \geq 0$. Then by assumption 3 . we have

$$
\begin{aligned}
T_{t}\left(\sigma_{s}^{\omega}(x)^{*} \sigma_{s}^{\omega}(x)\right) & =T_{t}\left(\sigma_{s}^{\omega}\left(x^{*} x\right)\right) \\
& =\sigma_{s}^{\omega}\left(T_{t}\left(x^{*} x\right)\right) \\
& =\sigma_{s}^{\omega}\left(T_{t}(x)^{*} T_{t}(x)\right) \\
& =\sigma_{s}^{\omega}\left(T_{t}(x)\right)^{*} \sigma_{s}^{\omega}\left(T_{t}(x)\right),
\end{aligned}
$$


thus $\sigma_{s}^{\omega}\left(\mathfrak{M}_{1}\right) \subseteq \mathfrak{M}_{1}$ and by Theorem 2.2 there exists a normal conditional expectation $E: \mathfrak{M} \longrightarrow \mathfrak{M}$ with $\operatorname{ran} E=\mathfrak{M}_{1}$ and $\omega \circ E=\omega$. Define $E^{\perp}=\mathbb{1}-E$ and $\mathfrak{M}_{2}=\operatorname{ran} E^{\perp}$. Since $E^{\perp}\left(x^{*}\right)=E^{\perp}(x)^{*}, \mathfrak{M}_{2}$ is a ${ }_{-}$ invariant ultraweakly closed subspace and (2.6) holds. Introduce on $\mathfrak{M}$ the GNS inner product $\langle x, y\rangle=\omega\left(x^{*} y\right)$ and write $\|x\|_{0}^{2}=\langle x, x\rangle$ for the corresponding norm. By $\omega$-invariance of $E$ and by the properties of conditional expectation we have

$$
\begin{aligned}
\langle x, E(y)\rangle & =\omega\left(x^{*} E(y)\right)=\omega\left[E\left(x^{*} E(y)\right)\right] \\
& =\omega\left[E\left(x^{*}\right) E(y)\right]=\omega\left[E\left(E(x)^{*} y\right)\right], \\
& =\langle E(x), y\rangle
\end{aligned}
$$

that is $E=E^{\prime}=E^{2}$ where the prime denotes adjoints with respect to $\langle\cdot, \cdot\rangle$. We now prove that

$$
\mathfrak{M}_{1}^{\perp}:=\left\{x \in \mathfrak{M}:\langle x, y\rangle=0 \text { for all } y \in \mathfrak{M}_{1}\right\}=\mathfrak{M}_{2}=\operatorname{ran} E^{\perp} .
$$

Let $x \in \mathfrak{M}_{2}$, then $x=E^{\perp}(z)$ for some $z \in \mathfrak{M}$ and $\langle x, y\rangle=\left\langle E^{\perp}(z), y\right\rangle=$ $\left\langle z, E^{\perp}(y)\right\rangle=0$ for all $y \in \mathfrak{M}_{1}$ hence $x \in \mathfrak{M}_{1}^{\perp}$. Conversely, let $x \in \mathfrak{M}_{1}^{\perp}$, then $\langle x, E(z)\rangle=0$ for all $z \in \mathfrak{M}$. This implies $\langle x, z\rangle=\langle x, z\rangle-\langle x, E(z)\rangle=$ $\left\langle x, E^{\perp}(z)\right\rangle=\left\langle E^{\perp}(x), z\right\rangle$, hence $E^{\perp}(x)=x$ and thus $x \in \mathfrak{M}_{2}$. Thus we have established $\mathfrak{M}_{2}=\mathfrak{M}_{1}^{\perp}$.

Next we note that in view of Kadison's inequality and invariance of $\omega$, $T_{t}$ is a contraction in $\|\cdot\|_{0}:\left\|T_{t}(x)\right\|_{0}^{2} \leq \omega\left(T_{t}\left(x^{*} x\right)\right)=\|x\|_{0}^{2}$. Now pass to the completion of the pre-Hilbert space $(\mathfrak{M},\langle\cdot, \cdot\rangle)$ and consider the adjoint $T_{t}^{\prime}$ of $T_{t}$ on this Hilbert space; then $T_{t}^{\prime}$ is a contraction as well. If $\left\|T_{t}(x)\right\|_{0}=\|x\|_{0}$ for some $x \in \mathfrak{M}$ and $t \geq 0$ then

$$
\begin{aligned}
\left\|T_{t}^{\prime}\left(T_{t}(x)\right)-x\right\|_{0}^{2} & =\left\|T_{t}^{\prime}\left(T_{t}(x)\right)\right\|_{0}^{2}-2 \operatorname{Re}\left\langle T_{t}^{\prime}\left(T_{t}(x)\right), x\right\rangle+\|x\|_{0}^{2} \\
& \leq 2\|x\|_{0}^{2}-2\left\|T_{t}(x)\right\|_{0}^{2} \\
& =0
\end{aligned}
$$

i. e. $T_{t}^{\prime}\left(T_{t}(x)\right)=x$. In particular, $T_{t}^{\prime}\left(T_{t}(x)\right)=x \in \mathfrak{M}_{1}$ for all $x \in \mathfrak{M}_{1}$. Since $T_{t}$ is bijective on $\mathfrak{M}_{1}$ this implies that $T_{t}^{\prime}\left(\mathfrak{M}_{1}\right) \subseteq \mathfrak{M}_{1}$ for all $t \geq 0$. Now if $x \in \mathfrak{M}_{2}$ this result, together with what was shown above, implies

$$
\left\langle T_{t}(x), y\right\rangle=\left\langle x, T_{t}^{\prime}(y)\right\rangle=0 \quad \text { for all } y \in \mathfrak{M}_{1},
$$

and $T_{t}(x) \in \mathfrak{M}_{1}^{\perp}=\mathfrak{M}_{2}$. Thus we have shown that $T_{t}\left(\mathfrak{M}_{2}\right) \subseteq \mathfrak{M}_{2}$ for all $t \geq 0$, i. e. both $\mathfrak{M}_{1}$ and $\mathfrak{M}_{2}$ are $T_{t}$-invariant. Since $x=E(x)+E^{\perp}(x)$ we conclude from $T_{t}(x)=T_{t}(E(x))+T_{t}\left(E^{\perp}(x)\right)$ that $T_{t} \circ E=E \circ T_{t}$ for all $t \geq 0$. 
Only property (2.7) remains to be shown. Let $x \in \mathfrak{M}_{2}$ and suppose that $\|x\| \leq 1$. Then by ultraweak compactness the net $\left\{T_{t}(x)\right\}_{t \in \mathbb{R}^{+}} \subseteq$ ball $\mathfrak{M}_{2}$ has an ultraweak limit point $x_{0}$, and $x_{0} \in \mathfrak{M}_{2}$ by closedness of $\mathfrak{M}_{2}$. But in view of Lemma 2.6 we have $x_{0} \in \mathfrak{M}_{1}$, hence $x \in \mathfrak{M}_{1} \cap \mathfrak{M}_{2}=\{0\}$. This shows that any ultraweak limit point of the net $\left\{T_{t}(x)\right\}_{t \in \mathbb{R}^{+}}$is equal to 0 , hence we have $(2.7)$.

\subsubsection{Jacobs-de Leeuw-Glicksberg Splitting}

In the previous section we introduced the isometric-sweeping splitting (Theorem 2.3) for a quantum dynamical semigroup on a von Neumann algebra. In the present subsection we discuss another asymptotic splitting, the Jacobs-de Leeuw-Glicksberg splitting, which is closely related to the isometric-sweeping splitting. In fact, we shall provide an example which shows that it is of potential interest in the discussion of environmental decoherence and can provide information in situations in which the assumptions of Theorem 2.3 cannot be verified. Originally it was introduced for weakly almost periodic semigroups $[93,20]$, here we apply it to weak* almost periodic quantum dynamical semigroups on von Neumann algebras and give a proof for that case.

\section{Banach Space Case}

We shall prove the existence of the Jacobs-de Leeuw-Glicksberg splitting first in the more general case of a weak* continuous semigroup on a Banach space $X$ having a predual space $X_{*}$. In Section 1.4 .2 it was shown that the pointwise weak* topology on $\mathrm{L}(\mathrm{X})$ agrees with the $\sigma\left(\mathrm{L}(\mathbf{X}), \mathbf{X} \otimes_{\gamma} \mathbf{X}_{*}\right)$ topology; consequently, by Alaoglu's theorem ball $\mathrm{L}(\mathrm{X})$ is compact in this topology. Let $\left\{T_{t}\right\}_{t \geq 0}$ be a weak* continuous semigroup of contractive operators on $\mathrm{X}$. We can consider $\mathrm{L}(\mathrm{X})$ as a semigroup under multiplication and $\mathrm{S}_{0}=\left\{T_{t}: t \geq 0\right\}$ as a commutative subsemigroup consisting of weak*-weak* continuous operators. Let $\mathrm{S}$ denote the closure of $\mathrm{S}_{0}$ in the pointwise weak* topology. In the following it is important that $\mathrm{S}$ also consists of weak ${ }^{*}-$ weak $^{*}$ continuous operators so we provide some sufficient conditions in the following proposition.

Proposition 2.7 Let $\mathrm{S}_{0}$ and $\mathrm{S}$ be as above, and consider the following conditions:

1. Both $\mathbf{X}$ and $\mathbf{X}_{*}$ are separable and $\mathbf{X}_{*}$ is weakly sequentially complete.

2. Both $\mathrm{X}$ and $\mathrm{X}_{*}$ are separable and $\mathrm{X}_{*}$ is reflexive. 
3. The restriction of the semigroup $\left\{T_{t}\right\}_{t \geq 0}$ to ball $\mathrm{X}$ is weak ${ }^{*}$ equicontinuous.

Under either of the above conditions $\mathrm{S}$ consists of weak ${ }^{*}-w e a k^{*}$ continuous operators.

Proof. Assume that condition 1. holds. Let $\left\{x_{n}\right\}_{n \in \mathbb{N}} \subseteq \mathrm{X}$ and $\left\{x_{*, n}\right\}_{n \in \mathbb{N}} \subseteq$ $\mathrm{X}_{*}$ be dense. Then the pointwise weak* topology is induced by the countable family of seminorms $\left\{p_{x_{m}, x_{*}, n}: m, n \in \mathbb{N}\right\}$, where $p_{x, x_{*}}(T)=$ $\left\langle T(x), x_{*}\right\rangle, T \in \mathrm{L}(\mathrm{X})$, and hence is metrizable on ball $\mathrm{L}(\mathrm{X})$. Given any $T \in \mathrm{S}$ there is a sequence $\left\{T_{k}\right\}_{k \in \mathbb{N}} \subseteq \mathrm{S}_{0}$ with $T_{k} \rightarrow T$ relative to the pointwise weak ${ }^{*}$ topology. It follows that for each $x_{*} \in \mathrm{X}_{*}$ the functionals $f_{k}(x)=\left\langle T_{k}(x), x_{*}\right\rangle$ are in $\mathrm{X}_{*}$ and converge weakly to the functional $x \mapsto\left\langle T(x), x_{*}\right\rangle$. By weak sequential completeness this functional is weak ${ }^{*}$ continuous, hence $T$ is weak ${ }^{*}-$ weak $^{*}$ continuous.

Assume that condition 2. holds. Then $\mathbf{X}_{*}=\mathbf{X}^{*}$, and the $\sigma\left(\mathbf{X}_{*}, \mathbf{X}\right)$ topology agrees with the $\sigma\left(\mathbf{X}^{*}, \mathbf{X}\right)$ topology on $\mathbf{X}_{*}$. The assertion follows from 1 . and weak*-sequential completeness.

Assume that condition 3. holds and let $T \in \mathrm{S}$. Then there is a net $\left\{T_{i}\right\}_{i \in I} \subseteq \mathrm{S}_{0}$ such that $T_{i} \rightarrow T$ relative to the pointwise weak ${ }^{*}$ topology. Now let $\left\{x_{j}\right\}_{J \in I} \subseteq$ ball $\mathrm{X}$ with $x_{i} \rightarrow x$ relative to the weak* topology. For $\epsilon>0$ and $x_{*} \in \mathrm{X}_{*}$ there exists, by assumption, a $j_{0} \in J$ such that $\left|\left\langle T_{i}\left(x_{j}-x\right), x_{*}\right\rangle\right| \leq \epsilon$ if $j \geq j_{0}$ uniformly for $i \in I$. Letting $i \rightarrow \infty$ this implies $\left|\left\langle T\left(x_{j}-x\right), x_{*}\right\rangle\right| \leq \epsilon$ if $j \geq j_{0}$. This proves that $T$ is weak ${ }^{*}{ }_{\text {-weak }}{ }^{*}$ continuous when restricted to ballX. By an application of the KreinŠmulian theorem we conclude that $T$ is weak ${ }^{*}{ }_{-}$weak $^{*}$ continuous.

Assume now that S consists of weak ${ }^{*}{ }^{-}$weak $^{*}$ continuous operators. The commutative semigroup $\mathrm{S}$ is semitopological under the relative pointwise weak* $^{*}$ topology on $\mathrm{S}$, i. e. the maps $T \mapsto T Q, T \mapsto Q T$ are pointwise weak* continuous for every $Q \in S$ since $S$ consists of weak ${ }^{*}$-weak ${ }^{*}$ continuous operators. This means that we have shown that $\mathrm{S}$ is a commutative compact semitopological semigroup consisting of weak ${ }^{*}$-weak ${ }^{*}$ continuous operators. We now use the fact that every commutative compact semitopological semigroup $\mathrm{S}$ contains a unique minimal ideal $\mathrm{G} \subseteq \mathrm{S}$ that is a compact group which is given by

$$
\mathrm{G}=\bigcap_{R \in \mathrm{S}} R \mathrm{~S},
$$

and if $Q \in \mathrm{G}$ denotes the unit of $\mathrm{G}$ then $\mathrm{G}=Q \mathrm{~S}$. This ideal $\mathrm{G}$ is called the Sushkevich kernel of S. By compactness and Ellis' theorem the semitopological group $\mathrm{G}$ is a topological group. 
We are now able to prove the following version of the Jacobs-de LeeuwGlicksberg splitting theorem (cf. [11, 93, 20]). The proof mimicks the one given in [11] for weakly almost periodic one-parameter semigroups.

Theorem 2.8 Let $\mathrm{S}_{0}=\left\{T_{t}\right\}_{t \geq 0}$ be a weak* continuous contractive semigroup on the Banach space $\mathbf{X}$ with predual $\mathrm{X}_{*}$ and let $Z$ be its weak* generator. Assume that $\mathrm{S}=\overline{\mathrm{S}}_{0}$ consists of weak ${ }^{*}-$ weak ${ }^{*}$ continuous operators. Then there exist weak ${ }^{*}$-closed subspaces $\mathbf{X}_{\mathbf{s}}, \mathbf{X}_{\mathrm{r}}$ of $\mathbf{X}$ invariant under $T_{t}$, $t \geq 0$, such that $\mathbf{X}=\mathbf{X}_{\mathrm{s}} \oplus \mathbf{X}_{\mathrm{r}}$, and

$$
\begin{aligned}
\mathrm{X}_{\mathrm{s}} & =\left\{x \in \mathrm{X}: 0 \in{\overline{\left\{T_{t}(x): t \geq 0\right\}}}^{\mathrm{w}^{*}}\right\}, \\
\mathrm{X}_{\mathrm{r}} & =\overline{\operatorname{lin}\{x \in \operatorname{dom} Z: \exists \alpha \in \mathbb{R} \text { such that } Z x=\mathrm{i} \alpha x\}}{ }^{\mathrm{w}^{*}} \\
& =\varlimsup \operatorname{lin}\left\{x \in \mathrm{X}: \exists \alpha \in \mathbb{R} \text { such that } T_{t}(x)=\mathrm{e}^{\mathrm{i} \alpha t} x \forall t \geq 0\right\}
\end{aligned}
$$

Proof. Let $Q$ be the unit of the Sushkevich kernel $\mathrm{G} \subseteq \mathrm{S}$. Then $Q^{2}=Q$, i. e. $Q$ is a weak*-weak* continuous projection on $\mathrm{X}$ satisfying $\left[Q, T_{t}\right]=0$ for all $t \geq 0$. The theorem will be established once we prove that $\mathrm{X}_{\mathrm{s}}=$ $\operatorname{ker} Q$ and $\mathrm{X}_{\mathrm{r}}=\operatorname{ran} Q$, where $\mathbf{X}_{\mathbf{s}}$ and $\mathbf{X}_{\mathbf{s}}$ are defined by (2.18) and (2.19), respectively.

Let $x \in \operatorname{ker} Q$. Since $Q \in \mathrm{S}$ there is a net $\left\{T_{i}\right\}_{i \in I} \subseteq \mathrm{S}_{0}$ such that $T_{i} \rightarrow Q$ relative to the pointwise weak ${ }^{*}$ topology, hence $T_{i}(x) \rightarrow Q x=0$ relative to the weak*-topology, thus $0 \in \overline{\left\{T_{t}(x): t \geq 0\right\}}{ }^{\mathrm{w}^{*}}$. Conversely, let $x \in \mathrm{X}$ and assume that $0 \in{\overline{\left\{T_{t}(x): t \geq 0\right\}}}^{\mathrm{w}^{*}}$. Then there is a net $\left\{T_{i}\right\}_{i \in I} \subseteq \mathrm{S}_{0}$ such that $T_{i}(x) \rightarrow 0$ relative to the weak*-topology. By compactness of $\mathrm{S}$ there is a subnet $\left\{T_{j}\right\}_{j \in J} \subseteq\left\{T_{i}\right\}_{i \in I}$ with $T_{j} \rightarrow R$ relative to the pointwise weak* topology for some $R \in \mathrm{S}$, and it follows that $R x=0$. Hence $R^{\prime} Q R x=0$ for all $R^{\prime} \in \mathrm{S}$. Choosing $R^{\prime}$ to be the inverse of $Q R$ in $\mathrm{G}$ we get $Q x=0$, hence $x \in \operatorname{ker} Q$. We have thus proved that $\mathrm{X}_{\mathrm{s}}=\operatorname{ker} Q$.

Let $\hat{\mathrm{G}}$ be the character group of $\mathrm{G}$. For each $\chi \in \hat{\mathrm{G}}$ define the operator

$$
\mathrm{X} \ni x \mapsto P_{\chi} x=\int_{\mathrm{G}} \overline{\chi(S)} S x \mathrm{~d} \mu(S),
$$

where $\mu$ is the normalized Haar measure of G. The integral is to be understood as a weak ${ }^{*}$-integral, thus $P_{\chi}$ is a well-defined bounded operator in $\mathrm{L}(\mathrm{X})$ with $\left\|P_{\chi}\right\| \leq 1$. Then for all $R \in \mathrm{G}$ we get

$$
\begin{aligned}
R P_{\chi} x & =\int_{\mathrm{G}} \overline{\chi(S)} R S x \mathrm{~d} \mu(S)=\chi(R) \int_{\mathrm{G}} \overline{\chi(R S)} R S x \mathrm{~d} \mu(S) \\
& =\chi(R) \int_{\mathrm{G}} \overline{\chi(S)} S x \mathrm{~d} \mu(S)=\chi(R) P_{\chi} x,
\end{aligned}
$$


in particular $Q P_{\chi}=P_{\chi}$, therefore $T_{t} P_{\chi}=T_{t} Q P_{\chi}=\chi\left(T_{t} Q\right) P_{\chi}$ for all $t \geq 0$. Since $t \mapsto \chi\left(T_{t} Q\right)$ is continuous and satisfies the functional equation

$$
\chi\left(T_{t} Q\right) \cdot \chi\left(T_{s} Q\right)=\chi\left(T_{t+s} Q\right) \in\{z \in \mathbb{C}:|z|=1\}
$$

for all $t, s \geq 0$, we have $\chi\left(T_{t} Q\right)=\mathrm{e}^{\mathrm{i} \alpha t}$ for some $\alpha \in \mathbb{R}$. Thus $T_{t} P_{\chi}=$ $\mathrm{e}^{\mathrm{i} \alpha t} P_{\chi}$, hence $P_{\chi} \mathrm{X} \subseteq \operatorname{dom} Z$ and $Z P_{\chi}=\mathrm{i} \alpha P_{\chi}$ for all $\chi \in \hat{\mathrm{G}}$. We next define the subspace

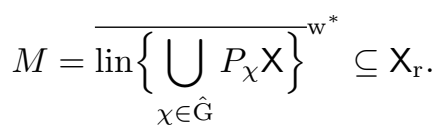

We prove that $\operatorname{ran} Q \subseteq M \subseteq \mathrm{X}_{\mathrm{r}}$. Let $x_{*} \in M^{\perp}=\left\{x_{*} \in \mathrm{X}_{*}:\left\langle x, x_{*}\right\rangle=\right.$ $0 \forall x \in M\}$. Then $\left\langle P_{\chi} x, x_{*}\right\rangle=0$ for all $x \in \mathrm{X}, \chi \in \hat{\mathrm{G}}$, i. e.

$$
\int_{\hat{\mathrm{G}}} \overline{\chi(S)}\left\langle S x, x_{*}\right\rangle \mathrm{d} \mu(S)=0
$$

for all $x \in \mathbf{X}, \chi \in \hat{\mathrm{G}}$. Since the character group $\hat{\mathrm{G}}$ is total in $L^{2}(G, \mu)$ by the Stone-Weierstraß theorem, and since $S \mapsto\left\langle S x, x_{*}\right\rangle$ is pointwise weak* continuous, it follows that $\left\langle S x, x_{*}\right\rangle=0$ for all $S \in \mathrm{G}$ and $x \in \mathrm{X}$. Take $S=Q$, then we obtain $x_{*} \in \operatorname{ran} Q^{\perp}$ and thus $M^{\perp} \subseteq \operatorname{ran} Q^{\perp}$. By the bipolar theorem we obtain $\operatorname{ran} Q \subseteq \overline{\mathrm{co}} M=M$, since $\operatorname{ran} Q$ is a weak* closed subspace. Conversely, let $x \in \operatorname{dom} Z$ with $Z x=\mathrm{i} \alpha x$ for some $\alpha \in \mathbb{R}$. It follows that $T_{t}(x)=\mathrm{e}^{\mathrm{i} \alpha t} x$ for all $t \geq 0$ and consequently $R x=\mathrm{e}^{\mathrm{i} \alpha^{\prime}} x$ for $R \in S$. Thus there exists $\beta \in \mathbb{R}$ such that $Q x=\mathrm{e}^{\mathrm{i} \beta} x=Q^{2} x$, which implies $Q x=x \in \operatorname{ran} Q$, hence $\mathrm{X}_{\mathrm{r}} \subseteq \operatorname{ran} Q$, and proof is finished.

Corollary 2.9 Under the hypothesis of Theorem 2.8, there exists a weak* continuous one-parameter group $\left\{\alpha_{t}\right\}_{t \in \mathbb{R}}$ of isometries on $\mathrm{X}_{\mathrm{r}}$ such that $\alpha_{t}=T_{t} \uparrow \mathrm{X}_{\mathrm{r}}$ for $t \geq 0$.

Proof. Let $T \in \mathrm{S}$, then $Q T \in \mathrm{G}$, and let $R$ be the inverse of $Q T$ in $\mathrm{G}$, i. e. $R(Q T)=Q$. Then for all $x \in \mathrm{X}_{\mathrm{r}}$ we have $R T x=R Q T x=Q x=x$. If $T=T_{t}$ for some $t \geq 0$ define $\alpha_{t}=T_{t} \uparrow \mathrm{X}_{\mathrm{r}}$, and $\alpha_{-t}=R \uparrow \mathrm{X}_{\mathrm{r}}=$ $\left(T_{t}\left\lceil\mathbf{X}_{\mathrm{r}}\right)^{-1}\right.$. It is then clear that $\left\{\alpha_{t}\right\}_{t \in \mathbb{R}}$ is a one-parameter group on $\mathbf{X}_{\mathrm{r}}$. Moreover, it is clear that it is weak* continuous and contractive. Now assume that there is $x \in \mathrm{X}_{\mathrm{r}}$ and $t \geq 0$ such that $\left\|\alpha_{t}(x)\right\|<\|x\|$. Then it follows that $\left\|\alpha_{-t}\right\|>1$, contradiction; thus $\left\{\alpha_{t}\right\}_{t \in \mathbb{R}}$ is isometric.

The subspace $\mathbf{X}_{\mathrm{r}}$ is called the reversible subspace and $\mathbf{X}_{\mathrm{s}}$ is called the stable subspace, its elements are sometimes called flight vectors. 
We now look for a stronger characterization of the stable subspace than (2.18). In particular, we would like to prove that its elements $x$ are characterized by the property $\lim _{t \rightarrow \infty} T_{t}(x)=0$ relative to the weak* topology.

Proposition 2.10 Assume that the hypothesis of Theorem 2.8 is satisfied and additionally that $\operatorname{spec} Z \cap \mathrm{i} \mathbb{R}$ is at most countable. Then the stable subspace (2.18) is given by

$$
\mathbf{X}_{\mathbf{s}}=\left\{x \in \mathbf{X}: \lim _{t \rightarrow \infty} T_{t}(x)=0 \text { relative to the weak* topology }\right\} .
$$

Moreover, the convergence in (2.21) is uniform for $x$ in $\mathbf{X}_{\mathbf{s}} \cap$ ball $\mathrm{X}$.

Proof. The predual operator $Q_{*}$ of $Q$ is a projection on $\mathrm{X}_{*}$, i. e. $Q_{*}^{2}=Q_{*}$. It induces a splitting $\mathrm{X}_{*}=\mathrm{X}_{\mathrm{s}, *} \oplus \mathrm{X}_{\mathrm{r}, *}$ by way of $\mathrm{X}_{\mathrm{s}, *}=\operatorname{ker} Q_{*}$ and $\mathrm{X}_{\mathrm{r}, *}=\operatorname{ran} Q_{*}$. It may be shown that $\mathrm{X}_{\mathrm{s}} \cong\left(\mathrm{X}_{\mathrm{s}, *}\right)^{*}$ and $\mathrm{X}_{\mathrm{r}} \cong\left(\mathrm{X}_{\mathrm{r}, *}\right)^{*}$, and the semigroups $\left\{T_{t, *}\right\}_{t \geq 0}$ and $\left\{T_{t}\right\}_{t \geq 0}$ are compatible with the splitting, i. e. $T_{t, *}=T_{t, *}^{(\mathrm{s})} \oplus T_{t, *}^{(\mathrm{r})}$ and $T_{t}=T_{t}^{(\mathrm{s})} \oplus T_{t}^{(\mathrm{r})}$ in a natural notation. If $Z_{*}$ denotes the generator of the weakly and hence strongly continuous semigroup $\left\{T_{t, *}\right\}_{t \geq 0}$, then the dual $Z=\left(Z_{*}\right)^{*}$ is the generator of the weak* continuous semigroup $\left\{T_{t}\right\}_{t \geq 0}$. If $Z_{\mathrm{s}, *}$ denotes the generator of $\left\{T_{t, *}^{(\mathrm{s})}\right\}_{t \geq 0}$, then $\operatorname{dom} Z_{\mathrm{s}, *}=\operatorname{dom} Z_{*} \cap \mathrm{X}_{\mathrm{s}, *}$ and $Z_{\mathrm{s}, *} x_{*}=Z_{*} x_{*}$ for all $x_{*} \in \operatorname{dom} Z_{\mathrm{s}, *}$, moreover $Z_{\mathrm{s}}=\left(Z_{\mathrm{s}, *}\right)^{*}$.

We prove that $\operatorname{spec} Z_{\mathrm{s}, *} \subseteq \operatorname{spec} Z_{*}$. Let $\lambda \in \rho\left(Z_{*}\right)$, i. e. the map $(\lambda \mathbb{1}-$ $\left.Z_{*}\right): \operatorname{dom} Z_{*} \longrightarrow \mathrm{X}_{*}$ is bijective. Then clearly the map $\left(\lambda \mathbb{1}-Z_{*, \mathrm{~s}}\right)=$ $\left(\lambda \mathbb{1}-Z_{*}\right)\left\lceil\mathrm{X}_{*, \mathrm{~s}}: \operatorname{dom} Z_{*} \cap \mathrm{X}_{\mathrm{s}, *} \longrightarrow \mathrm{X}_{\mathrm{s}, *}\right.$ is injective. It is also surjective: Let $x_{0, *} \in \mathbf{X}_{\mathrm{s}, *}$. Then there exists $x_{*} \in \operatorname{dom} Z_{*}$ such that $\left(\lambda \mathbb{1}-Z_{*}\right) x_{*}=$ $x_{0, *}$. Now

$$
\left(\lambda \mathbb{1}-Z_{*}\right) x_{*}=\left(\lambda \mathbb{1}-Z_{*}\right)\left(x_{1, *} \oplus x_{2, *}\right)=x_{0, *},
$$

i. e. $\left(\lambda \mathbb{1}-Z_{*}\right) x_{1, *}=0$, hence $x_{1, *}=0$ by injectivity and $x_{*} \in \operatorname{dom} Z_{\mathrm{s}, *}$. Thus $\left(\lambda \mathbb{1}-Z_{\mathrm{s}, *}\right)$ is bijective and $\lambda \in \rho\left(Z_{\mathrm{s}, *}\right)$; our claim follows. Using $\operatorname{spec} Z=\operatorname{spec} Z_{*}$, then in particular

$$
\operatorname{spec} Z_{\mathrm{s}, *} \cap \mathrm{i} \mathbb{R} \text { is countable. }
$$

We now prove that

$$
\operatorname{spec}_{\mathrm{p}} Z_{\mathrm{s}} \cap \mathrm{i} \mathbb{R}=\emptyset,
$$

for if $\mathrm{i} \lambda \in \operatorname{spec}_{\mathrm{p}} Z_{\mathrm{s}}, \lambda \in \mathbb{R}$, then the corresponding eigenvector $x \in$ $\operatorname{dom} Z_{\mathrm{s}} \subseteq \mathrm{X}_{\mathrm{s}}$ satisfying $Z x=\mathrm{i} \lambda x$ must lie in $\mathrm{X}_{\mathrm{r}}$ by (2.19), hence $x=0$, contradiction. 
From (2.22) and (2.23) it follows by the Arendt-Batty-Lyubich-Vũ theorem [40, 95], see also [11], that the semigroup $\left\{T_{t, *}^{(\mathrm{s})}\right\}_{t \geq 0}$ is strongly stable, i. e. for all $x_{*} \in \mathrm{X}_{\mathrm{s}, *}$ we have $\lim _{t \rightarrow \infty}\left\|T_{t, *}^{(\mathrm{s})}\left(x_{*}\right)\right\|=0$. Thus if $x \in \mathrm{X}_{\mathrm{s}}$ and $x_{*} \in \mathrm{X}_{*}$ it follows that

$$
\begin{aligned}
\left|\left\langle T_{t}(x), x_{*}\right\rangle\right| & =\left|\left\langle Q\left(T_{t}(x)\right), x_{*}\right\rangle\right|=\left|\left\langle x, T_{t, *}\left(Q_{*}\left(x_{*}\right)\right)\right\rangle\right| \\
& \leq\|x\| \cdot\left\|T_{t, *}^{(\mathrm{s})}\left(Q_{*}\left(x_{*}\right)\right)\right\| \rightarrow 0
\end{aligned}
$$

as $t \rightarrow \infty$ uniformly for $x \in \mathrm{X}_{\mathrm{s}} \cap$ ball $\mathrm{X}$, as was to be shown.

\section{Von Neumann Algebra Case}

The results of the previous subsection apply to the case of ultraweakly continuous semigroups $\left\{T_{t}\right\}_{t \geq 0}$ on a von Neumann algebra $\mathfrak{M}$ by taking $\mathrm{X}=\mathfrak{M}$ and $\mathrm{X}_{*}=\mathfrak{M}_{*}$. We can then state the following.

Corollary 2.11 Let $\mathfrak{M}$ be a von Neumann algebra and $\left\{T_{t}\right\}_{t \geq 0}$ an ultraweakly continuous contractive semigroup on $\mathfrak{M}$, and let $Z$ be its ultraweak generator. Suppose that there exists a faithful family $\Phi$ of normal states on $\mathfrak{M}$ such that

$$
\varphi\left(T_{t}(x)^{*} T_{t}(x)\right) \leq \varphi\left(x^{*} x\right) \quad \text { for all } \varphi \in \Phi, t \geq 0
$$

Then there exist ultraweakly closed $T_{t}$-invariant subspaces $\mathfrak{M}_{\mathrm{s}}$ and $\mathfrak{M}_{\mathrm{r}}$ of $\mathfrak{M}$ such that $\mathfrak{M}=\mathfrak{M}_{\mathrm{s}} \oplus \mathfrak{M}_{\mathrm{r}}$, and

$$
\begin{aligned}
& \mathfrak{M}_{\mathrm{s}}=\left\{x \in \mathfrak{M}: 0 \in{\overline{\left\{T_{t}(x): t \geq 0\right\}}}^{\mathrm{w}^{*}}\right\}, \\
& \mathfrak{M}_{\mathrm{r}}=\varlimsup_{\operatorname{lin}\left\{x \in \mathfrak{M}: \exists \alpha \in \mathbb{R} \text { such that } T_{t}(x)=\mathrm{e}^{\mathrm{i} \alpha t} x \forall t \geq 0\right\}}{ }^{\mathrm{w}^{*}} .
\end{aligned}
$$

Proof. First use Proposition 1.22 and Lemma 1.20 to conclude that the pointwise ultraweak closure of $\left\{T_{t}: t \geq 0\right\}$ in $\mathrm{L}(\mathfrak{M})$ consists of ultraweakultraweak continuous (i. e. normal) operators. Then apply Theorem 2.8. $\square$

We also have a result similar to Corollary 2.9.

Corollary 2.12 Assume that $\left\{T_{t}\right\}_{t \geq 0}$ is an ultraweakly continuous semigroup on $\mathfrak{M}$ such that each $T_{t}$ is strongly positive, and that $\omega \circ T_{t} \leq \omega$ for a faithful normal state $\omega$ on $\mathfrak{M}$. Then the conclusion of Corollary 2.11 holds, $\mathfrak{M}_{\mathrm{r}}$ is a von Neumann subalgebra of $\mathfrak{M}$ and there exists a group of ${ }^{*}$-automorphisms $\left\{\alpha_{t}\right\}_{t \in \mathbb{R}} \subseteq$ Aut $\mathfrak{M}_{\mathrm{r}}$ such that $T_{t} \uparrow \mathfrak{M}_{\mathrm{r}}=\alpha_{t}$ for all $t \geq 0$. 
Proof. Let $M_{0}=\left\{x \in \mathfrak{M}: \exists \alpha \in \mathbb{R}\right.$ such that $\left.T_{t}(x)=\mathrm{e}^{\mathrm{i} \alpha t} x \forall t \geq 0\right\}$, i. e. we have ${\overline{\operatorname{lin}} M_{0}}{ }^{*}=\mathfrak{M}_{\mathrm{r}}$. We define the sesquilinear map $D: \mathfrak{M} \times$ $\mathfrak{M} \longrightarrow \mathfrak{M}$ by $D(x, y)=T_{t}\left(x^{*} y\right)-T_{t}(x)^{*} T_{t}(y)$. By Kadison's inequality, $T_{t}\left(x^{*} x\right) \geq T_{t}\left(x^{*}\right) T_{t}(x)$, it follows that $D$ is positive-definite. Moreover, for any $\varphi \in \mathfrak{M}_{*}^{+}$the sesquilinear form $\varphi \circ D$ is positive-definite, so by the Cauchy-Schwarz inequality, $D(x, x)=0$ implies $D(x, y)=0$ for all $y \in \mathfrak{M}$. Now let $x \in M_{0}$, then $T_{t}\left(x^{*} x\right) \geq T_{t}(x)^{*} T_{t}(x)=\mathrm{e}^{-\mathrm{i} \alpha t} \mathrm{e}^{+\mathrm{i} \alpha t} x^{*} x=x^{*} x$. Thus $0 \leq \omega\left(T_{t}\left(x^{*} x\right)-x^{*} x\right) \leq \omega\left(x^{*} x-x^{*} x\right)=0$, and by faithfulness $T_{t}\left(x^{*} x\right)=x^{*} x$, hence $D(x, x)=0$ for all $x \in M_{0}$. By what we have shown above we get $D(x, y)=0$ for all $x, y \in M_{0}$, that is $T_{t}\left(x^{*} y\right)=T_{t}(x)^{*} T_{t}(y)=$ $\mathrm{e}^{\mathrm{i}\left(\alpha_{2}-\alpha_{1}\right) t} x^{*} y$, and thus $x y \in M_{0}$ whenever $x, y \in M_{0}$; moreover, $M_{0}$ is clearly ${ }^{*}$-invariant, thus lin $M_{0}$ is a ${ }^{*}$-subalgebra of $\mathfrak{M}$. Then clearly $\mathfrak{M}_{\mathrm{r}}$ is a von Neumann subalgebra.

Under the assumptions Lemma 2.4 applies and it is easy to see that $\mathfrak{M}_{\mathrm{r}} \subseteq \mathfrak{M}_{1}$. Since $\mathfrak{M}_{\mathrm{r}}$ is $T_{t}$-invariant the conclusion follows.

Remark 2.13 Assume that the conditions of the previous corollary are satisfied. From the proof of Theorem 2.8 we infer that there exists a projection $E$ in $\mathrm{L}(\mathfrak{M})$ onto $\mathfrak{M}_{\mathrm{r}}$ (the unit of the Sushkevich kernel of $\mathrm{S}$, where $\mathrm{S}$ is the closure of $\mathrm{S}_{0}=\left\{T_{t}: t \geq 0\right\}$ ). Then $E$ is strongly positive, normal, and satisfies $\left[T_{t}, E\right]=0$ for any $t \geq 0$ as well as $\omega \circ E \leq \omega$, hence $\omega \circ E=\omega$ by Proposition 1.31. Since $E(\mathbb{1})=\mathbb{1}$ we get $\|E\| \geq 1$, and since $E \in S \subseteq$ ball $\mathrm{L}(\mathfrak{M})$ we get $\|E\| \leq 1$, thus $\|E\|=1$, so $E$ is a normal conditional expectation by Tomiyama's theorem.

Next we give some sufficient conditions under which we obtain a stronger characterization of the subspace $\mathfrak{M}_{\mathrm{s}}$ as in Proposition 2.10.

Proposition 2.14 Assume that the hypotheses of Corollary 2.11 are satisfied. Moreover, assume that $\mathfrak{M}_{\mathrm{s}}$ is a von Neumann subalgebra of $\mathfrak{M}$. Then

$$
\mathfrak{M}_{\mathrm{s}}=\left\{x \in \mathfrak{M}: \lim _{t \rightarrow \infty} T_{t}(x)=0 \text { ultraweakly }\right\} .
$$

Proof. We will use the $\mathrm{s}^{*}\left(\mathfrak{M}, \mathfrak{M}_{*}\right)$-topology which is induced by the family of seminorms $\left\{p_{\omega}(x)=\left[\omega\left(x x^{*}\right)\right]^{1 / 2}, p_{\omega}^{*}(x)=\left[\omega\left(x^{*} x\right)\right]^{1 / 2}: \omega \in \mathfrak{M}_{*}^{+}\right\}$, it agrees with $\tau\left(\mathfrak{M}, \mathfrak{M}_{*}\right)$ on ball $\mathfrak{M}$. Let $x \in \mathfrak{M}$ such that 0 is an ultraweak limit point of $\left\{T_{t}(x): t \geq 0\right\}$, and assume first that $x \geq 0$. There exists a net $\left\{t_{i}\right\}_{i \in I} \subseteq \mathbb{R}^{+}$such that $y_{i}=T_{t_{i}}(x) \rightarrow 0$ relative to the ultraweak topology, and $y_{i} \geq 0$. Therefore we have by the Cauchy-Schwarz inequality

$$
p_{\omega}\left(y_{i}\right)^{2}=p_{\omega}^{*}\left(y_{i}\right)^{2}=\omega\left(y_{i}^{*} y_{i}\right)=\omega\left(y_{i}^{2}\right) \leq\left\|y_{i}\right\| \omega\left(y_{i}\right) \leq\|x\| \omega\left(y_{i}\right) \rightarrow 0 .
$$


We conclude that $y_{i} \rightarrow 0$ relative to $\tau\left(\mathfrak{M}, \mathfrak{M}_{*}\right)$. Now choose $\varphi \in \mathfrak{M}_{*}$ and let $K=\overline{\operatorname{co}}\left\{\left(T_{t}\right)_{*}(\varphi): t \geq 0\right\}$. Then by Lemma 1.20 the set $K \subseteq \mathfrak{M}_{*}$ is $\sigma\left(\mathfrak{M}_{*}, \mathfrak{M}\right)$ compact and convex. We will denote the seminorms of the $\tau\left(\mathfrak{M}, \mathfrak{M}_{*}\right)$ topology by $p_{K}(x)=\sup \{|\langle x, \psi\rangle|: \psi \in K\}$, where $K \subseteq \mathfrak{M}_{*}$ is weakly compact and convex. Now for $\epsilon>0$ there is $i_{0} \in I$ such that $p_{K}\left(T_{t_{i}}(x)\right)<\epsilon$ whenever $i \geq i_{0}$. If $t \geq t_{i_{0}}$ then

$$
\left|\left\langle T_{t}(x), \varphi\right\rangle\right|=\left|\left\langle T_{t_{i_{0}}}(x),\left(T_{t-t_{i_{0}}}\right)_{*}(\varphi)\right\rangle\right| \leq \sup _{\psi \in K}\left|\left\langle T_{t_{i_{0}}}(x), \psi\right\rangle\right|<\epsilon,
$$

and $T_{t}(x) \rightarrow 0$ as desired when $t \rightarrow \infty$. Next we consider an arbitrary $x \in \mathfrak{M}_{\mathrm{s}}$. Then $x$ can be written as a linear combination $x=\sum_{k=1}^{4} \lambda_{k} x_{k}$ of four positive elements. Since, by assumption, $\mathfrak{M}_{\mathrm{s}}$ is a von Neumann algebra, we have $x_{1}, \ldots, x_{4} \in \mathfrak{M}_{\mathrm{s}}$ and the assertion follows from what we have already proved.

Proposition 2.15 Assume that the hypotheses of Corollary 2.12 are satisfied. Additionally, assume that $\mathfrak{M}_{\mathrm{r}}=\mathfrak{M}_{1}$, where $\mathfrak{M}_{1}$ is defined by (2.9). Then $\mathfrak{M}_{\mathrm{s}}$ is given by (2.27).

Proof. Let $x \in \mathfrak{M}_{\mathrm{s}}$ and assume without loss of generality that $\|x\| \leq 1$. By Alaoglu's theorem the net $\left\{T_{t}(x)\right\}_{t \in \mathbb{R}^{+}} \subseteq$ ball $\mathfrak{M}$ has a limit point $x_{0}$ for $t \rightarrow \infty$. Then using Lemma 2.6 we find that $x_{0} \in \mathfrak{M}_{\mathrm{r}}=\mathfrak{M}_{1}$. But since $x \in \mathfrak{M}_{\mathrm{s}}$, it follows that also $x_{0} \in \mathfrak{M}_{\mathrm{s}}$, i. e. $x_{0} \in \mathfrak{M}_{\mathrm{s}} \cap \mathfrak{M}_{\mathrm{r}}=\{0\}$, hence $x_{0}=0$. This proves that any limit point of the net $\left\{T_{t}(x)\right\}_{t \in \mathbb{R}^{+}}$is equal to 0 , therefore we conclude that $\lim _{t \rightarrow \infty} T_{t}(x)=0$ relative to the ultraweak topology for all $x \in \mathfrak{M}_{\mathrm{s}}$.

Remark 2.16 Suppose that the assumptions of Theorem 2.3 are satisfied. If $\mathfrak{M}_{1}=\mathfrak{M}_{\mathrm{r}}$, then the isometric-sweeping decomposition and the Jacobsde Leeuw-Glicksberg splitting agree. This follows from Remark 2.13 and the fact that the condition $\omega \circ E=\omega$ uniquely determines a conditional expectation, cf. [4], Corollary II.6.10.8.

Remark 2.17 If in the situation of Corollary 2.11 the isometric-sweeping decomposition exists as well it is not necessary true that both splittings agree, even if the the stable subspace $\mathfrak{M}_{\mathrm{s}}$ of the Jacobs-de Leeuw-Glicksberg splitting is given by (2.27). In fact, one can construct examples in which the semigroup $\left\{T_{t}\right\}_{t \geq 0}$ is a group of automorphisms such that $\mathfrak{M}_{\mathrm{r}}=\mathbb{C} \mathbb{1}$ whereas, of course, $\mathfrak{M}_{1}=\mathfrak{M}$ (recall that in the isometricsweeping decomposition the subalgebra $\mathfrak{M}_{1}$ was defined as the largest von Neumann subalgebra on which each $T_{t}$ is given by an automorphism). 
For this reason (cf. the discussion in Section 3.2) it is the isometricsweeping decomposition that is relevant in applications to decoherence. However, Proposition 2.15 and Remark 2.16 show that the Jacobs-de Leeuw-Glicksberg splitting may be of some value in situations in which the conditions of Theorem 2.3 are hard to verify. We will give an illustrative example in the following subsection.

\subsubsection{An Example}

In this subsection we give an example which shows the utility of the Jacobs-de Leeuw-Glicksberg splitting, given in Corollary 2.11 and Proposition 2.15 , in the study of decoherence.

Let $\mathfrak{M}$ be a von Neumann algebra acting on the Hilbert space $\mathscr{H}$ and $\left\{T_{t}\right\}_{t \geq 0}$ semigroup of normal completely positive contractive and unital operators. Suppose that $t \mapsto T_{t}(x)$ is continuous in the uniform topology. Then by [57] the generator $Z$ of $\left\{T_{t}\right\}_{t \geq 0}$, which is a bounded operator on $\mathfrak{M}$, is given by

$$
Z x=G^{*} x+x G+\Phi(x),
$$

where $G \in \mathrm{L}(\mathscr{H})$ and $\Phi: \mathfrak{M} \longrightarrow \mathrm{L}(\mathscr{H})$ is a completely positive map. Since we have $T_{t}(\mathbb{1})=\mathbb{1}$ for all $t \geq 0$ it follows that $Z \mathbb{1}=0$ which forces $G^{*}=-G-\Phi(\mathbb{1})$. Introduce the operator $H=\mathrm{i} G+\frac{1}{2} \mathrm{i} \Phi(\mathbb{1})$, then $H$ is a bounded self-adjoint operator on $\mathscr{H}$ and $Z$ may be written as

$$
Z x=\mathrm{i}[H, x]-\frac{1}{2}\{\Phi(\mathbb{1}), x\}+\Phi(x),
$$

here $\{\cdot, \cdot\}$ denotes the anticommutator. By Kraus' theorem there exists a measure space $(\Omega, \mathscr{F}, \mu)$ and a strongly measurable map $\Omega \ni \omega \mapsto A_{\omega} \in$ $\mathrm{L}(\mathscr{H})$ such that

$$
\Phi(x)=\int_{\Omega} A_{\omega}^{*} x A_{\omega} \mathrm{d} \mu(\omega),
$$

the integral is to be understood as a weak* integral. In general we can take $\mu$ to be discrete, but let us write $\Phi$ as a $\mu$-integral as above. Then by a simple calculation [66] one obtains

$$
\begin{aligned}
Z\left(x^{*} x\right)-Z\left(x^{*}\right) x-x^{*} Z(x) & =x^{*} \Phi(\mathbb{1}) x+\Phi\left(x^{*} x\right)-\Phi\left(x^{*}\right) x-x^{*} \Phi(x) \\
& =\int_{\Omega}\left[A_{\omega}, x\right]^{*}\left[A_{\omega}, x\right] \mathrm{d} \mu(\omega) .
\end{aligned}
$$

We are now in a position to prove the following proposition. 
Proposition 2.18 Let $\left\{T_{t}\right\}_{t \geq 0}$ be as above. Assume that there is a faithful normal state $\omega$ such that $\omega \circ T_{t}=\omega$ for all $t \geq 0$ and that $H$ in (2.29) has pure point spectrum. Then the conclusion of Theorem 2.3 holds and the von Neumann subalgebra $\mathfrak{M}_{1}$ defined by $(2.9)$ is given by

$$
\mathfrak{M}_{1}=\left\{A_{\omega}, A_{\omega}^{*}: \omega \in \Omega\right\}^{\prime} \cap \mathfrak{M},
$$

where the commutant is to be interpreted in a $\mu$-almost everywhere sense.

Proof. First note that the assumptions of Lemma 2.4 and Corollary 2.12 are satisfied, i. e. $\mathfrak{M}_{\mathrm{r}}$ is a von Neumann subalgebra on which $\left\{T_{t}\right\}_{t \geq 0}$ is given by a group of automorphisms, moreover, $\mathfrak{M}_{1}$ is the image of a normal conditional expectation commuting with each $T_{t}$, cf. Remark 2.13. We prove (2.31). The generator $Z$, when restricted to $\mathfrak{M}_{1}$, is a ${ }^{*}$-derivation, thus if $x \in \mathfrak{M}_{1}$ then

$$
0=Z\left(x^{*} x\right)-x^{*} Z(x)-Z\left(x^{*}\right) x=\int_{\Omega}\left[A_{\omega}, x\right]^{*}\left[A_{\omega}, x\right] \mathrm{d} \mu(\omega)
$$

by (2.30), i.e. $\left[A_{\omega}, x\right]=0 \mu$-almost everywhere, similarly $\left[A_{\omega}^{*}, x\right]=0 \mu$ almost everywhere. This proves that $\mathfrak{M}_{1} \subseteq\left\{A_{\omega}, A_{\omega}^{*}: \omega \in \Omega\right\}^{\prime} \cap \mathfrak{M}$. Conversely, if $x \in\left\{A_{\omega}, A_{\omega}^{*}: \omega \in \Omega\right\}^{\prime} \cap \mathfrak{M}$ then $Z x=\mathrm{i}[H, x]$, thus $T_{t}(x)=$ $\mathrm{e}^{\mathrm{i} t H} x \mathrm{e}^{-\mathrm{i} t H}$ and consequently $x \in \mathfrak{M}_{1}$.

Now let $x \in \mathfrak{M}_{\mathrm{s}} \cap \mathfrak{M}_{1}, x \neq 0$. Then there exist eigenvectors $\xi, \eta \in \mathscr{H}$ of $H$ such that $\langle\xi, x \eta\rangle \neq 0$, thus

$$
\left\langle\xi, T_{t}(x) \eta\right\rangle=\left\langle\mathrm{e}^{-\mathrm{i} t H} \xi, x \mathrm{e}^{-\mathrm{i} t H} \eta\right\rangle=\mathrm{e}^{\mathrm{i} t\left(E_{\xi}-E_{\eta}\right)}\langle\xi, x \eta\rangle,
$$

is bounded away from 0 , so 0 is not a weak* limit point of $\left\{T_{t}(x): t \geq 0\right\}$. Since $x \in \mathfrak{M}_{\mathrm{s}}$ this is a contradiction in view of (2.25), hence $\mathfrak{M}_{\mathrm{s}} \cap \mathfrak{M}_{1}=$ $\{0\}$. Now let $x \in \mathfrak{M}_{1}$ and write $x=x_{\mathrm{s}}+x_{\mathrm{r}} \in \mathfrak{M}_{\mathrm{s}} \oplus \mathfrak{M}_{\mathrm{r}}$. Since $\mathfrak{M}_{\mathrm{r}} \subseteq \mathfrak{M}_{1}$ we have $x_{\mathrm{r}} \in \mathfrak{M}_{1}$, and $x_{\mathrm{s}}=x-x_{\mathrm{r}} \in \mathfrak{M}_{\mathrm{s}} \cap \mathfrak{M}_{1}=\{0\}$, thus $x \in \mathfrak{M}_{\mathrm{r}}$. This proves $\mathfrak{M}_{1}=\mathfrak{M}_{\mathrm{r}}$ and we conclude by Proposition 2.15 that $\mathfrak{M}_{\mathrm{s}}$ has the property (2.27). So all conclusions of Theorem 2.3 hold.

We remark that the last proposition can be generalized to certain cases when the semigroup $\left\{T_{t}\right\}_{t \geq 0}$ is not uniformly continuous but has an unbounded generator of the form (2.29).

In the application of the previous proposition question arises under which conditions the semigroup $\left\{T_{t}\right\}_{t \geq 0}$ has a faithful normal invariant state, and a criterion in terms of the generator $Z$ would be desirable. One possibility to obtain a criterion is to proceed as follows. The predual of $Z$ can be written as

$$
Z_{*}(x)=-\mathrm{i}[H, \rho]-\frac{1}{2}\left\{\Phi^{*}(\mathbb{1}), \rho\right\}+\Phi^{*}(\rho),
$$


where $\rho$ is a density matrix on $\mathscr{H}$ and

$$
\Phi^{*}(\rho)=\int_{\Omega} A_{\omega} \rho A_{\omega}^{*} \mathrm{~d} \mu(\omega) .
$$

Clearly $Z_{*}$ extends to $\mathrm{T}(\mathscr{H})$ and preserves $\mathfrak{M}_{*}$. Let $\operatorname{spec}_{\mathrm{P}}\left(Z_{*}\right)$ denote the point spectrum of $Z_{*}$. Then according to U. Groh's contribution in [18], there exists a faithful normal invariant state $\omega$ for $\left\{T_{t}\right\}_{t \geq 0}$ provided $\operatorname{spec}_{\mathrm{p}}\left(Z_{*}\right) \cap i \mathbb{R} \neq \emptyset$.

If the von Neumann algebra $\mathfrak{M}$ is finite-dimensional, i. e. acts on a finitedimensional Hilbert space, we get a particularly satisfying result.

Corollary 2.19 Let $\mathfrak{M}$ be a finite-dimensional von Neumann algebra and $\left\{T_{t}\right\}_{t \geq 0}$ be a semigroup of completely positive unital maps such that the map $\mathbb{R}^{+} \ni t \mapsto T_{t}(x)$ is continuous for any $x \in \mathfrak{M}$. Then the conclusion of Theorem 2.3 holds and the von Neumann subalgebra $\mathfrak{M}_{1}$ defined by (2.9) is given by (2.31).

Proof. If $\mathfrak{M}$ is finite-dimensional the ultraweak and norm topologies on $\mathfrak{M}$ coalesce, and the predual generator $Z_{*}$ of $\left\{T_{t, *}\right\}_{t>0}$ is given by (2.32). Assume that $\mathfrak{M}$ acts on the Hilbert space $\mathbb{C}^{n}$. Then $\omega(x)=n^{-1} \operatorname{tr} x$ defines a faithful (normal) state on $\mathfrak{M}$, and since $Z_{*}\left(n^{-1} \mathbb{1}\right)=0$ from $(2.32)$ it is invariant under $\left\{T_{t}\right\}_{t \geq 0}$. Hence Proposition 2.18 applies.

Of course, since the state $\omega(x)=n^{-1}$ tr $x$ is tracial we can obtain the same conclusion also from Theorem 2.3 since then $\sigma_{t}^{\omega}=\mathrm{id}$.

\subsection{Detailed Balance}

\subsubsection{Definition and Properties}

In classical statistical mechanics the notion of detailed balance is wellknown. Roughly we say that a state satisfies detailed balance if the probability in this state to jump from a phase space point $x_{1}$ to a point $x_{2}$ equals the probability for the inverse jump from $x_{2}$ to $x_{1}$ at all times. This situation is also called microscopic reversibility. In the literature various noncommutative generalizations of the detailed balance condition have been introduced $[34,55,36,86,96,97,133,99,68]$, in most cases for Markovian evolutions. We will use the detailed balance condition introduced in [99] for a single dynamical map.

Definition 2.20 Suppose that $\mathfrak{A}$ is a unital $C^{*}$-algebra. Let $T \in \mathrm{L}(\mathfrak{A})$ be a positive unital map and $\omega$ a state on $\mathfrak{A}$. We say that $T$ satisfies detailed 
balance II with respect to $\omega$ if there exists a positive unital map $T^{\beta} \in \mathrm{L}(\mathfrak{A})$ such that

$$
\omega(x T(y))=\omega\left(T^{\beta}(x) y\right) \quad \text { for all } x, y \in \mathfrak{A} .
$$

Most of the time we will use this definition in the situation when $\mathfrak{A}$ is a von Neumann algebra, $\omega$ a normal state and $T, T^{\beta}$ normal maps. We next introduce detailed blance II for semigroups.

Definition 2.21 Let $\mathfrak{A}$ be a unital $C^{*}$-algebra, $\left\{T_{t}\right\}_{t \geq 0} \in \mathrm{L}(\mathfrak{A})$ be a semigroup of positive unital maps and $\omega$ a state on $\mathfrak{A}$. Then we say that $\left\{T_{t}\right\}_{t>0}$ satisfies detailed balance II with respect to $\omega$ if there exist positive unital maps $T_{t}^{\beta} \in \mathrm{L}(\mathfrak{A})$ such that

$$
\omega\left(x T_{t}(y)\right)=\omega\left(T_{t}^{\beta}(x) y\right) \quad \text { for all } x, y \in \mathfrak{A}, t \geq 0 .
$$

We next show that $\left\{T_{t}^{\beta}\right\}_{t \geq 0}$ must be a semigroup as well provided $\omega$ is faithful.

Lemma 2.22 Let $\mathfrak{A}$ be a unital $C^{*}$-algebra and $\left\{T_{t}\right\}_{t \geq 0} \subseteq \mathrm{L}(\mathfrak{A})$ a semigroup of positive unital maps which satisfies detailed balance II with respect to a faithful state $\omega$ on $\mathfrak{A}$. Then $\left\{T_{t}^{\beta}\right\}_{t \geq 0}$ is a semigroup as well.

Proof. Let $\left(\pi_{\omega}, \mathscr{H}_{\omega}, \xi_{\omega}\right)$ be the GNS triplet, then $\pi_{\omega}$ is a faithful representation and if $\pi_{\omega}(x) \xi_{\omega}=0$ it follows that $0=\left\|\pi_{\omega}(x) \xi_{\omega}\right\|^{2}=\omega\left(x^{*} x\right)$ and we obtain $x=0$. Now for $s, t \geq 0$,

$$
\begin{aligned}
\omega\left(T_{s+t}^{\beta}(x) y\right) & =\omega\left(x T_{s+t}(y)\right)=\omega\left(x T_{s}\left(T_{t}(y)\right)\right) \\
& =\omega\left(T_{s}^{\beta}\left(T_{t}^{\beta}(x)\right) y\right)
\end{aligned}
$$

for all $x, y \in \mathfrak{A}$, hence

$$
\left\langle\pi_{\omega}\left(T_{s+t}^{\beta}(x)\right)^{*} \xi_{\omega}, \pi_{\omega}(y)\right\rangle=\left\langle\pi_{\omega}\left(T_{s}^{\beta}\left(T_{t}^{\beta}(x)\right)\right)^{*} \xi_{\omega}, \pi_{\omega}(y) \xi_{\omega}\right\rangle
$$

for all $x, y \in \mathfrak{A}$, consequently $\pi_{\omega}\left(T_{s+t}^{\beta}(x)\right)^{*} \xi_{\omega}=\pi_{\omega}\left(T_{s}^{\beta}\left(T_{t}^{\beta}(x)\right)\right)^{*} \xi_{\omega}$. By what was shown above, $\pi_{\omega}\left(T_{s+t}^{\beta}(x)\right)=\pi_{\omega}\left(T_{s}^{\beta}\left(T_{t}^{\beta}(x)\right)\right)$, and since $\pi_{\omega}$ is faithful we arrive at $T_{s+t}^{\beta}(x)=T_{s}^{\beta}\left(T_{t}^{\beta}(x)\right)$.

The next result, proved in [99], states that if $T$ satisfies detailed balance it extends to a contraction on the GNS Hilbert space with respect to $\omega$.

Proposition 2.23 Suppose that $\mathfrak{A}$ is $C^{*}$-algebra, let $T \in \mathrm{L}(\mathfrak{A})$ be a positive unital map and $\omega$ a state on $\mathfrak{A}$ such that $T$ satisfies detailed balance II 
with respect to $\omega$. Let $\left(\pi_{\omega}, \mathscr{H}_{\omega}, \xi_{\omega}\right)$ be the GNS triplet, then there exists a contraction $\hat{T}$ on $\mathscr{H}_{\omega}$ such that

$$
\hat{T}\left(\pi_{\omega}(x) \xi_{\omega}\right)=\pi_{\omega}(T(x)) \xi_{\omega}
$$

for all $x \in \mathfrak{A}$.

Proof. Define $\hat{T}$ on $\pi_{\omega}(\mathfrak{A}) \xi_{\omega}$ by (2.35). Now since $T^{\beta}$ is positive and unital, it is a contraction, hence

$$
\begin{aligned}
\left\|\hat{T}\left(\pi_{\omega}(x) \xi_{\omega}\right)\right\|^{2} & =\omega\left(T(x)^{*} T(x)\right) \\
& =\omega\left(T^{\beta}\left(T\left(x^{*}\right)\right) x\right) \\
& \leq \omega\left[T^{\beta}\left(T\left(x^{*}\right)\right) T^{\beta}(T(x))\right]^{1 / 2} \omega\left(x^{*} x\right)^{1 / 2} \\
& =\omega\left[\left(T^{\beta} \circ T \circ T^{\beta} \circ T\right)\left(x^{*}\right) x\right]^{1 / 2} \omega\left(x^{*} x\right)^{1 / 2} \\
& \leq \omega\left[\left(T^{\beta} \circ T\right)^{2}\left(x^{*}\right)\left(T^{\beta} \circ T\right)^{2}(x)\right]^{1 / 4} \omega\left(x^{*} x\right)^{3 / 4} \\
& \vdots \\
& \leq \omega\left[\left(T^{\beta} \circ T\right)^{n}\left(x^{*}\right)\left(T^{\beta} \circ T\right)^{n}(x)\right]^{1 / 2^{n}} \omega\left(x^{*} x\right)^{1-1 / 2^{n}}
\end{aligned}
$$

for any $n \in \mathbb{N}$. Now

$$
\begin{aligned}
\left|\omega\left[\left(T^{\beta} \circ T\right)^{n}\left(x^{*}\right)\left(T^{\beta} \circ T\right)^{n}(x)\right]\right|^{1 / 2^{n}} & \leq\left\|\left(T^{\beta} \circ T\right)^{n}(x)\right\|^{1 / 2^{n-1}} \\
& \leq\|x\|^{1 / 2^{n-1}} \rightarrow 1
\end{aligned}
$$

as $n \rightarrow \infty$. Moreover, as $n \rightarrow \infty$ we get $\omega\left(x^{*} x\right)^{1-1 / 2^{n}} \rightarrow \omega\left(x^{*} x\right)$, consequently $\left\|\hat{T}\left(\pi_{\omega}(x) \xi_{\omega}\right)\right\| \leq\left\|\pi_{\omega}(x) \xi_{\omega}\right\|$ for any $x \in \mathfrak{A}$ and $\hat{T}$ extends to a contraction on $\mathscr{H}_{\omega}$.

Next we show that if $T$ is a normal map on a von Neumann algebra which satisfies detailed balance with respect to a faithful normal state then $T^{\beta}$ is $n$-positive if $T$ is $n$-positive.

Proposition 2.24 Let $\mathfrak{M}$ be a von Neumann algebra and $\omega$ a faithful normal state on $\mathfrak{M}$. Suppose that a normal positive map $T \in \mathrm{L}(\mathfrak{M})$ satisfies detailed balance II with respect to $\omega$. Then if $T$ is n-positive, $T^{\beta}$ is n-positive as well.

Proof. Without loss of generality we can assume that $\omega(x)=\left\langle\xi_{0}, x \xi_{0}\right\rangle$ for $x \in \mathfrak{M}$ where $\xi_{0}$ is a cyclic and separating vector for $\mathfrak{M}$. Let $\left\{\sigma_{t}^{\omega}\right\}_{t \in \mathbb{R}}$ be the modular group associated with $\xi_{0}$ and let $x_{1}, \ldots, x_{n}, y_{1}, \ldots, y_{n} \in \mathfrak{M}_{\mathrm{e}}$ 
and $n \in \mathbb{N}$. By Theorem 2.29 $T^{\beta}$ commutes with the modular group, hence

$$
\begin{aligned}
\left\langle y_{i} \xi_{0}, T^{\beta}\left(x_{i}^{*} x_{j}\right) y_{j} \xi_{0}\right\rangle & =\left\langle\xi_{0}, y_{i}^{*} T^{\beta}\left(x_{i}^{*} x_{j}\right) y_{j} \xi_{0}\right\rangle \\
& =\left\langle\xi_{0}, \sigma_{+\mathrm{i}}\left(y_{j}\right) y_{i}^{*} T^{\beta}\left(x_{i}^{*} x_{j}\right) \xi_{0}\right\rangle \\
& =\left\langle\xi_{0}, T\left(\sigma_{+\mathrm{i}}\left(y_{j}\right) y_{i}^{*}\right) x_{i}^{*} x_{j} \xi_{0}\right\rangle \\
& =\left\langle\xi_{0}, \sigma_{-\mathrm{i} / 2}\left[T\left(\sigma_{\mathrm{i}}\left(y_{j}\right) y_{i}^{*}\right) x_{i}^{*} x_{j}\right] \xi_{0}\right\rangle \\
& =\left\langle\xi_{0}, T\left[\sigma_{-\mathrm{i} / 2}\left(\sigma_{\mathrm{i}}\left(y_{j}\right) y_{i}^{*}\right)\right] \sigma_{-\mathrm{i} / 2}\left(x_{i}^{*}\right) \sigma_{-\mathrm{i} / 2}\left(x_{j}\right) \xi_{0}\right\rangle \\
& =\left\langle\xi_{0}, \sigma_{\mathrm{i} / 2}\left(x_{j}\right) T\left[\sigma_{\mathrm{i} / 2}\left(y_{j}\right) \sigma_{\mathrm{i} / 2}\left(y_{i}\right)^{*}\right] \sigma_{\mathrm{i} / 2}\left(x_{i}\right)^{*} \xi_{0}\right\rangle,
\end{aligned}
$$

hence

$$
\sum_{i, j=1}^{n}\left\langle\xi_{0}, y_{i}^{*} T^{\beta}\left(x_{i}^{*} x_{j}\right) y_{j} \xi_{0}\right\rangle \geq 0
$$

by $n$-positivity of $T$. Since $\mathfrak{M}_{\mathrm{e}} \subseteq \mathfrak{M}$ is ultraweakly dense and $T^{\beta}$ is normal it follows that the above inequality holds for all $x_{1}, \ldots, x_{n}, y_{1}, \ldots, y_{n}$ in $\mathfrak{M}$. Now choose $a \in \mathfrak{M}$ and replace $y_{i}$ by $y_{i} a$ in the above equation, where $i=1, \ldots, n$, then we find

$$
\sum_{i, j=1}^{n}\left\langle a \xi_{0}, y_{i}^{*} T^{\beta}\left(x_{i}^{*} x_{j}\right) y_{j}\left(a \xi_{0}\right)\right\rangle \geq 0
$$

hence $\sum_{i, j=1}^{n} y_{i}^{*} T^{\beta}\left(x_{i}^{*} x_{j}\right) y_{j} \geq 0$ because $\mathfrak{M} \xi_{0} \subseteq \mathscr{H}$ is dense. Thus we have proved that $T^{\beta}$ is $n$-positive whenever $T$ is $n$-positive.

In the algebraic framework there is another version of detailed balance, different from the one given in Definition 2.20. Following [99] we shall call it "detailed balance I". It is of less relevance to the present work and is mentioned merely for completeness. Originally it was introduced in [97], using ideas from [34].

Definition 2.25 Let $\mathfrak{A}$ be a $C^{*}$-algebra and $\omega$ a state on $\mathfrak{A}$. An antilinear Jordan automorphism ${ }^{2} \sigma$ of $\mathfrak{A}$ of order 2 (i. e. $\left.\sigma^{2}=\mathrm{id}\right)$ is called a reversing operation if it satisfies

$$
\begin{aligned}
\omega(\sigma(x) \sigma(y)) & =\omega(\sigma(x y)), \\
\omega\left(\sigma\left(y^{*}\right) \sigma(x)\right) & =\omega\left(x^{*} y\right),
\end{aligned}
$$

${ }^{2}$ That is, $\sigma$ is antilinear and satisfies $\sigma\left(x^{*}\right)=\sigma(x)^{*}$ as well as $\sigma\{x, y\}=\{\sigma(x), \sigma(y)\}$ for all $x, y \in \mathfrak{A}$, where $\{x, y\}=x y+y x$. 
for all $x, y \in \mathfrak{A}$. A positive unital map $T \in \mathrm{L}(\mathfrak{A})$ is said to satisfy detailed balance I with respect to $\omega$ and $\sigma$ provided

$$
\omega\left(x^{*} T(y)\right)=\omega\left(\sigma\left(y^{*}\right) T(\sigma(x))\right) \text { for all } x, y \in \mathfrak{A} .
$$

For dynamical semigroups $\left\{T_{t}\right\}_{t \geq 0}$ on $\mathfrak{A}$ we also introduce detailed balance $\mathrm{I}$ in the natural way by requiring it to hold for each $T_{t}, t \geq 0$, for fixed $\omega$ and $\sigma$. There is a simple relation between detailed balance I and detailed balance II, which was observed in [99].

Proposition 2.26 Suppose that $T$ satisfies detailed balance I with respect to the state $\omega$ and the Jordan automorphism $\sigma$. Then it satisfies detailed balance II with respect to $\omega$.

Proof. Let $x, y \in \mathfrak{A}$. From (2.36) and (2.38) we obtain

$$
\begin{aligned}
\omega\left(x^{*} T(y)\right) & =\omega\left[\sigma(y)^{*} T(\sigma(x))\right] \\
& =\omega\left[\sigma\left(T\left(\sigma(x)^{*}\right)\right) \sigma(\sigma(y))\right] \\
& =\omega\left[(\sigma \circ T \circ \sigma)\left(x^{*}\right) y\right] \\
& =\omega\left(T^{\beta}\left(x^{*}\right) y\right),
\end{aligned}
$$

where $T^{\beta}=\sigma \circ T \circ \sigma$. Since a Jordan automorphism is positive, $T^{\beta}$ is positive as well, hence $T$ satisfies detailed balance II.

We are now going to show that if a semigroup on a $\mathrm{C}^{*}$-algebra $\mathfrak{A}$ satisfies detailed balance II, it can, under suitable assumptions, be extended to a weak* continuous semigroup on the von Neumann closure of a representations of $\mathfrak{A}$ on a Hilbert space which satisfies detailed balance as well.

Proposition 2.27 Let $\mathfrak{A}$ be a $C^{*}$-algebra and let $\omega$ be a separating state on $\mathfrak{A}$. Let $\left\{\tau_{t}\right\}_{t \geq 0}$ be a $\omega$-continuous (see Definition B.6) semigroup of positive unital maps on $\mathfrak{A}$, and suppose that $\left\{\tau_{t}\right\}_{t \geq 0}$ satisfies detailed balance II with respect to $\omega$. Let $\mathfrak{M}=\pi_{\omega}(\mathfrak{A})^{\prime \prime}$. Then $\left\{\tau_{t}\right\}_{t \geq 0}$ is $\omega$-covariant and the ultraweakly continuous semigroup $\left\{T_{t}\right\}_{t \geq 0}$ of positive unital operators on $\mathfrak{M}$, defined by (B.9), satisfies detailed balance II with respect to the normal state $\hat{\omega}(x)=\left\langle\xi_{\omega}, x \xi_{\omega}\right\rangle, x \in \mathfrak{M}$.

Proof. By detailed balance II we have $\omega \circ \tau_{t}=\omega$ and $\omega \circ \tau_{t}^{\beta}=\omega$ for all $t \geq 0$, in particular $N_{\omega}=N_{\omega \circ \tau_{t}}=N_{\omega \circ \tau_{t}^{\beta}}$. Hence it follows from Theorem B.7 that there exists an ultraweakly continuous semigroup $\left\{T_{t}\right\}_{t \geq 0}$ of positive unital operators on $\mathfrak{M}$ such that $T_{t}\left(\pi_{\omega}(x)\right)=\pi_{\omega}\left(\tau_{t}(x)\right)$ for all $x \in \mathfrak{A}$ and 
$t \geq 0$. In the same way for $\left\{\tau_{t}^{\beta}\right\}_{t \geq 0}$ there exists a semigroup $\left\{T_{t}^{\beta}\right\}_{t \geq 0}$ with similar properties. Now by the properties of the GNS representation

$$
\begin{aligned}
\hat{\omega}\left[\pi_{\omega}(x) T_{t}\left(\pi_{\omega}(y)\right)\right] & =\hat{\omega}\left[\pi_{\omega}\left(x \tau_{t}(y)\right)\right] \\
& =\omega\left(x \tau_{t}(y)\right)=\omega\left(\tau_{t}^{\beta}(y) x\right) \\
& =\hat{\omega}\left[T_{t}^{\beta}\left(\pi_{\omega}(y)\right) \pi_{\omega}(x)\right]
\end{aligned}
$$

for all $x, y \in \mathfrak{A}$ and $t \geq 0$. Since $\pi_{\omega}(\mathfrak{A}) \subseteq \mathfrak{M}$ is ultraweakly dense and since $T_{t}$ and $T_{t}^{\beta}$ as well as $\hat{\omega}$ are normal the detailed balance condition for $\left\{T_{t}\right\}_{t \geq 0}$ follows.

\subsubsection{Equivalent Characterization of Detailed Balance}

In this section we will establish a number of equivalent characterizations of detailed balance II (Definition 2.20) for faithful normal states $\omega$ and normal maps $T$ on a von Neumann algebra. We start with a lemma concerning the commutation of unbounded operators in Hilbert spaces.

Lemma 2.28 Let $\mathscr{H}$ be a Hilbert space and let $H$ be a self-adjoint operator and $B$ a bounded operator on $\mathscr{H}$. The following assertions are equivalent:

1. $H$ and $B$ commute strongly, i. e. all spectral projections of $H$ commute with $B$.

2. There exists a core $C \subseteq \operatorname{dom} H$ for $H$ such that $B(C) \subseteq C$ and $B H \xi=H B \xi$ for all $\xi \in C$.

3. We have $B(\operatorname{dom} H) \subseteq \operatorname{dom} H$ and $B H \xi=H B \xi$ for all $\xi \in \operatorname{dom} H$.

For a proof see [53]. In the situation of the above lemma, if $f: \mathbb{R} \longrightarrow \mathbb{C}$ is a Borel function and if $H$ and $B$ commute strongly then by Theorem XI.12.1. of [32], $f(H)$ and $B$ commute strongly.

In the following theorem we give a number of equivalent characterizations of detailed balance II. Some implications of the theorem have already been observed and proved in the literature, e.g. $1 . \Leftrightarrow 2$. is wellknown, $6 . \Rightarrow 1$. has been proved in [86], and 4., 5. $\Rightarrow 2$. can be concluded from [53], however, we are not aware of a statement or proof of it in the literature as given below, therefore we provide it here.

Theorem 2.29 Let $\mathfrak{M}$ be a von Neumann algebra acting on $\mathscr{H}, \omega$ a faithful normal state on $\mathfrak{M}$ (without loss of generality a vector state $\xi_{0}$ ) 
and $T \in \mathrm{L}(\mathfrak{M})$ a normal positive and unital linear map. Suppose that there exists a contraction $\hat{T}$ on $\mathscr{H}$ such that $\hat{T}\left(x \xi_{0}\right)=T(x) \xi_{0}$ for all $x \in \mathfrak{M}$. Let $J, \Delta$ and $\left\{\sigma_{t}^{\omega}\right\}_{t \in \mathbb{R}}$ be the canonical objects associated to $\omega$ by Tomita-Takesaki theory. Then the following assertions are equivalent:

1. $T$ commutes with the modular group, $\left[T, \sigma_{t}^{\omega}\right]=0$ for all $t \in \mathbb{R}$.

2. $\hat{T}$ commutes strongly with $\Delta$ and $J$.

3. $\hat{T}$ commutes with $J$.

4. $\hat{T}^{*}\left(\mathfrak{M}^{+} \xi_{0}\right) \subseteq \overline{\mathfrak{M}^{+} \xi_{0}}$.

5. $\hat{T}\left(\mathfrak{M}^{\prime+} \xi_{0}\right) \subseteq \overline{\mathfrak{M}^{\prime+} \xi_{0}}$.

6. T satisfies detailed balance II with respect to $\omega$.

Then $T^{\beta}$ is normal and we have $\hat{T}^{*}\left(x \xi_{0}\right)=T^{\beta}(x) \xi_{0}$ for all $x \in \mathfrak{M}$.

Proof. 1. $\Rightarrow 2$. From the assumption we have $\sigma_{t}^{\omega}(T(x))=\Delta^{\mathrm{i} t} T(x) \Delta^{-\mathrm{i} t}=$ $T\left(\Delta^{\mathrm{i} t} x \Delta^{-\mathrm{i} t}\right)=T\left(\sigma_{t}^{\omega}(x)\right)$ for any $x \in \mathfrak{M}$ and $t \in \mathbb{R}$, hence

$$
\Delta^{\mathrm{i} t} \hat{T}\left(x \xi_{0}\right)=\Delta^{\mathrm{i} t} T(x) \xi_{0}=T\left(\Delta^{\mathrm{i} t} x \Delta^{-\mathrm{i} t}\right) \xi_{0}=\hat{T}\left(\Delta^{\mathrm{i} t} x \xi_{0}\right),
$$

thus it follows that $\Delta=\left(\Delta^{\mathrm{i} t}\right)^{-\mathrm{i} t}$ commutes strongly with $\hat{T}$. Next we note that by positivity of $T$ we have

$$
S \hat{T}\left(x \xi_{0}\right)=S T(x) \xi_{0}=T(x)^{*} \xi_{0}=T\left(x^{*}\right) \xi_{0}=\hat{T}\left(S x \xi_{0}\right)
$$

i. e. $S$ and $\hat{T}$ commute on $\mathfrak{M} \xi_{0}$. Now $S=J \Delta^{1 / 2}$, and for $\xi \in \mathfrak{M} \xi_{0}$ (in fact, $\mathfrak{M} \xi_{0} \subseteq \operatorname{dom} \Delta^{1 / 2}$ is a core for $\Delta^{1 / 2}$ ) we get

$$
\hat{T}\left(J \Delta^{1 / 2} \xi\right)=\hat{T}(S \xi)=S \hat{T}(\xi)=J \hat{T}\left(\Delta^{1 / 2} \xi\right),
$$

and since $\Delta^{1 / 2}$ is injective it follows that $[\hat{T}, J]=0$.

2 . $\Rightarrow 1$. If $\Delta$ commutes strongly with $\hat{T}$ then

$$
\Delta^{\mathrm{i} t} T(x) \Delta^{-\mathrm{i} t} \xi_{0}=\Delta^{\mathrm{i} t} \hat{T}\left(x \xi_{0}\right)=\hat{T}\left(\Delta^{\mathrm{i} t} x \xi_{0}\right)=T\left(\Delta^{\mathrm{i} t} x \Delta^{-\mathrm{i} t}\right) \xi_{0},
$$

and since $\xi_{0}$ is separating it follows that $\sigma_{t}^{\omega}(T(x))=\Delta^{\mathrm{i} t} T(x) \Delta^{-\mathrm{i} t}=$ $T\left(\Delta^{\mathrm{i} t} x \Delta^{-\mathrm{i} t}\right)=T\left(\sigma_{t}^{\omega}(x)\right)$ for all $x \in \mathfrak{M}$ and $t \in \mathbb{R}$.

2. $\Rightarrow 3$. is trivial.

3. $\Rightarrow 4$. For the proof of this implication we first note the following facts which are proved in $[6]$ :

$$
\overline{\mathfrak{M}^{+} \xi_{0}}=\left\{\xi \in \mathscr{H}:\langle\xi, \eta\rangle \geq 0 \text { for all } \eta \in \overline{\mathfrak{M}^{\prime+} \xi_{0}}\right\},
$$




$$
\overline{\mathfrak{M}^{+} \xi_{0}}=\left\{\xi \in \mathscr{H}:\langle\xi, \eta\rangle \geq 0 \text { for all } \eta \in \overline{\mathfrak{M}^{+} \xi_{0}}\right\} .
$$

By these results and the Tomita-Takesaki theorem we obtain $J x^{*} x \xi_{0}=$ $J x^{*} J^{2} x J \xi_{0}=x^{\prime *} x^{\prime} \xi_{0} \in \mathfrak{M}^{\prime+} \xi_{0}$ for some $x^{\prime} \in \mathfrak{M}^{\prime}$, hence we get $J \overline{\mathfrak{M}^{+} \xi_{0}}=$ $\overline{\mathfrak{M}^{\prime+} \xi_{0}}$. Let $x \in \mathfrak{M}$ and put $y=J x^{\prime} J \in \mathfrak{M}$ for some $x^{\prime} \in \mathfrak{M}^{\prime} ;$ then by the assumption

$$
\begin{aligned}
\left\langle\hat{T}^{*}\left(x^{*} x \xi_{0}\right), x^{* *} x^{\prime} \xi_{0}\right\rangle & =\left\langle\hat{T}^{*}\left(x^{*} x\right) \xi_{0}, J y^{*} y \xi_{0}\right\rangle \\
& =\left\langle x^{*} x \xi_{0}, J \hat{T}\left(y^{*} y \xi_{0}\right)\right\rangle \\
& =\left\langle x^{*} x \xi_{0}, \eta\right\rangle \\
& \geq 0
\end{aligned}
$$

for all $x^{\prime} \in \mathfrak{M}^{\prime}$, where $\eta=J \hat{T}\left(y^{*} y \xi_{0}\right) \in \overline{\mathfrak{M}^{+} \xi_{0}}$, hence $\hat{T}^{*}\left(x^{*} x \xi_{0}\right) \in \overline{\mathfrak{M}^{+} \xi_{0}}$ and we conclude.

4. $\Rightarrow 5$. Assuming 4. we have

$$
0 \leq\left\langle\hat{T}^{*}\left(x^{*} x \xi_{0}\right), x^{\prime *} x^{\prime} \xi_{0}\right\rangle=\left\langle x^{*} x \xi_{0}, \hat{T}\left(x^{\prime *} x^{\prime} \xi_{0}\right)\right\rangle
$$

for all $x \in \mathfrak{M}$ and $x^{\prime} \in \mathfrak{M}^{\prime}$, hence $\hat{T}\left(\mathfrak{M}^{+} \xi_{0}\right) \subseteq \overline{\mathfrak{M}^{\prime+} \xi_{0}}$.

5. $\Rightarrow$ 6. Define $\psi_{x}: \mathfrak{M}^{\prime} \longrightarrow \mathbb{C}$ by $\psi_{x}(y)=\left\langle x \xi_{0}, \hat{T}\left(y \xi_{0}\right)\right\rangle$ for a fixed $x \in \mathfrak{M}^{+}$. By $(2.40)$ we have $\psi_{x}(y) \geq 0$ for $y \in \mathfrak{M}^{\prime+}$, so $\psi_{x}$ is a positive normal linear functional on $\mathfrak{M}^{\prime}$. Now let $y \in \mathfrak{M}^{\prime+}$ and choose a net $\left\{y_{i}\right\}_{i \in I} \subseteq \mathfrak{M}^{\prime+}$ such that $\lim _{i \rightarrow \infty} y_{i} \xi_{0}=\hat{T}\left(y \xi_{0}\right)$. Then since $\hat{T}\left(\xi_{0}\right)=\xi_{0}$,

$$
\begin{aligned}
\psi_{x}(y) & =\lim _{i \rightarrow \infty}\left\langle x \xi_{0}, y_{i} \xi_{0}\right\rangle=\lim _{i \rightarrow \infty}\left\langle x y_{i}^{1 / 2} \xi_{0}, y_{i}^{1 / 2} \xi_{0}\right\rangle \\
& \leq\|x\| \lim _{i \rightarrow \infty}\left\langle\xi_{0}, y_{i} \xi_{0}\right\rangle \\
& =\|x\|\left\langle\xi_{0}, \hat{T}\left(y \xi_{0}\right)\right\rangle \\
& =\|x\|\left\langle\xi_{0}, y \xi_{0}\right\rangle .
\end{aligned}
$$

So $\psi_{x} \leq\|x\|\left\langle\xi_{0}, \cdot \xi_{0}\right\rangle$ on $\mathfrak{M}^{++}$, hence by the Radon-Nikodym theorem there exists $a_{x} \in \mathfrak{M}^{\prime \prime+}=\mathfrak{M}^{+}$such that $\psi_{x}(y)=\left\langle a_{x} \xi_{0}, y \xi_{0}\right\rangle$ for all $y \in \mathfrak{M}^{\prime}$. Now for each $x \in \mathfrak{M}^{+}$define $T^{\beta}(x)=a_{x} \in \mathfrak{M}^{+}$and extend to a positive linear map $T^{\beta} \in \mathrm{L}(\mathfrak{M})$. This map satisfies by construction

$$
\left\langle T^{\beta}(x) \xi_{0}, y \xi_{0}\right\rangle=\left\langle x \xi_{0}, \hat{T}\left(y \xi_{0}\right)\right\rangle \quad \text { for all } x \in \mathfrak{M}, y \in \mathfrak{M}^{\prime},
$$

hence it follows that

$$
\left\langle T^{\beta}(x) \xi_{0}, \xi\right\rangle=\left\langle x \xi_{0}, \hat{T}(\xi)\right\rangle \quad \text { for all } x \in \mathfrak{M}, \xi \in \mathscr{H} .
$$


In particular,

$$
\omega\left(T^{\beta}(x) y\right)=\left\langle\xi_{0}, T^{\beta}(x) y \xi_{0}\right\rangle=\left\langle\xi_{0}, x T(y) \xi_{0}\right\rangle=\omega(x T(y))
$$

for all $x, y \in \mathfrak{M}$, hence $T$ satisfies detailed balance II with respect to $\omega$.

6 . $\Rightarrow 2$. By assumption we have

$$
\left\langle T^{\beta}(x) \xi_{0}, y \xi_{0}\right\rangle=\left\langle x \xi_{0}, \hat{T}\left(y \xi_{0}\right)\right\rangle=\left\langle\hat{T}^{*}\left(x \xi_{0}\right), y \xi_{0}\right\rangle
$$

for any $x, y \in \mathfrak{M}$, in particular $T^{\beta}(x) \xi_{0}=\hat{T}^{*}\left(x \xi_{0}\right)$ for all $x \in \mathfrak{M}$. The positivity of $T^{\beta}$ implies that

$$
\begin{aligned}
\left\langle\hat{T}^{*}\left(S x \xi_{0}\right), y \xi_{0}\right\rangle & =\left\langle S T^{\beta}(x) \xi_{0}, y \xi_{0}\right\rangle=\left\langle F y \xi_{0}, T^{\beta}(x) \xi_{0}\right\rangle \\
& =\left\langle F y \xi_{0}, \hat{T}^{*}\left(x \xi_{0}\right)\right\rangle \\
& =\left\langle S \hat{T}^{*}\left(x \xi_{0}\right), y \xi_{0}\right\rangle
\end{aligned}
$$

i. e. $\hat{T}^{*} S\left(x \xi_{0}\right)=S \hat{T}^{*}\left(x \xi_{0}\right)$. Since $\mathfrak{M} \xi_{0}$ is a core for $S$ it follows that $S$ and $\hat{T}^{*}$ commute strongly, in particular, they commute on dom $\Delta^{1 / 2}=$ $\operatorname{dom} S$ and we have $\hat{T}^{*}\left(\operatorname{dom} \Delta^{1 / 2}\right) \subseteq \operatorname{dom} \Delta^{1 / 2}$. Moreover, $S$ and $\hat{T}$ commute strongly. Now by the inclusion $J \operatorname{dom} \Delta^{1 / 2} \subseteq \operatorname{dom} \Delta^{-1 / 2}$ it follows for any $\eta \in \operatorname{dom} \Delta^{-1 / 2}$ and $\xi \in \operatorname{dom} \Delta^{1 / 2}$ that the map

$$
\begin{aligned}
\xi \mapsto\left\langle J \hat{T}(\eta), \Delta^{1 / 2} \xi\right\rangle & =\left\langle J \Delta^{1 / 2} \xi, \hat{T}(\eta)\right\rangle \\
& =\left\langle\hat{T}^{*}(S \xi), \eta\right\rangle \\
& =\left\langle S \hat{T}^{*}(\xi), \eta\right\rangle \\
& =\langle\hat{T}(F \eta), \xi\rangle
\end{aligned}
$$

is bounded and hence $J \hat{T}(\eta) \in \operatorname{dom} \Delta^{1 / 2}$, i. e. we have the inclusion $\hat{T}\left(\operatorname{dom} \Delta^{-1 / 2}\right) \subseteq \operatorname{dom} \Delta^{-1 / 2}$. By transposition of $\hat{T}^{*} S\left(x \xi_{0}\right)=S \hat{T}^{*}\left(x \xi_{0}\right)$ we obtain for $\xi \in \operatorname{dom} \Delta^{1 / 2}$ and $\eta \in \operatorname{dom} \Delta^{-1 / 2}$ that

$$
\begin{aligned}
\left\langle\eta, \hat{T}^{*}\left(J \Delta^{1 / 2} \xi\right)\right\rangle & =\left\langle\xi, \Delta^{1 / 2} J \hat{T}(\eta)\right\rangle \\
& =\left\langle\eta, J \Delta^{1 / 2} \hat{T}^{*}(\xi)\right\rangle \\
& =\left\langle\xi, \hat{T}\left(\Delta^{1 / 2} J \eta\right)\right\rangle
\end{aligned}
$$

i. e. $\hat{T}$ and $\Delta^{1 / 2} J$ commute strongly. Then it follows that

$$
\begin{aligned}
\hat{T}(\Delta \xi) & =\hat{T}\left(\Delta^{1 / 2} J^{2} \Delta^{1 / 2} \xi\right) \\
& =\Delta^{1 / 2} J \hat{T}\left(J \Delta^{1 / 2} \xi\right)
\end{aligned}
$$




$$
\begin{aligned}
& =\Delta^{1 / 2} J^{2} \Delta^{1 / 2} \hat{T}(\xi) \\
& =\Delta \hat{T}(\xi)
\end{aligned}
$$

for any $\xi \in \operatorname{dom} \Delta$, hence $\hat{T}$ and $\Delta$ commute strongly.

We now show the normality of $T^{\beta}$. In (2.41) we put $y=a^{*} b$ for $a, b \in$ $\mathfrak{M}^{\prime}$, then we infer

$$
\left\langle T^{\beta}(x) a \xi_{0}, b \xi_{0}\right\rangle=\left\langle x \xi_{0}, \hat{T}\left(a^{*} b \xi_{0}\right)\right\rangle \quad \text { for all } a, b \in \mathfrak{M}^{\prime}, x \in \mathfrak{M} .
$$

If $\left\{x_{i}\right\}_{i \in I} \subseteq$ ball $\mathfrak{M}$ is a net with $\lim x_{i}=x$ in the ultraweak topology it follows from the last equation that $\lim \left\langle T^{\beta}\left(x_{i}\right) a \xi_{0}, b \xi_{0}\right\rangle=\left\langle T^{\beta}(x) a \xi_{0}, b \xi_{0}\right\rangle$. Now let $\eta, \xi \in \mathscr{H}$ and $\epsilon>0$. Choose $a, b \in \mathfrak{M}^{\prime}$ such that $\left\|a \xi_{0}-\eta\right\|<\epsilon$ and $\left\|b \xi_{0}-\xi\right\|<\epsilon$, then since $T^{\beta}$ is a contractive, positive and unital map we have

$$
\begin{aligned}
\mid\left\langle\left( T^{\beta}\left(x_{i}\right)-\right.\right. & \left.T^{\beta}(x) \eta, \xi\right\rangle \mid \\
\leq \mid & \left\langle T^{\beta}\left(x_{i}-x\right) \eta, \xi\right\rangle-\left\langle T^{\beta}\left(x_{i}-x\right) a \xi_{0}, \xi\right\rangle \mid \\
& \quad+\left|\left\langle T^{\beta}\left(x_{i}-x\right) a \xi_{0}, \xi\right\rangle-\left\langle T^{\beta}\left(x_{i}-x\right) a \xi_{0}, b \xi_{0}\right\rangle\right| \\
& \quad+\left|\left\langle T^{\beta}\left(x_{i}-x\right) a \xi_{0}, b \xi_{0}\right\rangle\right| \\
\leq & \|\xi\| \cdot\left\|x_{i}-x\right\| \cdot\left\|\eta-a \xi_{0}\right\|+\left\|a \xi_{0}\right\| \cdot\left\|x_{i}-x\right\| \cdot\left\|\xi-b \xi_{0}\right\| \\
& \quad+\left|\left\langle T^{\beta}\left(x_{i}-x\right) a \xi_{0}, b \xi_{0}\right\rangle\right| \\
\leq 2 \epsilon \| & \|+2 \epsilon(\|\eta\|+\epsilon)+\left|\left\langle T^{\beta}\left(x_{i}-x\right) a \xi_{0}, b \xi_{0}\right\rangle\right|,
\end{aligned}
$$

hence $\lim T^{\beta}\left(x_{i}\right)=T^{\beta}(x)$. We have thus shown that the restriction of $T^{\beta}$ to ball $\mathfrak{M}$ is weak-operator continuous; by an application of the KreinŠmulian theorem, using the fact that the weak operator topology and the ultraweak topology agree on ball $\mathfrak{M}$, we conclude that $T^{\beta}$ is ultraweakly continuous, hence normal.

Remark 2.30 The above proof shows that if two contractions $T$ and $T^{\beta}$ in $\mathrm{L}(\mathfrak{M})$ are related by $(2.33)$ where $\omega$ is a faithful normal state on $\mathfrak{M}$ then $T^{\beta}$ is normal provided $T$ is normal, without assuming any positivity properties of $T$ and $T^{\beta}$.

Corollary 2.31 Let $\mathfrak{M}$ be a commutative von Neumann algebra, $T \in$ $\mathrm{L}(\mathfrak{M})$ a normal positive map, and $\omega$ a normal state on $\mathfrak{M}$ such that $\omega \circ T \leq$ $\omega$. Then $T$ satisfies detailed balance II with respect to $\omega$.

Proof. By commutativity of $\mathfrak{M}$, the map $T$ is completely positive and hence satisfies Kadison's inequality, thus there exists a contraction $\hat{T}$ on $\mathscr{H}$ 
such that $\hat{T}\left(x \xi_{0}\right)=T(x) \xi_{0}$ for all $x \in \mathfrak{M}$. Since $\sigma_{t}^{\omega}=\operatorname{id}_{\mathfrak{M}}$ condition 2 . of Theorem 2.29 is satisfied and we conclude that $T$ satisfies detailed balance II.

Proposition 2.32 Let $\mathfrak{M}$ be a von Neumann algebra acting on $\mathscr{H}, \omega$ a faithful normal state on $\mathfrak{M}$ and $\left\{T_{t}\right\}_{t \geq 0}$ an ultraweakly continuous semigroup of positive unital maps on $\mathfrak{M}$ with generator $Z$. Then $\left\{T_{t}\right\}_{t \geq 0}$ satisfies detailed balance II with respect to $\omega$ if and only if there exists a generator $Z^{\beta}$ of an ultraweakly continuous semigroup of positive unital maps such that

$$
\omega\left(Z^{\beta}(x) y\right)=\omega(x Z(y)) \quad \text { for all } x \in \operatorname{dom} Z^{\beta}, y \in \operatorname{dom} Z \text {. }
$$

Proof. Assume that a generator $Z^{\beta}$ satisfying (2.42) exists. Then for any $\lambda \in \mathbb{C}$ with $\operatorname{Re} \lambda>0$ we have

$$
\begin{aligned}
\omega\left[\left(R_{\lambda}\left(Z^{\beta}\right) x\right) y\right] & =\omega\left[\left(R_{\lambda}\left(Z^{\beta}\right) x\right)(\lambda \mathbb{1}+Z) R_{\lambda}(Z) y\right] \\
& =\omega\left[\left(\left(\lambda \mathbb{1}+Z^{\beta}\right) R_{\lambda}\left(Z^{\beta}\right) x\right) R_{\lambda}(Z) y\right] \\
& =\omega\left[x R_{\lambda}(Z) y\right]
\end{aligned}
$$

for all $x, y \in \mathfrak{M}$. Now using $R_{\lambda}(Z) x=\int_{0}^{\infty} \mathrm{e}^{-\lambda t} T_{t}(x) \mathrm{d} t$ and $R_{\lambda}\left(Z^{\beta}\right) x=$ $\int_{0}^{\infty} \mathrm{e}^{-\lambda t} T_{t}^{\beta}(x) \mathrm{d} t$, where $T_{t}^{\beta}=\mathrm{e}^{t Z}$, we obtain by normality of $\omega$

$$
\int_{0}^{\infty} \mathrm{e}^{-\lambda t} \omega\left(T_{t}^{\beta}(x) y\right) \mathrm{d} t=\int_{0}^{\infty} \mathrm{e}^{-\lambda t} \omega\left(x T_{t}(y)\right) \mathrm{d} t
$$

for all $x, y \in \mathfrak{M}$ and $\lambda \in \mathbb{C}$ with $\operatorname{Re} \lambda>0$. Since the maps $t \mapsto \omega\left(T_{t}^{\beta}(x) y\right)$ and $t \mapsto \omega\left(x T_{t}(y)\right)$ are continuous we obtain by general properties of the Laplace transform that $\omega\left(T_{t}^{\beta}(x) y\right)=\omega\left(x T_{t}(y)\right)$, i. e. detailed balance II.

Conversely, from the detailed balance condition (2.34) we get

$$
\frac{1}{t} \omega\left(\left(T_{t}^{\beta}(x)-x\right) y\right)=\frac{1}{t} \omega\left(x\left(T_{t}(y)-y\right)\right),
$$

and if $x \in \operatorname{dom} Z^{\beta}$ and $y \in \operatorname{dom} Z$ we obtain (2.42) upon letting $t \downarrow 0$.

The next result provides an alternative characterization of the subalgebra $\mathfrak{M}_{1}$, see (2.9), of the isometric-sweeping decomposition.

Proposition 2.33 Suppose $\left\{T_{t}\right\}_{t \geq 0}$ is an ultraweakly continuous semigroup of strongly positive unital maps on a von Neumann algebra $\mathfrak{M}$ such that $\left\{T_{t}\right\}_{t \geq 0}$ satisfies detailed balance II with respect to a faithful normal state $\omega$ on $\mathfrak{M}$. Then $\mathfrak{M}_{1}$, defined in (2.9), is given by

$$
\mathfrak{M}_{1}=\left\{x \in \mathfrak{M}: T_{t}^{\beta} \circ T_{t}(x)=x \text { for all } t \geq 0\right\} .
$$


Proof. First it is clear that $\omega$ is invariant under $\left\{T_{t}\right\}_{t \geq 0}$, hence Corollary 2.5 applies. Let $x \in \mathfrak{M}_{1}$, hence $x \in M$, i. e. $T_{t}(x y)=T_{t}(x) T_{t}(y)$ for all $y \in \mathfrak{M}$ and $t \geq 0$ by (2.11). Now

$$
\begin{aligned}
\omega\left(x^{*} y\right) & =\omega\left(T_{t}\left(x^{*} y\right)\right)=\omega\left(T_{t}(x) T_{t}(y)\right) \\
& =\omega\left(T_{t}^{\beta} \circ T_{t}(x)^{*} y\right)
\end{aligned}
$$

for any $y \in \mathfrak{M}$. As in Lemma 2.22 we conclude $T_{t}^{\beta} \circ T_{t}(x)=x$ for any $t \geq 0$. Conversely, suppose that $x \in \mathfrak{M}$ satisfies $T_{t}^{\beta} \circ T_{t}(x)=x$ for any $t \geq 0$. Then by a similar calculation as above we find $\omega\left(x^{*} x-T_{t}(x) T_{t}(x)^{*}\right)=0$ for any $t \geq 0$, hence $x \in M$ by (2.12). Using positivity of $T_{t}$ and $T_{t}^{\beta}$ we find that $T_{t}^{\beta} \circ T_{t}(x)^{*}=x^{*}$, and consequently also $x \in M^{*}$ by (2.14), hence $x \in \mathfrak{M}_{1}=M \cap M^{*}$.

Corollary 2.34 Suppose that $\left\{T_{t}\right\}_{t \geq 0}$ is an ultraweakly continuous semigroup of strongly positive unital maps on a von Neumann algebra $\mathfrak{M}$ acting on $\mathscr{H}$ such that $\left\{T_{t}\right\}_{t \geq 0}$ satisfies detailed balance II with respect to a faithful normal state $\omega$ on $\mathfrak{M}$. Then the conclusion of Theorem 2.3 holds.

Proof. By Proposition 2.23 there exist contractions $\hat{T}_{t}$ on $\mathscr{H}$ such that $\hat{T}_{t}\left(x \xi_{0}\right)=T_{t}(x) \xi_{0}$, where $x \in \mathfrak{M}$, and where $\xi_{0} \in \mathscr{H}$ is the unit vector corresponding to $\omega$ (without loss of generality we assume that $\omega$ is a vector state). Then Theorem 2.29 applies and we see that $\left[T_{t}, \sigma_{s}^{\omega}\right]=0$ for any $t \geq 0$ and $s \in \mathbb{R}$. Thus the conditions of Theorem 2.3 are satisfied.

\subsubsection{Sufficient Conditions for Detailed Balance}

In this section we give a condition for a linear map to satisfy detailed balance II with respect to some state. The result states that the existence of a certain kind of dilation is sufficient for detailed balance to hold.

Let $\mathfrak{M}$ be a von Neumann algebra and $T$ a normal contractive linear map on $\mathfrak{M}$, and let $\omega$ be a normal state on $\mathfrak{M}$ such that $\omega \circ T=\omega$. A dilation (of first order) of $(T, \omega)$ is a quintuple $(\mathfrak{N}, \varphi, \alpha, i, E)$ consisting of a von Neumann algebra $\mathfrak{N}$, a faithful normal state $\varphi$ on $\mathfrak{N}$, an automorphism $\alpha \in$ Aut $\mathfrak{N}$, a normal injective ${ }^{*}$-homomorphism $i: \mathfrak{M} \longrightarrow \mathfrak{N}$ (an embedding of $\mathfrak{M}$ in $\mathfrak{N}$ ), and a normal linear map $E: \mathfrak{N} \longrightarrow \mathfrak{M}$ such that

$$
\begin{aligned}
\omega \circ E & =\varphi, & \varphi \circ i & =\omega, \\
E \circ i & =\operatorname{id}_{\mathfrak{M}}, & \varphi \circ \alpha & =\varphi,
\end{aligned}
$$


and such that the diagram

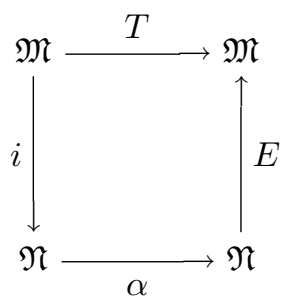

commutes, i. e. $T=E \circ \alpha \circ i$.

Proposition 2.35 Suppose that the pair $(T, \omega)$ has a dilation of first or$\operatorname{der}(\mathfrak{N}, \varphi, \alpha, i, E)$. Then $T$ satisfies detailed balance II with respect to $\omega$ and $T^{\beta}$ is given by

$$
T^{\beta}=E \circ \alpha^{-1} \circ i \text {. }
$$

Proof. Observe that $i(\mathfrak{M}) \subseteq \mathfrak{N}$ is a von Neumann subalgebra. We consider the modular group $\left\{\sigma_{t}^{\varphi}\right\}_{t \in \mathbb{R}} \subseteq$ Aut $\mathfrak{N}$ with respect to the faithful normal state $\varphi$ and the modular group $\left\{\sigma_{t}^{\omega}\right\}_{t \in \mathbb{R}} \subseteq$ Aut $\mathfrak{M}$ with respect to $\omega$. Since $\mathfrak{M}$ and $i(\mathfrak{M})$ are ${ }^{*}$-isomorphic and since $\varphi \circ i=\omega$, it follows that $i$ intertwines between the two modular groups, $\sigma_{t}^{\varphi} \circ i=i \circ \sigma_{t}^{\omega}$ in view of the uniqueness of the modular group on $i(\mathfrak{M}) \cong \mathfrak{M}$. In particular, $i(\mathfrak{M}) \subseteq \mathfrak{N}$ is invariant under the modular group $\left\{\sigma_{t}^{\varphi}\right\}_{t \in \mathbb{R}}$, thus by Takesaki's theorem, Theorem 2.2, there exists a faithful normal conditional expectation $E_{0}$ on $\mathfrak{N}$ onto $i(\mathfrak{M})$ such that $\varphi \circ E_{0}=\varphi$. Since $E_{0}$ is uniquely determined by this condition and since $\varphi \circ i \circ E=\omega \circ E=\varphi$ we obtain $i \circ E=$ $E_{0}$. Therefore, if $x, y \in \mathfrak{M}$, by what was established above and by the properties of conditional expectation,

$$
\begin{aligned}
\omega(x T(y)) & =\varphi(i(x) i(T(y))) \\
& =\varphi(i(x) i[(E \circ \alpha \circ i)(y)]) \\
& =\varphi\left(i(x)\left(E_{0} \circ \alpha \circ E_{0}\right)(i(y))\right) \\
& =\varphi\left(i(x) E_{0}\left[\left(\alpha \circ E_{0} \circ i\right)(y)\right]\right) \\
& =\varphi\left(E_{0}\left[i(x)\left(\alpha \circ E_{0} \circ i\right)(y)\right]\right) \\
& =\varphi\left(i(x)\left(\alpha \circ E_{0} \circ i\right)(y)\right) \\
& =\varphi\left(\alpha^{-1}(i(x)) E_{0}(i(y))\right) \\
& =\varphi\left(E_{0}\left(\alpha^{-1}(i(x))\right) i(y)\right) \\
& =\varphi\left(\left(i \circ E \circ \alpha^{-1} \circ i\right)(x) i(y)\right)
\end{aligned}
$$




$$
\begin{aligned}
& =\omega\left(\left(E \circ \alpha^{-1} \circ i\right)(x) y\right) \\
& =\omega\left(T^{\beta}(x) y\right),
\end{aligned}
$$

which shows (2.44).

\subsubsection{Detailed Balance and Approach to Equilibrium}

The characterization of detailed balance in Theorem 2.29 and the isometric-sweeping decomposition in Theorem 2.3 enable us to prove a main result from [98] concerning the return to equilibrium for a quantum dynamical semigroup under weaker assumptions.

Proposition 2.36 Let $\mathfrak{M}$ be a von Neumann algebra, $\omega$ a faithful normal state on $\mathfrak{M}$ and $\left\{T_{t}\right\}_{t \geq 0}$ an ultraweakly continuous semigroup of strongly positive unital maps. Suppose that the following conditions are satisfied:

1. $\left\{T_{t}\right\}_{t \geq 0}$ satisfies detailed balance II with respect to $\omega$.

2. Suppose there is a ${ }^{*}$-invariant dense subspace $M_{0} \subseteq \mathfrak{M}$ such that for any $x \in M_{0}$ and $y \in \mathfrak{M}_{1}$ the limit

$$
\lim _{t \rightarrow \infty} \omega\left(x T_{t}(y)\right)
$$

exists. Here $\mathfrak{M}_{1}$ is given by (2.9).

Let $E_{0}$ be the normal conditional expectation onto fix $\left\{T_{t}\right\}_{t \geq 0}$ (which exists by Corollary 1.27). Then the limit

$$
\lim _{t \rightarrow \infty} T_{t}(x)=E_{0}(x)
$$

exists relative to the ultraweak topology for any $x \in \mathfrak{M}$.

Proof. We can suppose without loss of generality that $\omega(x)=\left\langle\xi_{0}, x \xi_{0}\right\rangle$ for a cyclic and separating vector $\xi_{0} \in \mathscr{H}$. It is easy to see that $\left(M_{0} \xi_{0}\right)^{\perp}=$ $\{0\}$, hence $M_{0} \xi_{0} \subseteq \mathscr{H}$ is dense. By assumption (2.45) the limit

$$
\lim _{t \rightarrow \infty}\left\langle x \xi_{0}, T_{t}(y) \xi_{0}\right\rangle
$$

exists for all $x \in M_{0}$ and $y \in \mathfrak{M}_{1}$, hence also

$$
\lim _{t \rightarrow \infty}\left\langle\xi, T_{t}(y) \xi_{0}\right\rangle
$$

exists for all $\xi \in \mathscr{H}$ and $y \in \mathfrak{M}_{1}$. In particular, for $\xi=a b^{*} \xi_{0}, a, b \in \mathfrak{M}^{\prime}$, it follows that

$$
\lim _{t \rightarrow \infty}\left\langle a \xi_{0}, T_{t}(y) b \xi_{0}\right\rangle
$$


exists and hence

$$
\lim _{t \rightarrow \infty}\left\langle\eta, T_{t}(y) \xi\right\rangle
$$

exists for all $\eta, \xi \in \mathscr{H}$, which, by contractivity of $T_{t}$, implies that the limit $\lim _{t \rightarrow \infty} T_{t}(y)$ exists ultraweakly for any $y \in \mathfrak{M}_{1}$. Now let $x \in \mathfrak{M}$ and $\varphi \in \mathfrak{M}_{*}$, then if $E$ is the conditional expectation onto $\mathfrak{M}_{1}$ we obtain by the properties of $\mathfrak{M}_{2}$ that

$$
\begin{aligned}
\lim _{t \rightarrow \infty} \varphi\left(T_{t}(x)\right) & =\lim _{t \rightarrow \infty} \varphi\left(T_{t}(E x)\right)+\lim _{t \rightarrow \infty} \varphi\left(T_{t}\left(E^{\perp} x\right)\right) \\
& =\lim _{t \rightarrow \infty} \varphi\left(E T_{t}(x)\right)
\end{aligned}
$$

hence $P(x):=\lim _{t \rightarrow \infty} T_{t}(x)$ exists ultraweakly for any $x \in \mathfrak{M}$. Clearly, $P(x) \in \operatorname{fix}\left\{T_{t}\right\}_{t \geq 0}$ and we see that $P$ is a projection onto fix $\left\{T_{t}\right\}_{t \geq 0}$ such that $\omega \circ P=\omega$ and $\|P\| \leq 1$, and since $P(\mathbb{1})=\mathbb{1}$ we see that $P$ must be a conditional expectation by Tomiyama's theorem. By Corollary 1.27 there exists a normal conditional expectation $E_{0}$ onto fix $\left\{T_{t}\right\}_{t \geq 0}$ and by Corollary 1.32 we have $\omega \circ E_{0}=\omega$. Since a conditional expectation is uniquely determined by this property (see [4]) we conclude $P=E_{0}$.

Corollary 2.37 Under the assumptions of Proposition 2.36, if we have for the fixed point algebra fix $\left\{T_{t}\right\}_{t \geq 0}=\mathbb{C} \mathbb{1}$ then $E_{0}(x)=\mathbb{1} \omega(x)$, i. e. for any normal state $\varphi$ on $\mathfrak{M}$ it follows that

$$
\lim _{t \rightarrow \infty}\left\langle T_{t}(x), \varphi\right\rangle=\omega(x), \quad x \in \mathfrak{M}
$$

Proof. Let $x \in \mathfrak{M}$, then $\left\langle T_{t}(x), \omega\right\rangle \rightarrow\left\langle E_{0}(x), \omega\right\rangle=\omega(x)$ as $t \rightarrow \infty$, i. e. $E_{0}(x)=\mathbb{1} \omega(x)$, and the last assertion follows.

Thus in this situation the semigroup $\left\{T_{t}\right\}_{t \geq 0}$ describes the return to equilibrium of a physical system. 


\section{Chapter 3.}

\section{Decoherence: Physical Interpretation}

In this chapter we give a mathematically precise notion of decoherence in the algebraic formulation of (quantum) physics. This notion introduces the concept of the algebra of effective observables, which describes the system after decoherence has taken place. By using the results of Chapter 2 we are able to give a number of criteria which ensure that an open system with an irreversible time evolution given by a continuous semigroup of normal positive maps on a von Neumann algebra displays decoherence. Finally, we present some results concerning the structure of the effective algebra and briefly discuss the possible scenarios of decoherence, and mention the utility of decoherence in the discussion of quantum measurement processes.

\subsection{Decoherence in the Algebraic Formulation}

\subsubsection{Introduction}

Quantum and classical physics are different in many ways. Not only are its usual elementary textbook formulations, using Hilbert spaces and linear operators on the one hand and phase space and probability distributions on it on the other, apparently at variance, but quantum theory has number of peculiar properties which are absent in classical physics. For example, there is the superposition principle which states that any linear combination of two pure states (Hilbert space vectors) of a quantum system is again an admissible state. Moreover, the role of measurement seems to be much more involved in quantum theory for there seems to be no way to observe a quantum system without perturbing it. This is related to the uncertainty principle which leads to the absence of dispersion free states 
in quantum theory, compare Section 1.2.4. These fundamental differences raise the question of the relation of classical and quantum physics. Clearly, in view of the above mentioned formal and conceptual differences it is not clear in which way quantum mechanics could be thought of a generalization of classical mechanics, or in which limit the former becomes the latter. Indeed, between these two theories there is no relation as simple as between Newtonian and relativistic kinematics and mechanics, where the transition is governed by a scale parameter, the light speed $c$. Quantum mechanics contains no such parameter which would inhibit, for example, the superposition principle and make a smooth transition possible. For this reason, the Copenhagen interpretation of quantum mechanics, advocated by Bohr, introduced the concept of complementarity to describe this fundamental discrepancy. It was contended by Bohr that the classical and quantum description of a physical system are complementary, and that the quantum description must not be applied to classical systems, and vice versa, to avoid paradoxes. The most prominent paradox which arises from the superposition principle when applied to classical (macroscopic) systems is exemplified by the well-known Schrödinger's cat paradox. Here, a cat is steered by a suitably engineered interaction with an atomic system into a superposition between the states "dead" and "alive", so that according to the quantum rules the status of its vital functions is indeterminate, in conflict with observation. Similar paradoxical superpositions between macroscopically different states can be created in analogous thought experiments. For example, measurement-like interactions between a quantum system and an instrument naturally lead to superpositions of different pointer positions. However, the dial is a macroscopic system and nonclassical superposition of different dial readings have never been observed. What is more, according to Bohr, this "classicality" is a necessity to interpret quantum physics, for the very method of physics and natural science in general relies on the observation and description of unambiguous and objective "facts". In the Copenhagen approach they are generated by the reduction or collapse of the quantum state and play the role of the link between the quantum and classical worlds. This leaves us in an embarrassing situation: we have two very distinct physical theories with apparently little logical relation, but which are both necessary for a complete description of nature. Moreover, we have no way to decide when a physical system is quantum or classical; neither classical nor quantum physics contain an intrinsic element which would limit their ranges of applicability. Even though the Copenhagen interpretation was and still is of much pragmatic value in physics, it begs the question.

A way out is suggested by the program of environmental decoherence. 
It contends that at a fundamental level the world is a quantum world, but that most systems, especially macroscopic systems, strongly interact with their environment. Clearly, the objects around us, for example, scatter air molecules and photons, and due to this interaction with their environment, they are open systems. Decoherence claims that due to this unavoidable openness the systems can dynamically acquire classical properties and effectively behave like classical systems. A simple example is a macroscopic particle which scatters air molecules and photons. Heuristically, due to this interaction information about its position is carried away into the environment and an observer can infer from this information the particle's position, hence we can guess that we are dealing with a measurement-like interaction. Accordingly, by the Lüders-von Neumann reduction postulate, the particle will be found in a position eigenstate. In this sense the environment continuously monitors the particle's position which thereby becomes classical. Thus it is expected that a macroscopic particle is never found in a coherent superposition of different position eigenstates - position has become a classical variable. More generally, the measurement-like interaction between a system and its environment leads to a strong entanglement between them which effectively destroys phase relations between superpositions of vectors from certain subspaces of the Hilbert space of the system, thus limiting the validity of the superposition principle. So we have reason to suspect that the openness of a quantum system may lead to a dynamical emergence of superselection rules - in the above example between the different position eigenstates.

It is difficult to say where these ideas were first spelled out. An early contribution along these lines is the paper [134] by Zeh. Decoherence was popularized by Zurek's articles $[135,136]$. Since then, a huge amount of literature has been generated, see the monograph [15] for an extensive bibliography as well as [5]. The above example of localization of a mass point by scattering particles of its environment has first been treated by Joos and Zeh [82]. They consider a test particle subject to scattering by other particles in its environment, neglecting recoil (i.e. the back-reaction of the environment on the system). Let $|x\rangle$ denote the (generalized) position eigenvector of the test particle and $|\chi\rangle$ the state of an incoming particle. Neglecting recoil this will lead to a time evolution $|x\rangle \otimes|\chi\rangle \stackrel{t}{\rightarrow}|x\rangle \otimes S_{x}|\chi\rangle$, where $S_{x}|\chi\rangle$ is the scattered state, calculated by using an appropriate scattering matrix. For a wave packet described by a wave function $\xi$ one obtains $\int \xi(x)|x\rangle \otimes|\chi\rangle \mathrm{d} x \stackrel{t}{\rightarrow} \int \xi(x)|x\rangle \otimes S_{x}|\chi\rangle \mathrm{d} x$, leading to the reduced density matrix elements $\rho\left(x, x^{\prime}, t\right)=\left\langle x|\rho(t)| x^{\prime}\right\rangle=\xi(x) \bar{\xi}\left(x^{\prime}\right)\left\langle\chi\left|S_{x^{\prime}}^{*} S_{x}\right| \chi\right\rangle$, where $\rho(t)$ denotes the reduced density matrix of the test particle. If the 
wavelength $\lambda$ of the scattered particle satisfies $\lambda \gg\left|x-x^{\prime}\right|$, i. e. a single scattering event does not resolve the distance $\left|x-x^{\prime}\right|$, and the scattering interaction is translationally invariant, the expression $\left\langle\chi\left|S_{x^{\prime}}^{*} S_{x}\right| \chi\right\rangle$ can be calculated for incoming plane waves with wave number $k$, and adding contributions from many independent and individually ineffective scattering events leads to

$$
\rho\left(x, x^{\prime}, t\right)=\rho\left(x, x^{\prime}, 0\right) e^{-\Lambda\left(x-x^{\prime}\right)^{2} t} .
$$

The quantity

$$
\Lambda=\frac{k^{2} N v \sigma_{\mathrm{eff}}}{V}
$$

is called the localization rate, here $N v / V$ is the flux of incoming particles and $\sigma_{\text {eff }}$ is a quantity of the order of the total cross section. Thus we see that the diagonal elements $\rho\left(x, x^{\prime}, t\right)$ of the reduced density matrix in the position basis vanish effectively if $t \gg \Lambda\left|x-x^{\prime}\right|$, leading to localization of the test particle. The localization rate $\Lambda$ has been calculated for a number of different sizes of test particles and scattering particles [82], for example one finds for a dust particle (of a linear dimension of the order $10^{-3} \mathrm{~cm}$ ) a localization rate of $10^{6} \mathrm{~cm}^{-2} \mathrm{~s}^{-1}$ for cosmic background radiation; for air it is as large as $10^{36} \mathrm{~cm}^{-2} \mathrm{~s}^{-1}$. This shows that decoherence is a very effective process acting on a very short time scale. A number of other models for particle localization by scattering have been introduced, see [79] for some references. Moreover, we refer to the quoted literature for other applications and models of decoherence as well as for experimental tests.

\subsubsection{The Notion Decoherence in Algebraic Formulation}

We have seen in Section 1.1 that there exists a common mathematical framework for both quantum and classical systems, the algebraic framework. Not surprisingly it turns out to be very useful if we want to discuss the emergence of classical properties in quantum systems. Indeed, in this way we have already overcome a first obstacle, namely the discrepancy between the formalisms of classical and quantum physics mentioned in Section 3.1.1. Moreover, if we want to discuss decoherence in infinite systems rigorously we do not have much choice but to use the algebraic framework.

In this section we will define the notion of decoherence in the algebraic framework. As usual in mathematical physics, when definitions of concepts directly related to physical phenomena are given, the term "definition" should not be taken too literally. It rather means "working hypoth- 
esis" and one should be open minded to adapt it whenever the physical phenomena call for it.

A notion of decoherence in the algebraic framework was first introduced into the literature in [46], after it was introduced and studied in a special case in [105] and [106]. Following [46] we will now introduce the notion of decoherence which underlies the present work. We base our considerations on the setup of Section 1.3.2. Consider an open system described by a von Neumann algebra $\mathfrak{M}$ together with a irreversible (reduced) dynamics $\left\{T_{t}\right\}_{t \geq 0}$, which we will take here as a family of positive normal and unital maps such that $\mathbb{R}^{+} \ni t \mapsto T_{t}(x)$ is ultraweakly continuous for every $x \in \mathfrak{M}$.

Definition 3.1 We say that decoherence takes place (or that $\left\{T_{t}\right\}_{t \geq 0}$ displays decoherence) if the following holds true: There exists a von Neumann subalgebra $\mathfrak{M}_{1} \subseteq \mathfrak{M}$ and an ultraweakly continuous group of *automorphisms $\left\{\alpha_{t}\right\}_{t \in \mathbb{R}} \subseteq$ Aut $\mathfrak{M}_{1}$ such that $T_{t}\left(\mathfrak{M}_{1}\right) \subseteq \mathfrak{M}_{1}$ for any $t \geq 0$ and $T_{t}\left\lceil\mathfrak{M}_{1}=\alpha_{t}\right.$ for $t \geq 0$, and $a{ }^{*}$-invariant ultraweakly closed subspace $\mathfrak{M}_{2} \subseteq \mathfrak{M}$ with $T_{t}\left(\mathfrak{M}_{2}\right) \subseteq \mathfrak{M}_{2}$ for all $t \geq 0$ such that

$$
\mathfrak{M}=\mathfrak{M}_{1} \oplus \mathfrak{M}_{2},
$$

and such that

$$
\lim _{t \rightarrow \infty} T_{t}(x)=0, \quad x \in \mathfrak{M}_{2},
$$

in the ultraweak topology. Moreover, $\mathfrak{M}_{1}$ is chosen as the maximal von Neumann subalgebra on which $\left\{T_{t}\right\}_{t \geq 0}$ extends to a group of *automorphisms. We call $\mathfrak{M}_{1}$ the algebra of effective observables.

Notice that the direct sum in (3.3) is understood to be the linear algebraic direct sum. The physical interpretation of this definition is rather clear: Decoherence takes place if there is a von Neumann subalgebra on which the reduced dynamics $\left\{T_{t}\right\}_{t \geq 0}$ acts reversibly, i.e. as an automorphism group, and a complementary subspace with the property that the expectation values of all observables in $\mathfrak{M}_{2}$ become small as time becomes large because (3.4) means that

$$
\lim _{t \rightarrow \infty} \varphi\left(T_{t}(x)\right)=0
$$

for any $x \in \mathfrak{M}_{2}$ and any normal state $\varphi$ on $\mathfrak{M}$. Thus any observable $x=x^{*} \in \mathfrak{M}$ can be written as $x=x_{1}+x_{2}$, with $x_{i} \in \mathfrak{M}_{i}$ self-adjoint, $i=1,2$, where $x_{2}$ becomes effectively unobservable if we wait long enough. Thus after a sufficiently long time the system is effectively characterized by the algebra $\mathfrak{M}_{1}$ and the reversible time evolution given by the group $\left\{\alpha_{t}\right\}_{t \in \mathbb{R}}$, and thus effectively behaves like a closed system, but it may have properties different from the original one. 
Remark 3.2 According to Definition 3.1, if $\left\{T_{t}\right\}_{t \geq 0}$ is a group of automorphisms on $\mathfrak{M}$, i. e. reversible, then decoherence takes place and the splitting (3.3) is trivial, i. e. $\mathfrak{M}_{1}=\mathfrak{M}$ and $\mathfrak{M}_{2}=\{0\}$. However, we shall keep this slightly unfortunate terminology since it simplifies statements of some theorems, keeping in mind that physically decoherence corresponds to the case when $\mathfrak{M}_{2} \neq\{0\}$. This can only happen if $\left\{T_{t}\right\}_{t \geq 0}$ is not reversible.

Definition 3.1 does not make any requirements about the rate of convergence in (3.4). In many models we have stronger types of convergence than in Definition 3.1. Often the convergence (3.4) is uniform in $x \in \mathfrak{M}_{2}$, and there is a characteristic timescale in which decoherence takes place.

In sections 1.2.4 and 1.2.5 we have argued that we should start from a $\mathrm{C}^{*}$-algebra $\mathfrak{A}$ describing the kinematics of the system and then pass to a suitable representation of $\mathfrak{A}$ on a Hilbert space and obtain a von Neumann algebra acting on that Hilbert space. Then, as described in Section 1.3.2 we can pass to a subsystem and study its reduced dynamics and look for effects such as decoherence. It is exactly this procedure that underlies Definition 3.1. However, it is also possible to consider the $\mathrm{C}^{*}$-algebra together with a (reduced) irreversible dynamics and look for a splitting similar to (3.3) on $\mathfrak{A}$. Then one may define an $\mathrm{C}^{*}$-algebra effective observables in a corresponding way. The representation theory of this algebra may then be examined. A concrete example using this procedure was considered in [49]. There, starting from the CCR $\mathrm{C}^{*}$-algebra $\mathfrak{A}\left(\mathbb{R}^{2}\right)$ and a semigroup of completely positive unital maps on it, the $\mathrm{C}^{*}$-algebra of effective observables was found to be $\mathfrak{A}(G)$, where $G$ is the subgroup $\mathbb{Z} \times \mathbb{R}$ of $\mathbb{R}^{2}$. By studying the representation theory of $\mathfrak{A}(G)$ one finds a class of unitarily inequivalent irreducible representations of $\mathfrak{A}(G)$, while $\mathfrak{A}\left(\mathbb{R}^{2}\right)$ has, by the Stone-von Neumann theorem, only one irreducible representation (up to equivalence). This is characteristic for the appearance of superselection rules in the system.

\subsection{Some General Theorems on Decoherence}

\subsubsection{Sufficient Conditions}

Using our results from chapter 2 we can state a number of theorems on decoherence. In particular, we immediately get some sufficient conditions 
for decoherence in case the reduced dynamics is Markovian, i. e. given by a quantum dynamical semigroup.

First we can read the conditions of Theorem 2.3 as sufficient conditions for decoherence. Hence we note the following.

Corollary 3.3 Under the conditions of Theorem 2.3 decoherence takes place.

In particular, the algebra of effective observables $\mathfrak{M}_{1}$ is the image of a normal conditional expectation and (2.9), alternatively Corollary 2.5, gives an explicit characterization of $\mathfrak{M}_{1}$.

Another criterion for the existence of a splitting of the type 3.3 was worked out in Section 2.2.2, Proposition 2.15. We write this result here once again.

Corollary 3.4 Let $\mathfrak{M}$ be a von Neumann algebra and $\left\{T_{t}\right\}_{t \geq 0}$ be an ultraweakly continuous semigroup of contractive operators such that $\omega \circ T_{t} \leq \omega$ for all $t \geq 0$ for a faithful normal state $\omega$ on $\mathfrak{M}$. Then there exist ultraweakly closed $T_{t}$-invariant subspaces $\mathfrak{M}_{\mathrm{s}}$ and $\mathfrak{M}_{\mathrm{r}}$ of $\mathfrak{M}$ such that $\mathfrak{M}=\mathfrak{M}_{\mathrm{s}} \oplus \mathfrak{M}_{\mathrm{r}}$, and

$$
\begin{aligned}
& \mathfrak{M}_{\mathrm{s}}=\left\{x \in \mathfrak{M}: 0 \in{\overline{\left\{T_{t}(x): t \geq 0\right\}}}^{\mathrm{w}^{*}}\right\}, \\
& \mathfrak{M}_{\mathrm{r}}=\overline{\operatorname{lin}\left\{x \in \mathfrak{M}: \exists \alpha \in \mathbb{R} \text { such that } T_{t}(x)=\mathrm{e}^{\mathrm{i} \alpha t} x \forall t \geq 0\right\}}{ }^{\mathrm{w}^{*}} .
\end{aligned}
$$

Moreover, there exists an ultraweakly continuous group $\left\{\alpha_{t}\right\}_{t \in \mathbb{R}} \subseteq$ Aut $\mathfrak{M}_{\mathrm{r}}$ such that $T_{t}\left\lceil\mathfrak{M}_{\mathrm{r}}=\alpha_{t}\right.$ for $t \geq 0$. If $\mathfrak{M}_{\mathrm{s}}$ is a von Neumann subalgebra of $\mathfrak{M}$ or if $\mathfrak{M}_{\mathrm{r}}=\mathfrak{M}_{1}$ (where $\mathfrak{M}_{1}$ is defined by (2.9)) then (3.4) holds for $x \in \mathfrak{M}_{\mathrm{s}}$.

In the situation of this corollary, by the fact that (3.4) holds, we can interpret the splitting $\mathfrak{M}=\mathfrak{M}_{\mathrm{s}} \oplus \mathfrak{M}_{\mathrm{s}}$ in the same way as the splitting (3.3) in Definition 3.1. In general, however, this splitting will be different from the isometric-sweeping splitting of Theorem 2.3, but we always have $\mathfrak{M}_{\mathrm{r}} \subseteq \mathfrak{M}_{1}$. As mentioned in Remark 2.16, if $\mathfrak{M}_{1}=\mathfrak{M}_{\mathrm{r}}$ both splittings agree. This means that, unless $\mathfrak{M}_{1}=\mathfrak{M}_{\mathrm{r}}$, the the reversible algebra $\mathfrak{M}_{\mathrm{r}}$ is not the maximal von Neumann subalgebra on which $\left\{T_{t}\right\}_{t \geq 0}$ is given by a group of automorphisms, as required by Definition 3.1. Indeed, even if $\left\{T_{t}\right\}_{t \geq 0}$ is equal to a group of automorphisms we may have $\mathfrak{M}_{\mathrm{r}}=\mathbb{C} \mathbb{1}$. Still, as illustrated by the example in Section 2.2.3, the above corollary may supply useful information in the examination of decoherence. Thus in view of Definition 3.1, Proposition 2.18 gives a class of quantum dynamical semigroups displaying the decoherence effect. In particular we recall Corollary 2.19 which we can now state as follows. 
Corollary 3.5 Let $\mathfrak{M}$ be a finite-dimensional von Neumann algebra and $\left\{T_{t}\right\}_{t \geq 0}$ a quantum dynamical semigroup (i. e. completely positive and continuous). Then decoherence takes place.

We next return to our discussion of detailed balance in Section 2.3. This leads to a sufficient criterion for decoherence.

Theorem 3.6 Let $\mathfrak{M}$ be a von Neumann algebra, $\left\{T_{t}\right\}_{t \geq 0}$ an ultraweakly continuous semigroup of strongly positive unital maps. Let $\omega$ be a faithful normal state on $\mathfrak{M}$ and suppose that $\left\{T_{t}\right\}_{t \geq 0}$ satisfies detailed balance II with respect to $\omega$. Then $\left\{T_{t}\right\}_{t \geq 0}$ displays decoherence and all conclusions of Theorem 2.3 hold.

Proof. Suppose that $\mathfrak{M}$ acts on $\mathscr{H}$ and, without loss of generality, that $\omega(x)=\left\langle\xi_{0}, x \xi_{0}\right\rangle$ for all $x \in \mathfrak{M}$. Then by Proposition 2.23 it follows that there exists a family of contractions $\left\{\hat{T}_{t}\right\}_{t \geq 0}$ on $\mathscr{H}$ such that $\hat{T}_{t}\left(x \xi_{0}\right)=$ $T_{t}(x) \xi_{0}$ for all $x \in \mathfrak{M}$ and $t \geq 0$. Then $\omega \circ T_{t}=\omega$, and by Theorem 2.29 it follows that $\left[T_{t}, \sigma_{s}^{\omega}\right]=0$ for all $s \in \mathbb{R}$ and $t \geq 0$. Hence Theorem 2.3 applies.

Thus we have shown that detailed balance II implies decoherence. This explains the relation between detailed balance and decoherence, which was discussed in [100], however, without giving a concrete answer. In the situation of Theorem 3.6, the algebra of effective observables $\mathfrak{M}_{1}$ is, of course, given by (2.9), alternatively by Corollary 2.5. However, we now have a third characterization of $\mathfrak{M}_{1}$ as a fixed point space; namely by means of Proposition 2.33 we can write

$$
\mathfrak{M}_{1}=\left\{x \in \mathfrak{M}: T_{t}^{\beta} \circ T_{t}(x)=x \text { for all } t \geq 0\right\} .
$$

We remark that Theorem 2.29 permits us to prove that the condition of Theorem 2.3 are actually equivalent to detailed balance II.

Proposition 3.7 Let $\mathfrak{M}$ be a von Neumann algebra and $\omega$ a faithful normal state on $\mathfrak{M}$. Suppose that $\left\{T_{t}\right\}_{t \geq 0}$ is an ultraweakly continuous semigroup of strongly positive and unital maps. Then $\left\{T_{t}\right\}_{t \geq 0}$ satisfies the assumptions of Theorem 2.3 if and only if $\left\{T_{t}\right\}_{t \geq 0}$ satisfies detailed balance II with respect to $\omega$

Finally we refer to all other results in Section 2.3 on detailed balance, which can be used in the discussion of decoherence because of Theorem 3.6. We shall come back to this point in Chapter 4, where we discuss a concrete application. 


\subsubsection{Structure of the Algebra of Effective Observables}

In the previous section we have seen that the algebra of effective observables $\mathfrak{M}_{1}$ is frequently the image of a normal conditional expectation. We now show that if the time evolution on the effective algebra is trivial then this is always the case. The existence of a normal conditional expectation has a number of consequences for the structure of $\mathfrak{M}_{1}$, some of which we shall derive in the following.

Proposition 3.8 Suppose that $\left\{T_{t}\right\}_{t \geq 0}$ on $\mathfrak{M}$ displays decoherence. Suppose that $T_{t} \uparrow \mathfrak{M}_{1}=\mathrm{id}_{\mathfrak{M}_{1}}$. Then exists a normal conditional expectation $E: \mathfrak{M} \longrightarrow \mathfrak{M}_{1}$ such that $E(\mathfrak{M})=\mathfrak{M}_{1}$, and if $x=x_{1}+x_{2}$ with $x_{i}=\mathfrak{M}_{i}$ for $i=1,2$, then $E(x)=x_{1}$.

Proof. Define $E\left(x_{1}+x_{2}\right)=x_{1}$ for all $x=x_{1}+x_{2} \in \mathfrak{M}$, where $x_{i} \in \mathfrak{M}_{i}$, $i=1,2$. Then $E$ is a linear map such that $E \circ E=E$ and $E(\mathfrak{M})=\mathfrak{M}_{1}$. Since $\mathfrak{M}_{2}$ is *-invariant, we have $E\left(x^{*}\right)=E\left(x_{1}^{*}+x_{2}^{*}\right)=x_{1}^{*}=E(x)^{*}$, hence $E\left(\mathfrak{M}^{\mathrm{sa}}\right) \subseteq \mathfrak{M}^{\mathrm{sa}}$. Let $x \in \mathfrak{M}^{+}$and $x=x_{1}+x_{2}$, then $x_{1}, x_{2} \in \mathfrak{M}^{\mathrm{sa}}$ and $\varphi\left(x_{1}\right), \varphi\left(x_{2}\right)$ is real for any $\varphi \in \mathfrak{M}_{*}^{+}$. Assume that $T_{t} \uparrow \mathfrak{M}_{1}=\mathrm{id}_{\mathfrak{M}_{1}}$ and suppose $0 \neq x_{1}=E(x)$ is not positive. Then there exists $\varphi \in \mathfrak{M}_{*}^{+}$such that $\varphi\left(x_{1}\right)<0$. Now

$$
0 \leq \varphi\left(T_{t}(x)\right)=\varphi\left(T_{t}\left(x_{1}\right)\right)+\varphi\left(T_{t}\left(x_{2}\right)\right),
$$

where $\varphi\left(T_{t}\left(x_{2}\right)\right) \rightarrow 0$ as $t \rightarrow \infty$. This yields a contradiction, hence $x_{1}=$ $E(x) \geq 0$ and $E$ is positive. Now since $E(\mathbb{1})=\mathbb{1}$ it follows that $\|E\| \leq 1$, and from $E \circ E=E$ we get $\|E\| \geq 1$, thus $E$ is a projection of norm 1 and hence a conditional expectation by Tomiyama's theorem. Since ker $E=$ $\left\{x \in \mathfrak{M}: E(x)=x_{1}=0\right\}=\mathfrak{M}_{2}$ is ultraweakly closed, normality follows by a theorem of Tomiyama [128].

A von Neumann algebra $\mathfrak{M}$ is called atomic if the orthomodular lattice $\mathscr{P}(\mathfrak{M})$ is atomic, i. e. if for every nonzero projection $p$ of $\mathfrak{M}$ there exists a nonzero minimal projection $q$ in $\mathfrak{M}$ such that $q \leq p$. The set of minimal projections of $\mathfrak{M}$ is denoted by $\mathscr{P}_{\min }(\mathfrak{M})$.

Lemma 3.9 Let $\mathfrak{M}$ be a von Neumann algebra. Then the following assertions are equivalent:

1. $\mathfrak{M}$ is atomic.

2. There exists an orthogonal family $\left\{p_{i}\right\}_{i \in I} \subseteq \mathscr{P}_{\min }(\mathfrak{M})$ such that $\sum_{i \in I} p_{i}=\mathbb{1}$. 
3. $\mathfrak{M}$ is of type $I$ and there exists an orthogonal family $\left\{p_{i}\right\}_{i \in I} \subseteq$ $\mathscr{P}_{\min }(Z(\mathfrak{M}))$ such that $\sum_{i \in I} p_{i}=\mathbb{1}$.

4. $\mathfrak{M}$ is a direct sum of type I factors.

Proof. $1 . \Rightarrow 2$. Let $\left\{p_{i}\right\}_{i \in I}$ be a maximal family of mutually orthogonal minimal projections in $\mathscr{P}(\mathfrak{M})$ and let $p=\sum_{i \in I} p_{i}$. Then there exists a nonzero minimal projection $q$ in $\mathfrak{M}$ such that $q \leq p^{\perp}$, contradicting maximality.

2. $\Rightarrow 3$. Since a minimal projection is abelian, we find $z_{\mathrm{I}}=\bigvee\{p \in$ $\mathscr{P}(\mathfrak{M}): p$ abelian $\}=\mathbb{1}$, i. e. $\mathfrak{M}$ is of type I. Let $p \in \mathscr{P}_{\min }(\mathfrak{M})$ and let $z_{p}$ be its central support. Then $p \leq z_{p}$. Let $q \in \mathscr{P}(Z(\mathfrak{M}))$ such that $0 \neq q \leq z_{p}$, hence $p q=q p \leq p z_{p}=p$, hence by minimality $q p=p q=p$ or $p \leq q \leq z_{p}$. Thus by definition of $z_{p}$ it follows $q=z_{p}$, hence $z_{p} \in \mathscr{P}_{\min }(Z(\mathfrak{M}))$. Now since in an abelian von Neumann algebra the minimal projections are mutually orthogonal we see that by the assumption they sum up to $\mathbb{1}$.

3. $\Rightarrow$ 4. Put $\mathfrak{M}_{i}=p_{i} \mathfrak{M}=p_{i} \mathfrak{M} p_{i}$ for any $i \in I$, then

$$
\mathfrak{M}=\bigoplus_{i \in I} \mathfrak{M}_{i}
$$

acting on the Hilbert space

$$
\mathscr{H}=\bigoplus_{i \in I} \mathscr{H}_{i}
$$

if $\mathfrak{M}$ acts on $\mathscr{H}$ and $\mathscr{H}_{i}=p_{i} \mathscr{H}$. By minimality of $p_{i}$ we have $p_{i} Z(\mathfrak{M}) p_{i}=$ $p_{i} Z(\mathfrak{M})=\mathbb{C} p_{i}$. Let $p_{i} x \in Z\left(\mathfrak{M}_{i}\right)$, i. e. $0=\left[p_{i} x, p_{i} y\right]=\left[p_{i} x, y\right]$ for all $y \in \mathfrak{M}$, i. e. $p_{i} x \in p_{i} Z(\mathfrak{M})$, hence $\mathfrak{M}_{i}$ is a factor for any $i \in I$. Since $\mathfrak{M}$ is of type I, $p_{i} \mathfrak{M} p_{i}$ is of type I as well.

4. $\Rightarrow 1$. If $\mathfrak{M}=\bigoplus_{i \in I} \mathfrak{M}_{i}$ then any projection $p$ in $\mathfrak{M}$ is of the form $p=\sum_{i \in I}^{\oplus} p_{i}$ with $p_{i} \in \mathscr{P}\left(\mathfrak{M}_{i}\right)$. Since a type I factor is atomic it follows that $\mathfrak{M}$ is atomic.

We remark that (3.5) agrees with the central decomposition provided $\mathfrak{M}$ is countably decomposable (this is necessarily the case if $\mathscr{H}$ is separable), then the index set $I$ is countable.

Lemma 3.10 Let $\mathfrak{M}$ be an atomic von Neumann algebra. Then any $\alpha \in$ Aut $\mathfrak{M}$ fixes $Z(\mathfrak{M})$ pointwise. Moreover, $\alpha$ is inner.

Proof. If $\mathfrak{M}$ is atomic then by Lemma 3.9 there exists an orthogonal family $\left\{p_{i}\right\}_{i \in I} \subseteq \mathscr{P}_{\min }(Z(\mathfrak{M}))$ summing up to $\mathbb{1}$. Since the $\alpha\left(p_{i}\right) \in Z(\mathfrak{M})$ are minimal and mutually orthogonal the claim follows. For the last statement see [23], Proposition 8.9.2. 
In particular, if $\mathfrak{M}$ is atomic and $\left\{\alpha_{t}\right\}_{t \in \mathbb{R}} \subseteq$ Aut $\mathfrak{M}$ is an ultraweakly continuous group of automorphisms we always have $\alpha_{t}(x)=u_{t} x u_{t}^{*}$, where $x \in \mathfrak{M}, t \in \mathbb{R}$, and if $\mathscr{H}$ is separable $\left\{u_{t}\right\}_{t \in \mathbb{R}} \subseteq \mathfrak{M}$ can be taken strongly continuous on $\mathscr{H}$ [84]. Then there exists a self-adjoint operator $H$ on $\mathscr{H}$ such that $u_{t}=\mathrm{e}^{\mathrm{i} t H}$ which is affiliated with $\mathfrak{M}$, i. e. the evolution $\left\{\alpha_{t}\right\}_{t \in \mathbb{R}}$ is Hamiltonian (however, $H$ need not be lower bounded).

A decomposition of $\mathfrak{M}$ as in (3.5) and (3.6) admits an interpretation in terms of superselection rules, the individual components $\left(\mathfrak{M}_{i}, \mathscr{H}_{i}\right)$ are called (superselection) sectors. We notice the following properties of the decomposition (3.6): If $x \in Z(\mathfrak{M})$ then $x=\sum_{i \in I} p_{i} x p_{i}$, and since $p_{i} x \in$ $Z\left(\mathfrak{M}_{i}\right)=p_{i} \mathbb{C}$ we have

$$
x=\sum_{i \in I} \lambda_{i} p_{i}, \quad \lambda_{i} \in \mathbb{C} .
$$

The observables $x=x^{*} \in Z(\mathfrak{M})$ are called superselection observables, any vector in $\mathscr{H}_{i}$ is a common eigenvector of all superselection observables. Another property is the unobservability of phases between different sectors: If $\xi_{i} \in \mathscr{H}_{i}$ and $\xi_{j} \in \mathscr{H}_{j}$ are normalized and $i \neq j$ then $\left\langle\xi_{i}, x \xi_{j}\right\rangle=0$ for any $x \in \mathfrak{M}$, and if $\xi_{\alpha}=\xi_{i}+\mathrm{e}^{\mathrm{i} \alpha} \xi_{j}$ for $\alpha \in \mathbb{R}$ it follows that the expectation value $\left\langle\xi_{\alpha}, x \xi_{\alpha}\right\rangle$ is independent of $\alpha$. Finally, superpositions of vector states between different sectors correspond to mixtures: If $\xi=\alpha_{1} \xi_{i}+\alpha_{x} \xi_{j}$, where $\left|\alpha_{1}\right|^{2}+\left|\alpha_{2}\right|^{2}=1$, then the states $\omega_{\xi}(x)=\langle\xi, x \xi\rangle$ and $\omega(x)=\left|\alpha_{1}\right|^{2} \omega_{\xi_{1}}(x)+\left|\alpha_{2}\right|^{2} \omega_{\xi_{2}}(x)$ agree. Since they are central, superselection observables are classical observables, taking a definite value in each sector. Hence properties associated with superselection observables are classical, and in this way classical properties are obtained. The superposition principle between different sectors is inhibited, moreover, operations from $\mathfrak{M}$ cannot induce transitions between different sectors. From Lemma 3.9 and the definition of the $\mathfrak{M}_{i}$ we see that the sectors $\left(\mathfrak{M}_{i}, \mathscr{H}_{i}\right)$ are minimal in the sense that they cannot be further split up in superselection sectors - they describe systems with a pure quantum character provided $\mathfrak{M}_{i}$ is larger that $\mathbb{C} p_{i}$. We may say that the splitting (3.5) achieves a separation between classical and quantum properties of the system. It is clear that this decomposition is unique. All operations in $\mathfrak{M}$ preserve the superselection sectors, and from Lemma 3.10 it follows that time evolution $\left\{\alpha_{t}\right\}_{t \in \mathbb{R}} \subseteq$ Aut $\mathfrak{M}$ preserves each superselection sector as well.

If $\mathfrak{M}$ is not atomic, we can still obtain a decomposition analogous to (3.5), but which may be a direct integral rather than a direct sum. In general, if $Z(\mathfrak{M}) \neq \mathbb{1} \mathbb{C}$ we have a nontrivial central decomposition of $\mathfrak{M}$ as a direct integral over a measurable field of von Neumann algebras 
$\left\{\mathfrak{M}_{\gamma}\right\}_{\gamma \in \Gamma}$ which are factors,

$$
\begin{aligned}
& \mathfrak{M}=\int_{\Gamma}^{\oplus} \mathfrak{M}(\gamma) \mathrm{d} \mu(\gamma), \\
& \mathscr{H}=\int_{\Gamma}^{\oplus} \mathscr{H}(\gamma) \mathrm{d} \mu(\gamma)
\end{aligned}
$$

Here $\Gamma$ is a standard Borel space and $\mu$ a positive $\sigma$-finite measure (see [30] for the theory of direct integrals). Each von Neumann algebra $\mathfrak{M}(\gamma)$, acting on $\mathscr{H}(\gamma)$, corresponds to a superselection sector in the sense that (3.8) achieves a separation between classical and quantum properties. The interpretation given above in the discrete case is, however, no longer possible since $\mathscr{H}(\gamma)$ is in general not a subspace of $\mathscr{H}$. Indeed, any $\mathfrak{M}$-invariant closed subspace of $\mathscr{H}$ is of the form $\int_{B}^{\oplus} \mathscr{H}(\gamma) \mathrm{d} \mu(\gamma)$, where $B \subseteq \Gamma$ is a Borel set.

The next result is due to Tomiyama [129], see also [4].

Theorem 3.11 A von Neumann algebra $\mathfrak{M}$ acting on $\mathscr{H}$ is atomic if and only if there exists a normal conditional expectation $E$ on $\mathrm{L}(\mathscr{H})$ such that $E(\mathrm{~L}(\mathscr{H}))=\mathfrak{M}$.

From this theorem and Proposition 3.8 we derive the following corollary.

Corollary 3.12 Suppose that an open system with von Neumann algebra $\mathfrak{M}=\mathrm{L}(\mathscr{H})$ displays decoherence, where $\mathscr{H}$ is separable. Suppose that there exists a normal conditional expectation onto $\mathfrak{M}_{1}$. Then the central decomposition of $\mathfrak{M}_{1}$ is given by (3.5) and (3.6), with I countable, i. e. $\mathfrak{M}_{1}$ is a countable direct sum of type I factors, and any automorphism on $\mathfrak{M}$ fixes each direct summand.

This structure of $\mathfrak{M}_{1}$ in the case $\mathfrak{M}=\mathrm{L}(\mathscr{H})$ first been shown in [105] and was further studied in [106], including the dynamics on each direct summand, starting from so-called environment-induced semigroups.

Let $\mathfrak{M}$ be a von Neumann algebra. From a generalization of Theorem 3.11 (cf. [4], IV.2.2.3) it follows that if $\mathfrak{M}_{1}=E(\mathfrak{M})$ is atomic then $\mathfrak{M}$ must be atomic. Hence the effective algebra can only be atomic and hence have the above discrete superselection structure if we start with an atomic algebra. Furthermore, we quote the following results (cf. [4]): If $\mathfrak{M}$ is of type I then $\mathfrak{M}_{1}=E(\mathfrak{M})$ is of type I. If $\mathfrak{M}$ is semifinite then $\mathfrak{M}_{1}$ is semifinite. If $\mathfrak{M}_{1}$ is type III and $E$ is faithful, i.e. $x \geq 0$ and $E(x)=0$ imply $x=0$ then $\mathfrak{M}$ is of type III. The last statement means that if $E$ is faithful a finite system cannot mimic an infinite system by decoherence. 


\subsubsection{Scenarios of Decoherence}

Again consider a quantum system described by a von Neumann algebra $\mathfrak{M}$, acting on a Hilbert space $\mathscr{H}$, and an irreversible dynamics $\left\{T_{t}\right\}_{t \geq 0}$. Suppose that in this system decoherence takes place. By analyzing the structure of the algebra of effective observables $\mathfrak{M}_{1}$ and the automorphism group $\left\{\alpha_{t}\right\}_{t \in \mathbb{R}}$ we can classify some physically relevant situations. In this way we see that, from the general point of view and independent of a concrete model, Definition 3.1 leads to the following scenarios of decoherence. In the following we shall assume throughout that $\mathfrak{M}_{1}$ is the image of a normal conditional expectation.

\section{Environment Induced Superselection Rules}

Suppose that $\mathfrak{M}_{1}$ is a noncommutative algebra and its center $Z\left(\mathfrak{M}_{1}\right)$ is larger than $\mathbb{C} \mathbb{1}$. Then we speak of environment induced superselection rules. Then we have a decomposition of $\mathfrak{M}_{1}$ of the form (3.8) with the interpretation given above. In case $Z\left(\mathfrak{M}_{1}\right)$ is generated by minimal projections and $\mathfrak{M}_{1}$ is countably decomposable, there exists a countable family of minimal central projections adding up to $\mathbb{1}$, and the superselection rules are discrete. We then have a central decomposition as in (3.5) and (3.6). In general, if the evolution $\left\{\alpha_{t}\right\}_{t \in \mathbb{R}}$ fixes the center $Z\left(\mathfrak{M}_{1}\right)$ pointwise then $\left\{\alpha_{t}\right\}_{t \in \mathbb{R}}$ preserves each superselection sector; according to Lemma 3.10 this is the case if $\mathfrak{M}_{1}$ is atomic.

A number of examples showing discrete as well as continuous environment induced superselection rules were given in [46].

\section{Pointer States}

If $\mathfrak{M}_{1}$ is commutative and $\left\{\alpha_{t}\right\}_{t \in \mathbb{R}}$ is trivial we speak of environment induced pointer states. This situation is characteristic for a measuring apparatus (at least in simple situations), where $\mathfrak{M}_{1}$ contains the observables representing the pointer of the apparatus. The commutativity ensures that we obtain a classical probability distribution over the positions whereas the triviality of $\left\{\alpha_{t}\right\}_{t \in \mathbb{R}}$ ensures that the pointer observables are immune to the interaction with the environment, i. e. are not perturbed by it.

In the countably decomposable case, if $\mathfrak{M}_{1}$ is generated by minimal projections $p_{n}, n \in \mathbb{N}$ (i. e. if $\mathfrak{M}_{1}$ is atomic), then $\sum_{n} p_{n}=\mathbb{1}$ and

$$
\mathfrak{M}_{1}=\bigoplus_{n=1}^{\infty} p_{n} \mathfrak{M}, \quad \mathscr{H}=\bigoplus_{n=1}^{\infty} p_{n} \mathscr{H} .
$$


Thus each observable $x \in \mathfrak{M}_{1}$ can be written as $x=\sum_{i=1}^{n} p_{n} x p_{n}$. In this case the evolution $\left\{\alpha_{t}\right\}_{t \in \mathbb{R}}$ is automatically trivial as a consequence of Lemma 3.10.

Models with discrete as well as continuous pointer states (providing a description of a measuring apparatus with continuous readings) were given in [46], see also [47] for another model with continuous pointer states.

\section{Classical System}

Suppose that $\mathfrak{M}_{1}$ is commutative. Then the system can effectively be described in terms of classical probability as explained in Chapter 1. However, in most cases a classical physical system has more structure. For example, the resulting classical probabilistic system and its time evolution, given by $\left\{\alpha_{t}\right\}_{t \in \mathbb{R}}$, need not come from a classical dynamical system, or more precisely, from the Hilbert space representation of a (topological or smooth) classical dynamical system as described in Section 1.2.3. In [46] a general procedure has been laid out to construct a measurable classical dynamical system starting from a commutative von Neumann algebra $\mathfrak{M}_{1}$, an ultraweakly continuous automorphism group $\left\{\alpha_{t}\right\}_{t \in \mathbb{R}}$ and an $\alpha_{t}$-invariant faithful normal weight $\varphi$ on $\mathfrak{M}$. The classical dynamical system is given by a locally compact Hausdorff space $\Omega$ and a $\sigma$-finite $\alpha_{t}$-invariant Borel measure $\mu$ on $\Omega$ such that $\mathfrak{M}_{1}=\mathrm{L}^{\infty}(\Omega, \mu)$, and such that $\left\{\alpha_{t}\right\}_{t \in \mathbb{R}}$ is given by a continuous flow $g_{t}: \mathbb{R} \times M \longrightarrow M$ such that

$$
\alpha_{t}(f)(\omega)=f\left(g_{t}(\omega)\right), \quad f \in \mathfrak{M}_{1}, t \geq 0, \omega \in \Omega .
$$

Thus we see that in this scheme there is room for classical mechanics. Up to now a realistic and physically relevant model in which the appearance of a classical dynamical system has been rigorously established is lacking. See, however, [46] and [94] for mathematical models.

In particular, if we start from $\mathfrak{M}=\mathrm{L}(\mathscr{H})$ then $\mathfrak{M}_{1}=L^{\infty}(\Omega, \mu)$ must be atomic, i. e. the measure $\mu$ must be atomic and hence $\mathfrak{M}_{1}=\ell^{\infty}(\Delta)$, where $\Delta$ is a discrete set, countable if $\mathscr{H}$ is separable. Moreover, the evolution on $\mathfrak{M}_{1}$ is trivial. Thus if we want to reconstruct classical mechanics in our scheme we cannot start from a type I von Neumann algebra.

In the special case where $\mathfrak{M}_{1}=\mathbb{C} \mathbb{1}$, the system displays return to equilibrium in the following sense. There exists a normal state $\omega$ on $\mathfrak{M}$ such that $E(x)=\omega(x) \mathbb{1}$ for all $x \in \mathfrak{M}$. Hence for any normal state $\varphi$ on $\mathfrak{M}$,

$$
\lim _{t \rightarrow \infty} \varphi\left[T_{t}(x)\right]=\lim _{t \rightarrow \infty} \varphi\left[T_{t}\left(E(x)+E^{\perp}(x)\right)\right]=\omega(x)
$$

for any $x \in \mathfrak{M}$. An example of an infinite ergodic bosonic system is given in Section 4.7.2. 


\section{New Quantum Behavior}

If $\mathfrak{M}_{1}$ is again a factor, then together with $\left\{\alpha_{t}\right\}_{t \in \mathbb{R}}$, after decoherence the system effectively still behaves like a system with pure quantum character, which may be, however, smaller than the original system. An example showing this behavior was given in [48]. In particular, the pair $\left(\mathfrak{M}_{1},\left\{\alpha_{t}\right\}_{t \in \mathbb{R}}\right)$ describes a system which is immune to decoherence, thus this scenario might be useful in quantum information theory where it is an essential and difficult problem to have systems available which are immune to decoherence.

\subsubsection{Mesurements}

In this section we shall briefly discuss measurements, mainly to point out the role decoherence plays in the interpretation of measurements in quantum theory. For a more thorough discussion we refer to the literature, see for example [89].

Suppose a quantum system is described by a von Neumann algebra $\mathfrak{M}_{\mathrm{S}}$ and consider an observable $a \in \mathfrak{M}_{\mathrm{S}}$. For simplicity we assume that $a$ has a pure point spectrum $\operatorname{spec} a=\left\{a_{1}, \ldots, a_{n}\right\}$, and that each eigenvalue $a_{i}$ is nondegenerate; we denote the corresponding spectral projector by $q_{i}$ and the corresponding normalized eigenvector in $\mathscr{H}_{\mathrm{S}}$ by $\xi_{i}$. The state of the system is given by the density matrix $\rho$ on $\mathscr{H}_{\mathrm{S}}$. The measuring apparatus is described by the algebra $\mathfrak{M}_{\mathrm{A}}$. Since it is to be handled and its dial to be read by humans it is necessarily a macroscopic and hence an open system, subject to decoherence. Suppose its pointer is described by an observable $x \in \mathfrak{M}_{\mathrm{A}}$. If the apparatus is designed to measure the observable $a$, then we should have $\operatorname{spec} x=\left\{x_{1}, \ldots, x_{n}\right\}$, where the reading $x_{i}$ corresponds to the measurement outcome $a_{i}$. Moreover, there should be a $100 \%$ correlation between the dial reading $x_{i}$ and the state $\xi_{i}$ of the system. If this correlation were not present then the apparatus were not an acceptable instrument to measure $a$. Now since the apparatus is subject to decoherence we expect that there is a superselection rule between different pointer positions, preventing the pointer from being in a superposition of macroscopically distinct states. This superselection rule can be understood on the basis of decoherence as an environment-induced superselection rule. Hence $\mathfrak{M}_{\mathrm{S}, 1}$ has the structure (3.10) with the interpretation of the $p_{i} \mathfrak{M}$ as superselection sectors (here the direct sums are finite). Moreover, the time evolution $\left\{\alpha_{t}\right\}_{t \in \mathbb{R}}$ is trivial on $\mathfrak{M}_{\mathrm{S}, 1}$. Indeed, if the time evolution on the algebra of effective observables would move the pointer of the apparatus its use as a measuring instrument for the observable $a$ would be 
questionable. These two properties, correlation of the pointer positions with the $q_{i}$ and stability of the pointer with respect to time evolution, are the only ones that are necessary for a general discussion of a measurement, the further details of the apparatus do not matter.

The measurement now proceeds in two steps. In the first, the system and the measuring apparatus are brought in interaction This establishes the correlation between the state $\rho$ and the pointer; if $\rho$ is written as

$$
\rho=\sum_{i=1}^{n} \lambda_{i} q_{i}, \quad \lambda_{i} \geq 0, \sum_{i=1}^{n} \lambda_{i}=1,
$$

then by the correlation and the superselection rule governing the pointer the map $\left\{x_{1}, \ldots, x_{n}\right\} \ni x_{i} \mapsto \lambda_{i}$ is the probability distribution of the pointer positions. Notice that since (3.11) is the spectral decomposition of $a$ this probability distribution is the same as the one predicted by the general formalism of Chapter 1. In general, the interaction between the system and the apparatus causes the state $\rho$ of the system to change. According to what we have said in Section 1.3 the effect of the coupling on the system can be described by a (completely) positive normal unital linear map $T$ on $\mathfrak{M}$. Hence the initial state $\rho$ is changed into $T_{*}(\rho)$ as a result of the measurement. If $T$ is of the form

$$
T(x)=\sum_{i=1}^{n} q_{i} x q_{i},
$$

then the measurement is called ideal (a more modern word for it is quantum nondemolition measurement). However, in many situations the measurement is not ideal, for example, during the detection of a particle it is often absorbed in the detector. In the case that the system is macroscopic (and thus classical) it is often argued that the effect of the apparatus on the system can be made arbitrarily small, thus we will have $T \approx \mathbb{1}$ in this case.

The second step of the measurement is the reading of the dial by the observer. Thus he obtains information about the actual reading of the dial, and this gain of information causes him to condition the classical probability distribution $\left\{\lambda_{i}: i=1, \ldots, n\right\}$ according to his observation: If he observes $x_{i}$ (corresponding to $a_{i}$ ) then he will replace the probability distribution with the one concentrated in $x_{i}$. In the case of an ideal measurement, the conclusion is that the system is in state $q_{i}=\left|\xi_{i}\right\rangle\left\langle\xi_{i}\right|$. If only partial information is obtained by the reading, say that the event $E$ has taken place where $E$ is a subset of the sample space $\left\{x_{1}, \ldots, x_{n}\right\}$, then 
the conclusion is that the state is given by

$$
\sum_{i: x_{i} \in E} q_{i} \rho q_{i} / \operatorname{tr}\left(\sum_{i: x_{i} \in E} q_{i} \rho q_{i}\right) .
$$

This is exactly the Lüders-von Neumann reduction formula for the state. 


\section{Chapter 4.}

\section{Irreversible Evolutions on the CCR}

In this chapter we construct dissipative time evolutions on the algebra of the canonical commutation relations (CCR algebra) and representations thereof. To this end we use so-called perturbed convolution semigroups of measures, which are introduced in Section 4.2, where we also discuss their construction. In Section 4.3 we prove two theorems which yield dynamical semigroups on the $\mathrm{C}^{*}$-algebra of canonical commutation relations, given a perturbed convolution semigroup.

Several methods to construct quantum dynamical semigroups on von Neumann algebras have been developed. Uniformly continuous quantum dynamical semigroups have been characterized through their generators [57] by exploiting complete positivity. However, for bosonic systems uniform continuity (or even strong continuity) is a too strong restriction. This is because even the free dynamics on the CCR algebra is given by a group of automorphisms $\left\{\alpha_{t}\right\}_{t \in \mathbb{R}}$ acting on the Weyl operator $W(f)$ as $\alpha_{t}(W(f))=W\left(S_{t}(f)\right)$, where $\left\{S_{t}\right\}_{t \in \mathbb{R}}$ is a group of symplectic linear maps. Since we have $\|W(f)-\mathbb{1}\|=2$ by Lemma A.3 for all $f \in S \backslash\{0\}$, we see that $\left\{\alpha_{t}\right\}_{t \in \mathbb{R}}$ cannot be a strongly continuous group. In order to construct quantum dynamical semigroups on von Neumann algebras in standard form a one-to-one correspondence between dynamical semigroups satisfying a certain symmetry condition and noncommutative Dirichlet forms has been established [58], and a method for constructing certain noncommutative Dirichlet forms has been given [108]. This has been applied to construct an example of a weak* continuous quantum dynamical semigroup on representations of the CCR algebra with respect to quasifree states [43]. However, these methods have not resulted in a rich supply of examples of dynamical semigroups on von Neumann algebras, in particular not on von Neumann algebras corresponding to representations of the CCR. To improve this situation we give in Section 4.4 general con- 
ditions under which the semigroups constructed in Section 4.3 extend to ultraweakly continuous quantum dynamical semigroups on von Neumann algebras associated with representations of the CCR algebra.

In Section 4.5 we explicitly determine the generators of the quantum dynamical semigroups which we have obtained in the previous section. We also give a general criterion in Section 4.6 under which the semigroups have a faithful normal invariant state.

Finally, in Section 4.7 we discuss the detailed balance II condition for quasifree dynamical semigroups on the CCR algebra and study an illustrative example.

\subsection{Quasifree Maps}

In this chapter we will use the algebra of canonical commutation relations (CCR algebra) as introduced in Appendix D, and also the notation that was established there. Throughout, $(\mathrm{S}, \sigma)$ is a real symplectic space with a (possibly degenerate, if not specified otherwise) symplectic form $\sigma$. The CCR $\mathrm{C}^{*}$-algebra corresponding to $(\mathrm{S}, \sigma)$ is denoted by $\mathfrak{A}(\mathrm{S}, \sigma)$ and we use the notation $\mathfrak{A}_{0}(\mathrm{~S}, \sigma)=\operatorname{lin}\{W(f): f \in \mathrm{S}\}$.

We now introduce a very important class of maps on $\mathfrak{A}(\mathrm{S}, \sigma)$, the quasifree maps.

Definition 4.1 A linear unital completely positive (and hence contractive) map $T: \mathfrak{A}(\mathrm{S}, \sigma) \longrightarrow \mathfrak{A}(\mathrm{S}, \sigma)$ is called quasifree if there exists a linear map $S: \mathrm{S} \longrightarrow \mathrm{S}$ and a map $\Gamma: \mathrm{S} \longrightarrow \mathbb{C}$ such that

$$
T(W(f))=\Gamma(f) W(S(f)) \quad \text { for all } f \in \mathrm{S} .
$$

Later on in this chapter we will construct and study dynamical semigroups on $\mathfrak{A}(\mathrm{S}, \sigma)$ of quasifree quasifree maps. Some examples of quasifree maps were given in [67], their systematic study was carried through in $[64,65]$. Given maps $S$ and $\Gamma$ as in Definition 4.1 we can define a map $T$ on $\mathfrak{A}_{0}(\mathrm{~S}, \sigma)$. Then the question arises under which conditions on $S$ and $\Gamma$ this map extends to a completely positive contraction on $\mathfrak{A}(\mathrm{S}, \sigma)$. This question was answered in [64], we quote the result in the following theorem.

Theorem 4.2 Let $S: \mathrm{S} \longrightarrow \mathrm{S}$ be a linear operator and consider the following symplectic form on $\mathrm{S}$,

$$
\sigma_{S}(f, g)=\sigma(f, g)-\sigma(S(f), S(g)), \quad f, g \in \mathrm{S} .
$$

Let $\Gamma: \mathrm{S} \longrightarrow \mathbb{C}$ be a map such that $\Gamma(0)=1$. Then the linear map $T$ 
defined on $\mathfrak{A}_{0}(\mathrm{~S}, \sigma)$ by (4.1) extends to a completely positive unital contraction on the CCR algebra $\mathfrak{A}(\mathrm{S}, \sigma)$ if and only if there exists a state $\omega$ on $\mathfrak{A}\left(\mathrm{S}, \sigma_{S}\right)$ such that $\Gamma=\phi_{\omega}$.

Recall from Appendix D that $\phi_{\omega}$ denotes the generating functional of the state $\omega$.

\subsection{Perturbed Convolution Semigroups}

\subsubsection{Definition}

We can consider the linear space $\mathbf{S}$ as an Abelian group equipped with the discrete topology. We denote its character group, endowed with the Gelfand topology, by $\hat{S}$. A neighborhood base of the Gelfand topology at the identity $e$ of $\hat{\mathrm{S}}$ is given by all sets of the form $\{\chi \in \hat{\mathrm{S}}: \mid \chi(f)-$ $1 \mid<\epsilon$ for all $f \in F\}$ where $F \subseteq \mathrm{S}$ is compact (i. e. finite) and $\epsilon>0$. With this topology $\hat{S}$ is a compact topological group. The algebra of all bounded complex Borel measures on $\hat{\mathrm{S}}$ is denoted by $\mathscr{M}_{\mathrm{b}}(\hat{\mathrm{S}})$ and the set of probability measures by $\mathscr{M}_{1}^{+}(\hat{\mathrm{S}})$. Since $\mathrm{S}$ is discrete every measure in $\mathscr{M}_{\mathrm{b}}(\mathrm{S})$ is absolutely continuous with respect to Haar measure, thus every multiplicative linear functional on the algebra $\mathscr{M}_{\mathrm{b}}(\mathrm{S})$ is of the form $\mu \mapsto \int_{\mathrm{S}} \chi(f) \mathrm{d} \mu(f)$ for some $\chi \in \hat{\mathrm{S}}$ (Theorem 19.20 and Corollary 23.7 in [13]), hence $\hat{S}$ can be identified with the structure space of the Abelian Banach algebra $\mathscr{M}_{\mathrm{b}}(\mathrm{S})$, thus the Gelfand topology on $\hat{\mathrm{S}}$ is the relative $\sigma\left(\mathscr{M}_{\mathrm{b}}(\mathrm{S})^{*}, \mathscr{M}_{\mathrm{b}}(\mathrm{S})\right)$-topology.

For $\mu \in \mathscr{M}_{\mathrm{b}}(\hat{\mathrm{S}})^{+}$recall that the Fourier transform is defined by

$$
\mathrm{A} \cong \mathrm{S} \ni f \mapsto(\mathcal{F} \mu)(f)=\int_{\hat{\mathrm{S}}} \chi(f) \mathrm{d} \mu(\chi),
$$

where $A$ is the character group of $\hat{S}$ which is canonically isomorphic to $S$ by the Pontrjagin-van Kampen theorem. The Fourier transform $\mathcal{F} \mu$ of $\mu$ is a positive-definite functional on S, and by Bochner's theorem any positivedefinite functional on $\mathrm{S}$ is the Fourier transform of a positive measure in $\mathscr{M}_{\mathrm{b}}^{+}(\hat{\mathrm{S}})$. See section 4.3 .2 where we wecall the notion of positivedefiniteness. For any linear map $S: \mathrm{S} \longrightarrow \mathrm{S}$ its dual operator $\hat{S}: \hat{\mathrm{S}} \longrightarrow \hat{\mathrm{S}}$, defined by $(\hat{S} \chi)(f)=\chi(S f), f \in \mathrm{S}, \chi \in \hat{\mathrm{S}}$, is a continuous group homomorphism. Moreover, the map $(\hat{S})_{*}: \mathscr{M}_{1}^{+}(\hat{\mathrm{S}}) \longrightarrow \mathscr{M}_{1}^{+}(\hat{\mathrm{S}})$ is defined by $\left[(\hat{S})_{*} \mu\right](E)=\mu\left(\hat{S}^{-1}(E)\right)$, where $E$ is a Borel set of $\hat{S}$ and $\mu \in \mathscr{M}_{1}^{+}(\hat{S})$. Then $(\hat{S})_{*}$ is an algebra homomorphism. Further concepts and theorems 
from harmonic analysis which will be used in the following can be found in $[13,14]$.

In [49] the concept of a $\left\{\hat{S}_{t}\right\}_{t \geq 0}$-perturbed convolution semigroup was introduced.

Definition 4.3 Let $\left\{S_{t}\right\}_{t \geq 0}$ be a semigroup of linear maps on S. Then $\left\{\mu_{t}\right\}_{t \geq 0} \subseteq \mathscr{M}_{1}^{+}(\hat{\mathrm{S}})$ is called $a\left\{\hat{S}_{t}\right\}_{t \geq 0}$-perturbed convolution semigroup if $\mu_{0}=\delta_{e}$, the Dirac measure concentrated in the unit $e \in \hat{\mathrm{S}}$, and if

$$
\mu_{t} *\left(\hat{S}_{t}\right)_{*} \mu_{s}=\mu_{t+s}, \quad s, t \geq 0 .
$$

It is worth remarking that convolution semigroups satisfying a relation similar to (4.4) were studied independently in the context of generalized Mehler semigroups, see [118, 51, 74].

We shall frequently use the following relation: Let $\mu \in \mathscr{M}_{\mathrm{b}}(\hat{\mathrm{S}})$ and $S: \mathrm{S} \longrightarrow \mathrm{S}$ a linear map. Then

$$
\left(\mathcal{F}(\hat{S})_{*} \mu\right)(f)=\int_{\hat{\mathrm{S}}} \chi(f) \mathrm{d}\left[\hat{S}^{-1} \circ \mu\right](\chi)=\int_{\hat{\mathrm{S}}} \chi(S(f)) \mathrm{d} \mu(\chi)=(\mathcal{F} \mu)(S(f))
$$

for any $f \in \mathrm{S}$.

\subsubsection{Construction of Perturbed Convolution Semigroups}

Suppose for this subsection that the symplectic form $\sigma$ is nondegenerate.

\section{The Dirac Case}

Let $\mathrm{S}^{*}$ be the algebraic dual of $\mathrm{S}$, and for any linear operator $S: \mathrm{S} \longrightarrow \mathrm{S}$ denote by $S^{*}: \mathrm{S}^{*} \longrightarrow \mathrm{S}^{*}$ the corresponding dual operator. For $\psi \in \mathrm{S}^{*}$, $f \mapsto \chi(f)=\mathrm{e}^{\mathrm{i} \psi(f)}$ defines a character in $\hat{\mathrm{S}}$, and any $g \in \mathrm{S}$ defines an element in $\mathrm{S}^{*}$ by $f \mapsto \sigma(f, g)$, thus since $\sigma$ is nondegenerate we have the inclusions $\mathrm{S} \subseteq \mathrm{S}^{*} \subseteq \hat{\mathrm{S}}$.

Proposition 4.4 Let $\psi \in \mathrm{S}^{*}$ and suppose that $t \mapsto \psi\left(S_{t}(f)\right)$ is locally Lebesgue integrable. Define the character $\chi_{t} \in \hat{\mathrm{S}}, \chi_{t}(f)=\mathrm{e}^{\mathrm{i} \psi_{t}(f)}$, where where $\psi_{t}(f)=\int_{0}^{t} \psi\left(S_{r}(f)\right) \mathrm{d} r, f \in \mathrm{S}, t \geq 0$. Then if

$$
\mu_{t}=\delta_{\chi_{t}}, \quad t \geq 0, f \in \mathrm{S},
$$

it follows that $\left\{\mu_{t}\right\}_{t \geq 0}$ is a $\left\{\hat{S}_{t}\right\}_{t \geq 0}$-perturbed convolution semigroup. 
Proof. Let $s, t \geq 0$ and $f \in \mathrm{S}$, then $\psi_{s}\left(S_{t}(f)\right)=\int_{0}^{s} \psi\left(S_{r}\left(S_{t}(f)\right)\right) \mathrm{d} r=$ $\int_{t}^{s} \psi\left(S_{r}(f)\right) \mathrm{d} r$ and we have $\psi_{t}(f)+\psi_{s}\left(S_{t}(f)\right)=\psi_{s+t}(f)$, consequently

$$
\begin{aligned}
\left(\mathcal{F} \mu_{t+s}\right)(f) & =\chi_{s+t}(f)=\chi_{t}(f) \chi_{s}\left(S_{t}(f)\right) \\
& =\left(\mathcal{F} \mu_{t}\right)(f)\left(\mathcal{F} \mu_{s}\right)\left(S_{t}(f)\right) \\
& =\left(\mathcal{F} \mu_{t}\right)(f)\left(\mathcal{F}\left[\left(\hat{S}_{t}\right)_{*} \mu_{s}\right]\right)(f) \\
& =\mathcal{F}\left[\mu_{t} *\left(\hat{S}_{t}\right)_{*} \mu_{s}\right](f)
\end{aligned}
$$

and the assertion is obtained by Fourier inversion.

\section{The Poisson Case}

Proposition 4.5 Let $\nu \in \mathscr{M}_{\mathrm{b}}^{+}(\hat{\mathrm{S}})$ and suppose that $t \mapsto(\mathcal{F} \nu)\left(S_{t}(f)\right)$ is measurable and locally integrable for all $f \in \mathrm{S}$. Then there exists a $\left\{\hat{S}_{t}\right\}_{t \geq 0}$-perturbed convolution semigroup $\left\{\mu_{t}\right\}_{t \geq 0} \subseteq \mathscr{M}_{1}^{+}(\hat{\mathrm{S}})$ such that

$$
\left(\mathcal{F} \mu_{t}\right)(f)=\mathrm{e}^{-t\|\nu\|} \exp \left(\int_{0}^{t}(\mathcal{F} \nu)\left(S_{r}(f)\right) \mathrm{d} r\right), \quad f \in \mathrm{S} .
$$

Proof. The map $f \mapsto(\mathcal{F} \nu)\left(S_{t}(f)\right)$ is positive-definite for each $t \geq 0$, hence the map

$$
f \mapsto \exp \left(\int_{0}^{t}(\mathcal{F} \nu)\left(S_{r}(f)\right) \mathrm{d} r\right)=\sum_{k=0}^{\infty} \frac{1}{k !}\left(\int_{0}^{t}(\mathcal{F} \nu)\left(S_{f}(f)\right) \mathrm{d} r\right)^{k}
$$

is positive-definite as well since the product of positive-definite functions is positive-definite, and by Bochner's theorem there exists a probability measure $\mu_{t} \in \mathscr{M}_{1}^{+}(\hat{\mathrm{S}})$ such that (4.7) holds. Now for all $s, t \geq 0$ and $f \in \mathrm{S}$

$$
\begin{aligned}
\mathcal{F}\left[\mu_{t}\right. & \left.*\left(\hat{S}_{t}\right)_{*} \mu_{s}\right](f)=\left(\mathcal{F} \mu_{t}\right)(f)\left(\mathcal{F} \mu_{s}\right)\left(S_{t}(f)\right) \\
& =\mathrm{e}^{-(t+s)\|\nu\|} \exp \left(\int_{0}^{t}(\mathcal{F} \nu)\left(S_{r}(f)\right) \mathrm{d} r\right) \exp \left(\int_{0}^{s}(\mathcal{F} \nu)\left(S_{t+r}(f)\right) \mathrm{d} r\right) \\
& =\mathrm{e}^{-(t+s)\|\nu\|} \exp \left(\int_{0}^{t+s}(\mathcal{F} \nu)\left(S_{r}(f)\right) \mathrm{d} r\right) \\
& =\left(\mathcal{F} \mu_{t}\right)(f),
\end{aligned}
$$

and we conclude by Fourier inversion that $\left\{\mu_{t}\right\}_{t \geq 0}$ is a $\left\{\hat{S}_{t}\right\}_{t \geq 0}$-perturbed convolution semigroup. 


\section{The Gaussian Case}

A quadratic form is a map $Q: \mathrm{S} \longrightarrow \mathbb{R}$ such that $Q(f+g)+Q(f-g)=$ $2 Q(f)+2 Q(g)$ for all $f, g \in \mathrm{S}$. For any nonnegative quadratic form the map $f \mapsto \mathrm{e}^{-Q(f)}$ is positive-definite (see [3], Theorem 4.3.9. and Schoenberg's theorem, Theorem 3.2.2. of [3]), hence there exists $\mu \in \mathscr{M}_{\mathrm{b}}^{+}(\hat{\mathrm{S}})$ such that $(\mathcal{F} \mu)(f)=\mathrm{e}^{-Q(f)}$ for any $f \in \mathrm{S}$.

Proposition 4.6 Let $Q$ be a nonnegative quadratic form such that $t \mapsto$ $Q\left(S_{t}(f)\right)$ is locally Lebesgue integrable for any $f \in \mathrm{S}$, and define the nonnegative quadratic forms

$$
Q_{t}(f)=\int_{0}^{t} Q\left(S_{r}(f)\right) \mathrm{d} r, \quad t \geq 0, f \in \mathrm{S} .
$$

Then $\left\{\mu_{t}\right\}_{t \geq 0}$ is a $\left\{\hat{S}_{t}\right\}_{t \geq 0}$-perturbed convolution semigroup, where $\mu_{t} \in$ $\mathscr{M}_{1}^{+}(\hat{\mathrm{S}})$ is defined by $\left(\mathcal{F} \mu_{t}\right)(f)=\mathrm{e}^{-Q_{t}(f)}$.

Proof. Since $\mathcal{F}\left[\left(\hat{S}_{t}\right)_{*} \mu_{s}\right](f)=\left(\mathcal{F} \mu_{s}\right)\left(S_{t}(f)\right)=\exp \left(\int_{t}^{t+s} Q\left(S_{r}(f)\right) \mathrm{d} r\right)$ it follows that

$$
\begin{aligned}
\mathcal{F}\left[\mu_{t} *\left(\hat{S}_{t}\right)_{*} \mu_{s}\right](f) & =\left(\mathcal{F} \mu_{t}\right)(f)\left(\mathcal{F} \mu_{s}\right)\left(S_{t}(f)\right) \\
& =\left(\mathcal{F} \mu_{t+s}\right)(f)
\end{aligned}
$$

for any $s, t \geq 0$ and $f \in \mathrm{S}$; the assertion follows by Fourier inversion.

\subsubsection{General Representation of a Perturbed Convolution Semigroup}

Under a regularity condition we will now write the general representation of a $\left\{\hat{S}_{t}\right\}_{t \geq 0}$-perturbed convolution semigroup on $\mathscr{M}_{1}^{+}(\hat{\mathrm{S}})$. The following result has been established in [51] for perturbed convolution semigroups of measures on Hilbert spaces, the proof is literally the same in the present setup.

Proposition 4.7 Let $\left\{\mu_{t}\right\}_{t \geq 0} \subseteq \mathscr{M}_{1}^{+}(\hat{\mathrm{S}})$ be a $\left\{\hat{S}_{t}\right\}_{t \geq 0}$-perturbed convolution semigroup. Assume that $t \mapsto\left(\mathcal{F} \mu_{t}\right)(f)$ is differentiable at $t=0$ for any $f \in \mathrm{S}$ and put

$$
\psi(f)=\left.\frac{\mathrm{d}}{\mathrm{d} t}\left(\mathcal{F} \mu_{t}\right)(f)\right|_{t=0}, \quad f \in \mathrm{S} .
$$

Moreover, assume that $\mathbb{R}^{+} \ni t \mapsto \psi\left(S_{t}(f)\right)$ is locally Lebesgue integrable. Then $-\psi$ is negative-definite and

$$
\left(\mathcal{F} \mu_{t}\right)(f)=\exp \left(\int_{0}^{t} \psi\left(S_{r}(f)\right) \mathrm{d} r\right), \quad f \in \mathrm{S}, t \geq 0 .
$$


Finally, we have $\psi(0)=0$.

Proof. From (4.4) it follows that $\left(\mathcal{F} \mu_{t}\right)(f)\left(\mathcal{F} \mu_{s}\right)\left(S_{t}(f)\right)=\left(\mathcal{F} \mu_{s+t}\right)(f)$ for $s, t \geq 0$. This implies

$$
\frac{1}{s}\left[\left(\mathcal{F} \mu_{t+s}\right)(f)-\left(\mathcal{F} \mu_{t}\right)(f)\right]=\left(\mathcal{F} \mu_{t}\right)(f) \frac{1}{s}\left[\left(\mathcal{F} \mu_{s}\right)\left(S_{t}(f)\right)-1\right],
$$

and using the assumption it follows upon letting $s \downarrow 0$ that $t \mapsto\left(\mathcal{F} \mu_{t}\right)(f)$ is differentiable and

$$
\frac{\mathrm{d}}{\mathrm{d} t}\left(\mathcal{F} \mu_{t}\right)(f)=\left(\mathcal{F} \mu_{t}\right)(f) \psi\left(S_{t}(f)\right), \quad f \in \mathrm{S}, t \geq 0 .
$$

Thus we see that (4.10) satisfies this differential equation and the initial condition $\left(\mathcal{F} \mu_{0}\right)(f)=\left(\mathcal{F} \delta_{e}\right)(f)=1$. Since $\psi$ is the limit of $\frac{1}{t}\left(\mathcal{F} \mu_{t}-1\right)$ it follows that $-\psi$ is a negative-definite function (see the definitions in the next section). Finally, we note that $\psi(0)=0$ since $\left(\mathcal{F} \mu_{t}\right)(0)=1$.

By the previous proposition and Schoenberg's theorem the function $f \mapsto$ $\mathrm{e}^{t \psi(f)}$ is positive-definite, hence there exists a measure $\nu \in \mathscr{M}_{1}^{+}(\hat{\mathrm{S}})$ such that $\mathcal{F} \nu=\mathrm{e}^{\psi}$. It is easily seen that $\nu$ is infinitely divisible: if $n \in \mathbb{N}$ define $\nu_{n} \in \mathscr{M}_{1}^{+}(\hat{\mathrm{S}})$ such that $\mathcal{F} \nu_{n}=\mathrm{e}^{\psi / n}$. Then clearly the $n$-fold convolution power of $\nu$ satisfies $\nu * \cdots * \nu=\nu^{* n}=\nu$.

A continuous function $g: G \times \hat{G} \longrightarrow \mathbb{R}$, where $G$ is a locally compact abelian group with character group $\hat{G}$, is called a Lévy function if the following properties are satisfied:

1. We have $\sup \{|g(x, \chi)|: x \in G, \chi \in K\}<\infty$ for any compact subset $K \subseteq \hat{G}$.

2. The map $g(x, \cdot): \hat{G} \longrightarrow \mathbb{C}$ is a group homomorphism with respect to the additive group of $\mathbb{C}$ for any $x \in G$.

3. For any compact $K \subseteq \hat{G}$ there exists a neighborhood $U_{K}$ of 0 in $G$ such that $\chi(x)=\mathrm{e}^{-\mathrm{i} g(x, \chi)}$ for any $x \in U_{K}$ and $\chi \in K$.

4. We have $\lim _{x \rightarrow 0} g(x, \chi)=0$ uniformly for $\chi$ in compact subsets of $\hat{G}$.

For a locally compact abelian group a Lévy function always exists, see [21, 109]. We will apply this result to the above situation of a symplectic space $\mathrm{S}$ with discrete topology; in the following we take $G=\hat{\mathrm{S}}$ (hence $\hat{G}=\mathrm{S}$ ) and fix a Lévy function $g$. From [70] (see also [3]) the following generalization of the Lévy-Khintchine theorem holds true: There exists a 
group homomorphism $\ell: \mathrm{S} \longrightarrow \mathbb{R}$, a nonnegative quadratic form $q: \mathrm{S} \longrightarrow$ $\mathbb{R}$ and a positive measure $\mu \in \mathscr{M}^{+}(\hat{\mathrm{S}} \backslash\{e\})$ with

$$
\int_{\hat{\mathbf{S}} \backslash\{e\}}(1-\operatorname{Re} \gamma(f)) \mathrm{d} \mu(\gamma)<\infty \text { for all } f \in \mathrm{S},
$$

such that $\psi$ can be written as

$$
-\psi(f)=\psi(0)+\mathrm{i} \ell(f)+q(f)+\int_{\hat{\mathbf{S}} \backslash\{e\}}(1-\gamma(f)+\mathrm{i} g(f, \gamma)) \mathrm{d} \mu(\gamma),
$$

for all $f \in \mathrm{S}$. Recall that $\psi(0)=0$. This yields a general representation of $\psi$ and therefore, by virtue of Proposition 4.7, of general perturbed convolution semigroups. In particular, we see that (up to technicalities) the three cases of Dirac, Gaussian and Poisson perturbed convolution semigroups considered in the previous subsection are exhaustive. There we had considered the special case $\ell(f)=\mathrm{e}^{\psi(f)}$ with $\psi \in \mathrm{S}^{*}$, as well as the case of a bounded $\mu$ with $g=0$.

\subsection{Dynamical Semigroups on $\mathfrak{A}(\mathrm{S}, \sigma)$}

To construct dynamical semigroups on the CCR algebra $\mathfrak{A}(\mathrm{S}, \sigma)$ we will start from a $\left\{\hat{S}_{t}\right\}_{t \geq 0}$-perturbed convolution semigroup $\left\{\mu_{t}\right\}_{t \geq 0}$ on $\hat{\mathrm{S}}$ according to the prescription

$$
\tau_{t}(W(f))=\left(\mathcal{F} \mu_{t}\right)(f) W\left(S_{t}(f)\right), \quad f \in \mathrm{S}, t \geq 0 .
$$

By linear extension this defines a family $\left\{\tau_{t}\right\}_{t \geq 0}$ of maps on $\mathfrak{A}_{0}(\mathrm{~S}, \sigma)$. We have to check that $\tau_{t}$ extends to a completely positive contraction on $\mathfrak{A}(\mathrm{S}, \sigma)$, the semigroup property will then follow from (4.4). First we consider the case when $\left\{S_{t}\right\}_{t \geq 0}$ is a group of symplectic linear maps and later consider the case of a more general $\left\{S_{t}\right\}_{t \geq 0}$.

\subsubsection{Symplectic Groups}

A main result of [49] was the first part of the following theorem which we generalize here by allowing the symplectic form $\sigma$ to be degenerate. In this subsection we assume that $\left\{S_{t}\right\}_{t \in \mathbb{R}}$ is a symplectic group. Some generalizations are provided in the following subsection.

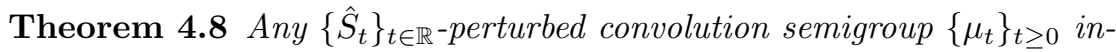
duces a unique semigroup $\left\{\tau_{t}\right\}_{t \geq 0}$ of completely positive contractive and 
unital operators on $\mathfrak{A}(\mathrm{S}, \sigma)$ such that

$$
\tau_{t}(W(f))=\Gamma_{t}(f) W\left(S_{t}(f)\right), \quad f \in \mathrm{S}, t \geq 0,
$$

where $\Gamma_{t}=\mathcal{F} \mu_{t}$ for any $t \geq 0$. Conversely, for any completely positive contractive semigroup $\left\{\tau_{t}\right\}_{t \geq 0}$ on $\mathfrak{A}(\mathrm{S}, \sigma)$ which satisfies (4.12), where $\left\{S_{t}\right\}_{t \in \mathbb{R}}$ is a group of symplectic linear maps, there exists a $\left\{\hat{S}_{t}\right\}_{t \in \mathbb{R}^{-}}$ perturbed convolution semigroup $\left\{\mu_{t}\right\}_{t \geq 0}$ such that $\Gamma_{t}=\mathcal{F} \mu_{t}$ for any $t \geq 0$.

Proof. We will use Theorem 4.2. Since $S_{t}$ is symplectic, $\sigma_{S_{t}}$ is zero and by positive-definiteness of $f \mapsto\left(\mathcal{F} \mu_{t}\right)(f)$ there exists a state $\omega_{t} \in \mathscr{S}(\mathfrak{A}(\mathrm{S}, 0))$ such that $\mathcal{F} \mu_{t}=c_{\omega_{t}}$, hence $\tau_{t}$ extends from $\mathfrak{A}_{0}(\mathrm{~S}, \sigma)$ to a completely positive contraction on $\mathfrak{A}(\mathrm{S}, \sigma)$. It is enough to check the semigroup property on Weyl operators which follows by a calculation using (4.4). Finally, $\tau_{t}(\mathbb{1})=\mathbb{1}$ for all $t \geq 0$ where $\mathbb{1}=W(0)$ is the unit in $\mathfrak{A}(\mathrm{S}, \sigma)$. Conversely, if $\left\{\tau_{t}\right\}_{t \geq 0}$ is a completely positive semigroup of the form (4.12) then there exist states $\omega_{t} \in \mathscr{S}(\mathfrak{A}(\mathrm{S}, 0))$ such that $\Gamma_{t}=c_{\omega_{t}}$, hence this function is positive-definite and by Bochner's theorem there exists $\mu_{t} \in \mathscr{M}_{1}^{+}(\hat{\mathrm{S}})$ such that $\mathcal{F} \mu_{t}=\Gamma_{t}$ for every $t \geq 0$. By the semigroup property,

$$
\begin{aligned}
\Gamma_{s+t}(f) W\left(S_{s+t}(f)\right) & =T_{s+t}(W(f))=T_{s}\left(T_{t}(W(f))\right) \\
& =\Gamma_{t}(f) \Gamma_{s}\left(S_{t}(f)\right) W\left(S_{s+t}(f)\right),
\end{aligned}
$$

for any $s, t \geq 0$ and $f \in \mathrm{S}$. Multiplying by $W\left(S_{s+t}(f)\right)^{*}$ from the left shows that

$$
\Gamma_{t}(f) \Gamma_{s}\left(S_{t}(f)\right)=\Gamma_{s+t}(f),
$$

i. e.

$$
\left(\mathcal{F} \mu_{t}\right)(f) \cdot\left(\mathcal{F} \mu_{s}\right)\left(S_{t}(f)\right)=\left(\mathcal{F} \mu_{s+t}\right)(f),
$$

which is equivalent to (4.4).

\subsubsection{Nonsymplectic Semigroups}

Assume now that the symplectic form $\sigma$ is nondegenerate. Then $\sigma$ induces a dual pairing between $\sigma$ and itself such that the dual $\mathrm{S}^{\prime}$ coincides with $\mathrm{S}$, thus we can consider the corresponding weak topology on $\mathrm{S}$, the $\sigma(\mathrm{S}, \mathrm{S})$ topology, as well as the Mackey topology $\tau(\mathrm{S}, \mathrm{S})$, which is the topology of uniform convergence on compact convex and circled subsets of the dual $\mathrm{S}^{\prime}=\mathrm{S}$. Suppose that $\left\{S_{t}\right\}_{t \geq 0}$ is a semigroup of linear maps on $\mathrm{S}$ which is $\tau(\mathrm{S}, \mathrm{S})$-continuous, i. e., $f \mapsto S_{t}(f)$ and $t \mapsto S_{t}(f)$ are continuous (for fixed $t \geq 0$ and $f \in \mathrm{S}$, respectively) when $\mathrm{S}$ is endowed with 
the $\tau(\mathrm{S}, \mathrm{S})$-topology. Denote by $Z$ the generator of $\left\{S_{t}\right\}_{t \geq 0}$ with respect to this topology, i.e.

$$
\begin{aligned}
\operatorname{dom} Z & =\left\{f \in \mathrm{S}: \lim _{t \downarrow 0} \frac{1}{t}\left(S_{t}(f)-f\right) \text { exists relative to } \tau(\mathrm{S}, \mathrm{S})\right\}, \\
Z f & =\lim _{t \downarrow 0} \frac{1}{t}\left(S_{t}(f)-f\right), \quad f \in \operatorname{dom} Z .
\end{aligned}
$$

Then if $x \in \operatorname{dom} Z$ we have $\frac{\mathrm{d}}{\mathrm{d} t} S_{t}(x)=Z S_{t}(x)=S_{t}(Z x)$ as well as $S_{t}(x)-$ $x=\int_{0}^{t} S_{s}(Z x) \mathrm{d} s$ for any $t \geq 0$, see [85]; the integral is understood as a Riemann integral.

We need to fix some terminology concerning positive- and negativedefinite kernels. Let $\Omega$ be a set. A map $\psi: \Omega \times \Omega \longrightarrow \mathbb{C}$ is called a positive-definite kernel if

$$
\sum_{i, j=1}^{n} \bar{z}_{i} z_{j} \psi\left(x_{i}, x_{j}\right) \geq 0
$$

for all $n \in \mathbb{N}$, and all $x_{1}, \ldots, x_{n} \in \Omega, z_{1}, \ldots, z_{n} \in \mathbb{C}$. If $\Omega$ is an abelian group function $f: \Omega \longrightarrow \mathbb{C}$ is called positive-definite if the kernel $\psi(x, y)=$ $f(x-y), x, y \in \Omega$, is positive-definite. Moreover, $\psi$ is called a negativedefinite kernel if it is hermitian, i. e.

$$
\psi(x, y)=\overline{\psi(y, x)}, \quad x, y \in \Omega,
$$

and if

$$
\sum_{i, j=1}^{n} \bar{z}_{i} z_{j} \psi\left(x_{i}, x_{j}\right) \leq 0
$$

for all $n \in \mathbb{N}$, and all $x_{1}, \ldots, x_{n} \in \Omega, z_{1}, \ldots, z_{n} \in \mathbb{C}$ such that

$$
\sum_{i=1}^{n} z_{i}=0 .
$$

If a kernel is positive-definite, is is hermitian. A function $f: \Omega \longrightarrow \mathbb{C}$ on an abelian group $\Omega$ is called positive- (nenegative)-definite if the kernel $\psi(x, y)=f(x-y), x, y \in \Omega$, is positive- (negative)-definite. The wellknown result we prove next will be needed below, its proof can be found, for example, in [3]. It is essential in the proof of Schoenberg's theorem.

Lemma 4.9 Let $\psi$ be a negative-definite kernel on $\Omega$ and let $x_{0} \in \Omega$. Define the kernel $\varphi: \Omega \times \Omega \longrightarrow \mathbb{C}$ by

$$
\varphi(x, y)=\psi\left(x, x_{0}\right)+\overline{\psi\left(y, x_{0}\right)}-\psi(x, y)-\psi\left(x_{0}, x_{0}\right) .
$$

Then $\varphi$ is positive-definite. 
Proof. Fix $x_{0} \in \Omega$ and let $x_{1}, \ldots, x_{n} \in \Omega$ and $z_{1}, \ldots, z_{n} \in \mathbb{C}$, then define $z_{0}=\sum z_{i}$. Then by the assumption that $\psi$ is negative-definite,

$$
\begin{aligned}
0 \geq & \sum_{i, j=0}^{n} \bar{z}_{i} z_{j} \psi\left(x_{i}, x_{j}\right) \\
= & \sum_{i, j=1}^{n} \bar{z}_{i} z_{j} \psi\left(x_{i}, x_{j}\right)+\sum_{i=1}^{n} \bar{z}_{i} z_{0} \psi\left(x_{i}, x_{0}\right)+\sum_{j=1}^{n} \bar{z}_{0} z_{j} \psi\left(x_{0}, x_{j}\right) \\
& \quad+\left|z_{0}\right|^{2} \psi\left(x_{0}, x_{0}\right) \\
= & \sum_{i, j=1}^{n} \bar{z}_{i} z_{j}\left[\psi\left(x_{i}, x_{j}\right)-\psi\left(x_{i}, x_{0}\right)-\psi\left(x_{0}, x_{j}\right)+\psi\left(x_{0}, x_{0}\right)\right] \\
= & -\sum_{i, j=1}^{n} \bar{z}_{i} z_{j} \varphi\left(x_{i}, x_{j}\right)
\end{aligned}
$$

as was to be shown.

We can now state and prove the following theorem concerning the extension of a certain class of quasifree semigroups to the CCR algebra $\mathfrak{A}(\mathrm{S}, \sigma)$.

Theorem 4.10 Let $\left\{S_{t}\right\}_{t \geq 0}$ be a $\tau(\mathrm{S}, \mathrm{S})$-continuous semigroup on $\mathrm{S}$ with generator $Z$, let $\psi: \mathrm{S} \longrightarrow \mathbb{C}$ be a function such that $\psi(-f)=\overline{\psi(f)}$ for all $f \in \mathrm{S}, \psi(0)=1$, and such that $\mathbb{R}^{+} \ni t \mapsto \psi\left(S_{t}(f)\right)$ is measurable and locally Lebesgue integrable for any $f \in \mathrm{S}$. Define

$$
\Gamma_{t}(f)=\exp \left(\int_{0}^{t} \psi\left(S_{r}(f)\right) \mathrm{d} r\right), \quad f \in \mathrm{S}, t \geq 0,
$$

and define a family $\left\{\tau_{t}\right\}_{t \geq 0}$ of maps on $\mathfrak{A}_{0}(\mathrm{~S}, \sigma)$ by

$$
\tau_{t}(W(f))=\Gamma_{t}(f) W\left(S_{t}(f)\right), \quad f \in \mathrm{S}, t \geq 0
$$

and linear extension. Then $\left\{\tau_{t}\right\}_{t \geq 0}$ extends to a completely positive and unital semigroup on $\mathfrak{A}(\mathrm{S}, \sigma)$ if and only if the kernel $\varphi: \operatorname{dom} Z \times \operatorname{dom} Z \longrightarrow$ $\mathbb{C}$, defined by

$$
\varphi(f, g)=-\frac{\mathrm{i}}{2} \sigma(Z f, g)-\frac{\mathrm{i}}{2} \sigma(f, Z g)-\psi(g-f), \quad f, g \in \operatorname{dom} Z,
$$

is negative-definite. 
Proof. Let $f \in \mathrm{S}$ and $s, t \geq 0$, then from the definitions (4.10) and (4.18) it follows by a simple calculation that

$$
\begin{aligned}
\tau_{s}\left(\tau_{t}(W(f))\right) & =\mathrm{e}^{\int_{0}^{t} \psi\left(S_{r}(f)\right) \mathrm{d} r} \mathrm{e}^{\int_{0}^{s} \psi\left(S_{r}\left(S_{t}(f)\right)\right) \mathrm{d} r} W\left(S_{s+t}(f)\right) \\
& =\exp \left(\int_{0}^{s+t} \psi\left(S_{r}(f)\right) \mathrm{d} r\right) W\left(S_{s+t}(f)\right) \\
& =\tau_{s+t}(W(f)),
\end{aligned}
$$

so $\left\{\tau_{t}\right\}_{t \geq 0}$ is a semigroup on $\mathfrak{A}_{0}(\mathrm{~S}, \sigma)$.

Define the symplectic form $\sigma_{t}(f, g)=\sigma\left(S_{t}(f), S_{t}(g)\right)-\sigma(f, g), f, g \in \mathrm{S}$, as in (4.2). Then by Theorem 4.2, $\tau_{t}$ extends to a completely positive unital contraction on $\mathfrak{A}(\mathrm{S}, \sigma)$ if and only if $f \mapsto \Gamma_{t}(f)$ is a generating functional of a state on $\mathfrak{A}\left(\mathrm{S}, \sigma_{t}\right)$, i. e. by Proposition A.9 if and only if

$$
\sum_{i, j=1}^{n} \bar{z}_{i} z_{j} \mathrm{e}^{\mathrm{i} \sigma_{t}\left(f_{i}, f_{j}\right) / 2} \Gamma_{t}\left(f_{j}-f_{i}\right) \geq 0
$$

for all $n \in \mathbb{N}$, and all $f_{1}, \ldots, f_{n} \in \mathrm{S}, z_{1}, \ldots, z_{n} \in \mathbb{C}$. i. e. if and only if the kernel $\theta_{t}(f, g)=\mathrm{e}^{\mathrm{i} \sigma_{t}(f, g) / 2} \Gamma_{t}(g-f), t \geq 0$, is positive-definite. Suppose that $\theta_{t}$ is positive-definite on $\mathbf{S} \times \mathbf{S}$, and define the kernel

$$
\vartheta_{t}(f, g)=\frac{\mathrm{i}}{2} \sigma_{t}(f, g)+\int_{0}^{t} \psi\left(S_{r}(g-f)\right) \mathrm{d} r
$$

on $\mathrm{S} \times \mathrm{S}$, i. e. $\theta_{t}=\mathrm{e}^{\vartheta_{t}}$ in view of (4.17). If $f, g \in \operatorname{dom} Z$ then both the maps $t \mapsto \vartheta_{t}(f, g)$ and $t \mapsto \theta_{t}(f, g)$ are differentiable. Since $-\frac{1}{t}\left(\theta_{t}(f, g)-1\right)=$ $-\frac{1}{t}\left(\mathrm{e}^{\vartheta_{t}(f, g)}-1\right)$ is negative-definite on $\operatorname{dom} Z \times \operatorname{dom} Z$ it follows that

$$
\begin{aligned}
\varphi(f, g) & =\lim _{t \downarrow 0}-\frac{1}{t}\left(\theta_{t}(f, g)-1\right) \\
& =-\left.\frac{\mathrm{d}}{\mathrm{d} t} \vartheta_{t}(f, g)\right|_{t=0} \\
& =-\frac{\mathrm{i}}{2} \sigma(Z f, g)-\frac{\mathrm{i}}{2} \sigma(f, Z g)-\psi(g-f)
\end{aligned}
$$

is negative-definite, where $f, g \in \operatorname{dom} Z$. Here it was used that for all $f, g \in \mathrm{S}$ we have $\lim _{t \downarrow 0} \vartheta_{t}(f, g)=0$ and that $\left.\frac{\mathrm{d}}{\mathrm{d} t} \sigma\left(S_{t}(f), S_{t}(g)\right)\right|_{t=0}=$ $\sigma(Z f, g)+\sigma(f, Z g)$. This can be proved as follows: For $f, g \in \operatorname{dom} Z$ and $t>0$ we have

$$
\frac{1}{t}\left(\sigma\left(S_{t}(f), S_{t}(g)\right)-\sigma(f, g)\right)=\sigma\left(S_{t}(f), \frac{S_{t}(g)-g}{t}\right)+\sigma\left(\frac{S_{t}(f)-f}{t}, g\right)
$$




$$
\rightarrow \sigma(f, Z g)+\sigma(Z f, g)
$$

as $t \downarrow 0$. The convergence of the first term can be seen as follows. Let $K=\overline{\operatorname{co}}\left\{\lambda S_{t}(f): t \in[0,1], \lambda \in \mathbb{C},|\lambda| \leq 1\right\}$, then $K$ is a $\sigma(\mathrm{S}, \mathrm{S})$ compact convex circled subset of $\mathbf{S}$, hence $p_{K}(f)=\sup \{|\sigma(f, h)|: h \in K\}$ is a Mackey seminorm and we have

$$
\begin{aligned}
\mid \sigma\left(S_{t}(f), \frac{S_{t}(g)-g}{g}\right. & )-\sigma(f, Z g) \mid \\
\leq & \left|\sigma\left(S_{t}(f), \frac{S_{t}(g)-g}{t}-Z g\right)\right|+\left|\sigma\left(S_{t}(f)-f, Z g\right)\right| \\
\leq & p_{K}\left(\frac{S_{t}(g)-g}{t}-Z g\right)+\left|\sigma\left(S_{t}(f)-f, Z g\right)\right| \rightarrow 0
\end{aligned}
$$

as $t \downarrow 0$ by $\tau(\mathrm{S}, \mathrm{S})$-continuity of $\left\{S_{t}\right\}_{t \geq 0}$.

Conversely, if $\varphi$ is negative-definite on $\operatorname{dom} Z \times \operatorname{dom} Z$, so is the kernel

$$
\begin{aligned}
-\vartheta_{t}(f, g):= & \int_{0}^{t} \varphi\left(S_{r}(f), S_{r}(g)\right) \mathrm{d} r \\
= & -\frac{\mathrm{i}}{2} \int_{0}^{t}\left[\sigma\left(Z S_{r}(f), S_{r}(g)\right)+\sigma\left(S_{r}(f), Z S_{r}(g)\right)\right] \mathrm{d} r \\
& \quad-\int_{0}^{t} \psi\left(S_{r}(g-f)\right) \mathrm{d} r \\
= & -\frac{\mathrm{i}}{2} \int_{0}^{t} \frac{\mathrm{d}}{\mathrm{d} r} \sigma\left(S_{r}(f), S_{r}(g)\right) \mathrm{d} r-\int_{0}^{t} \psi\left(S_{r}(g-f)\right) \mathrm{d} r \\
= & -\frac{\mathrm{i}}{2}\left[\sigma\left(S_{t}(f), S_{t}(g)\right)-\sigma(f, g)\right]-\int_{0}^{t} \psi\left(S_{r}(g-f)\right) \mathrm{d} r \\
& =-\frac{\mathrm{i}}{2} \sigma_{t}(f, g)-\int_{0}^{t} \psi\left(S_{r}(g-f)\right) \mathrm{d} r
\end{aligned}
$$

for any $f, g \in \operatorname{dom} Z$ and $t \geq 0$. Then since $-\vartheta_{t}$ is negative-definite it follows by Lemma 4.9 that the kernel

$$
\kappa_{t}(f, g)=-\vartheta_{t}\left(f, f_{0}\right)-\overline{\vartheta_{t}\left(g, f_{0}\right)}+\vartheta_{t}(f, g)+\vartheta_{t}\left(f_{0}, f_{0}\right)
$$

is positive-definite on $\operatorname{dom} Z \times \operatorname{dom} Z$ for an arbitrary $f_{0} \in \operatorname{dom} Z$. Then

$$
\begin{aligned}
\theta_{t}(f, g) & =\mathrm{e}^{\vartheta_{t}(f, g)} \\
& =\mathrm{e}^{\kappa_{t}(f, g)} \mathrm{e}^{\vartheta_{t}\left(f, f_{0}\right)} \mathrm{e}^{\overline{\vartheta_{t}\left(g, f_{0}\right)}} \mathrm{e}^{-\vartheta_{t}\left(f_{0}, f_{0}\right)} \\
& =\mathrm{e}^{\kappa_{t}(f, g)} \kappa^{\prime}(f, g) \mathrm{e}^{-\vartheta_{t}\left(f_{0}, f_{0}\right)}
\end{aligned}
$$


is positive-definite as well as a product of the positive-definite kernels $\mathrm{e}^{\kappa_{t}}$ and $\kappa^{\prime}(f, g)=\mathrm{e}^{\vartheta_{t}\left(f, f_{0}\right)} \mathrm{e}^{\overline{\vartheta_{t}\left(g, f_{0}\right)}}$, and because of $\mathrm{e}^{-\vartheta_{t}\left(f_{0}, f_{0}\right)} \geq 0$. The proof is finished.

Remark 4.11 If $S$ is a complex Hilbert space with inner product $\langle\cdot, \cdot\rangle$ which is considered as a real symplectic space with symplectic form given by $\sigma(f, g)=\operatorname{Im}\langle f, g\rangle$, then the Mackey topology coincides with the norm topology on S. Hence a strongly continuous semigroup is a Mackey continuous semigroup on $\mathrm{S}$.

Remark 4.12 If $\left\{S_{t}\right\}_{t \in \mathbb{R}}$ is a Mackey continuous symplectic group the generator $Z$ satisfies $\sigma(f, Z g)=-\sigma(Z f, g), f, g \in \operatorname{dom} Z$, hence Theorem 4.10 reduces to Theorem 4.8 .

We now consider the case where the function $\psi$ in Theorem 4.10 is a negative quadratic form coming from a bilinear form, i. e. we assume that $\beta: \mathbf{S} \times \mathbf{S} \longrightarrow \mathbb{R}$ is a bilinear form and put $\psi(f)=\beta(f, f)$ for any $f \in \mathbf{S}$. Then $\psi(f+g)+\psi(f-g)=2 \psi(f)+2 \psi(g)$ as well as $\psi(\lambda f)=\lambda^{2} \psi(f)$ for all $f, g \in \mathrm{S}$ and $\lambda \in \mathbb{R}$, moreover, $\psi(f) \leq 0$ for all $f \in \mathrm{S}$. We shall derive a reformulation of the condition (4.19), thus obtaining a criterion for the extendability of the semigroup $\left\{\tau_{t}\right\}_{t \geq 0}$ of (4.18) from $\mathfrak{A}_{0}(\mathrm{~S}, \sigma)$ to a completely positive and unital semigroup on $\mathfrak{A}(\mathrm{S}, \sigma)$.

Proposition 4.13 Let $\psi: \mathrm{S} \longrightarrow \mathbb{R}$ be a negative quadratic form. Then the kernel (4.19) is negative-definite if and only if

$$
\sigma(Z f, g)+\sigma(f, Z g) \leq-2 \psi(f)-2 \psi(g)
$$

for all $f, g \in \operatorname{dom} Z$.

Proof. First observe that $\psi(-f)=\overline{\psi(f)}$. Define the bilinear form $B$ : $\mathrm{S} \times \mathrm{S} \longrightarrow \mathbb{R}$ by $B(f, g)=\psi(f)+\psi(g)-\psi(f-g)$, i. e. the polarization of $\psi$ up to a factor $\frac{1}{2}$. Then the kernel $\varphi$ in (4.19) can be written as

$$
\varphi(f, g)=-\frac{\mathrm{i}}{2} \sigma(Z f, g)-\frac{\mathrm{i}}{2} \sigma(f, Z g)-[\psi(f)+\psi(g)-B(f, g)],
$$

where $f, g \in \operatorname{dom} Z$. Let $f_{1}, \ldots, f_{n} \in \operatorname{dom} Z, z_{1}, \ldots, z_{n} \in \mathbb{C}$ and write $z_{i}=a_{i}+\mathrm{i} b_{i}, i=1, \ldots, n$, and moreover assume that $\sum z_{i}=0$, i.e. $\sum a_{i}=\sum b_{i}=0$. Then

$$
\sum_{i, j=1}^{n} z_{i} \bar{z}_{j} \varphi\left(f_{i}, f_{j}\right)
$$




$$
\begin{aligned}
= & \sum_{i, j=1}^{n}\left(a_{i} a_{j}+b_{i} b_{j}-\mathrm{i} a_{i} b_{j}+\mathrm{i} b_{i} a_{j}\right) . \\
& \cdot\left(-\frac{\mathrm{i}}{2} \sigma\left(Z f_{i}, f_{j}\right)-\frac{\mathrm{i}}{2} \sigma\left(f_{i}, Z f_{j}\right)-\psi\left(f_{i}\right)-\psi\left(f_{j}\right)+B\left(f_{i}, f_{j}\right)\right) .
\end{aligned}
$$

Let us consider the terms on the right hand side of this expression individually. We have

$$
\sum_{i, j=1}^{n} a_{i} a_{j} \sigma\left(Z f_{i}, f_{j}\right)=\sigma\left(Z \sum_{i=1}^{n} a_{i} f_{i}, \sum_{j=1}^{n} a_{j} f_{j}\right)=\sigma(Z f, f),
$$

where we define $f=\sum a_{i} f_{i}, g=\sum b_{i} f_{i}$. We have by antisymmetry

$$
\sigma(Z f, f)+\sigma(f, Z f)=0,
$$

which implies

$$
\sum_{i, j=1}^{n}\left(a_{i} a_{j}+b_{i} b_{j}\right)\left(\sigma\left(Z f_{i}, f_{j}\right)+\sigma\left(f_{i}, Z f_{j}\right)\right)=0 .
$$

Moreover,

$$
\sum_{i, j=1}^{n} a_{i} a_{j} \psi\left(f_{j}\right)=\left(\sum_{i=1}^{n} a_{i}\right) \sum_{j=1}^{n} a_{j} \psi\left(f_{j}\right)=0
$$

similarly,

$$
\sum_{i, j=1}^{n} a_{i} b_{j} \psi\left(f_{j}\right)=\sum_{i, j=1}^{n} b_{i} b_{j} \psi\left(f_{j}\right)=0 .
$$

Finally,

$$
\sum_{i, j=1}^{n}\left(a_{i} b_{j}-b_{i} a_{j}\right) B\left(f_{i}, f_{j}\right)=0
$$

by symmetry of $B$. When these and similar expressions are taken together (4.21) can be written as

$$
\begin{aligned}
\sum_{i, j=1}^{n} z_{i} \bar{z}_{j} \varphi\left(f_{i}, f_{j}\right)= & \frac{\mathrm{i}}{2} \sum_{i, j=1}^{n} a_{i} b_{j}\left(\mathrm{i} \sigma\left(f_{i}, Z f_{j}\right)+\mathrm{i} \sigma\left(Z f_{i}, f_{j}\right)\right) \\
& -\frac{\mathrm{i}}{2} \sum_{i, j=1}^{n} b_{i} a_{j}\left(\mathrm{i} \sigma\left(f_{i}, Z f_{j}\right)+\mathrm{i} \sigma\left(Z f_{i}, f_{j}\right)\right)
\end{aligned}
$$




$$
\begin{aligned}
& +\sum_{i, j=1}^{n}\left(a_{j} a_{j}+b_{i} b_{j}\right) B\left(f_{i}, f_{j}\right) \\
=- & \frac{1}{2}(\sigma(f, Z g)+\sigma(Z f, g))+\frac{1}{2}(\sigma(g, Z f)+\sigma(Z g, f)) \\
& \quad+B(f, f)+B(g, g) \\
= & \sigma(g, Z f)+\sigma(Z g, f)+2 \psi(f)+2 \psi(g) \\
\leq & 0
\end{aligned}
$$

in view of the assumption (4.20). Hence condition (4.19) of Theorem 4.10 holds. Conversely, suppose that $\varphi$ is negative-definite. Choose $n=2$ and define $a_{1}=-a_{2}=1, b_{1}=-b_{2}=1$ as well as $f=f_{1}, g=g_{1} \in \operatorname{dom} Z$, and $f_{2}=0, g_{2}=0$. Then the previous calculation shows that (4.20) holds.

We now study a simple example to which the preceding proposition applies.

Example 4.14 Let $\mathrm{S}=\mathbb{C}^{2} \cong \mathbb{R}^{2}$ with symplectic form $\sigma(f, g)=\operatorname{Im} \bar{f} g=$ $(2 \mathrm{i})^{-1}(\bar{f} g-f \bar{g}), f, g \in \mathrm{S}$. Then the Weyl relations (A.5) read

$$
W(f) W(g)=\mathrm{e}^{-(\bar{f} g-f \bar{g}) / 4} W(f+g) .
$$

Define the quadratic form

$$
\psi(f)=-\theta|f|^{2}, \quad \theta>0, f \in S
$$

and the semigroup

$$
S_{t}(f)=\mathrm{e}^{-\gamma t / 2} f, \quad \gamma>0, t \geq 0,
$$

hence $Z f=-\frac{1}{2} \gamma f$. Then

$$
\Gamma_{t}(f)=\exp \left(-\theta \int_{0}^{t} \mathrm{e}^{\gamma r}|f|^{2} \mathrm{~d} r\right)=\exp \left(\frac{\theta}{\gamma}\left(\mathrm{e}^{-\gamma t}-1\right)|f|^{2}\right) .
$$

Then

$$
\begin{aligned}
\sigma(Z f, g)+\sigma(f, Z g) & =-\gamma \sigma(f, g)=-\gamma \operatorname{Im} \bar{f} g \\
& \leq \gamma|f g| \leq \frac{1}{2} \gamma\left(|f|^{2}+|g|^{2}\right) \\
& =\frac{\gamma}{2 \theta}(-\psi(f)-\psi(g))
\end{aligned}
$$

Hence we see that condition (4.20) is satisfied if $\gamma \leq 4 \theta$. 


\subsection{Extension to Representations of the CCR Algebra}

Let us fix a symplectic space $(\mathrm{S}, \sigma)$ with a nondegenerate symplectic form $\sigma$ and consider the CCR algebra $\mathfrak{A}(\mathrm{S})=\mathfrak{A}(\mathrm{S}, \sigma)$. We will give sufficient conditions for a semigroup $\left\{\tau_{t}\right\}_{t \geq 0}$ of the form (4.12) with a symplectic group $\left\{S_{t}\right\}_{t \in \mathbb{R}}$ to be $\omega$-covariant-see Definition B.6-for a state $\omega \in$ $\mathscr{S}(\mathfrak{A}(\mathrm{S}))$, i. e. we wil construct a quantum dynamical semigroup $\left\{T_{t}\right\}_{t \geq 0}$ on the von Neumann algebra $\mathfrak{M}=\pi_{\omega}(\mathfrak{A}(\mathrm{S}))^{\prime \prime}$ satisfying

$$
T_{t}\left(\pi_{\omega}(W(f))\right)=\pi_{\omega}\left(\tau_{t}(W(f))\right), \quad f \in \mathrm{S}, t \geq 0,
$$

i. e. (B.9). Hence our goal is to "extend" a quasifree dynamical semigroup on the CCR algebra $\mathfrak{A}(\mathrm{S})$ to the von Neumann algebra corresponding to a representation of $\mathfrak{A}(\mathrm{S})$. To achieve this "extension" we shall use the tools developed in Appendix B as well as results from Appendices C and D.

\subsubsection{The Extension Theorem}

Consider a group of symplectic linear maps $\left\{S_{t}\right\}_{t \in \mathbb{R}}$. By Proposition A.10 it induces a group of $*_{\text {-automorphisms }}\left\{\alpha_{t}\right\}_{t \in \mathbb{R}}$ on $\mathfrak{A}(\mathrm{S})$ such that

$$
\alpha_{t}(W(f))=W\left(S_{t}(f)\right), \quad f \in \mathrm{S}, t \in \mathbb{R} .
$$

Let $\omega \in \mathscr{S}(\mathfrak{A}(\mathrm{S}))$ be a state on $\mathfrak{A}(\mathrm{S})$, let $\left(\pi_{\omega}, \mathscr{H}_{\omega}, \xi_{\omega}\right)$ be the corresponding GNS-triplet, and denote the corresponding von Neumann algebra by

$$
\mathfrak{M}=\pi_{\omega}(\mathfrak{A}(\mathrm{S}))^{\prime \prime} .
$$

As an abbreviation write

$$
W_{\omega}(f)=\pi_{\omega}(W(f)), \quad f \in \mathrm{S},
$$

and recall that the $W_{\omega}(f)$ also satisfy (A.5) (see Theorem A.5). Moreover,

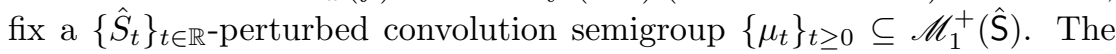
conditions E 1-E 6 listed below will be shown to be sufficient for the semigroup $\left\{\tau_{t}\right\}_{t \geq 0}$ on $\mathfrak{A}(\mathrm{S})$ given by (4.12) in Theorem 4.8 to be $\omega$-covariant. Moreover, in this case the corresponding semigroup $\left\{T_{t}\right\}_{t \geq 0}$ given by (B.9) on $\mathfrak{M}$ consists of completely positive unital operators. The conditions read as follows:

E 1 The map $f \mapsto\left(\mathcal{F} \mu_{t}\right)(f)$ is $\sigma(\mathrm{S}, \mathrm{S})$ continuous. 
E 2 The map $t \mapsto \mu_{t} \in \mathscr{M}_{\mathrm{b}}(\hat{\mathrm{S}})$ is vaguely continuous.

E 3 The map $f \mapsto W_{\omega}(f)$ is measurable when $\mathrm{S}$ is endowed with $\sigma(\mathscr{U} \mathrm{S})$ and $\mathfrak{M}$ with the Borel $\sigma$-algebra of the weak operator topology.

E 4 The group $\left\{\alpha_{t}\right\}_{t \in \mathbb{R}}$ is $\omega$-covariant, i. e. there exists a group of *-automorphisms $\left\{\beta_{t}\right\}_{t \in \mathbb{R}} \subseteq$ Aut $\mathfrak{M}$ such that

$$
\pi_{\omega}\left(\alpha_{t}(W(f))\right)=\beta_{t}\left(W_{\omega}(f)\right), \quad f \in \mathrm{S}, t \in \mathbb{R} .
$$

E 5 The map $t \mapsto \pi_{\omega}\left(\alpha_{t}(W(f))\right)=W_{\omega}\left(S_{t}(f)\right)$ is continuous in the weak operator topology (i.e. $\left\{\alpha_{t}\right\}_{t \in \mathbb{R}}$ is $\omega$-continuous).

E 6 The Hilbert space $\mathscr{H}_{\omega}$ is separable.

Proposition 4.15 Suppose that $\omega,\left\{\mu_{t}\right\}_{t \geq 0}$, and $\left\{S_{t}\right\}_{t \in \mathbb{R}}$ satisfy conditions E1-E6. Then the semigroup $\left\{\tau_{t}\right\}_{t \geq 0}$ constructed in Theorem 4.8 with the property (4.12) is $\omega$-covariant. The corresponding semigroup $\left\{T_{t}\right\}_{t \geq 0}$ on $\mathfrak{M}$ uniquely defined by (B.9) is ultraweakly contiunous, completely positive and unital. Moreover, $\left\{T_{t}\right\}_{t \geq 0}$ is the unique ultraweakly continuous semigroup on $\mathfrak{M}$ satisfying

$$
T_{t}\left(W_{\omega}(f)\right)=\left(\mathcal{F} \mu_{t}\right)(f) W_{\omega}\left(S_{t}(f)\right), \quad f \in \mathrm{S}, t \geq 0 .
$$

Proof. Endow $\mathrm{S}$ with the $\sigma(\mathrm{S}, \mathrm{S})$ topology. Since for each $t \geq 0$ the map $f \mapsto\left(\mathcal{F} \mu_{t}\right)$ is positive-definite and continuous when restricted to finitedimensional subspaces of $S$ there exists by Theorem D.1 a positive normalized cylindrical measure $\nu_{t}$ on $\mathscr{U}_{\mathrm{S}}$ such that

$$
\left(\mathcal{F} \mu_{t}\right)(f)=\int_{\mathrm{S}} \mathrm{e}^{\mathrm{i} \sigma(f, g)} \mathrm{d} \nu_{t}(g), \quad f \in \mathrm{S}, t \geq 0 .
$$

By Proposition C.6 the $\sigma(\mathrm{S}, \mathrm{S})$ topology on $\mathrm{S}$ is nuclear, hence it coincides with the Sazonov topology. Thus by Theorem D.2 and assumption E 1 each $\nu_{t}$ is $\sigma$-additive and extends to a measure on $\sigma\left(\mathscr{U}_{\mathrm{S}}\right)$. Thus we can define on $\mathfrak{M}$ the operators $V_{t}$ by an integral with respect to $\nu_{t}$ over a noncylindrical function:

$$
V_{t}(x)=\int_{\mathrm{S}} W_{\omega}(g) x W_{\omega}(g)^{*} \mathrm{~d} \nu_{t}(g), \quad x \in \mathfrak{M}, t \geq 0 .
$$

This is well-defined since the integrand is weakly measurable by E 3 . The $V_{t}$ are completely positive, unital and normal. Using E 6 normality can be shown in the same way as the weak ${ }^{*}$-weak ${ }^{*}$ continuity of $R_{\lambda}$ in the proof of Lemma B.2. By (A.5) it is easy to see that we have

$$
V_{t}\left(W_{\omega}(f)\right)=\left(\mathcal{F} \mu_{t}\right)(f) W_{\omega}(f), \quad f \in \mathrm{S}, t \geq 0 .
$$


Using E 4 we define on $\mathfrak{M}$ the operators $T_{t}$ by

$$
T_{t}(x)=\left(\beta_{t} \circ V_{t}\right)(x), \quad x \in \mathfrak{M}, t \geq 0 .
$$

Clearly, the $T_{t}$ are normal, completely positive, and unital. Using (4.4) and the properties of Fourier transform one can show that $\left\{T_{t}\right\}_{t \geq 0}$ satisfies the semigroup property $T_{s} \circ T_{t}=T_{s+t}$ for $s, t \geq 0$, and $T_{0}=\mathbb{1}$, by checking it on Weyl operators. By construction the $T_{t}$ satisfy (4.23), and in particular $T_{t}\left(W_{\omega}(f)\right)=\pi_{\omega}\left(\tau_{t}(W(f))\right)$. By a density argument this relation implies (B.9). It remains to prove the ultraweak continuity of $\left\{T_{t}\right\}_{t \geq 0}$. Define $\mathfrak{B}=\operatorname{lin}\left\{W_{\omega}(f): f \in \mathrm{S}\right\}$. By (A.5) it follows that $\mathfrak{B}$ is a ultraweakly dense *-subalgebra of $\mathfrak{M}$ and $\mathbb{1} \in \mathfrak{B}$. Since $t \mapsto\left(\mathcal{F} \mu_{t}\right)(f)$ is continuous by $\mathrm{E} 2$ and $t \mapsto W_{\omega}\left(S_{t}(f)\right)$ is ultraweakly continuous by $\mathrm{E} 5$ it follows that $t \mapsto T_{t}\left(W_{\omega}(f)\right)=\left(\mathcal{F} \mu_{t}\right)(f) W_{\omega}\left(S_{t}(f)\right)$ is ultraweakly continuous for each $f \in \mathrm{S}$. This property extends to finite linear combinations of Weyl operators, hence the map $t \mapsto T_{t}(x), x \in \mathfrak{B}$, is ultraweakly continuous. Using E 6 the conclusion now follows from Corollary B.5.

We make some remarks about the validity of conditions E 1-E 6 .

1. A natural embedding $\mathrm{S} \hookrightarrow \hat{\mathrm{S}}$ is given by the identification of $g \in \mathrm{S}$ with the character $\chi_{g} \in \hat{\mathrm{S}}$ defined by $\chi_{g}(f)=\mathrm{e}^{\mathrm{i} \sigma(f, g)}$. If $\operatorname{supp} \mu_{t} \subseteq \mathrm{S}$ for all $t \geq 0$ condition $\mathrm{E} 1$ becomes redundant because we have by definition of the Fourier transform

$$
\left(\mathcal{F} \mu_{t}\right)(f)=\int_{\mathrm{S}} \mathrm{e}^{\mathrm{i} \sigma(f, g)} \mathrm{d} \mu_{t}(g), \quad f \in \mathrm{S}, t \geq 0,
$$

which replaces (4.24) in the proof of the proposition.

2. Assume that $\mathrm{S}$ is a complex separable Hilbert space with inner product $\langle\cdot, \cdot\rangle$, considered as a symplectic space by defining $\sigma(f, g)=\operatorname{Im}\langle f, g\rangle$, $f, g \in \mathrm{S}$. Then it can be shown that $\sigma\left(\mathscr{U}_{\mathrm{S}}\right)$ is the Borel $\sigma$-algebra of the norm topology of $\mathrm{S}$, therefore $\mathrm{E} 3$ can be rephrased by requiring norm-toweak operator measurability.

3. If there exist unitaries $u_{t} \in \mathrm{L}\left(\mathscr{H}_{\omega}\right)$ such that $\pi_{\omega}\left(\alpha_{t}(x)\right)=u_{t} \pi_{\omega}(x) u_{t}^{*}$ for all $t \geq 0$ and $x \in \mathfrak{A}(\mathrm{S})$ condition $\mathrm{E} 4$ is satisfied. See [52] for a number of sufficient conditions for the existence of such $u_{t}$. In particular, this is the case if $\omega \circ \alpha_{t}=\omega$ for all $t \in \mathbb{R}$. Moreover, if there exists a cyclic and separating vector for $\mathfrak{M}$ in $\mathscr{H}_{\omega}$, it follows from Tomita-Takesaki theory (see Corollary 2.5.32. of [6]) that $\left\{\alpha_{t}\right\}_{t \in \mathbb{R}} \subseteq$ Aut $\mathfrak{A}$ is unitarily implementable if and only if it is $\omega$-covariant.

The following example shows that the conditions E 1-E 6 are satisfied in situations of physical interest. 
Example 4.16 We consider the temperature representation of a free Bose gas. Let $\mathrm{S}=L^{2}\left(\mathbb{R}^{d}\right)$ and assume that $H$ is the free Hamiltonian, i. e. the unique self-adjoint extension of $-\nabla^{2}$ to $\mathrm{S}$. Let $\beta>0$ and $\mu \in \mathbb{R}$ such that $H \geq(\mu+c) \mathbb{1}$ for some $c>0$. If we put $z=\mathrm{e}^{\beta \mu}$ it can be shown that

$$
\omega(W(f))=\exp \left(-\frac{1}{4}\left\langle f,\left(\mathbb{1}+z \mathrm{e}^{-\beta H}\right)\left(\mathbb{1}-z \mathrm{e}^{-\beta H}\right)^{-1} f\right\rangle\right), \quad f \in \mathrm{S},
$$

defines a gauge invariant quasifree state on $\mathfrak{A}(\mathrm{S})$. This state is the thermal equilibrium state of a free Bose gas in the high temperature-low density (noncondensed) regime. Let $\left\{S_{t}\right\}_{t \in \mathbb{R}}$ be given by $S_{t}(f)=\mathrm{e}^{\mathrm{i} t H} f$, then clearly $S_{t}$ is symplectic. For details and proofs of the above facts we refer to [7], see also Section 1.2.5. By Proposition 5.2.29. of [7] the following properties hold: The map $f \mapsto W_{\omega}(f)$ is strongly continuous when $\mathbf{S}$ is endowed with the norm topology, hence E 3 is satisfied. There exists a strongly continuous unitary group $\left\{u_{t}\right\}_{t \in \mathbb{R}}$ on $\mathscr{H}_{\omega}$, defined by

$$
u_{t} x \xi_{\omega}=\alpha_{t}(x) \xi_{\omega}, \quad x \in \mathfrak{A}(\mathrm{S})
$$

such that $\pi_{\omega}\left(\alpha_{t}(x)\right)=u_{t} \pi_{\omega}(x) u_{t}^{*}=\beta_{t}\left(\pi_{\omega}(x)\right), x \in \mathfrak{A}(\mathrm{S})$, thus $\mathrm{E} 4$ and $\mathrm{E} 5$ are satisfied. Finally, let $\mathrm{S}_{0}$ be a countable dense set in $\mathrm{S}$. Then it is easy to see that the set

$$
\left\{\sum_{j=1}^{n}\left(a_{j}+\mathrm{i} b_{j}\right) W_{\omega}\left(f_{j}\right) \xi_{\omega}: a_{j}, b_{j} \in \mathbb{Q}, f_{j} \in \mathrm{S}_{0}, n \in \mathbb{N}\right\} \subseteq \mathscr{H}_{\omega}
$$

is countable and dense in $\mathscr{H}_{\omega}$, hence $\mathrm{E} 6$ is satisfied. Summarizing, we have shown that for a thermal equilibrium state of a noncondensed free Bose gas all conditions depending on the representation, i. e. E 3-E 6, are satisfied.

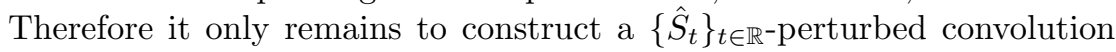
semigroup satisfying conditions E1 and E 2, see Section 4.2.2 and [49].

\subsubsection{A Generalization of the Extension Theorem}

We now show that if $\mathbf{S}$ can be densely embedded in a larger symplectic space $\overline{\mathrm{S}}$, then, for certain states $\omega$, the condition $\operatorname{supp} \mu_{t} \subseteq \overline{\mathrm{S}}$ for $t \geq 0$ implies the conclusion of Proposition 4.15. Let $\mathcal{T}(\mathrm{S}, \sigma)$ be the set of all topologies $\tau$ on the symplectic space $(\mathrm{S}, \sigma)$ such that the maps $\mathrm{S} \times \mathrm{S} \ni$ $(f, g) \mapsto f+g$ and $\mathbf{S} \times \mathbf{S} \ni(f, g) \mapsto \sigma(f, g)$ are (jointly) $\tau$-continuous and such that $\mathbb{R} \ni t \mapsto t f$ is $\tau$-continuous. For every $\tau \in \mathcal{T}(\mathrm{S}, \sigma)$ define

$$
F_{\tau}(\mathrm{S}, \sigma)=\left\{\omega \in \mathscr{S}(\mathfrak{A}(\mathrm{S}, \sigma)): f \mapsto \phi_{\omega}(f) \text { is } \tau \text {-continuous }\right\}
$$


remark that a characteristic function $\phi_{\omega}$ is $\tau$-continuous on $\mathbf{S}$ if and only if it is $\tau$-continuous at 0 , see [80]. Then it can be shown [80] that $F_{\tau}(\mathrm{S}, \sigma)$ is a folium and that every state in $F_{\tau}(\mathrm{S}, \sigma)$ is regular.

Let $(\overline{\mathrm{S}}, \bar{\sigma})$ be a symplectic space with a nondegenerate symplectic form $\bar{\sigma}$ and equipped with some topology $\tau \in \mathcal{T}(\overline{\mathrm{S}}, \bar{\sigma})$ such that the symplectic space $(\mathrm{S}, \sigma)$ is $\tau$-dense in $\overline{\mathbf{S}}$ and $\bar{\sigma} \mid \mathbf{S}=\sigma$. Note that because $\bar{\sigma}$ is nondegenerate and $\mathrm{S} \subseteq \overline{\mathrm{S}}$ is dense we have the chain of inclusions $\mathrm{S} \subseteq \overline{\mathrm{S}} \subseteq$ $\hat{\overline{\mathrm{S}}} \subseteq \hat{\mathrm{S}}$. It has been shown in [80] that $\omega \in F_{\tau}(\mathrm{S}, \sigma)$ extends to a unique state $\bar{\omega} \in F_{\tau}(\overline{\mathrm{S}}, \bar{\sigma})$ and that the corresponding GNS-representations have the property

$$
\pi_{\bar{\omega}}\left\lceil\mathfrak{A}(\mathrm{S})=\pi_{\omega}, \quad \mathscr{H}_{\bar{\omega}}=\mathscr{H}_{\omega}, \quad \text { and } \quad \xi_{\bar{\omega}}=\xi_{\omega} .\right.
$$

Wew now establish the generalization of Proposition 4.15.

Proposition 4.17 Let $\overline{\mathrm{S}}$ and $\omega$ be as above, and let $\left\{\mu_{t}\right\}_{t \geq 0} \subseteq \mathscr{M}_{1}^{+}(\hat{\mathrm{S}})$

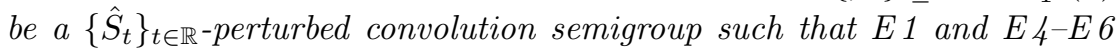
are satisfied. Moreover, assume that $\overline{\mathrm{S}} \ni f \mapsto W_{\bar{\omega}}(f)$ is $\sigma(\hat{\mathrm{S}}, \mathrm{S})-$ weak operator measurable. If $\operatorname{supp} \mu_{t} \subseteq \overline{\mathrm{S}}$ for all $t \geq 0$ then the conclusion of Proposition 4.15 holds true.

Proof. Using the canonical inclusion $\overline{\mathrm{S}} \subseteq \hat{\mathrm{S}}$ and the assumption supp $\mu_{t} \subseteq$ $\overline{\mathrm{S}}$ we can write as in remark 1 .

$$
\left(\mathcal{F} \mu_{t}\right)(f)=\int_{\hat{\mathrm{S}}} \chi(f) \mathrm{d} \mu_{t}(\chi)=\int_{\overline{\mathrm{S}}} \mathrm{e}^{\mathrm{i} \bar{\sigma}(f, g)} \mathrm{d} \mu_{t}(g), \quad f \in \mathrm{S} t \geq 0 .
$$

Defining

$$
V_{t}(x)=\int_{\overline{\mathrm{S}}} W_{\bar{\omega}}(g) x W_{\bar{\omega}}(g)^{*} \mathrm{~d} \mu_{t}(g), \quad x \in \mathfrak{M}, t \geq 0,
$$

we see that $V_{t}\left(W_{\omega}(f)\right)=\left(\mathcal{F} \mu_{t}\right)(f) W_{\omega}(f)$ for all $f \in \mathrm{S}, t \geq 0$, and $V_{t}(\mathfrak{M}) \subseteq$ $\mathfrak{M}$, where $\mathfrak{M}=\pi_{\omega}(\mathfrak{A}(\mathrm{S}))^{\prime \prime}$. We now proceed as in the proof of Proposition 4.15 .

The preceding proposition can be applied to the following situations: Let the symplectic space $S$ in the problem at hand be the Schwartz space $\mathscr{S}\left(\mathbb{R}^{d}\right)$ or the test function space $\mathscr{D}\left(\mathbb{R}^{d}\right)$, and take $\overline{\mathrm{S}}=L^{2}\left(\mathbb{R}^{d}\right)$ with symplectic form $\bar{\sigma}(f, g)=\operatorname{Im}\langle f, g\rangle$, the imaginary part of the $L^{2}$ inner product, and let $\sigma$ be the restriction of $\bar{\sigma}$ to $\mathrm{S}$. Then the norm topology on $L^{2}\left(\mathbb{R}^{d}\right)$ is in $\mathcal{T}(\overline{\mathrm{S}}, \bar{\sigma})$. 


\subsection{Generators}

In Section 4.2.2 we constructed Dirac, Gaussian, and Poisson $\left\{\hat{S}_{t}\right\}_{t \in \mathbb{R}}$-perturbed convolution semigroups which, by Theorem 4.8, induce semigroups on the CCR algebra. In this section we will give, for each case, conditions for them to be $\omega$-covariant given a state state $\omega$ on the CCR algebra $\mathfrak{A}(\mathrm{S})$ and calculate the generators of their extensions to the von Neumann algebra associated with the representation $\pi_{\omega}$ of the CCR algebra.

Throughout this section let us fix the following assumptions and notation. Let $(\mathrm{S}, \sigma)$ be a symplectic space with a nondegenerate symplectic form $\sigma$ and let $\left\{S_{t}\right\}_{t \in \mathbb{R}}$ be a group of symplectic linear maps. To assure the $\sigma(\mathrm{S}, \mathrm{S})$ continuity of functions the form

$$
\mathrm{S} \ni f \mapsto \int_{0}^{t} F\left(S_{r}(f)\right) \mathrm{d} r
$$

where $F: \mathrm{S} \longrightarrow \mathbb{R}$ is a $\sigma(\mathrm{S}, \mathrm{S})$ continuous function, we assume that the $\sigma(\mathrm{S}, \mathrm{S})$ topology satisfies the first axiom of countability in order to characterize continuity by sequential convergence and make the dominated convergence theorem applicable. This assumption is satisfied, for example, if $\mathrm{S}$ is a complex separable pre-Hilbert space (e. g. Schwartz space $\mathscr{S}\left(\mathbb{R}^{d}\right)$ ) considered as a symplectic space in the canonical way. Moreover, we shall assume throughout the rest of this chapter that $\left\{S_{t}\right\}_{t \in \mathbb{R}}$ is weakly measurable, i. e. that the maps

$$
\mathbb{R} \ni t \mapsto \sigma\left(S_{t}(f), g\right)
$$

are measurable for all $f, g \in \mathrm{S}$.

We start with a general lemma. The set of all infinitely differentiable functions on the interval ]0, $\infty$ [ having compact support is denoted by $\mathrm{C}_{\mathrm{c}}^{\infty}(] 0, \infty[)$.

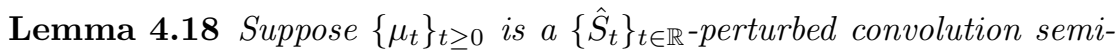
group satisfying E 1-E 6 for $\bar{a}$ state $\omega$ and let $\left\{T_{t}\right\}_{t \geq 0}$ be the semigroup given by Proposition 4.15. Let $\delta$ be the generator of the automorphism group $\left\{\beta_{t}\right\}_{t \in \mathbb{R}} \subseteq$ Aut $\mathfrak{M}$ defined in (4.22) and let $Z$ be the generator of $\left\{T_{t}\right\}_{t \geq 0}$. Define

$$
\mathfrak{B}=\operatorname{lin}\left\{W_{\omega}(f): f \in \mathbf{S}\right\}
$$

and

$$
C=\left\{\int_{0}^{\infty} \zeta(t) T_{t}(x) \mathrm{d} t: x \in \mathfrak{B}, \zeta \in \mathrm{C}_{\mathrm{c}}^{\infty}(] 0, \infty[)\right\}
$$


Then $C$ is ultraweakly dense in $\mathfrak{M}, C \subseteq \operatorname{dom} Z, C \subseteq \operatorname{dom} \delta$, and $C$ is a core for both $Z$ and $\delta$.

Proof. Let $C^{\circ}=\left\{\varphi \in \mathfrak{M}_{*}:\langle x, \varphi\rangle=0 \forall x \in C\right\}$ be the polar of $C$. Let $\varphi \in$ $C^{\circ}$, then $0=\int_{0}^{\infty} \zeta(t)\left\langle T_{t}(x), \varphi\right\rangle \mathrm{d} t$ for all $x \in \mathfrak{B}$ and $\zeta \in \mathrm{C}_{\mathrm{c}}^{\infty}(] 0, \infty[)$, thus the continuous function $t \mapsto\left\langle T_{t}(x), \varphi\right\rangle$ vanishes on $] 0, \infty[$, hence $\langle x, \varphi\rangle=0$ for all $x \in \mathfrak{B}$, hence $\varphi=0$ and we have $C^{\circ}=\{0\}$. Now the bipolar theorem implies $\mathfrak{M}=C^{\circ \circ}=\bar{C}$. Let $x_{\zeta}=\int_{0}^{\infty} \zeta(t) T_{t}(x) \mathrm{d} t \in C$, then

$$
\frac{1}{s}\left(T_{s}\left(x_{\zeta}\right)-x_{\zeta}\right)=\frac{1}{s} \int_{0}^{\infty} \zeta(t)\left[T_{s}\left(T_{t}(x)\right)-T_{t}(x)\right] \mathrm{d} t,
$$

and since $T_{t}(x) \in \operatorname{dom} Z$ if $t>0$ we obtain by the dominated convergence theorem upon letting $s \downarrow 0$ that

$$
Z x_{\zeta}=\int_{0}^{\infty} \zeta(t) \frac{\mathrm{d}}{\mathrm{d} t} T_{t}(x) \mathrm{d} t=\int_{0}^{\infty} \zeta(t) Z\left(T_{t}(x)\right) \mathrm{d} t, \quad x \in \mathfrak{B},
$$

thus $C \subseteq \operatorname{dom} Z$. Clearly $T_{t}(C) \subseteq C$, therefore $C$ is a core for $Z$. Similarly, if $x_{\zeta}=\int_{0}^{\infty} \zeta(t) T_{t}\left(W_{\omega}(f)\right) \mathrm{d} t=\int_{0}^{\infty} \zeta(t) \Gamma_{t}(f) \beta_{t}\left(W_{\omega}(f)\right) \mathrm{d} t$ for $f \in \mathrm{S}$ and $\zeta \in \mathrm{C}_{\mathrm{c}}^{\infty}(] 0, \infty[)$, then as before

$$
\frac{1}{s}\left(\beta_{s}\left(x_{\zeta}\right)-x_{\zeta}\right)=\frac{1}{s} \int_{0}^{\infty} \zeta(t) \Gamma_{t}(f)\left[\beta_{s}\left(\beta_{t}\left(W_{\omega}(f)\right)\right)-\beta_{t}\left(W_{\omega}(f)\right)\right] \mathrm{d} t .
$$

Since $\beta_{t}\left(W_{\omega}(f)\right) \in \operatorname{dom} \delta$ if $t>0$ we get by letting $s \downarrow 0$

$$
\begin{aligned}
\delta\left(x_{\zeta}\right) & =\int_{0}^{\infty} \zeta(t) \Gamma_{t}(f) \delta\left(\beta_{t}\left(W_{\omega}(f)\right)\right) \mathrm{d} t \\
& =\int_{0}^{\infty} \zeta(t) \delta\left(T_{t}\left(W_{\omega}(f)\right)\right) \mathrm{d} t .
\end{aligned}
$$

We conclude that $C \subseteq \operatorname{dom} \delta$, and since $\beta_{t}\left(W_{\omega}(f)\right)=W_{\omega}\left(S_{t}(f)\right)$ we also see that $\beta_{t}(C) \subseteq C$, thus $C$ is a core for $\delta$.

\subsubsection{The Gaussian Case}

Let $Q: \mathrm{S} \longrightarrow \mathbb{R}$ be a nonnegative quadratic form. We assume throughout that $\mathbb{R}^{+} \ni t \mapsto Q\left(S_{t}(f)\right)$ is measurable and locally integrable. Define the quadratic forms $Q_{t}$ by (4.8) and there exists a unique measure $\mu_{t} \in \mathscr{M}_{1}^{+}(\hat{\mathrm{S}})$ such that

$$
\left(\mathcal{F} \mu_{t}\right)(f)=\Gamma_{t}(f)=\mathrm{e}^{-Q_{t}(f)}, \quad f \in \mathrm{S}, t \geq 0 .
$$




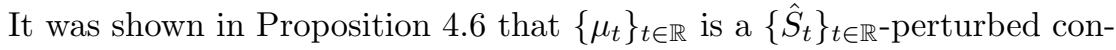
volution semigroup. We say that $\left\{\mu_{t}\right\}_{t \geq 0}$ and as well as the corresponding semigroup $\left\{\tau_{t}\right\}_{t>0}$ on $\mathfrak{A}(\mathrm{S})$ constructed in Theorem 4.8 are of Gaussian type.

If $f \mapsto Q(f)$ is $\tau_{\mathrm{n}}(\mathrm{S}, \sigma(\mathrm{S}, \mathrm{S}))=\sigma(\mathrm{S}, \mathrm{S})$ continuous then $f \mapsto Q_{t}(f)$ is $\sigma(\mathrm{S}, \mathrm{S})$ continuous as well because $S_{t}$ is symplectic, thus in this case E 1 is satisfied, and E 2 is clearly satisfied as well.

If $t \mapsto W_{\omega}(t f)$ is strongly continuous for all $f \in \mathrm{S}$ the state $\omega$ is regular and we can introduce the field operators $\Phi_{\omega}(f), f \in \mathrm{S}$, of the Weyl system $\left\{W_{\omega}(f): f \in \mathrm{S}\right\}$ as described in Appendix A. Recall that $\xi_{\omega} \in \operatorname{dom} \Phi_{\omega}(f)$ for all $f \in \mathrm{S}$ if and only if $t \mapsto \omega(W(t f))$ is twice differentiable for all $f \in \mathrm{S}$. (In particular, this condition is satisfied for all quasifree states.)

Lemma 4.19 Assume that $\xi_{\omega} \in \operatorname{dom} \Phi_{\omega}(f)$ for all $f \in \mathrm{S}$. Moreover, assume that conditions $E 1-E 6$ are satisfied for a semigroup $\left\{\tau_{t}\right\}_{t \geq 0}$ on $\mathfrak{A}(\mathrm{S})$ of the form (4.12). Then it follows that

$$
D:=\operatorname{lin}\left\{W_{\omega}(g) \xi_{\omega}: g \in \mathrm{S}\right\} \subseteq \operatorname{dom} \Phi_{\omega}(f) \quad \text { for all } f \in \mathrm{S}
$$

and

$$
\begin{aligned}
{\left[\Phi_{\omega}\left(g_{1}\right),\left[\Phi_{\omega}\left(g_{2}\right), \int_{0}^{\infty} \zeta(t) T_{t}\left(W_{\omega}(f)\right) \mathrm{d} t\right]\right] \xi } & \\
= & \int_{0}^{\infty} \sigma\left(g_{1}, S_{t}(f)\right) \sigma\left(g_{2}, S_{t}(f)\right) \zeta(t) T_{t}\left(W_{\omega}(f)\right) \xi \mathrm{d} t
\end{aligned}
$$

for all $g_{1}, g_{2}, f \in \mathrm{S}, \xi \in D$, and all $\int_{0}^{\infty} \zeta(t) T_{t}\left(W_{\omega}(f)\right) \mathrm{d} t \in C$, where $\zeta \in \mathrm{C}_{\mathrm{c}}^{\infty}(] 0, \infty[)$. The double commutator in (4.33) is well-defined.

Proof. By Proposition A.14 it follows that for all $f, g \in \mathrm{S}$ and for all $\xi \in D$ we have the commutation relation

$$
\left[\Phi_{\omega}(f), W_{\omega}(g)\right] \xi=-\sigma(f, g) W_{\omega}(g) \xi .
$$

Moreover, $T_{t}\left(W_{\omega}(f)\right) \xi \in \operatorname{dom} \Phi_{\omega}(g)$, and by $\mathrm{E} 4$ the map $t \mapsto T_{t}\left(W_{\omega}(f)\right)$ is ultraweakly and hence weak operator continuous, hence the integrals

$$
\int_{0}^{\infty} \zeta(t) T_{t}\left(W_{\omega}(f)\right) \xi \mathrm{d} t, \quad \int_{0}^{\infty} \zeta(t) \Phi_{\omega}(g) T_{t}\left(W_{\omega}(f)\right) \xi \mathrm{d} t
$$

exist in $\mathscr{H}_{\omega}$ as weak* integrals. If $\eta \in \operatorname{dom} \Phi_{\omega}(g)$ then

$$
\left\langle\Phi_{\omega}(g) \eta, \int_{0}^{\infty} \zeta(t) T_{t}\left(W_{\omega}(f)\right) \xi \mathrm{d} t\right\rangle=\int_{0}^{\infty} \zeta(t)\left\langle\Phi_{\omega}(g) \eta, T_{t}\left(W_{\omega}(f)\right) \xi\right\rangle \mathrm{d} t
$$




$$
=\left\langle\eta, \int_{0}^{\infty} \zeta(t) \Phi_{\omega}(g) T_{t}\left(W_{\omega}(f)\right) \xi \mathrm{d} t\right\rangle
$$

and we see that $\int_{0}^{\infty} \zeta(t) T_{t}\left(W_{\omega}(f)\right) \xi \mathrm{d} t \in \operatorname{dom} \Phi_{\omega}^{*}(g)=\operatorname{dom} \Phi_{\omega}(g)$. In the same way we can show that $\int_{0}^{\infty} \zeta(t) \Phi_{\omega}\left(g_{1}\right) T_{t}\left(W_{\omega}(f)\right) \xi \mathrm{d} t \in \operatorname{dom} \Phi_{\omega}\left(g_{2}\right)$, i. e. $\int_{0}^{\infty} \zeta(t) T_{t}\left(W_{\omega}(t)\right) \xi \mathrm{d} t \in \operatorname{dom}\left(\Phi_{\omega}\left(g_{2}\right) \Phi_{\omega}\left(g_{1}\right)\right)$ for all $f, g_{1}, g_{2} \in \mathrm{S}$ and $\xi \in D$. By a similar procedure we obtain also $\int_{0}^{\infty} T_{t}\left(W_{\omega}(f)\right) \Phi_{\omega}\left(g_{1}\right) \xi \mathrm{d} t \in$ $\operatorname{dom} \Phi_{\omega}\left(g_{2}\right)$ for all $f, g_{1}, g_{2} \in \mathrm{S}$ and $\xi \in D$. Using these results we see upon expanding the double commutator on the left hand side of (4.33) that it is well-defined, and by applying (4.34) to the left hand side of (4.33) we obtain the desired result.

In what follows we will write the bounded operator on $\mathscr{H}_{\omega}$ defined by

$$
D \ni \xi \mapsto\left[\Phi_{\omega}(f), x\right] \xi=\Phi_{\omega}(f) x \xi-x \Phi_{\omega}(f) \xi,
$$

where $x \in C$, formally as a commutator $\left[\Phi_{\omega}(f), x\right]$.

Proposition 4.20 Let $\left\{\mu_{t}\right\}_{t \geq 0}$ be a Gaussian $\left\{\hat{S}_{t}\right\}_{t \in \mathbb{R} \text {-perturbed convo- }}$ lution semigroup defined by (4.31) with a quadratic form $Q: \mathrm{S} \longrightarrow \mathbb{R}^{+}$ such that $f \mapsto Q(f)$ is $\tau_{\mathrm{n}}(\mathrm{S}, \sigma(\mathrm{S}, \mathrm{S}))$ continuous. Assume that $\omega$ is regular and $\xi_{\omega} \in \operatorname{dom} \Phi_{\omega}(f)$ for all $f \in \mathrm{S}$, and that E 3-E 6 are satisfied. Then the corresponding semigroup $\left\{\tau_{t}\right\}_{t>0}$ on $\mathfrak{A}(\mathrm{S})$ given by Theorem 4.8 is $\omega$-covariant and the generator $Z$ of the quantum dynamical semigroup $\left\{T_{t}\right\}_{t \geq 0}$ on $\mathfrak{M}$ induced by the covariance condition (B.9) is given by

$$
Z x=\delta(x)-\sum_{k=1}^{\infty} \lambda_{k}\left[\Phi_{\omega}\left(f_{k}\right),\left[\Phi_{\omega}\left(g_{k}\right), x\right]\right], \quad x \in C,
$$

where $\left\{f_{k}\right\}_{k \in \mathbb{N}},\left\{g_{k}\right\}_{k \in \mathbb{N}}$ are $\sigma(\mathrm{S}, \mathrm{S})$ equicontinuous sequences in $\mathrm{S}$ and the sequence $\left\{\lambda_{k}\right\}_{k \in \mathbb{N}} \subseteq \mathbb{R}$ satisfies

$$
\sum_{k=1}^{\infty}\left|\lambda_{k}\right|<\infty .
$$

Proof. First we notice that E $1-\mathrm{E} 6$ are satisfied and the semigroup $\left\{T_{t}\right\}_{t \geq 0}$ given by (4.23) exists and is ultraweakly continuous. By polarization the bilinear form $B: \mathrm{S} \times \mathrm{S} \longrightarrow \mathbb{R}$ corresponding to $Q$ via $Q(f)=B(f, f)$ is jointly $\sigma(\mathrm{S}, \mathrm{S})$ continuous. The nuclear kernel theorem [25] and the nuclearity of $\sigma(\mathrm{S}, \mathrm{S})$, see Proposition C.6, imply the existence of equicontinuous sequences $\left\{f_{k}\right\}_{k \in \mathbb{N}},\left\{g_{k}\right\}_{k \in \mathbb{N}} \subseteq \mathrm{S}^{\prime}=\mathrm{S}$ (in particular, the sequences 
are contained in a compact set by the Alaoglu-Bourbaki theorem) and an absolutely summable sequence $\left\{\lambda_{k}\right\}_{k \in \mathbb{N}} \subseteq \mathbb{R}$ such that

$$
B(f, g)=\sum_{k=1}^{\infty} \lambda_{k} \sigma\left(f, f_{k}\right) \sigma\left(g, g_{k}\right), \quad f, g \in \mathrm{S} .
$$

Now observe that $t \mapsto \Gamma_{t}(f)$ is differentiable almost everywhere by the Lebesgue fundamental theorem of calculus. Let

$$
x_{\zeta}=\int_{0}^{\infty} \zeta(t) T_{t}\left(W_{\omega}(f)\right) \mathrm{d} t \in C
$$

where $f \in \mathrm{S}$, then from (4.29) and (4.30) we have

$$
\begin{aligned}
Z x_{\zeta} & =\int_{0}^{\infty} \zeta(t) \frac{\mathrm{d}}{\mathrm{d} t}\left[\Gamma_{t}(f) \beta_{t}\left(W_{\omega}(f)\right)\right] \mathrm{d} t \\
& =\int_{0}^{\infty} \zeta(t) \Gamma_{t}(f)\left[\delta\left(\beta_{t}\left(W_{\omega}(f)\right)\right)-Q\left(S_{t}(f)\right) W_{\omega}\left(S_{t}(f)\right)\right] \mathrm{d} t \\
& =\delta\left(x_{\zeta}\right)-\sum_{k=1}^{\infty} \lambda_{k} \int_{0}^{\infty} \zeta(t) \sigma\left(S_{t}(f), f_{k}\right) \sigma\left(S_{t}(f), g_{k}\right) T_{t}\left(W_{\omega}(f)\right) \mathrm{d} t \\
& =\delta\left(x_{\zeta}\right)-\sum_{k=1}^{\infty} \lambda_{k}\left[\Phi_{\omega}\left(f_{k}\right),\left[\Phi_{\omega}\left(g_{k}\right), x_{\zeta}\right]\right],
\end{aligned}
$$

where we have used Lemma 4.19, the expansion of the bilinear form (4.37), and the dominated convergence theorem for series together with the weak measurability of $\left\{S_{t}\right\}_{t \in \mathbb{R}}$ and local integrability of $t \mapsto Q_{t}(f)$. We conclude that (4.36) holds for all $x \in C$.

The result of the preceding proposition can be sharpened if $\mathrm{S}$ carries a complex structure, i.e. there exists a complex pre-Hilbert space $\mathrm{H}$ with inner product $\langle\cdot, \cdot\rangle$ such that the real vector space $\mathrm{S}$ equals $\mathrm{H}$ and $\sigma(f, g)=$ $\operatorname{Im}\langle f, g\rangle$ for all $f, g \in \mathrm{S}$. Let $J: \mathrm{S} \longrightarrow \mathrm{S}$ be given by $J f=\mathrm{i} f, f \in \mathrm{S}$. The completion of $\mathbf{H}$ is denoted by $\tilde{H}$. The nuclear topology $\tau_{\mathrm{n}}(\mathrm{H},\|\cdot\|)$ is induced by the seminorms $q(x)=\|\rho x\|$, where $\rho$ is a Hilbert-Schmidt operator on $\tilde{\mathrm{H}}$. In the following corollary we use the characterization of a nuclear operator given in Theorem C.1.

Corollary 4.21 Let all assumptions be as in Proposition 4.20 except that we now assume that $\mathrm{S}$ carries a complex structure and that $Q(f)=\langle f, A f\rangle$ where $A$ is a nuclear positive $\mathbb{C}$-linear operator on $\mathrm{H}$. Then $\left\{\tau_{t}\right\}_{t \geq 0}$ is $\omega$ covariant and the generator $Z$ of the corresponding quantum dynamical 
semigroup $\left\{T_{t}\right\}_{t \geq 0}$ is given by

$$
Z x=\delta(x)-\sum_{k=1}^{\infty} \lambda_{k}\left[\Phi_{\omega}\left(e_{k}\right),\left[\Phi_{\omega}\left(e_{k}\right), x\right]\right], \quad x \in C,
$$

where $\left\{e_{k}\right\}_{k \in \mathbb{N}} \subseteq \mathrm{H}$ is a norm bounded sequence and $\left\{\lambda_{k}\right\}_{k \in \mathbb{N}} \subseteq \mathbb{R}^{+}$is a sequence such that

$$
\sum_{k=1}^{\infty} \lambda_{k}<\infty
$$

Proof. Since $A$ is nuclear and positive it is easy to show that the map $f \mapsto Q(f)=\langle f, A f\rangle$ is $\tau_{\mathrm{n}}(\mathrm{S}, \sigma(\mathrm{S}, \mathrm{S}))$ continuous, thus E 1-E 6 are satisfied. From Proposition C.2 it follows that $A$ has an extension $\tilde{A}$ to $\tilde{\mathrm{H}}$ such that $\tilde{A}$ is nuclear and positive. Now there exists an orthonormal basis $\left\{e_{k}^{\prime}\right\}_{k \in \mathbb{N}} \subseteq \tilde{\mathrm{H}}$ and a summable sequence $\left\{\lambda_{k}^{\prime}\right\}_{k \in \mathbb{N}}$ with $\lambda_{k}^{\prime} \geq 0$ such that

$$
\tilde{A} f=\sum_{k=1}^{\infty} \lambda_{k}^{\prime}\left\langle e_{k}^{\prime}, f\right\rangle e_{k}^{\prime}
$$

Since $A$ is nuclear on $\mathbf{H}$ it is compact (Proposition C.2). Now let $f \in \tilde{\mathbf{H}}$, then there exists a sequence $\left\{f_{n}\right\}_{n \in \mathbb{N}}$ such that $f_{n} \rightarrow f$ in norm, and by compactness (after passing to a subsequence) we can assume that $A f_{n} \rightarrow$ $y \in \mathrm{H}$. But then $\tilde{A} f=y$ and we conclude $\tilde{A}(\tilde{\mathrm{H}}) \subseteq \mathrm{H}$, in particular $\tilde{A} e_{k}^{\prime}=\lambda_{k}^{\prime} e_{k}^{\prime} \in \mathrm{H}$, i. e. $e_{k}^{\prime} \in \mathrm{H}, k \in \mathbb{N}$. For the quadratic form we obtain

$$
\begin{aligned}
Q(f) & =\sum_{k=1}^{\infty} \lambda_{k}^{\prime}\left|\left\langle e_{k}^{\prime}, f\right\rangle\right|^{2}=\sum_{k=1}^{\infty} \lambda_{k}^{\prime}\left|\sigma\left(e_{k}^{\prime}, J f\right)+\mathrm{i} \sigma\left(e_{k}^{\prime}, f\right)\right|^{2} \\
& =\sum_{k=1}^{\infty} \lambda_{k}^{\prime} \sigma\left(J e_{k}^{\prime}, f\right)^{2}+\sum_{k=1}^{\infty} \lambda_{k}^{\prime} \sigma\left(e_{k}^{\prime}, f\right)^{2}=\sum_{k=1}^{\infty} \lambda_{k} \sigma\left(e_{k}, f\right)^{2}, \quad f \in \mathrm{S},
\end{aligned}
$$

with $\lambda_{2 k}=\lambda_{2 k-1}=\lambda_{k}^{\prime}$, and $e_{2 k}=e_{k}^{\prime}, e_{2 k-1}=J e_{k}^{\prime}, k \in \mathbb{N}$. Thus the quadratic form has been diagonalized in the symplectic basis $\left\{e_{k}\right\}_{k \in \mathbb{N}}$. The rest of the proof proceeds as in Proposition 4.20.

It is worth noting that in [131] generators of Gaussian semigroups on representations of $\mathfrak{A}(\mathrm{S})$ were studied, but in a different setting and under somewhat more restrictive assumptions. Note also that the proofs in that paper contain some gaps [132], one of which is filled by our Corollary B.5. 


\subsubsection{The Poisson Case}

Let $\nu \in \mathscr{M}_{\mathrm{b}}^{+}(\hat{\mathrm{S}})$ and suppose that $t \mapsto(\mathcal{F} \nu)\left(S_{t}(f)\right)$ is measurable and locally integrable for all $f \in \mathrm{S}$. It was shown in Proposition 4.5 that

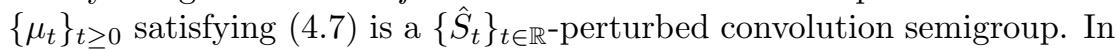
this case we say that $\left\{\mu_{t}\right\}_{t \geq 0}$ and the corresponding semigroup $\left\{\tau_{t}\right\}_{t \geq 0}$ on $\mathfrak{A}(\mathrm{S})$ given by Theorem 4.8 are of Poisson type.

From the above definitions we have

$$
\left(\mathcal{F} \nu_{t}\right)(f)=\int_{0}^{t} \int_{\hat{\mathrm{S}}} \chi(f) \mathrm{d}\left[\left(\hat{S}_{r}\right)_{*} \nu\right](\chi) \mathrm{d} r=\int_{0}^{t}(\mathcal{F} \nu)\left(S_{r}(f)\right) \mathrm{d} r
$$

and recall (4.7),

$$
\left(\mathcal{F} \mu_{t}\right)(f)=\mathrm{e}^{-t\|\nu\|} \exp \left(\left(\mathcal{F} \nu_{t}\right)(f)\right), \quad f \in \mathrm{S}, t \geq 0 .
$$

We see that if $f \mapsto(\mathcal{F} \nu)(f)$ is $\sigma(\mathrm{S}, \mathrm{S})$ continuous then condition E1 is satisfied because $S_{t}$ is symplectic; moreover, E 2 is satisfied as well. Now it follows from Theorem D.2 that there exists a bounded measure $\nu_{0}$ on $\sigma(\mathscr{U} \mathrm{s})$ such that

$$
(\mathcal{F} \nu)(f)=\int_{\mathrm{S}} \mathrm{e}^{\mathrm{i} \sigma(f, g)} \mathrm{d} \nu_{0}(g), \quad f \in \mathrm{S} .
$$

Then we can formulate the following result.

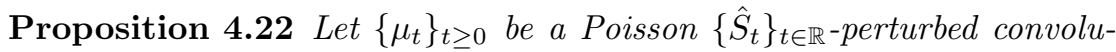
tion semigroup as above and assume that $f \mapsto(\mathcal{F} \nu)(f)$ is $\tau_{\mathrm{n}}(\mathrm{S}, \sigma(\mathrm{S}, \mathrm{S}))$ continuous. Moreover, assume that E 3-E 6 are satisfied for some state $\omega$ on $\mathfrak{A}(\mathrm{S})$. Then the semigroup $\left\{\tau_{t}\right\}_{t \geq 0}$ on $\mathfrak{A}(\mathrm{S})$ given by Theorem 4.8 is $\omega$-covariant and the generator $Z$ of the corresponding quantum dynamical semigroup $\left\{T_{t}\right\}_{t \geq 0}$ on $\mathfrak{M}$ induced by the covariance condition (B.9) is given by

$$
Z x=\delta(x)+L_{\mathrm{P}}(x), \quad x \in C,
$$

where

$$
L_{\mathrm{P}}(x)=\int_{\mathrm{S}}\left[W_{\omega}(g) x W_{\omega}(g)^{*}-x\right] \mathrm{d} \nu_{0}(g), \quad x \in \mathfrak{M},
$$

and $L_{\mathrm{P}}$ is a bounded normal operator. 
Proof. By the preceding remarks E 1-E 6 are satisfied. For $f \in \mathrm{S}$ and $\zeta \in \mathrm{C}_{\mathrm{c}}^{\infty}(] 0, \infty[)$ let $x_{\zeta}=\int_{0}^{\infty} \zeta(t) T_{t}\left(W_{\omega}(f)\right) \mathrm{d} t \in C$. From (4.29) and (4.30) we have, as in the proof of Proposition 4.20,

$$
\begin{aligned}
Z x_{\zeta} & =\int_{0}^{\infty} \zeta(t) \frac{\mathrm{d}}{\mathrm{d} t}\left[\Gamma_{t}(f) \beta_{t}\left(W_{\omega}(f)\right)\right] \mathrm{d} t \\
& =\delta\left(x_{\zeta}\right)+\int_{0}^{\infty} \zeta(t) \Gamma_{t}(f)\left[(\mathcal{F} \nu)\left(S_{t}(f)\right)-\|\nu\|\right] W_{\omega}\left(S_{t}(f)\right) \mathrm{d} t \\
& =\delta\left(x_{\zeta}\right)+\int_{0}^{\infty} \int_{\mathrm{S}} \zeta(t) \Gamma_{t}(f)\left[\mathrm{e}^{\mathrm{i} \sigma\left(S_{t}(f), g\right)}-1\right] W_{\omega}\left(S_{t}(f)\right) \mathrm{d} \nu_{0}(g) \mathrm{d} t \\
& =\delta\left(x_{\zeta}\right)+\int_{\mathrm{S}} \int_{0}^{\infty} \zeta(t) \Gamma_{t}(f)\left[W_{\omega}(g) W_{\omega}\left(S_{t}(f)\right) W_{\omega}(g)^{*}\right. \\
& \left.\left.=\delta\left(x_{\zeta}\right)+\int_{S}\left[W_{\omega}(g) x_{\zeta} W_{\omega}(g)^{*}-x_{\zeta}\right] \mathrm{d} \nu_{0}(g)\right)\right] \mathrm{d} t \mathrm{~d} \nu_{0}(g)
\end{aligned}
$$

where we have used (4.40) and (4.41), the Weyl relations, and Fubini's theorem. We conclude that (4.43) holds for all $x \in C$.

\subsubsection{The Dirac Case}

Let $\psi \in \mathrm{S}^{*}$ be a linear functional on $\mathrm{S}$ such that $t \mapsto \psi\left(S_{t}(f)\right)$ is measurable and locally integrable for all $f \in \mathrm{S}$. Define $\psi_{t}(f)=\int_{0}^{t} \psi\left(S_{r}(f)\right) \mathrm{d} r$ and the character $\chi_{t} \in \hat{\mathrm{S}}$ by $\chi_{t}(f)=\mathrm{e}^{\mathrm{i} \psi_{t}(f)}$. Then by Proposition 4.23 $\left\{\mu_{t}\right\}_{t \geq 0}$, where $\mu_{t}=\delta_{\chi_{t}}$, is a $\left\{\hat{S}_{t}\right\}_{t \in \mathbb{R}}$-perturbed convolution semigroup. We say that $\left\{\mu_{t}\right\}_{t \geq 0}$ and the corresponding semigroup $\left\{\tau_{t}\right\}_{t \geq 0}$ on $\mathfrak{A}(\mathrm{S})$ obtained by Theorem 4.8 are of Dirac type.

The map $f \mapsto\left(\mathcal{F} \mu_{t}\right)(f)=\chi_{t}(f)$ is $\sigma(\mathrm{S}, \mathrm{S})$ continuous if and only if $\psi \in \mathrm{S}$ since $S_{t}$ is symplectic, $t \geq 0$; in this case E 1 and $\mathrm{E} 2$ are satisfied.

Proposition 4.23 Let $\psi$ be a linear functional of the form $\psi(f)=\sigma(f, g)$ where $g \in \mathrm{S}$, and let $\left\{\mu_{t}\right\}_{t \geq 0}$ be the associated perturbed convolution semigroup of Dirac type. Suppose that $\omega$ is a regular state on $\mathfrak{A}(\mathrm{S})$ such that $\xi_{\omega} \in \operatorname{dom} \Phi_{\omega}(f)$ for all $f \in \mathrm{S}$ and such that $E 3-E 6$ are satisfied. Then the corresponding semigroup $\left\{\tau_{t}\right\}_{t \geq 0}$ given by Theorem 4.8 is $\omega$-covariant and the generator $Z$ of the quantum dynamical semigroup $\left\{T_{t}\right\}_{t \geq 0}$ on $\mathfrak{M}$ induced by the covariance condition (B.9) is given by

$$
Z x=\delta(x)+\mathrm{i}\left[\Phi_{\omega}(g), x\right], \quad x \in C .
$$


Proof. We proceed as in the proof of Proposition 4.20. For $f \in \mathrm{S}$ and $\zeta \in \mathrm{C}_{\mathrm{c}}^{\infty}(] 0, \infty[)$ let $x_{\zeta}=\int_{0}^{\infty} \zeta(t) T_{t}\left(W_{\omega}(f)\right) \mathrm{d} t \in C$, then

$$
\begin{aligned}
Z x_{\zeta} & =\int_{0}^{\infty} \zeta(t) \frac{\mathrm{d}}{\mathrm{d} t}\left[\Gamma_{t}(f) W_{\omega}\left(S_{t}(f)\right)\right] \mathrm{d} t \\
& =\delta\left(x_{\zeta}\right)+\mathrm{i} \int_{0}^{\infty} \zeta(t) \sigma\left(S_{t}(f), g\right) T_{t}\left(W_{\omega}(f)\right) \mathrm{d} t \\
& =\delta\left(x_{\zeta}\right)+\mathrm{i} \int_{0}^{\infty} \zeta(t)\left[\Phi_{\omega}(g), T_{t}\left(W_{\omega}(f)\right)\right] \mathrm{d} t \\
& =\delta\left(x_{\zeta}\right)+\mathrm{i}\left[\Phi_{\omega}(g), x_{\zeta}\right],
\end{aligned}
$$

where we have used (4.34). We conclude that (4.45) holds for all $x \in C$. $\square$

\subsection{Normal Invariant States}

In this section we discuss the existence of normal faithful invariant states for the semigroups obtained by Proposition 4.15. The notation of the previous section will be retained. We assume that the $\sigma(\mathrm{S}, \mathrm{S})$ topology satisfies the first axiom of countability and is separable; again this is, for example, the case if the symplectic space arises from a complex separable pre-Hilbert space in the canonical way. Under this assumption the $\sigma$ algebra $\sigma(\mathscr{U} \mathrm{S})$ is nothing else as the Borel $\sigma$-algebra $\mathscr{B}(\mathrm{S}, \sigma)$ of the $\sigma(\mathrm{S}, \mathrm{S})$ topology.

The invariant state will be constructed from a measure $\mu \in \mathscr{M}_{1}^{+}(\hat{\mathrm{S}})$ which satisfies the condition

$$
\mu=\mu_{t} *\left(\hat{S}_{t}\right)_{*} \mu, \quad t \geq 0,
$$

and with is such that the map $f \mapsto(\mathcal{F} \mu)(f)$ is $\sigma(\mathrm{S}, \mathrm{S})$ continuous. The first result gives a condition for the uniqueness of such a measure.

Lemma 4.24 Let $\mu \in \mathscr{M}_{1}^{+}(\hat{\mathrm{S}})$ be a measure satisfying (4.46). Moreover, assume that $f \mapsto(\mathcal{F} \mu)(f)$ is $\sigma(\mathrm{S}, \mathrm{S})$ continuous and

$$
\lim _{t \rightarrow \infty} S_{t}(f)=0
$$

in the $\sigma(\mathrm{S}, \mathrm{S})$ topology for any $f \in \mathrm{S}$. Then $\mu$ is uniquely determined and we have

$$
\lim _{t \rightarrow \infty} \mu_{t}=\mu
$$

in the weak topology (i. e. the $\sigma\left(\mathscr{M}_{\mathrm{b}}(\hat{\mathrm{S}}), \mathrm{C}_{\mathrm{b}}(\hat{\mathrm{S}})\right)$-topology). 
Proof. By the assumptions there is for any $f \in \mathrm{S}$,

$$
\lim _{t \rightarrow \infty}\left(\mathcal{F}\left(\hat{S}_{t}\right)_{*} \mu\right)(f)=\lim _{t \rightarrow \infty}(\mathcal{F} \mu)\left(S_{t}(f)\right)=1,
$$

and from (4.46) it follows that $(\mathcal{F} \mu)(f)=\left(\mathcal{F} \mu_{t}\right)(f) \cdot(\mathcal{F} \mu)\left(S_{t}(f)\right)$. Letting $t \rightarrow \infty$ gives $\lim _{t \rightarrow \infty}\left(\mathcal{F} \mu_{t}\right)(f)=(\mathcal{F} \mu)(f)$ uniformly on compact sets of $\mathrm{S}$ (i.e. pointwise), hence $\mu_{t} \rightarrow \mu$ weakly.

Proposition 4.25 Assume that the functions $\left\{\mathcal{F} \mu_{t}: t \geq 0\right\}$ are equicontinuous at 0 for the $\sigma(\mathrm{S}, \mathrm{S})$ topology, i. e. for every $\epsilon>0$ there exists a 0 -neighborhood $U$ of $\sigma(\mathrm{S}, \mathrm{S})$ such that $\left|1-\left(\mathcal{F} \mu_{t}\right)(f)\right|<\epsilon$ for all $t \geq 0$ and $f \in U$. Then there exists a measure $\mu \in \mathscr{M}_{1}^{+}(\hat{\mathrm{S}})$ satisfying (4.46) such that $f \mapsto(\mathcal{F} \mu)(f)$ is $\sigma(\mathrm{S}, \mathrm{S})$ continuous.

Proof. By Theorem D.2 there exist positive normalized measures $\nu_{t}$ on $\sigma(\mathscr{U} \mathrm{S})=\mathscr{B}(\mathrm{S}, \sigma)$ such that

$$
\left(\mathcal{F} \mu_{t}\right)(f)=\int_{\mathrm{S}} \mathrm{e}^{\mathrm{i} \sigma(f, g)} \mathrm{d} \nu_{t}(g)=\hat{\nu}_{t}(f)
$$

(cf. the proof of Proposition 4.15), where $\hat{\nu}_{t}$ denotes the Fourier transform of $\nu_{t}$ in $\mathscr{M}_{\mathrm{b}}(\mathrm{S}, \sigma(\mathrm{S}, \mathrm{S}))$. From the condition

$$
\left(\mathcal{F} \mu_{t+s}\right)(f)=\left(\mathcal{F} \mu_{t}\right)(f)\left(\mathcal{F} \mu_{s}\right)\left(S_{t}(f)\right)
$$

it follows that

$$
\nu_{t+s}=\nu_{t} *\left(S_{t}\right)_{*} \nu_{s}, \quad s, t \geq 0,
$$

where the convolution is taken in $\mathscr{M}_{\mathrm{b}}(\mathrm{S}, \sigma(\mathrm{S}, \mathrm{S}))$. From Corollary C.8 it follows that the Sazonov topology on $(\mathrm{S}, \sigma(\mathrm{S}, \mathrm{S})$ ) agrees with $\sigma(\mathrm{S}, \mathrm{S})$, and consequently the Sazonov on $(\mathrm{S}, \tau(\mathrm{S}, \mathrm{S}))$ is stronger than $\sigma(\mathrm{S}, \mathrm{S})$. Here $\tau(\mathrm{S}, \mathrm{S})$ denotes the Mackey topology. Hence it follows that $\left\{\hat{\nu}_{t}: t \geq\right.$ $0\}$ is also equicontinuous at 0 relative to $\tau_{\mathrm{n}}(\mathrm{S}, \tau(\mathrm{S}, \mathrm{S}))$ and so is $\operatorname{co}\left\{\hat{\nu}_{t}\right.$ : $t \geq 0\}=\left\{\hat{\nu}: \nu \in K_{0}\right\}$, where $K_{0}:=\operatorname{co}\left\{\nu_{t}: t \geq 0\right\}$. Now it follows from Theorem D.3 that $K_{0}$ is relatively weakly compact, thus $K=\bar{K}_{0}$ is weakly compact and consists of probability measures. Define the maps $\tau_{t}: \mathscr{M}_{\mathrm{b}}(\mathrm{S}) \longrightarrow \mathscr{M}_{\mathrm{b}}(\mathrm{S})$ by

$$
\rho \mapsto \tau_{t}(\rho)=\nu_{t} *\left(S_{t}\right)_{*} \rho, \quad t \geq 0 .
$$

Then

$$
\tau_{s} \circ \tau_{t}(\rho)=\nu_{s} *\left(S_{s}\right)_{*}\left(\nu_{t} *\left(S_{t}\right)_{*} \rho\right)
$$




$$
\begin{aligned}
& =\nu_{s} *\left(S_{s}\right)_{*} \nu_{t} *\left(S_{s+t}\right)_{*} \rho \\
& =\nu_{s+t} *\left(S_{s+t}\right)_{*} \rho=\tau_{s+t}(\rho), \quad s, t \geq 0,
\end{aligned}
$$

so $\left\{\tau_{t}\right\}_{t \geq 0}$ is a semigroup of linear contractions on $\mathscr{M}_{\mathrm{b}}(\mathrm{S}, \sigma(\mathrm{S}, \mathrm{S}))$. Since $\tau_{s}\left(\nu_{t}\right)=\nu_{s} *\left(S_{s}\right)_{*} \nu_{t}=\nu_{s+t}$ it follows that $\tau_{t}(K) \subseteq K$ for all $t \geq 0$. We now show that $K \ni \rho \mapsto \tau_{t}(\rho)$ is weakly continuous. Since $\left\{\hat{\nu}_{t}: t \geq 0\right\}$ is equicontinuous relative to $\sigma(\mathrm{S}, \mathrm{S})$ at 0 it follows that $\{\hat{\nu}: \nu \in K\}$ is so as well, hence by Theorem D.4 the set $K$ is uniformly tight. Thus if $\epsilon>0$ there exists a compact set $C \subseteq \mathrm{S}$ such that $\nu(\mathrm{S} \backslash C)<\epsilon$ for any $\nu \in K$. For $\zeta \in \mathrm{C}_{\mathrm{b}}(\mathrm{S}, \sigma(\mathrm{S}, \mathrm{S}))$, a measure $\rho \in K$, and a net $\left\{\nu_{i}\right\}_{i \in I} \subseteq K$ with $\nu_{i} \rightarrow \nu$ weakly we have

$$
\int_{\mathrm{S}} \zeta(f) \mathrm{d}\left(\rho * \nu_{i}\right)(f)=\int_{\mathrm{S}} \int_{\mathrm{S}} \zeta(f+g) \mathrm{d} \nu_{i}(f) \mathrm{d} \rho(g)=\int_{\mathrm{S}} \eta_{i}(g) \mathrm{d} \rho(g)
$$

with $\eta_{i}(g)=\int_{\mathrm{S}} \zeta(f+g) \mathrm{d} \nu_{i}(f)$. Hence

$$
\left|\eta_{i}\left(g_{1}\right)-\eta_{i}\left(g_{2}\right)\right| \leq 2 \epsilon\|\zeta\|_{\infty}+\sup _{f \in C}\left|\zeta\left(f+g_{1}\right)-\zeta\left(f+g_{2}\right)\right|,
$$

for any $i \in I$ where $g_{1}, g_{2} \in \mathrm{S}$. Now let $C_{1} \subseteq \mathrm{S}$ be any compact subset, then $C_{1}+C$ is compact and $\zeta$ is uniformly continuous on $C_{1}+C$. Thus there exists a 0 -neighborhood $U$ in S such that whenever $f, g \in C_{1}+C$, $f-g \in U$ it follows that $|\zeta(f)-\zeta(g)|<\epsilon$. In particular, if $g_{1}, g_{2} \in C_{1}$ and $g_{1}-g_{2} \in U$ then $\left|\zeta\left(f+g_{1}\right)-\zeta\left(f+g_{2}\right)\right|<\epsilon$ for $f \in C$, thus by (4.48)

$$
\left|\eta_{i}\left(g_{1}\right)-\eta_{i}\left(g_{2}\right)\right|<\epsilon\left(2\|\zeta\|_{\infty}+1\right)
$$

for any $i \in I$, i. e. $\left\{\eta_{i}\right\}_{i \in I}$ is equicontinuous on any compact set $C_{1} \subseteq \mathrm{S}$, and since $\eta_{i}(g) \rightarrow \eta(g)$ for all $g \in \mathrm{S}$, where $\eta(g)=\int_{\mathrm{S}} \zeta(f+g) \mathrm{d} \nu(f)$, the same is true for $\left\{\eta_{i}\right\}_{i \in I} \cup\{\eta\}$. Now by equicontinuity the topology of pointwise convergence and the compact open topology coincide on $\left\{\eta_{i}\right\}_{i \in I} \cup\{\eta\}$, hence $\eta_{i} \rightarrow \eta$ uniformly on compact subsets of S. Thus there is $i_{0} \in I$ such that $i \geq i_{0}$ implies $\left|\eta_{i}(g)-\eta(g)\right|<\epsilon$ for all $g \in C$, hence from (4.47)

$$
\begin{aligned}
\left|\int_{\mathrm{S}} \zeta(f) \mathrm{d}\left(\rho * \nu_{i}\right)(f)-\int_{\mathrm{S}} \zeta(f) \mathrm{d}(\rho * \nu)(f)\right| \\
\leq \sup _{g \in C}\left|\eta_{i}(g)-\eta(g)\right|+2\|\zeta\|_{\infty} \rho(\mathrm{S} \backslash C) \\
<\epsilon\left(1+2\|\zeta\|_{\infty}\right)
\end{aligned}
$$

if $i \geq i_{0}$. So $K \ni \nu \mapsto \rho * \nu, \rho \in K$, is weakly continuous and we conclude that $K \ni \rho \mapsto \tau_{t}(\rho)$ is so as well for any $t \geq 0$. Now by the MarkovKakutani theorem there exists a measure $\nu \in K$ such that $\tau_{t}(\nu)=\nu$ 
for all $t \geq 0$, and using Bochner's theorem $\hat{\nu}=\mathcal{F} \mu$ defines a measure $\mu \in \mathscr{M}_{1}^{+}(\hat{\mathrm{S}})$ with the required properties.

The next theorem shows how a measure satisfying (4.46) can be used to construct a normal faithful invariant state for the semigroups obtained from Proposition 4.15.

Theorem 4.26 Suppose that the conditions of Proposition 4.15 are satisfied. Assume the existence of a faithful normal invariant state $\varphi$ for the automorphism group $\left\{\beta_{t}\right\}_{t \in \mathbb{R}}$ of condition $E 4$ (see equation (4.22)) and the existence of a measure $\mu$ satisfying (4.46) such that $f \mapsto(\mathcal{F} \mu)(f)$ is $\sigma(\mathrm{S}, \mathrm{S})$ continuous. Then there exists a faithful normal invariant state for the semigroup $\left\{T_{t}\right\}_{t \geq 0}$.

Proof. Under the assumptions of the theorem there exists a probability measure $\nu$ on $\mathscr{B}(\mathrm{S}, \sigma)$ such that

$$
T(x)=\int_{\mathbf{S}} W_{\omega}(g) x W_{\omega}(g)^{*} \mathrm{~d} \nu(g), \quad x \in \mathfrak{M},
$$

defines a normal positive unital map $T$ such that

$$
T\left(W_{\omega}(f)\right)=(\mathcal{F} \mu)(f) W_{\omega}(f)
$$

for all $f \in \mathrm{S}$. This map is faithful since $T\left(x^{*} x\right)=0$ implies

$$
W_{\omega}(g) x^{*} x W_{\omega}(g)^{*}=0 \quad \nu \text {-almost everywhere, }
$$

i. e. $x^{*} x=0$, hence $\varphi \circ T$ is a faithful normal state. Now we have

$$
\begin{aligned}
T \circ T_{t}\left(W_{\omega}(f)\right) & =\left(\mathcal{F} \mu_{t}\right)(f) \cdot(\mathcal{F} \mu)\left(S_{t}(f)\right) \cdot W_{\omega}\left(S_{t}(f)\right) \\
& =\mathcal{F}\left(\mu_{t} *\left(\hat{S}_{t}\right)_{*} \mu\right)(f) \cdot W_{\omega}\left(S_{t}(f)\right) \\
& =(\mathcal{F} \mu)(f) \cdot \beta_{t}\left(W_{\omega}(f)\right)
\end{aligned}
$$

for all $f \in \mathrm{S}$ and $t \geq 0$, from which follows that $\varphi \circ T\left(T_{t}(x)\right)=\varphi \circ T(x)$ for all $x \in \mathfrak{M}$, i. e. $\varphi \circ T$ is $T_{t}$-invariant.

\subsection{Detailed Balance for Quasifree Semigroups}

\subsubsection{General Condition}

In the present section we shall give a sufficient criterion for a quasifree semigroup $\left\{\tau_{t}\right\}_{t \geq 0}$ of positive unital and contractive maps on the CCR 
algebra $\mathfrak{A}(\mathrm{S})$ over a symplectic space $(\mathrm{S}, \sigma)$ to satisfy detailed balance II (Definition 2.21) with respect to some state $\omega \in \mathscr{S}(\mathfrak{A}(\mathrm{S}, \sigma))$. Suppose that $\left\{\tau_{t}\right\}_{t \geq 0}$ is given by

$$
\tau_{t}(W(f))=\Gamma_{t}(f) W\left(S_{t}(f)\right), \quad f \in \mathrm{S}, t \geq 0,
$$

where $\left\{S_{t}\right\}_{t \geq 0}$ is a semigroup of linear maps on S. If detailed balance II is satisfied with respect to a state $\omega \in \mathscr{S}(\mathfrak{A}(\mathrm{S}, \sigma))$, and if the semigroup $\left\{\tau_{t}^{\beta}\right\}_{t \geq 0}$ is quasifree as well, i. e.

$$
\tau_{t}^{\beta}(W(f))=\Gamma_{t}^{\beta}(f) W\left(S_{t}^{\beta}(f)\right), \quad f \in \mathrm{S}, t \geq 0,
$$

then the condition (2.34) for detailed balance II reads

$$
\begin{aligned}
\mathrm{e}^{-\mathrm{i} \sigma\left(f, S_{t}(g)\right) / 2} \Gamma_{t}(f) \phi_{\omega}(f+ & \left.S_{t}(g)\right) \\
& =\mathrm{e}^{-\mathrm{i} \sigma\left(S_{t}^{\beta}(f), g\right) / 2} \Gamma_{t}^{\beta}(f) \phi_{\omega}\left(S_{t}^{\beta}(f)+g\right),
\end{aligned}
$$

for all $f, g \in \mathrm{S}$ and $t \geq 0$. Here $\phi_{\omega}$ is the generating functional of the state $\omega$. Conversely, if there are two quasifree semigroups $\left\{\tau_{t}\right\}_{t \geq 0}$ and $\left\{\tau_{t}^{\beta}\right\}_{t \geq 0}$ satisfying (4.50) and (4.51), and if (4.52) holds, then $\left\{\tau_{t}\right\}_{t \geq 0}$ satisfies detailed balance II with respect to $\omega$.

\subsubsection{Example}

In this section we give a simple mathematical example of a semigroup on the CCR algebra which satisfies detailed balance II, hence it extends to a dynamical semigroup on $\mathfrak{M}$ (compare Proposition 2.27), and by Theorem 3.6 it displays also decoherence. Let $(\mathrm{S}, \sigma)$ be a symplectic space with a nondegenerate symplectic form $\sigma$. We consider a quasifree state $\omega$ on $\mathfrak{A}(\mathrm{S}, \sigma)$ defined by $s \in \mathscr{A}$, such that $\phi_{\omega}=\omega(W(f))=\mathrm{e}^{-s(f, f) / 4}$ (see Section A.2.2 for an explanation of the notation). We shall write

$$
Q(f)=s(f, f), \quad f \in \mathrm{S},
$$

then $Q$ is a positive quadratic form on $\omega$. Fix $\lambda>0$ and define the semigroup $\left\{S_{t}\right\}_{t \geq 0}$ by $S_{t}(f)=\mathrm{e}^{-\lambda t} f$ for any $f \in \mathrm{S}$, and let $S_{t}^{\beta}=S_{t}=S_{t}^{+}$; then their generator $Z$ satisfies $Z f=-\lambda f$. Moreover, we let

$$
\psi(f)=-\frac{\lambda}{2} Q(f), \quad f \in \mathrm{S}
$$

and

$$
\Gamma_{t}(f)=\Gamma_{t}^{\beta}(f)=\exp \left(\int_{0}^{t} \psi\left(S_{r}(f)\right) \mathrm{d} r\right)
$$




$$
=\exp \left(-\frac{1}{4} Q(f)\left(1-\mathrm{e}^{-2 \lambda t}\right)\right), \quad f \in \mathrm{S}, t \geq 0 .
$$

Then condition (4.52) becomes

$$
\begin{aligned}
\exp \left(-\frac{1}{4} Q(g)\left(1-\mathrm{e}^{-2 \lambda t}\right)\right) \exp \left(-\frac{1}{4} Q\left(f+\mathrm{e}^{-\lambda t} g\right)\right) \\
=\exp \left(-\frac{1}{4} Q(f)\left(1-\mathrm{e}^{-2 \lambda t}\right)\right) \exp \left(-\frac{1}{4} Q\left(\mathrm{e}^{-\lambda t} f+g\right)\right),
\end{aligned}
$$

or

$$
\left(1-\mathrm{e}^{-2 \lambda t}\right) Q(g)+Q\left(f+\mathrm{e}^{-\lambda t} g\right)=\left(1-\mathrm{e}^{-2 \lambda t}\right) Q(f)+Q\left(\mathrm{e}^{-\lambda t} f+g\right)
$$

for all $f, g \in \mathrm{S}$. Substituting $s(f, f)$ for $Q(f)$ and multiplying out we see that this condition is satisfied. Hence if $\left\{\tau_{t}\right\}_{t \geq 0}$ is defined by

$$
\tau_{t}(W(f))=\Gamma_{t}(f) W\left(S_{t}(f)\right), \quad f \in \mathbf{S}, t \geq 0
$$

on $\mathfrak{A}_{0}(\mathrm{~S}, \sigma)$, and if it extends to a completely positive contractive semigroup on $\mathfrak{A}(\mathrm{S}, \sigma)$, then the extension will satisfy detailed balance II and $\tau_{t}=\tau_{t}^{\beta}$ for all $t \geq 0$. To prove that the extension exists we use Proposition 4.13. From (A.25) we have

$$
|\sigma(f, g)| \leq \sqrt{s(f, f)} \sqrt{s(g, g)} \leq \frac{1}{2} s(f, f)+\frac{1}{2} s(g, g)
$$

for all $f, g \in \mathrm{S}$, hence

$$
\begin{aligned}
\sigma(Z f, g)+\sigma(f, Z g) & =-2 \lambda \sigma(f, g) \\
& \leq \lambda(Q(f)+Q(g)) \\
& =-2 \psi(f)-2 \psi(g),
\end{aligned}
$$

and by Proposition 4.13 the semigroup $\left\{\tau_{t}\right\}_{t \geq 0}$ extends to a completely positive contractive semigroup on $\mathfrak{A}(\mathrm{S}, \sigma)$, satisfying detailed balance with respect to $\omega$.

Now we introduce additional assumptions on the state $\omega$ : We assume $\omega$ to be separating (for example a KMS state). Moreover, if $\left(\pi_{\omega}, \xi_{\omega}, \mathscr{H}_{\omega}\right)$ is its GNS representation we assume that $\mathscr{H}_{\omega}$ is separable. Next we assume that the map

$$
\mathbb{R}^{+} \ni t \mapsto \pi_{\omega}\left(W\left(\mathrm{e}^{-\lambda t} f\right)\right)
$$

is continuous with respect to the weak operator topology for any $f \in \mathrm{S}$. Then it follows that $t \mapsto \pi_{\omega}\left(\tau_{t}(W(f))\right)=\Gamma_{t}(f) \pi_{\omega}\left(W\left(S_{t}(f)\right)\right)$ is continuous for any $f \in \mathrm{S}$, hence $t \mapsto\left(\pi_{\omega}\left(\tau_{t}(x)\right)\right)$ is continuous for any $\mathfrak{B}=\operatorname{lin}\left\{\pi_{\omega}(W(f)): f \in \mathrm{S}\right\}$. By detailed balance the state $\omega$ is invariant under $\left\{\tau_{t}\right\}_{t \geq 0}$, hence we see that by the method of Theorem B.7 the 
semigroup $\left\{\tau_{t}\right\}_{t \geq 0}$ is $\omega$-covariant. Hence there exists a semigroup $\left\{T_{t}\right\}_{t \geq 0}$ of completely positive normal contractive maps on $\mathfrak{M}=\pi_{\omega}(\mathfrak{A}(\mathrm{S}, \sigma))$ such that (B.9) holds, and since $\mathfrak{B} \subseteq \mathfrak{M}$ is a nondegenerate ${ }^{*}$-algebra such that $\mathfrak{B}^{\prime \prime}=\mathfrak{M}$ it follows from Corollary B.5 that $t \mapsto T_{t}(x)$ is ultraweakly continuous for any $x \in \mathfrak{M}$; hence $\left\{T_{t}\right\}_{t \geq 0}$ is a quantum dynamical semigroup. Let $\hat{\omega}$ be the extension of $\omega$ to a normal state on $\mathfrak{M}$, i. e. $\hat{\omega}(x)=\left\langle\xi_{\omega}, x \xi_{\omega}\right\rangle$ for $x \in \mathfrak{M}$. From Proposition 2.27 we see that $\left\{T_{t}\right\}_{t \geq 0}$ also satisfies detailed balance II with respect to $\hat{\omega}$. Then by Theorem 3.6 the semigroup $\left\{T_{t}\right\}_{t \geq 0}$ displays decoherence, i. e. the splitting $\mathfrak{M}=\mathfrak{M}_{1} \oplus \mathfrak{M}_{2}$ of Definition 3.1 exists. Moreover, there exists a normal conditional expectation $E$ onto $\mathfrak{M}_{1}$. Our next task is to identify the algebra of effective observables $\mathfrak{M}_{1}$.

Our first observation is that, since $T_{t}=T_{t}^{\beta}$, we have $\mathfrak{M}_{1}=\operatorname{fix}\left\{T_{t}\right\}_{t \geq 0}$ as a result of Proposition 2.33. Moreover, as a result of Theorem 3.6 resp. Theorem 2.3, or Corollary 1.27, there exists a normal conditional expectation $E$ onto $\mathfrak{M}_{1}=\operatorname{fix}\left\{T_{t}\right\}_{t \geq 0}$ such that $\hat{\omega} \circ E=\hat{\omega}$, i. e. $\left\{T_{t}\right\}_{t \geq 0}$ is mean ergodic. Now let $x=\sum_{i=1}^{n} \lambda_{i} W_{\omega}\left(f_{i}\right) \in \mathfrak{B}$, where $\pi_{\omega}(W(f))=$ $W_{\omega}(f)$, then

$$
\begin{aligned}
\lim _{t \rightarrow \infty} T_{t}(x) & =\lim _{t \rightarrow \infty} \sum_{i=1}^{n} \lambda_{i} \exp \left(-\frac{1}{4} Q\left(f_{i}\right)\left(1-\mathrm{e}^{-2 \lambda t}\right)\right) W_{\omega}\left(\mathrm{e}^{-\lambda t} f_{i}\right) \\
& =\sum_{i=1}^{n} \lambda_{i} \mathrm{e}^{-Q\left(f_{i}\right) / 4} \mathbb{1}=\hat{\omega}(x) \mathbb{1}
\end{aligned}
$$

for all $x \in \mathfrak{B}$. Then we have $E(x)=\hat{\omega}(x) \mathbb{1}$ for all $x \in \mathfrak{B}$ by a similar reasoning as in the proof of Proposition 2.36, hence $E=\omega(\cdot) \mathbb{1}$. We conclude that $\mathfrak{M}_{1}=\mathbb{C} \mathbb{1}$.

Thus we have shown that the construted semigroup displays decoherence and is moreover ergodic, compare Section 3.2.3. Clearly, this example is simple and of a purely mathematical nature. However, our method employed has the advantage that $(\mathrm{S}, \sigma)$ as well as the quasifree state $\omega$ are arbitrary, hence for suitable choices of $S$ and $s \in \mathscr{A}$ the von Neumann algebra $\mathfrak{M}$ can be of arbitrary type, in particular of III. 


\section{Chapter 5.}

\section{Conclusion and Open Problems}

\subsection{Conclusion}

There are two main aspects in this work: The first is the construction of quasifree completely positive semigroups on the CCR algebra and their extension to quantum dynamical semigroups on representations of the CCR algebra. The other aspect are some general theorems for the existence of the decoherence effect in the algebraic formulation if the system obeys a Markovian time evolution. Moreover, we attempted to explain the significance of the algebraic approach in the discussion of decoherence and its outstanding suitability for the discussion of foundational problems of quantum physics.

To this end we start in Section 1.1 from the notions preparation and measurement, from which the algebraic formulation of classical and quantum can be deduced with quite strict logic. Although we have not given complete arguments, we hope that the main ideas can be appreciated. In Section 1.2 we briefly explain the idea of quantum probability in the language of $\mathrm{C}^{*}$ - and von Neumann algebras. It is shown in which way quantum probability generalizes classical probability and how the algebraic formulation of physics can be understood in terms of quantum probability. Next it is explained how quantum and classical systems fit in this framework, and to which mathematical properties the quantum peculiarities are related. The following Section 1.3 introduces the description of open systems and their dynamics in the algebraic formulation which is very important in the present work. Section 1.4 introduces some mathematical results concerning fixed points and invariant states of open systems dynamics. The results here are mostly well known, except Proposition 1.31 which, in its present form as well as its proof, seem to be new.

Chapter 2 introduces the main technical tools for the discussion of decoherence in the algebraic framework. Section 2.2 discusses the isometricsweeping splitting, the proof of Theorem 2.6 is new. Moreover, we give a 
version of the Jacobs-de Leeuw-Glicksberg splitting and discuss it in the case of von Neumann algebras. Section 2.2.3 gives an illustrative example for its application to decoherence. Next we discuss a notion of noncommutative detailed balance with respect to a faithful normal state and in Theorem 2.29 we characterize it in terms of the modular group with respect to that state. Although some implications of Theorem 2.29 can be concluded from the literature (see the remarks on page 74), it never seems to have appeared in the present form before. Some sufficient conditions for detailed balance are given. Moreover, using the isometric-sweeping splitting and Theorem 2.29 we generalize a result of W. Majewski concerning the approach to equilibrium in the case of semigroup dynamics.

In Chapter 3 the concept of decoherence is introduced as a property of the irreversible dynamics of an open system; following the work of $\mathrm{Ph}$. Blanchard and R. Olkiewicz a definition is given in terms of the algebraic framework. We then apply our results obtained in Chapter 2 to this definition and obtain a number of sufficient conditions for the existence of the decoherence effect in the case of semigroup dynamics in Section 3.2.1. Here we exploit, in particular, the isometric sweeping and Jacobs-de Leeuw-Glicksberg splitting. We conclude from Theorem 2.29 that detailed balance implies decoherence; this observation appears here for the first time.

Chapter 4 concerns the second aspect of this work. Here we considered quasifree completely positive dynamical semigroups on the CCR algebra constructed by means of perturbed convolution semigroups. We discussed under which conditions a perturbed convolution semigroup induces a semigroup on the CCR algebra. Our most comprehensive result, which goes even beyond the framework of perturbed convolution semigroups, is Theorem 4.10, which is new and appears here for the first time. It characterizes the extendability of a class of semigroups, defined on the linear hull of all Weyl operators, to the CCR algebra. Proposition 4.13 shows how this theorem is applied. Moreover, we provide in Section 4.3 a comprehensive discussion of perturbed convolution semigroups on a symplectic space.

Next in Section 4.4, sufficient conditions (there labeled E 1-E 6) for the extendability of a class of semigroups on the CCR algebra to quantum dynamical semigroups on representations of the CCR algebra have been given (Proposition 4.15). The class semigroups on the CCR algebra for to which our extension method applies those induced by perturbed convolution semigroups such that $\left\{S_{t}\right\}_{t \in \mathbb{R}}$ is a symplectic group. We heavily rely on the material in Appendix B; the main results, Theorem B.4 and Theorem B.7, are new and apply to general quantum dynamical semigroups. Since nuclear and Sazonov topologies as well as cylindrical measures play 
an important role in the proof of the proof of Proposition 4.15, Appendix C collects some results concerning nuclear and Sazonov topologies and their connection, and Appendix D collects necessary results about cylindrical measures. The necessity and physical significance of this extension to quantum dynamical semigroups has been explained in Sections 1.2.4 and 1.3.2. For the quantum dynamical semigroups thus obtained (acting on von Neumann algebras corresponding to the representations) we have calculated the generators in Section 4.5 for the case Gaussian, Poisson, and Dirac perturbed convolution semigroups. The existence of the extension, Proposition 4.15 and Theorem B.7 as well as the calculation of the generators, will appear in [50]. Section 4.6 discusses the existence of normal invariant states for the quantum dynamical semigroups obtained by Proposition 4.15, a sufficient condition for the existence of such a state is given in terms of the perturbed convolution semigroup. However, this condition cannot be satisfied if the free evolution $\left\{S_{t}\right\}_{t \in \mathbb{R}}$ is required to be a symplectic group rather than a semigroup, so at present Theorem 4.26 is vacuous in the sense that does not apply to the semigroups we are able to control at the moment. But we expect the methods of Section 4.6 to become useful as soon as we achieve the extension of a more general class of semigroups than by means of Proposition 4.15. Finally in Section 4.7.2 we provide a first simple mathematical example of a quantum dynamical semigroup which shows decoherence.

\subsection{Open Problems}

Here we mention some open problems related to this work whose study seems worthwhile.

\section{Characterization of Detailed Balance}

The importance of detailed balance II (Definition 2.21) for quantum dynamical semigroups in the discussion of decoherence has been shown in Section 3.2.1. For this reason it would be interesting to have a characterization of detailed balance II for a quantum dynamical semigroup in terms of its generator. Consider a uniformly continuous quantum dynamical semigroup on a von Neumann algebra, then its generator is in Lindblad form (2.29). What are necessary and sufficient conditions on the operators $H$ and $A_{\omega}$ for the semigroup to satisfy detailed balance II with respect to a given faithful normal state? This question has been studied in $[73,36]$ 
but with a slightly different (more restrictive) notion of detailed balance than Definition 2.21.

\section{Generalization of Proposition 4.15}

Proposition 4.15 gives sufficient conditions for a semigroup on the CCR algebra, which is induced by a perturbed convolution semigroup, to be covariant with respect to a state $\omega$, i. e. to extend to a quantum dynamical semigroup on the von Neumann algebra corresponding to GNS representation of $\omega$. A major restriction is that $\left\{S_{t}\right\}_{t \in \mathbb{R}}$ is assumed to be a symplectic group, and it would be important to remove it. This generalization would include a number of interesting semigroups in our framework, which should be of interest in quantum optics. A possible approach is the following: Suppose that $\omega$ is a separating quasifree state on the CCR algebra $\mathfrak{A}(\mathrm{S}, \sigma)$, and let $\left\{\tau_{t}\right\}_{t \geq 0}$ be induced by a $\left\{\hat{S}_{t}\right\}_{t \geq 0}$-perturbed convolution semigroup $\left\{\mu_{t}\right\}_{t \geq 0}$. Then according to Theorem B.7 a semigroup $\left\{\tau_{t}\right\}_{t \geq 0}$ is $\omega$-covariant provided $\omega$ and $\omega \circ \tau_{t}$ are quasi-equivalent for all $t \geq 0$. If $\omega \circ \tau_{t}$ is quasifree as well, then one can use the characterization of quasi-equivalence of quasifree states given in [130] to directly verify that $\omega$ and $\omega \circ \tau_{t}$ are quasi-equivalent under appropriate conditions imposed upon $\left\{\hat{S}_{t}\right\}_{t \geq 0}$ and $\left\{\mu_{t}\right\}_{t \geq 0}$.

\section{A General Method to Construct Semigroups Satisfying Detailed Balance II}

At the beginning of Chapter 4 we have already commented about the difficulty to construct quantum dynamical semigroups on von Neumann algebras and the lack of examples. In this work we have established a class of quantum dynamical semigroups on representations of the CCR algebra. However, we may also ask if there is a general method to construct quantum dynamical semigroups on an arbitrary von Neumann algebra such that they automatically satisfy detailed balance II with respect to a faithful normal state, for then they automatically display decoherence. One might proceed as follows.

Suppose that $\mathfrak{M}$ is a von Neumann algebra acting on $\mathscr{H}$, and that $\xi_{0} \in \mathscr{H}$ is a normalized cyclic and separating vector for $\mathfrak{M}$. We define the real subspace

$$
\mathscr{H}_{\mathrm{r}}=\overline{\mathfrak{M}^{\mathrm{sa}} \xi_{0}} \subseteq \mathscr{H}
$$

together with the inner product $\langle\xi, \eta\rangle_{\mathrm{r}}=\operatorname{Re}\langle\xi, \eta\rangle, \xi, \eta \in \mathscr{H}$, the closed real subspace $\mathscr{H}_{\mathrm{r}}$ becomes a real Hilbert space. Moreover, we define the 
subset

$$
\mathscr{P}=\overline{\mathfrak{M}^{+} \xi_{0}}
$$

Then $\mathscr{P} \subseteq \mathscr{H}_{\mathrm{r}}$ is a cone, i. e. $\xi, \eta \in \mathscr{P}$ implies $\xi+\eta \in \mathscr{P}$ and $\lambda \geq 0$ and $\xi \in \mathscr{P}$ implies $\lambda \xi \in \mathscr{P}$. Its dual cone is given by

$$
\mathscr{P}^{\vee}=\{\xi \in \mathscr{H}:\langle\xi, \eta\rangle \geq 0 \forall \eta \in \mathscr{H}\}=\overline{\mathfrak{M}^{\prime+} \xi_{0}} .
$$

Then we have the following result.

Lemma 5.1 Suppose that $\hat{T}$ is a linear operator on $\mathscr{H}$ such that $\mathfrak{M} \xi_{0} \subseteq$ $\operatorname{dom} \hat{T}, \mathfrak{M}^{\prime} \xi_{0} \subseteq \operatorname{dom} \hat{T}^{*}$, and assume that $\hat{T}\left(\mathfrak{M}^{+} \xi_{0}\right) \subseteq \mathscr{P}$ as well as $\hat{T}\left(\xi_{0}\right)=\xi_{0}$. Then there exists a normal positive linear unital map $T$ : $\mathfrak{M} \longrightarrow \mathfrak{M}$ such that $T(x) \xi_{0}=\hat{T}\left(x \xi_{0}\right)$ for all $x \in \mathfrak{M}$.

The proof ${ }^{1}$ is similar to the proof of Theorem 2.29. Moreover, we can establish the following.

Lemma 5.2 The cone $\mathscr{P}$ has the following properties:

1. $\mathscr{P} \cap(-\mathscr{P})=\{0\}$.

2. For every $\xi \in \mathscr{H}_{\mathrm{r}}$ there exist $\xi_{1}, \xi_{2} \in \mathscr{P}$ such that $\xi=\xi_{1}-\xi_{2}$ and $\xi_{1} \perp \xi_{2}$.

3. The cone $\mathscr{P}$ is self-dual in $\mathscr{H}_{\mathrm{r}}$, i. e. $\mathscr{P}=\mathscr{P} \diamond$, where $\mathscr{P} \diamond=\{\xi \in$ $\left.\mathscr{H}_{\mathrm{r}}:\langle\xi, \eta\rangle_{\mathrm{r}} \geq 0 \forall \eta \in \mathscr{P}\right\}$ denotes its dual cone in $\mathscr{H}_{\mathrm{r}}$.

To construct dynamical semigroups on $\mathfrak{M}$ satisfying detailed balance II one might be able to proceed as follows: One starts with a strongly continuous semigroup $\left\{\hat{T}_{t}\right\}_{t \geq 0}$ of (real) linear contractive operators on $\mathscr{H}_{\mathrm{r}}$ such that $\hat{T}_{t}\left(\xi_{0}\right)=\xi_{0}$ and such that $\hat{T}_{t}(\mathscr{P}) \subseteq \mathscr{P}$ for all $t \geq 0$ (i. e. $\left\{\hat{T}_{t}\right\}_{t \geq 0}$ is positive with respect to the order induced by $\mathscr{P})$. Since $\mathscr{H}_{\mathrm{r}}$ is a real Hilbert space and $\mathscr{P}$ is a self-dual cone in $\mathscr{H}_{\mathrm{r}}$ (in particular this entails that $\mathscr{P}$ is a normal cone), there are some powerful Hille-Yosida type results for positive semigroups on $\mathscr{H}_{\mathrm{r}}$ available in the literature $[125,39,115]$ which allow the construction of positive semigroups on $\mathscr{H}_{\mathrm{r}}$. Now given such a semigroup on $\mathscr{H}_{\mathrm{r}}$ the idea is to extend each $\hat{T}_{t}$ to a complex linear operator on $\mathscr{H}$ such that the condition of Lemma 5.1 is satisfied; this will lead to a positive semigroup $\left\{T_{t}\right\}_{t \geq 0}$ on $\mathfrak{M}$. Imposing a further condition on the operators $\hat{T}_{t}$ and using the methods in the proof of Theorem 2.29 we may be able to construct semigroups $\left\{T_{t}\right\}_{t \geq 0}$ in this way which satisfy detailed balance II.

\footnotetext{
${ }^{1}$ Unpublished notes by the author.
} 


\section{Physical Models}

A physical model in which a subclass of the quasifree dynamical semigroups constructed in Chapter 4 arise is missing, see however [72] for some results in this direction. More models with a clear physical interpretation which display decoherence in our sense would be welcome. Moreover, a generalization of the example in Section 4.7.2 to obtain a more general $\left\{S_{t}\right\}_{t \geq 0}$ would be desirable. 


\section{Appendix A.}

\section{The CCR Algebra}

In this appendix we introduce the algebra of the canonical commutation relations, in short CCR algebra. It is the $\mathrm{C}^{*}$-algebra used to describe bosonic quantum systems (according to the spin-statistics theorem of relativistic quantum field theory). It has the fundamental property that to every representation of the canonical representation relations (in Weyl form) there corresponds a *-representation of the CCR algebra. It was introduced in [101] and further studied in [120, 103]; since then it has generated a vast amount of literature. The style of our presentation follows mainly [101, 103].

\section{A.1. Definition and Properties}

\section{A.1.1. Definition}

We will introduce the CCR algebra for not necessarily nondegenerate symplectic forms as in [103]. Let $\mathrm{S}$ be a symplectic group, i. e. an abelian group whose operation is denoted by + and its neutral element by 0 , endowed with a map $\sigma: \mathrm{S} \times \mathbf{S} \longrightarrow \mathbb{R}$ satisfying

$$
\begin{aligned}
\sigma(0, f) & =0, \\
\sigma(f, g) & =-\sigma(g, f), \\
\sigma(f+g, h) & =\sigma(f, h)+\sigma(g, h),
\end{aligned}
$$

for all $f, g, h \in \mathrm{S}$. We allow $\sigma$ to be degenerate; it will be called nondegenerate provided $\sigma(f, g)=0$ for all $f \in \mathrm{S}$ implies $g=0$. In most applications $\mathrm{S}$ will be a real vector space with addition playing the role of the above group operation, endowed with a symplectic form $\sigma$, i. e. a map satisfying (A.2) and (A.3), and additionally

$$
\sigma(\lambda f, g)=\lambda \sigma(f, g)
$$


for all $f, g \in \mathrm{S}$ and $\lambda \in \mathbb{R}$. Then we call the pair $(\mathrm{S}, \sigma)$ a symplectic space. Let $W(f), f \in \mathrm{S}$, be abstract symbols, called Weyl operators, and let $\mathfrak{A}_{0}(\mathrm{~S}, \sigma)$ be the set of all formal linear combinations of Weyl operators,

$$
\mathfrak{A}_{0}(\mathrm{~S}, \sigma)=\left\{\sum_{k=1}^{n} z_{k} W\left(f_{k}\right): z_{k} \in \mathbb{C}, f_{k} \in \mathrm{S}, n \in \mathbb{N}\right\} .
$$

Then the $W(f)$ are linearly independent and form a basis of the real vector space $\mathfrak{A}_{0}(\mathrm{~S}, \sigma)$. A product of two Weyl operators is defined by the canonical commutation relations in Weyl form,

$$
W(f) W(g)=\mathrm{e}^{-\mathrm{i} \sigma(f, g) / 2} W(f+g), \quad f, g \in \mathrm{S},
$$

and an involution by

$$
W(f)^{*}=W(-f), \quad f \in \mathrm{S},
$$

which may be extended by linearity and antilinearity, respectively, to $\mathfrak{A}_{0}(\mathrm{~S}, \sigma)$. Then $\mathfrak{A}_{0}(\mathrm{~S}, \sigma)$ becomes a unital ${ }^{*}$-algebra with unit $\mathbb{1}=W(0)$. To establish the existence of $W(f)$ satisfying (A.5) and (A.6) define $W(f)$ as the function $\mathrm{S} \longrightarrow \mathbb{C}$ with

$$
\mathrm{S} \ni g \mapsto W(f) g=\left\{\begin{array}{ll}
1 & \text { if } f=g \\
0 & \text { if } f \neq g
\end{array} .\right.
$$

The completion $\mathfrak{A}_{1}(\mathrm{~S}, \sigma)$ of $\mathfrak{A}_{0}(\mathrm{~S}, \sigma)$ with respect to the norm

$$
\left\|\sum_{k=1}^{n} z_{k} W\left(f_{k}\right)\right\|_{1}:=\sum_{k=1}^{n}\left|z_{k}\right|, \quad f_{i} \neq f_{j} \text { if } i \neq j,
$$

is a Banach *-algebra.

Now we introduce a $\mathrm{C}^{*}$-norm on $\mathfrak{A}_{0}(\mathrm{~S}, \sigma)$. Let $\mathscr{R}(\mathrm{S}, \sigma)$ be the set of all nondegenerate ${ }^{*}$-representations of the involutive Banach algebra $\mathfrak{A}_{1}(\mathrm{~S}, \sigma)$ on a Hilbert space. We introduce

$$
\|x\|=\sup \{\|\pi(x)\|: \pi \in \mathscr{R}(\mathrm{S}, \sigma)\}, \quad \text { for all } x \in \mathfrak{A}_{1}(\mathrm{~S}, \sigma) .
$$

Let us check that (A.8) is a $\mathrm{C}^{*}$-norm. If $x=\sum_{i=1}^{n} \lambda_{i} W\left(f_{i}\right)$ is some element in $\mathfrak{A}_{0}(\mathrm{~S}, \sigma)$ and if $\pi \in \mathscr{R}(\mathrm{S}, \sigma)$, then

$$
\|\pi(x)\| \leq \sum_{i=1}^{n}\left|\lambda_{i}\right| \cdot\left\|\pi\left(W\left(f_{i}\right)\right)\right\|=\|x\|_{1},
$$


thus $\|x\| \leq\|x\|_{1}$ for all $x \in \mathfrak{A}_{1}(\mathrm{~S}, \sigma)$. If $\|x\|=0$ then $\pi(x)=0$ for all $\pi \in \mathscr{R}(\mathrm{S}, \sigma)$, hence $x=0$. The triangle inequality for $\|\cdot\|$ is clear as well as homogeneity, moreover, we have $\left\|\pi\left(x^{*}\right)\right\|=\left\|\pi(x)^{*}\right\|$, hence $\left\|x^{*}\right\|=\|x\|$. The $\mathrm{C}^{*}$-property follows similarly, $\left\|\pi(x)^{*} \pi(x)\right\|=\|\pi(x)\|^{2}$ implies $\left\|x^{*} x\right\|=\|x\|^{2}$. Finally, we observe that $\|x y\| \leq\|x\| \cdot\|y\|$. Thus we have shown the following.

Proposition A.1 The completion $\mathfrak{A}(\mathrm{S}, \sigma)$ of $\mathfrak{A}_{0}(\mathrm{~S}, \sigma)$ with respect to the norm (A.8) is a $C^{*}$-algebra. This $C^{*}$-algebra is minimal in the following sense: Let $\|\cdot\|_{0}$ is another $C^{*}$-norm on $\mathfrak{A}_{1}(\mathrm{~S}, \sigma)$ then $\|x\|_{0} \leq\|x\|$ for all $x \in \mathfrak{A}_{0}(\mathrm{~S}, \sigma)$, i. e. $\tilde{\mathfrak{A}}_{1}(\mathrm{~S}, \sigma) \supseteq \mathfrak{A}(\mathrm{S}, \sigma)$, where $\tilde{\mathfrak{A}}_{1}(\mathrm{~S}, \sigma)$ denotes the completion with respect to $\|\cdot\|_{0}$.

Proof. It remains to show the last property. Let $\mathfrak{B}$ be the completion of $\mathfrak{A}_{1}(\mathrm{~S}, \sigma)$ with respect to $\|\cdot\|_{0}$. It has a faithful *-representation $\pi$ on $\mathscr{H}$; since $\mathfrak{B} \supseteq \mathfrak{A}_{1}(\mathrm{~S}, \sigma)$ it is also a *-representation of $\mathfrak{A}_{1}(\mathrm{~S}, \sigma)$. By faithfulness, $\|\pi(x)\|=\|x\|_{0}$ for all $x \in \mathfrak{B}$, hence we get from definition (A.8) $\|x\|_{0} \leq$ $\|x\|$ for all $x \in \mathfrak{A}_{1}(\mathrm{~S}, \sigma)$.

Definition A.2 Let $(\mathrm{S}, \sigma)$ be a symplectic group. The $C^{*}$-algebra $\mathfrak{A}(\mathrm{S}, \sigma)$ constructed in Proposition A.1 is called the CCR algebra of $(\mathrm{S}, \sigma)$.

Lemma A.3 Suppose that $(\mathrm{S}, \sigma)$ is a symplectic vector space with $\sigma$ nondegenerate. Then we have $\|W(f)-\mathbb{1}\|=2$ for all $f \in \mathrm{S} \backslash\{0\}$.

Proof. Clearly $W(f)$ is unitary in $\mathfrak{A}(\mathrm{S}, \sigma)$, and from (A.5) it follows that $W(g) W(f) W(g)^{*}=\mathrm{e}^{\mathrm{i} \sigma(f, g)} W(f)$ for all $f \in \mathrm{S}$, hence spec $W(f)$ is rotation invariant and thus equal to the unit circle. Then

$$
\begin{aligned}
\|W(f)-W(0)\| & =\mathrm{r}(W(f)-W(0)) \\
& =\sup \{|\lambda|: \lambda \in \operatorname{spec}(W(f)-W(0))\} \\
& =2,
\end{aligned}
$$

where $\mathrm{r}$ denotes the spectral radius.

\section{A.1.2. Properties}

The next lemma shows that the CCR algebra is simple provided $\sigma$ is nondegenerate, the proof we give is due to [101] with slight corrections.

Lemma A.4 Let $(\mathrm{S}, \sigma)$ be a symplectic group with $\sigma$ be nondegenerate. Then $\mathfrak{A}(\mathrm{S}, \sigma)$ is simple. 
Proof. Let $\pi$ be a nondegenerate ${ }^{*}$-representation of $\mathfrak{A}(\mathrm{S}, \sigma)$ on $\mathscr{H}$, we will show that $\pi$ is faithful. This implies simplicity since every closed left ideal is of the form ker $\pi$ for some $\pi \in \mathscr{R}(\mathrm{S}, \sigma)$. Assume without loss of generality that $\pi$ has a cyclic vector $\xi_{0}$. Observe that $\pi(W(0))$ is the identity $\mathbb{1}$ in $\mathrm{L}(\mathscr{H})$ since $\pi(W(0)) \pi(x) \xi_{0}=\pi(x) \xi_{0}$ for any $x \in \mathfrak{A}(\mathrm{S}, \sigma)$. Moreover, $\pi(W(f)) \neq 0$ since

$$
\pi(W(f)) \pi(W(f))^{*}=\pi(W(0))=\mathbb{1} .
$$

Next we show that $\sum_{i=1}^{n} \lambda_{i} W\left(f_{i}\right) \in \operatorname{ker} \pi$ for $\lambda_{1}, \ldots, \lambda_{n}$ implies that $\sum_{i=1}^{n} \lambda_{i} W\left(f_{i}\right)=0$. We proceed by induction on $n$. If $n=1$ the assertion is clear since $\pi(W(f)) \neq 0$. Assume that the assertion holds for $n>1$ and let $\sum_{i=1}^{n+1} \lambda_{i} W\left(f_{i}\right) \in \operatorname{ker} \pi$ and assume that $f_{i} \neq f_{j}$ if $i \neq j$. Then $\sum_{i=1}^{n+1} \lambda_{i} \pi\left(W\left(f_{i}\right)\right)=0$, and if we multiply this equality by $\pi\left(W\left(f_{n+1}\right)\right)^{*}$ from the right we obtain

$$
\begin{aligned}
0 & =\lambda_{n+1} \mathbb{1}+\sum_{i=1}^{n} \lambda_{i} \mathrm{e}^{\mathrm{i} \sigma\left(f_{i}, f_{n+1}\right) / 2} \pi\left(W\left(f_{i}-f_{n+1}\right)\right) \\
& =\lambda_{n+1} \mathbb{1}+\sum_{i=1}^{n} \mu_{i} \pi\left(W\left(g_{i}\right)\right),
\end{aligned}
$$

where $g_{i}=f_{i}-f_{n+1}$ and for $\mu_{i}=\mathrm{e}^{\mathrm{i} \sigma\left(f_{i}, f_{n+1}\right) / 2} \lambda_{i}=\mathrm{e}^{\mathrm{i} \sigma\left(g_{i}, f_{n+1}\right) / 2} \lambda_{i}, i=$ $1, \ldots, n$; hence $g_{i} \neq g_{j}$ if $i \neq j$ and $g_{i} \neq 0$ for $i=1, \ldots, n$. Multiplying this equality by $\pi(W(f))$ from the left and by $\pi\left(W(f)^{*}\right)$ from the right we obtain

$$
\begin{aligned}
0 & =\lambda_{n+1} \mathbb{1}+\sum_{i=1}^{n} \mu_{i} \pi\left(W\left(g_{i}\right)\right) \\
& =\lambda_{n+1} \mathbb{1}+\sum_{i=1}^{n} \mu_{i} \mathrm{e}^{-\mathrm{i} \sigma\left(f, g_{i}\right)} \pi\left(W\left(g_{i}\right)\right),
\end{aligned}
$$

for any $f \in \mathrm{S}$, and this leads to

$$
0=\sum_{i=1}^{n} \mu_{i}\left(1-\mathrm{e}^{-\mathrm{i} \sigma\left(f, g_{i}\right)}\right) \pi\left(W\left(g_{i}\right)\right)
$$

for any $f \in \mathrm{S}$. By the inductive hypothesis,

$$
0=\sum_{i=1}^{n} \mu_{i}\left(1-\mathrm{e}^{-\mathrm{i} \sigma\left(f, g_{i}\right)}\right) W\left(g_{i}\right)
$$


for any $f \in \mathrm{S}$ and since $W\left(g_{1}\right), \ldots, W\left(g_{n}\right)$ are linearly independent it follows that $\mu_{i}\left(1-\mathrm{e}^{-\mathrm{i} \sigma\left(f, g_{i}\right)}\right)=0$ for any $f \in \mathrm{S}$ and $i=1, \ldots, n$. But then $\mu_{1}=\cdots=\mu_{n}=0$ since $\sigma$ is nondegenerate, consequently $\lambda_{1}=\cdots=$ $\lambda_{n}=0$, and we are done. Thus the restriction of $\pi$ to $\mathfrak{A}_{0}(\mathrm{~S}, \sigma)$ is faithful, and we can also conclude that the extension to $\mathfrak{A}(\mathrm{S}, \sigma)$ is faithful.

A Weyl system is a map $w: \mathbf{S} \longrightarrow \mathscr{U}(\mathscr{H})$ of $\mathbf{S}$ into the unitary group on the Hilbert space $\mathscr{H}$ such that

$$
w(f) w(g)=\mathrm{e}^{-\mathrm{i} \sigma(f, g) / 2} w(f+g) \text { for all } f, g \in \mathrm{S},
$$

and such that $[\{w(f) \xi: f \in \mathrm{S}, \xi \in \mathscr{H}\}]=\mathscr{H}$.

Theorem A.5 Every Weyl system $\{w(f): f \in \mathrm{S}\}$ on $(\mathrm{S}, \sigma)$ induces a unique representation $\pi$ of $\mathfrak{A}(\mathrm{S}, \sigma)$ such that $\pi(W(f))=w(f)$ for any $f \in \mathrm{S}$. Conversely, if $\pi \in \mathscr{R}(\mathrm{S}, \sigma)$ then $f \mapsto \pi(W(f))$ is a Weyl system.

Proof. Define $\pi: \mathfrak{A}_{0}(\mathrm{~S}, \sigma) \longrightarrow \mathrm{L}(\mathscr{H})$ by $\pi(W(f))=w(f)$ and extension by linearity. Then we see as in (A.9) that $\|\pi(x)\| \leq\|x\|$ for any $x \in$ $\mathfrak{A}_{0}(\mathrm{~S}, \sigma)$, thus $\pi$ extends to $\mathfrak{A}_{1}(\mathrm{~S}, \sigma)$ and is in $\mathscr{R}(\mathrm{S}, \sigma)$ as a consequence of $[\{w(f) \xi: f \in \mathrm{S}, \xi \in \mathscr{H}\}]=\mathscr{H}$. Then clearly $\|\pi(x)\| \leq\|x\|$, so $\pi$ extends to $\mathfrak{A}(\mathrm{S}, \sigma)$. Now using (A.10) it is easy to see that $\pi$ is a ${ }^{*}$-homomorphism and thus a ${ }^{*}$-representation on $\mathscr{H}$. The converse is trivial.

Corollary A.6 Let $\sigma$ be nondegenerate and $f \mapsto w(f) \in \mathscr{U}(\mathrm{H})$ a Weyl system. Let $\mathfrak{A}=\varlimsup \operatorname{lin}\{w(f): f \in \mathrm{S}\}$. Then $\mathfrak{A}$ is ${ }^{*}$-isomorphic to $\mathfrak{A}(\mathrm{S}, \sigma)$.

Proof. Let $\pi$ be the representation constructed in Theorem A.5 with the property $\pi(W(f))=w(f)$. Since $\mathfrak{A}(\mathrm{S}, \sigma)$ is simple, $\pi$ is faithful, and $\pi(\mathfrak{A}(\mathrm{S}, \sigma))$ is a $\mathrm{C}^{*}$-subalgebra of $\mathfrak{A}$ and, in particular, closed. Now by the isomorphism theorem $\mathfrak{A}(\mathrm{S}, \sigma) / \operatorname{ker} \pi \cong \pi(\mathfrak{A}(\mathrm{S}, \sigma))$, hence $\pi$ must also be surjective.

The following lemma will be used in the next section.

Lemma A.7 Let $\psi: \mathfrak{A}_{0}(\mathrm{~S}, \sigma) \longrightarrow \mathbb{C}$ be a positive linear functional. Then $\psi$ extends to a positive linear functional on $\mathfrak{A}(\mathrm{S}, \sigma)$.

Proof. There exists a state $\omega$ on $\mathfrak{A}(\mathrm{S}, \sigma)$ such that $\psi \leq \lambda \omega$, where $\lambda \geq 0$. Since $|\omega(W(f))| \leq \omega(W(0))=1$ by the Cauchy-Schwarz inequality, it follows that

$$
\left|\omega\left(\sum_{i=1}^{n} \lambda_{i} W\left(f_{i}\right)\right)\right| \leq \sum_{i=1}^{n}\left|\lambda_{i}\right|=\|x\|_{1},
$$


thus $\omega$ extends to a state on $\mathfrak{A}_{1}(\mathrm{~S}, \sigma)$. If $\left(\pi_{\omega}, \mathscr{H}_{\omega}, \xi_{\omega}\right)$ is the associated GNS triplet we can choose $\omega$ such that $\pi_{\omega} \in \mathscr{R}(\mathrm{S}, \sigma)$. Then

$$
|\psi(x)|^{2} \leq \lambda^{2}|\omega(x)|^{2} \leq \lambda^{2}\left\|\pi_{\omega}(x) \xi_{\omega}\right\| \leq \lambda^{2}\left\|\pi_{\omega}(x)\right\|,
$$

and hence $|\psi(x)| \leq \lambda\|x\|$ for all $x \in \mathfrak{A}_{1}(\mathrm{~S}, \sigma)$, by the definition (A.8), and we conclude.

\section{A.1.3. Generating Functionals}

A useful tool to study representations of the CCR algebra (or equivalently of the canonical commutation relations, cf. Theorem A.5) is the concept of the generating functional, introduced in [37].

Definition A.8 Let $(\mathrm{S}, \sigma)$ be a symplectic group. Then $\phi: \mathrm{S} \longrightarrow \mathbb{C}$ is called a generating functional provided it satisfies the following properties:

1. $\phi(0)=1$.

2. For all $n \in \mathbb{N}, f_{1}, \ldots, f_{n} \in \mathrm{S}$ and $\lambda_{1}, \ldots, \lambda_{n} \in \mathbb{C}$ we have

$$
\sum_{i, j=1}^{n} \bar{\lambda}_{i} \lambda_{j} \mathrm{e}^{\mathrm{i} \sigma\left(f_{i}, f_{j}\right) / 2} \phi\left(f_{i}-f_{j}\right) \geq 0 .
$$

The following result explains the name "generating functional".

Proposition A.9 Let $\omega$ be a state on $\mathfrak{A}(\mathrm{S}, \sigma)$. Then $f \mapsto \omega(W(f))$ is a generating functional. Conversely, if $\phi$ is a generating functional there exists a unique state $\omega_{\phi}$ on $\mathfrak{A}(\mathrm{S}, \sigma)$ such that $\phi(f)=\omega_{\phi}(W(f))$ for all $f \in \mathrm{S}$.

Proof. Let $\omega$ be a state and let $x=\sum_{i=1}^{n} \lambda_{i} W\left(f_{i}\right) \in \mathfrak{A}_{0}(\mathrm{~S}, \sigma)$. Then

$$
\begin{aligned}
0 & \leq \omega\left(x^{*} x\right) \\
& =\sum_{i, j=1}^{n} \bar{\lambda}_{i} \lambda_{j} \omega\left(W\left(f_{i}\right)^{*} W\left(f_{j}\right)\right) \\
& =\sum_{i, j=1}^{n} \bar{\lambda}_{i} \lambda_{j} \mathrm{e}^{\mathrm{i} \sigma\left(f_{i}, f_{j}\right) / 2} \omega\left(W\left(f_{j}-f_{i}\right)\right),
\end{aligned}
$$

so $f \mapsto \omega(W(f))$ is a generating functional. Conversely, let $\phi$ be a generating functional and define $\omega_{\phi}: \mathfrak{A}_{0}(\mathrm{~S}, \sigma) \longrightarrow \mathbb{C}$ by

$$
\omega_{\phi}(x)=\sum_{i=1}^{n} \lambda_{i} \phi\left(f_{i}\right), \quad \text { where } x=\sum_{i=1}^{n} \lambda_{i} W\left(f_{i}\right) \in \mathfrak{A}_{0}(\mathrm{~S}, \sigma) .
$$


In view of (A.11) this defines a positive functional on $\mathfrak{A}_{0}(\mathrm{~S}, \sigma)$ and since $\omega_{\phi}(\mathbb{1})=1$ it is a state. By Lemma A.7 it extends to a state on $\mathfrak{A}(\mathrm{S}, \sigma)$ with the required property.

If $\phi$ is a generating functional and $\omega_{\phi}$ the corresponding state we shall denote the GNS triplet associated with $\omega_{\phi}$ by $\left(\pi_{\phi}, \mathscr{H}_{\phi}, \xi_{\phi}\right)$.

\section{A.1.4. Automorphisms}

Proposition A.10 Suppose $S: \mathrm{S} \longrightarrow \mathrm{S}$ is a surjective linear map such that $\sigma(S(f), S(g))=\sigma(f, g)$ or $\sigma(S(f), S(g))=-\sigma(f, g)$ for all $f, g \in \mathrm{S}$. Then there exists a unique ${ }^{*}$-automorphism or ${ }^{*}$-anti-automorphism $\alpha_{S}$ on $\mathfrak{A}(\mathrm{S}, \sigma)$ such that $\alpha_{S}(W(f))=W(S(f))$ for all $f \in \mathrm{S}$.

Proof. Define $\alpha_{S}$ on $\mathfrak{A}_{0}(\mathrm{~S}, \sigma)$ by $\alpha_{S}(W(f))=W(S(f))$ and extension by linearity; then $\alpha_{S}$ is a $*$-automorphism or *-anti-automorphism with $\left(\alpha_{S}\right)^{-1}=\alpha_{S^{-1}}$. Clearly, $\left\|\alpha_{S}(x)\right\|_{1}=\|x\|_{1}$ for all $x \in \mathfrak{A}_{0}(\mathrm{~S}, \sigma)$ and $\alpha_{S}$ extends to $\mathfrak{A}_{1}(\mathrm{~S}, \sigma)$, by surjectivity of $S$ this extension is a ${ }^{*}$-automorphism or *-anti-automorphism. Let $\pi \in \mathscr{R}(\mathrm{S}, \sigma)$, then $\pi \circ \alpha_{S} \in \mathscr{R}(\mathrm{S}, \sigma)$, thus $\left\|\alpha_{S}(x)\right\|=\sup _{\pi \in \mathscr{R}(\mathrm{S}, \sigma)}\left\|\left(\pi \circ \alpha_{S}\right)(x)\right\| \leq \sup _{\pi \in \mathscr{R}(\mathrm{S}, \sigma)}\|\pi(x)\|=\|x\|$ for all $x \in \mathfrak{A}_{1}(\mathrm{~S}, \sigma)$. Thus $\alpha_{S}$ extends to $\mathfrak{A}(\mathrm{S}, \sigma)$ and again $\alpha_{S}$ is a *automorphism or *-anti-automorphism.

A linear map $S: \mathrm{S} \longrightarrow \mathrm{S}$ such that $\sigma(S(f), S(g))=\sigma(f, g)$ for all $f, g \in \mathrm{S}$ is called symplectic.

We now show that if a symplectic group leaves invariant a generating functional then the corresponding automorphism group is unitarily implemented in the representation belonging to the generating functional.

Proposition A.11 Let $\left\{S_{t}\right\}_{t \in \mathbb{R}}$ be a group of symplectic maps and $\phi$ a generating functional on $(\mathrm{S}, \sigma)$. Suppose that $\mathbb{R} \ni t \mapsto \phi\left(S_{t}(f)+g\right)$ and $\mathbb{R} \ni t \mapsto \sigma\left(f, S_{t}(g)\right)$ are continuous for all $f, g \in \mathrm{S}$, and that $\phi\left(S_{t}(f)\right)=$ $\phi(f)$ for all $t \in \mathbb{R}$ and $f \in \mathrm{S}$. Then there exists a strongly continuous unitary group $\left\{u_{t}\right\}_{t \in \mathbb{R}} \subseteq \mathscr{U}\left(\mathscr{H}_{\phi}\right)$ such that

$$
\begin{aligned}
u_{t} \pi_{\phi}(x) \xi_{\phi} & =\pi_{\phi}\left(\alpha_{t}(x)\right) \xi_{\phi}, \\
\pi_{\phi}\left(\alpha_{t}(x)\right) & =u_{t} \pi_{\phi}(x) u_{t}^{*},
\end{aligned}
$$

for all $t \in \mathbb{R}$ and $x \in \mathfrak{A}(\mathrm{S}, \sigma)$. Here $\alpha_{t} \in$ Aut $\mathfrak{A}(\mathrm{S}, \sigma)$ is the automorphism such that $\alpha_{t}(W(f))=W\left(S_{t}(f)\right)$ (see Proposition A.10). 
Proof. For $x=\sum_{i=1}^{n} \lambda_{i} W\left(f_{i}\right) \in \mathfrak{A}_{0}(\mathrm{~S}, \sigma)$ and $t \in \mathbb{R}$ we have

$$
\omega_{\phi}\left(\alpha_{t}(x)\right)=\sum_{i=1}^{n} \lambda_{i} \omega_{\phi}\left(W\left(S_{t}(f)\right)\right)=\sum_{i=1}^{n} \lambda_{i} \phi\left(S_{t}(f)\right)=\omega_{\phi}(x),
$$

and if we define $u_{t}$ on $\pi_{\phi}\left(\mathfrak{A}_{0}(\mathrm{~S}, \sigma)\right) \xi_{\phi}$ by (A.12) then

$$
\begin{aligned}
\left\langle u_{t} \pi_{\phi}(y) \xi_{\phi}, u_{t} \pi_{\phi}(x) \xi_{\phi}\right\rangle & =\left\langle\pi_{\phi}\left(\alpha_{t}(y)\right) \xi_{\phi}, \pi_{\phi}\left(\alpha_{t}(x)\right) \xi_{\phi}\right\rangle \\
& =\left\langle\xi_{\phi}, \pi_{\phi}\left(\alpha_{t}\left(y^{*} x\right)\right) \xi_{\phi}\right\rangle \\
& =\left\langle\xi_{\phi}, \pi_{\phi}\left(y^{*} x\right) \xi_{\phi}\right\rangle \\
& =\left\langle\pi_{\phi}(y) \xi_{\phi}, \pi_{\phi}(x) \xi_{\phi}\right\rangle
\end{aligned}
$$

for any $x, y \in \mathfrak{A}_{0}(\mathrm{~S}, \sigma)$, so $u_{t}$ extends to a unitary operator on $\mathscr{H}_{\phi}$. We next check (A.13), to this end let $x, y, z \in \mathfrak{A}(\mathrm{S}, \sigma)$. Then

$$
\begin{aligned}
\left\langle\pi_{\phi}(x) \xi_{\phi}, u_{t} \pi_{\phi}(x) u_{t}^{*} \pi_{\phi}(z) \xi_{\phi}\right\rangle & =\left\langle\xi_{\phi}, \pi_{\phi}\left(\alpha_{-t}\left(x^{*}\right) y \alpha_{-t}(z)\right) \xi_{\phi}\right\rangle \\
& =\omega_{\phi}\left(\alpha_{-t}\left(x^{*}\right) y \alpha_{-t}(z)\right) \\
& =\omega_{\phi}\left(x^{*} \alpha_{t}(y) z\right) \\
& =\left\langle\pi_{\phi}(x) \xi_{\phi}, \pi_{\phi}\left(\alpha_{t}(y)\right) \pi_{\phi}(z) \xi_{\phi}\right\rangle
\end{aligned}
$$

and the claim follows since $\xi_{\phi}$ is cyclic. Now let $x, y \in \mathfrak{A}_{0}(\mathrm{~S}, \sigma)$ with $x=\sum_{i=1}^{n} \lambda_{i} W\left(f_{i}\right), y=\sum_{j=1}^{m} \mu_{j} W\left(g_{j}\right)$. Then the map

$$
\begin{aligned}
t \mapsto\left\langle\pi_{\phi}(x) \xi_{\phi}, u_{t} \pi_{\phi}(y)\right. & \left.\xi_{\phi}\right\rangle=\omega_{\phi}\left(x^{*} \alpha_{t}(y)\right) \\
= & \sum_{i=1}^{n} \sum_{j=1}^{m} \bar{\lambda}_{i} \mu_{j} \omega_{\phi}\left(W\left(-f_{j}\right) W\left(S_{t}\left(g_{i}\right)\right)\right) \\
& =\sum_{i=1}^{n} \sum_{j=1}^{m} \bar{\lambda}_{i} \mu_{j} \omega_{\phi}\left(W\left(S_{t}\left(g_{j}\right)-f_{i}\right)\right) \mathrm{e}^{-\mathrm{i} \sigma\left(g_{j}, S_{t}\left(f_{i}\right)\right) / 2} \\
& =\sum_{i=1}^{n} \sum_{j=1}^{m} \bar{\lambda}_{i} \mu_{j} \phi\left(S_{t}\left(g_{j}\right)-f_{i}\right) \mathrm{e}^{-\mathrm{i} \sigma\left(g_{j}, S_{t}\left(f_{i}\right)\right) / 2}
\end{aligned}
$$

is continuous. Since $\pi_{\phi}\left(\mathfrak{A}_{0}(\mathrm{~S}, \sigma)\right) \xi_{\phi}$ is norm-dense in $\mathscr{H}_{\phi}$ it follows that $\left\{u_{t}\right\}_{t \in \mathbb{R}}$ is weakly and hence strongly continuous.

\section{A.2. Regular and Quasifree Representations}

In the following we shall assume that $(\mathrm{S}, \sigma)$ is a symplectic space. 


\section{A.2.1. Regular Representations}

Definition A.12 $A{ }^{*}$-representation $\pi$ of the $C C R$ algebra $\mathfrak{A}(\mathrm{S}, \sigma)$ on a Hilbert space $\mathscr{H}$ is called regular if the map $t \mapsto \pi(W(t f))$ is continuous in the weak operator topology for any $f \in \mathrm{S}$. A state $\omega$ is called regular if the corresponding GNS representation $\left(\pi_{\omega}, \mathscr{H}_{\omega}, \xi_{\omega}\right)$ is regular.

Since $t \mapsto \pi(W(t f))=u_{t}$ is a unitary group acting on $\mathscr{H}$ it follows that if $\pi$ is regular the map $t \mapsto \pi(W(t f))$ is continuous is the strong operator topology.

Proposition A.13 Let $\phi$ be a generating functional. Then the corresponding representation $\pi_{\phi}$ is regular if and only if the map $t \mapsto \phi(t f+g)$ is continuous for all $f, g \in \mathrm{S}$.

Proof. Assume that $\pi_{\phi}$ is regular, then for any $f, g \in \mathrm{S}$ the map

$$
\begin{aligned}
t \mapsto \phi(t f+g) & =\omega_{\phi}(W(t f+g))=\left\langle\xi_{\phi}, \pi_{\phi}(W(t f+g)) \xi_{\phi}\right\rangle \\
& =\mathrm{e}^{-\mathrm{i} t \sigma(f, g)}\left\langle\xi_{\phi}, \pi_{\phi}(W(t f)) \pi(W(g)) \xi_{\phi}\right\rangle
\end{aligned}
$$

is continuous. Conversely, assume that $t \mapsto \phi(t f+g)$ is continuous. Choose $\xi \in \mathscr{H}$ and $\epsilon>0$, then there is $x \in \mathfrak{A}_{0}(\mathrm{~S}, \sigma)$ such that $\left\|\pi_{\phi}(x) \xi_{\phi}-\xi\right\|<\epsilon$. It follows that

$$
\begin{aligned}
& \left\|\pi_{\phi}(W(t f)) \xi-\xi\right\| \\
& \quad \leq\left\|\pi_{\phi}(W(t f)) \xi-\pi_{\phi}(W(t f)) \pi_{\phi}(x) \xi_{\phi}\right\| \\
& \quad \quad+\left\|\pi_{\phi}(W(t f)) \pi_{\phi}(x) \xi_{\phi}-\pi_{\phi}(x) \xi_{\phi}\right\|+\left\|\pi_{\phi}(x) \xi_{\phi}-\xi\right\| \\
& \leq 2\left\|\pi_{\phi}(x) \xi_{\phi}-\xi\right\|+\left\|\pi_{\phi}(W(t f)) \pi_{\phi}(x) \xi_{\phi}-\pi_{\phi}(x) \xi_{\phi}\right\| \\
& \leq 2 \epsilon+\left\|\pi_{\phi}(W(t f) x-x) \xi_{\phi}\right\| \\
& =2 \epsilon+\omega_{\phi}\left[(W(t f) x-x)^{*}(W(t f) x-x)\right]^{1 / 2} \\
& =2 \epsilon+\left[2 \omega_{\phi}\left(x^{*} x\right)-2 \operatorname{Re} \omega_{\phi}\left(x^{*} W(t f) x\right)\right]^{1 / 2} \\
& =2 \epsilon+\left[2 \omega_{\phi}\left(x^{*} x\right)\right. \\
& \left.\quad-2 \operatorname{Re} \sum_{i, j=1}^{n} \bar{\lambda}_{i} \lambda_{j} \mathrm{e}^{\mathrm{i} \sigma\left(f_{i}, t f\right) / 2} \mathrm{e}^{\mathrm{i} \sigma\left(f_{t}+t f, f_{j}\right)} \phi\left(t f+f_{j}-f_{i}\right)\right]^{1 / 2},
\end{aligned}
$$

if $x=\sum_{i=1}^{n} \lambda_{i} W\left(f_{i}\right)$. By hypothesis we see that the second term on the right hand side becomes as small as we please if $t \rightarrow 0$.

Let $\omega$ be a regular state on $\mathfrak{A}(\mathrm{S}, \sigma)$ and let $\Phi_{\omega}(f)$ be the generator of the unitary group $\left\{\pi_{\omega}(W(t f))\right\}_{t \in \mathbb{R}}$, where $f \in \mathrm{S}$. We call $\Phi_{\omega}(f)$ the 
field operator evaluated at the test function $f$; it is a self-adjoint operator on $\mathscr{H}_{\omega}$. Moreover, we can show (see [7]) the following: For every regular state $\omega$ the set of operators $\left\{\Phi_{\omega}(f), \Phi_{\omega}(\right.$ if $\left.f): f \in M\right\}$ has a common dense set of analytic vectors, where $M \subseteq \mathrm{S}$ is any finite-dimensional subspace. It is easy to check that $\Phi_{\omega}(f)$ is linear in $f$. We define the creation and annihilation operators by

$$
\begin{aligned}
\operatorname{dom} a_{\omega}(f)= & \operatorname{dom} a_{\omega}^{*}(f)=\operatorname{dom} \Phi_{\omega}(f) \cap \operatorname{dom} \Phi_{\omega}(\mathrm{i} f), \\
& a_{\omega}(f)=2^{-1 / 2}\left(\Phi_{\omega}(f)+\mathrm{i} \Phi_{\omega}(\mathrm{i} f)\right), \\
& a_{\omega}^{*}(f)=2^{-1 / 2}\left(\Phi_{\omega}(f)-\mathrm{i} \Phi_{\omega}(\mathrm{i} f)\right) .
\end{aligned}
$$

One can show that they are densely defined and closed, and that $a_{\omega}^{*}(f)$ is the adjoint of $a_{\omega}(f)$ for any $f \in \mathrm{S}$.

Proposition A.14 Let $\omega$ be a regular state on $\mathfrak{A}(\mathrm{S}, \sigma)$ and suppose that $\xi_{\omega} \in \operatorname{dom} \Phi_{\omega}(f)$ for all $f \in \mathrm{S}$. Then

$$
D=\operatorname{lin}\left\{\pi_{\omega}(W(g)) \xi_{\omega}: g \in \mathrm{S}\right\} \subseteq \operatorname{dom} \Phi_{\omega}(f) \quad \text { for all } f \in \mathrm{S},
$$

and $D$ is a common core for all $\Phi_{\omega}(f), f \in \mathrm{S}$. Moreover, we have

$$
\left[\Phi_{\omega}(f), \pi_{\omega}(W(g))\right] \xi=-\sigma(f, g) \pi_{\omega}(W(g)) \xi, \quad f, g \in \mathrm{S}, \xi \in D .
$$

For $f, g \in \mathrm{S}$ we have $\Phi_{\omega}(f) D \subseteq \operatorname{dom} \Phi_{\omega}(g)$, and

$$
\left[\Phi_{\omega}(f), \Phi_{\omega}(g)\right] \xi=\mathrm{i} \sigma(f, g) \xi
$$

for all $\xi \in D$.

Proof. Write $\pi_{\omega}(f)=W_{\omega}(f)$. For $f, g \in \mathrm{S}$, consider the following identity which follows from (A.5), and whose right hand side is strongly differentiable in $t$,

$$
W_{\omega}(t f) W_{\omega}(g) \xi_{\omega}=\mathrm{e}^{-\mathrm{i} t \sigma(f, g)} W_{\omega}(g) W_{\omega}(t f) \xi_{\omega} .
$$

The derivative of this equation at $t=0$ is given by

$$
\Phi_{\omega}(f) W_{\omega}(g) \xi_{\omega}=-\sigma(f, g) W_{\omega}(g) \xi_{\omega}+W_{\omega}(g) \Phi_{\omega}(f) \xi_{\omega},
$$

thus we obtain $W_{\omega}(g) \xi_{\omega} \in \operatorname{dom} \Phi_{\omega}(f)$, hence $D \subseteq \operatorname{dom} \Phi_{\omega}(f)$. Using this result we get by a similar calculation that

$$
\left[\phi_{\omega}(f), W_{\omega}(g)\right] \xi=-\sigma(f, g) W_{\omega}(g) \xi .
$$


By the Weyl relations, it follows that $W_{\omega}(t f) D=\mathrm{e}^{\mathrm{i} t \Phi_{\omega}(f)} \subseteq D$ for any $t \in \mathbb{R}$, hence $D$ is a core for $\Phi_{\omega}(f)$. To prove the last statement take $\xi=W_{\omega}(h) \in D, h \in \mathrm{S}$. Then for $s, t \in \mathbb{R}$,

$$
W_{\omega}(t f) W_{\omega}(s g) \xi=\mathrm{e}^{-\mathrm{i} s t \sigma(f, g)} W_{\omega}(s g) W_{\omega}(t f) \xi,
$$

differentiability shows that $\Phi_{\omega}(f) D \subseteq \operatorname{dom} \Phi_{\omega}(g)$, and differentiation with respect to $s$ and $t$ at 0 yields the desired result.

A state $\omega$ on $\mathfrak{A}(\mathrm{S}, \sigma)$ is said to be of class $C^{m}$ if the map $t \mapsto \omega(W(t f))$ is $m$-times differentiable at $t=0$ for any $f \in \mathrm{S}$. A vector $\xi \in \mathscr{H}_{\omega}$ is said to be of class $C^{m}$ if $t \mapsto \pi_{\omega}(W(t f)) \xi$ is $m$-times strongly differentiable. This condition is equivalent to $\xi \in \operatorname{dom} \Phi_{\omega}(f)$. Moreover, it can be checked that $\omega$ is $C^{2 m}$ if and only if $\xi_{\omega}$ is of class $C^{m}$. If $\omega$ is of class $C^{\infty}$ then arbitrary polynomials of $\Phi_{\omega}(f)$ can be defined; in particular, we can define the $n$-point functions

$$
\begin{aligned}
\omega\left(\Phi_{\omega}\left(f_{1}\right) \cdots \Phi_{\omega}\left(f_{n}\right)\right) & =\left\langle\xi_{\omega}, \Phi_{\omega}\left(f_{1}\right) \cdots \Phi_{\omega}\left(f_{n}\right) \xi_{\omega}\right\rangle, \\
\omega\left(x \Phi_{\omega}\left(f_{1}\right) \cdots \Phi_{\omega}\left(f_{n}\right)\right) & =\left\langle\xi_{\omega}, \pi_{\omega}(x) \Phi_{\omega}\left(f_{1}\right) \cdots \Phi_{\omega}\left(f_{n}\right) \xi_{\omega}\right\rangle,
\end{aligned}
$$

where $f_{1}, \ldots, f_{n} \in \mathrm{S}$ and $x \in \mathfrak{A}(\mathrm{S})$. Finally, we remark that for a regular representation we can introduce field operators as well as creation and annihilation operators in a similar manner.

A state $\omega$ is called (entire) analytic if the function $t \mapsto \omega(W(t f))$ is (entire) analytic for any $f \in \mathrm{S}$ in an open interval around 0 . It can be shown that $\omega$ is analytic if and only if $\xi_{\omega}$ is an analytic vector for all $\Phi_{\omega}(f)$, $f \in \mathrm{S}$. Secondly, if $\omega$ is analytic then $t \mapsto \omega(W(t f))$ is analytic in an open strip around the real axis (see [7]). By Proposition A.9 the state $\omega$ is determined by its values on the Weyl operators, but if $\omega$ is analytic it is determined by the derivatives at 0 of $t \mapsto \omega(W(t f))=\phi(t f)$, i. e. by the set $\left\{\omega\left(\Phi_{\omega}(f)\right): f \in \mathbf{S}, n \in \mathbb{N}\right\}$, or equivalently by $\left\{\omega\left(a_{\omega}^{*}(f)^{n} a_{\omega}(f)\right)^{n}\right.$ : $f \in \mathrm{S}, n \in \mathbb{N}\}$. Thus in this case the state is determined by its $n$-point functions.

For regular representations of systems with finitely many degrees of freedom we have the familiar Stone-von Neumann uniqueness theorem, which we state now. Before we define Fock representations.

Definition A.15 Let $\pi$ be a regular representation of $\mathfrak{A}(\mathrm{S}, \sigma)$ on $\mathscr{H}$. Then $\pi$ is called a Fock representation if there exists a nonzero vector $\xi_{0} \in \mathscr{H}$ such that $a_{\pi}(f) \xi_{0}=0$ for any $f \in \mathrm{S}$. Here $a_{\pi}$ is the annihilation operator associated to the representation $\pi$.

The normalized vector $\xi_{0}$ of a Fock representation is called the Fock vacuum vector. The next is the Stone-von Neumann theorem. 
Theorem A.16 Let $(\mathrm{S}, \sigma)$ be a finite-dimensional symplectic space with $\sigma$ nondegerenate. Then all regular representations of the CCR algebra are unitarily equivalent up to multiplicity. More precisely, in this case any representation is unitarily equivalent up to multiplicity to a Fock representation.

In the following we shall work with a symplectic space $(\mathrm{S}, \sigma)$ with a nondegenerate symplectic form $\sigma$.

From the previous theorem all irreducible Fock representations are unitarily equivalent, hence we can speak of the Fock representation. In this context another notion of equivalence of representations is useful. Let $\pi$ be a representation of a $\mathrm{C}^{*}$-algebra $\mathfrak{A}$. Then a state $\psi \in \mathscr{S}(\mathfrak{A})$ is called $\pi$-normal provided there exists a normal state $\varphi$ on the von Neumann algebra $\pi(\mathfrak{A})^{\prime \prime}$ such that $\psi(x)=\varphi(\pi(x))$ for all $x \in \mathfrak{A}$. The set of all $\pi$-normal states is denoted by $N_{\pi}$. If $\omega$ is a state on $\mathfrak{A}$ then $N_{\omega}$ denotes the set of all states in $\mathscr{S}(\mathfrak{A})$ which are $\pi_{\omega}$-normal. The set $N_{\omega}$ or $N_{\pi} \subseteq \mathscr{S}(\mathfrak{A})$ is a folium; it is sometimes called the folium of the state or the representation. Two representations $\pi_{1}$ and $\pi_{2}$ are called quasiequivalent if $N_{\pi_{1}}=N_{\pi_{2}}$; similarly, two states $\omega_{1}, \omega_{2} \in \mathscr{S}(\mathfrak{A})$ are called quasi-equivalent if $N_{\omega_{1}}=N_{\omega_{2}}$. Now by Theorem 2.4.26. of [6] we have the following:

Theorem A.17 Let $\pi_{1}$ and $\pi_{2}$ be two (nondegenerate, i. e. $\left[\pi_{i}(\mathfrak{A}) \mathscr{H}_{i}\right]=$ $\left.\mathscr{H}_{i}\right)$ representations of a $C^{*}$-algebra $\mathfrak{A}$. Then the following assertions are equivalent:

1. $\pi_{1}$ and $\pi_{2}$ are quasi-equivalent.

2. There exists a cardinal number $n$ such that $n \pi_{1}$ and $n \pi_{2}$ are unitarily equivalent (i. e. $\pi_{1}$ and $\pi_{2}$ are unitarily equivalent up to multiplicity).

3. There exists an automorphism $\alpha: \pi_{1}(\mathfrak{A})^{\prime \prime} \longrightarrow \pi_{2}(\mathfrak{A})^{\prime \prime}$ such that $\alpha\left(\pi_{1}(x)\right)=\pi_{2}(x)$ for all $x \in \mathfrak{A}$.

Thus we can express the Stone-von Neumann theorem as follows: If $(\mathrm{S}, \sigma)$ is finite-dimensional then any regular representation of $\mathfrak{A}(\mathrm{S}, \sigma)$ is quasiequivalent to the Fock representation.

The Fock representation is characterized by an important property, namely by the existence of a number operator [7]. Let $\mathfrak{A}(\mathrm{S}, \sigma)$ be the CCR algebra over a Hilbert space $\mathbf{S}$ with canonical symplectic form $\sigma(f, g)=$ $\operatorname{Im}\langle f, g\rangle$, and let $\omega$ be a regular state on $\mathfrak{A}(\mathrm{S}, \sigma)$. Consider a finitedimensional symplectic subspace $F \subseteq \mathrm{S}$ and define a quadratic form $n_{\omega, F}$ 
by

$$
\begin{aligned}
\operatorname{dom} n_{\omega, F} & =\bigcap_{f \in F} \operatorname{dom} a_{\omega}(f), \\
n_{\omega, F}(\xi) & =\sum_{i}\left\|a_{\omega}\left(f_{i}\right)\right\|^{2},
\end{aligned}
$$

where $\left\{f_{i}\right\}$ is an orthonormal basis of $F$. It is not difficult to see that $n_{\omega, F}$ does not depend on the choice of this basis. Then $\left\{n_{\omega, F}\right\}_{F}$ is a net of quadratic forms (the finite-dimensional subspaces $F$ are ordered by inclusion). Now define a quadratic form $n_{\omega}$ by

$$
\begin{aligned}
\operatorname{dom} n_{\omega} & =\left\{\xi \in \bigcap_{F} \operatorname{dom} n_{\omega, F}: \sup _{F} n_{\omega, F}(\xi)<\infty\right\}, \\
n_{\omega}(\xi) & =\sup _{F} n_{\omega, F}(\xi) .
\end{aligned}
$$

Suppose that $\operatorname{dom} n_{\omega} \subseteq \mathrm{S}$ is dense and let $N_{\omega, F}$ and $N_{\omega}$ denote the self-adjoint operators on $\mathscr{H}_{\omega}$ corresponding to the quadratic forms $n_{\omega, F}$ and $n_{\omega}$. One can show (Lemma 5.2.13. of [7]) that then

$$
\lim _{F}\left\|\mathrm{e}^{\mathrm{i} t N_{\omega, F}} \xi-\mathrm{e}^{\mathrm{i} t N_{\omega}} \xi\right\|=0
$$

for any $\xi \in \mathscr{H}_{\omega}$ and uniformly for $t$ in compact real intervals. Now we have the following.

Theorem A.18 Let $(\mathrm{S}, \sigma)$ be a complex Hilbert space and let $\omega$ be a regular state on $\mathfrak{A}(\mathrm{S}, \sigma)$. Then the following assertions are equivalent:

1. The state $\omega$ lies in the folium of the Fock representation.

2. $\pi_{\omega}$ is quasi-equivalent to the Fock representation.

3. $\operatorname{dom} n_{\omega}$ is dense in $\mathrm{S}$, i. e. the so-called number operator $N_{\omega}$ exists.

4. $\operatorname{dom} n_{\omega}$ contains a vector which is cyclic for $\pi_{\omega}(\mathfrak{A}(\mathrm{S}, \sigma))$.

For a proof see [7]. Formally, the number operator can be written as

$$
N_{\omega}=\sum_{k=1}^{\infty} a_{\omega}^{*}\left(f_{k}\right) a_{\omega}\left(f_{k}\right),
$$

where $\left\{f_{k}\right\}_{k \in \mathbb{N}}$ is an orthonormal basis of S. 


\section{A.2.2. Quasifree States and Representations}

We now turn to a particularly important subclass of states on the CCR algebra, namely to the quasifree states. They were introduced in [113] and studied in the algebraic framework in [102]. Let $(\mathrm{S}, \sigma)$ be a symplectic space with a not necessarily nondegenerate symplectic form $\sigma$ and let $\mathscr{A}$ be the set of all real symmetric positive bilinear forms $s: \mathrm{S} \times \mathrm{S} \longrightarrow \mathbb{R}$ such that

$$
|\sigma(f, g)|^{2} \leq s(f, f) s(g, g) \text { for all } f, g \in \mathrm{S} .
$$

Then we have the following result (due to [102], we give a different proof which is simpler and more in line with the setup of Chapter 4).

Proposition A.19 Let $s: \mathrm{S} \times \mathrm{S} \longrightarrow \mathbb{R}$ be a real symmetric positive bilinear form and define

$$
\phi_{s}(f)=\mathrm{e}^{-s(f, f) / 4}, \quad f \in \mathrm{S} .
$$

Then $\phi_{s}$ is the generating functional of a state on $\mathfrak{A}(\mathrm{S}, \sigma)$ if and only if $s \in \mathscr{A}$.

Proof. Given a bilinear form $s$ satisfying (A.25) define the kernel $\varphi: \mathrm{S} \times$ $\mathrm{S} \longrightarrow \mathbb{C}$ by

$$
\varphi(f, g)=-\frac{1}{2} \sigma(f, g)+\frac{1}{4} s(f-g, f-g), \quad f, g \in \mathrm{S} .
$$

Then by Schoenberg's theorem $\varphi$ is negative-definite if and only if $\mathrm{e}^{-t \varphi}$ is positive-definite for all $t \geq 0$. Let $n \in \mathbb{N}$ and $f_{1}, \ldots, f_{n} \in \mathbf{S}$, and $z_{1}, \ldots, z_{n} \in \mathbb{C}$, write $z_{i}=a_{i}+\mathrm{i} b_{i}, i=1, \ldots, n$, and suppose that $\sum z_{i}=0$. Then it follows that

$$
\begin{aligned}
\sum_{i, j=1}^{n} z_{i} \bar{z}_{j} \varphi\left(f_{i}, f_{j}\right)= & \sum_{i, j=1}^{n}\left(a_{i}+\mathrm{i} b_{i}\right)\left(a_{j}-\mathrm{i} b_{j}\right)\left(-\frac{\mathrm{i}}{2} \sigma\left(f_{i}, f_{j}\right)\right. \\
& \left.\quad+\frac{1}{4}\left[s\left(f_{i}, f_{i}\right)+s\left(f_{j}, f_{j}\right)-2 s\left(f_{i}, f_{j}\right)\right]\right) \\
= & \sigma(f, g)-\frac{1}{2}(s(f, f)+s(g, g)) \\
\leq & \sigma(f, g)-\sqrt{s(f, f) s(g, g)} \\
\leq & 0
\end{aligned}
$$

by the assumption. Here we wrote $f=\sum a_{i} f_{i}$ and $g=\sum b_{i} f_{i}$. Thus $\varphi$ is negative-definite and it follows that $\phi_{\omega}$ satisfies (A.11), hence is a generating functional. Conversely, suppose that $\phi_{s}$ is a generating functional. 
Then it follows that the kernel $\mathrm{e}^{-\varphi}$ is positive-definite, and by bilinearity of $\sigma$ and $s$ and a judicious choice of the $f_{1}, \ldots, f_{n}$ it follows that $\mathrm{e}^{-t \varphi}$ is positive-definite as well for any $t \geq 0$, hence $\varphi$ is negative-definite. By the above calculation we can then conclude that

$$
\begin{aligned}
0 & \geq \sigma(f, g)-\frac{1}{2}(s(f, f)+s(g, g)) \\
& \geq \sigma(f, g)-\sqrt{s(f, f) s(g, g)}
\end{aligned}
$$

for all $f, g \in \mathrm{S}$, which implies (A.25).

Hence every $s \in \mathscr{A}$ determines a generating functional $\phi_{s}$ which by Proposition A.9 induces a state $\omega_{s}$ on $\mathfrak{A}(\mathrm{S}, \sigma)$.

Definition A.20 The states $\omega_{s}$ for $s \in \mathscr{A}$ are called quasifree states.

Since the map

$$
\mathbb{R} \ni t \mapsto \phi_{A}(t f+g)=\exp \left[\frac{t^{2}}{4} s_{A}(f, f)+2 t s_{A}(f, g)+\frac{1}{4} s_{A}(g, g)\right]
$$

is continuous for all $f, g \in \mathrm{S}$ it follows from Proposition A.13 that every quasifree state is regular. We remark [102] that if $(\mathrm{S}, \sigma)$ is a symplectic space with nondegenerate $\sigma$ the Fock states (i.e. the states contained in the folium of the Fock representation) are precisely those quasifree states $\omega_{J}$ induced by $s_{J} \in \mathscr{A}$, where $s_{J}(f, f)=\sigma(J f, f)$ for all $f \in \mathrm{S}$, with $J$ such that $J^{+}=-J$ (adjoint with respect to $\sigma$ ) and $J^{2}=-\mathbb{1}$. Then $J$ permits us to introduce a complex structure on S, by defining $(\lambda+\mathrm{i} \mu) f=\lambda f+\mu J(f)$, where $\lambda, \mu \in \mathbb{R}$ and $f \in \mathrm{S}$, and an inner product $\langle f, g\rangle=\sigma(f, J g)+\mathrm{i} \sigma(f, g)$. The generating functional then becomes

$$
\phi_{J}=\exp \left(-\frac{1}{4} s_{J}(f, f)\right)=\exp \left(-\frac{1}{4}\|f\|^{2} \|\right), \quad f \in \mathrm{S} .
$$

Finally, we say that a representation $\pi$ is quasifree if there exists a quasifree state $\omega$ such that its GNS representation $\pi_{\omega}$ agrees with $\pi$. Condition (A.25) shows that $s \in \mathscr{A}$ is an innser product on S. Denote the completion of $\mathrm{S}$ with respect to this inner product by $\tilde{\mathrm{S}}$. It can be shown [102] that the representation $\pi$ induced by a quasifree state $\omega_{s}$, where $s \in \mathscr{A}$, is primary if and only if the continuous extension of $\sigma$ to $\tilde{S}$ is nondegenerate. 


\section{Appendix B.}

\section{Extension of Semigroups}

This Appendix is devoted to a result on the extension of semigroups that we need in Chapter 4 . Let $\mathfrak{A}$ be a $\mathrm{C}^{*}$-algebra and suppose that $\left\{\tau_{t}\right\}_{t \geq 0}$ is a one-parameter semigroup of (completely) positive contractive and unital operators on $\mathfrak{A}$. Moreover, let $\pi$ be a representation of $\mathfrak{A}$ on a Hilbert space $\mathscr{H}$ and let $\mathfrak{M}=\pi(\mathfrak{A}(\mathrm{S}))^{\prime \prime}$ be the von Neumann algebra corresponding to $\pi$. In this Appendix we address the following problem: Does there exist a quantum dynamical semigroup $\left\{T_{t}\right\}_{t \geq 0}$ on $\mathfrak{M}$, i. e. a semigroup of normal unital and (completely) positive operators such that the map $\mathbb{R}^{+} \ni t \mapsto T_{t}(x)$ is ultraweakly continuous for each $x \in \mathfrak{M}$, and such that the condition

$$
T_{t}(\pi(x))=\pi\left(\tau_{t}(x)\right), \quad x \in \mathfrak{A}, t \geq 0
$$

is satisfied?

This problem consists of two parts, the first is that of ultraweak continuity of the map $\mathbb{R}^{+} \ni t \mapsto T_{t}(x)$ and the second is that of normality (i.e. ultraweak-ultraweak continuity) of each $T_{t}$. For automorphism groups the first part of this problem (the second is automatic for *-automorphisms) has been considered by a number of authors $[33,83,104,116]$ : If $\mathfrak{A}$ is a concrete $\mathrm{C}^{*}$-algebra acting on a Hilbert space and $\left\{\alpha_{g}\right\}_{g \in G} \subseteq$ Aut $\mathfrak{A}$ is a group of automorphisms (here $G$ is a topological group) such that each $\alpha_{g}$ extends to an automorphism $\bar{\alpha}_{g}$ on $\mathfrak{M}=\mathfrak{A}^{\prime \prime}$, these authors gave sufficient conditions for $\left\{\bar{\alpha}_{g}\right\}_{g \in G}$ to be ultraweakly continuous provided $G \ni g \mapsto \alpha_{g}(x)$ is ultraweakly continuous for each $x \in \mathfrak{A}$. In Section B.1 we provide a solution of a corresponding problem for weak* continuous one-parameter semigroups on Banach spaces since in the case of a oneparameter semigroup one can, using the Hille-Yosida theorem, obtain a proof without using $\mathrm{C}^{*}$ - or von Neumann algebra techniques. Then in section B.2 this result is applied to the above mentioned problem of extending semigroups acting on a concrete $\mathrm{C}^{*}$-algebra to quantum dynamical semigroups on the von Neumann closure of the $\mathrm{C}^{*}$-algebra. 


\section{B.1. Extension of Semigroups on Banach Spaces}

Let $\mathrm{X}$ be a Banach space. We assume that it has a predual space $\mathrm{X}_{*}$, i. e. a Banach space such that its dual equals $X$. The canonical dual pairing between $\mathbf{X}$ and $\mathbf{X}_{*}$ is denoted by $\langle\cdot, \cdot\rangle$. We shall use the $\sigma\left(\mathbf{X}, \mathbf{X}_{*}\right)$ topology or weak* topology on $X$. A semigroup $\left\{T_{t}\right\}_{t \geq 0}$ of $\|\cdot\|$-bounded operators is called weak ${ }^{*}$ continuous if the maps $\mathbb{R}^{+} \ni t \mapsto T_{t}(x)$ for each $x \in \mathrm{X}$ and $x \mapsto T_{t}(x)$ for each $t \geq 0$ are continuous when $\mathrm{X}$ is endowed with this topology. We define its weak ${ }^{*}$ generator $Z$ as usual:

$$
\begin{aligned}
\operatorname{dom} Z & =\left\{x \in \mathrm{X}: \lim _{t \downarrow 0} t^{-1}\left(T_{t}(x)-x\right) \text { in the weak }{ }^{*} \text { topology }\right\} \\
Z x & =\lim _{t \downarrow 0} t^{-1}\left(T_{t}(x)-x\right) \text { in the weak* topology, } \quad x \in \operatorname{dom} Z .
\end{aligned}
$$

We recall the Hille-Yosida theorem which is the basis of the proof of the main result in this section (Theorem B.4).

Theorem B.1 Let $Z$ be an operator on $\mathrm{X}$, then the following assertions are equivalent:

1. The operator $Z$ is the generator of a weak* continuous contractive semigroup.

2. The operator $Z$ is weak* densely defined and weak ${ }^{*}-w e a k^{*}$ closed. For all numbers $\lambda \geq 0$

$$
\|(\lambda \mathbb{1}-Z) x\| \geq \lambda\|x\| \quad \text { for all } x \in \operatorname{dom} Z,
$$

and for some and hence all $\lambda>0$

$$
\operatorname{ran}(\lambda \mathbb{1}-Z)=X
$$

If 1. or 2. holds we have the following integral representation of the resolvent $R_{\lambda}(Z)$ of $Z$,

$$
R_{\lambda}(Z) x=(\lambda \mathbb{1}-Z)^{-1} x=\int_{0}^{\infty} \mathrm{e}^{-\lambda s} T_{s}(x) \mathrm{d} s, \quad x \in \mathrm{X}, \operatorname{Re} \lambda>0,
$$

where the integral converges in the weak* topology.

For a proof see [6] (Proposition 3.1.6. and Theorem 3.1.10.).

For the rest of this section we shall always assume that the following three conditions hold true: 
1. The predual $\mathrm{X}_{*}$ is separable.

2. There exists a subspace $X_{0} \subseteq X$ such that ball $X_{0} \subseteq$ ball $X$ is weak* dense (then clearly $X_{0}$ is weak* dense in $X$ ).

Let $\left\{T_{t}^{0}\right\}_{t \geq 0}$ be a semigroup of contractive operators $T_{t}^{0}: \mathrm{X}_{0} \longrightarrow \mathrm{X}_{0}$. In addition to 1 . and 2 . above we also assume:

3. Each operator $T_{t}^{0}, t \geq 0$, has an extension to a weak*-weak* continuous operator $T_{t}: \mathrm{X} \longrightarrow \mathrm{X}$.

It is clear that the extension $T_{t}$ is unique and contractive. Our goal is to prove that under these assumptions $\left\{T_{t}\right\}_{t \geq 0}$ is a weak* continuous semigroup on $\mathrm{X}$. We start by establishing two lemmas.

Lemma B.2 Assume that $t \mapsto T_{t}(x)=T_{t}^{0}(x)$ is weak measurable for $^{*}$ each $x \in \mathrm{X}_{0}$ and define the family of operators $\left\{R_{\lambda}^{0}: \lambda>0\right\}$ by

$$
R_{\lambda}^{0}(x)=\int_{0}^{\infty} \mathrm{e}^{-\lambda s} T_{s}^{0}(x) \mathrm{d} s, \quad \text { where } x \in \mathrm{X}_{0}, \lambda>0 .
$$

Then $R_{\lambda}^{0}$ extends to a bounded weak ${ }^{*}-$ weak ${ }^{*}$ continuous operator $R_{\lambda}$ on $\mathrm{X}$ such that $\left\|R_{\lambda}\right\| \leq 1 / \lambda$ for all $\lambda>0$.

Proof. The separability of $\mathbf{X}_{*}$ implies that the weak* topology on ball $\mathbf{X}$ is metrizable and by assumption ball $\mathrm{X}_{0} \subseteq$ ball $\mathrm{X}$ is dense. Let $x \in$ ball $\mathrm{X}$ and choose $\left\{x_{n}\right\}_{n \in \mathbb{N}} \subseteq$ ball $\mathrm{X}_{0}$ with $x_{n} \rightarrow x$. As pointwise limit of the maps $t \mapsto \alpha_{n}(t)=T_{t}^{0}\left(x_{n}\right)$ the map $t \mapsto \alpha(t)=T_{t}(x)$ is weak* measurable and we may define

$$
R_{\lambda}(x)=\int_{0}^{\infty} \mathrm{e}^{-\lambda s} T_{s}(x) \mathrm{d} s
$$

for all $x \in \mathrm{X}$ and $\lambda>0$, the integral is taken to be a weak* integral; then clearly $R_{\lambda}$ extends $R_{\lambda}^{0}$. Next we have

$$
\left|\left\langle R_{\lambda}(x), \varphi\right\rangle\right| \leq \int_{0}^{\infty} \mathrm{e}^{-\lambda s}\|x\| \cdot\|\varphi\| \mathrm{d} s \leq \frac{1}{\lambda}\|x\| \cdot\|\varphi\|
$$

for all $x \in \mathrm{X}$ and $\varphi \in \mathrm{X}_{*}$, thus $\left\|R_{\lambda}\right\| \leq 1 / \lambda$. By the dominated convergence theorem we obtain for $\varphi \in \mathrm{X}_{*}$ that

$$
\begin{aligned}
\left\langle R_{\lambda}\left(x_{n}\right), \varphi\right\rangle & =\int_{0}^{\infty} \mathrm{e}^{-\lambda s}\left\langle T_{s}\left(x_{n}\right), \varphi\right\rangle \mathrm{d} s \\
& \rightarrow \int_{0}^{\infty} \mathrm{e}^{-\lambda s}\left\langle T_{s}(x), \varphi\right\rangle \mathrm{d} s=\left\langle R_{\lambda}(x), \varphi\right\rangle
\end{aligned}
$$


as $n \rightarrow \infty$ for any sequence $\left\{x_{n}\right\}_{n \in \mathbb{N}} \subseteq$ ball $\mathrm{X}$ with $x_{n} \rightarrow x$, therefore $R_{\lambda}$ is weak ${ }^{*}{ }_{\text {-weak }}{ }^{*}$ continuous when restricted to ballX. Using the KreinŠmulian theorem this implies that $R_{\lambda}$ is weak ${ }^{*}{ }_{-}$weak $^{*}$ continuous on X. $\square$

Lemma B.3 The operators $R_{\lambda}$ defined in Lemma B.2 satisfy the resolvent equation

$$
R_{\lambda}-R_{\mu}=(\mu-\lambda) R_{\lambda} R_{\mu} \quad \text { for all } \lambda, \mu>0 .
$$

Proof. Assume that $\lambda-\mu>0$. Using (B.5) we obtain

$$
\begin{aligned}
R_{\lambda} R_{\mu}(x) & =\int_{0}^{\infty} \int_{0}^{\infty} \mathrm{e}^{-\lambda s} \mathrm{e}^{-\mu t} T_{t+s}(x) \mathrm{d} t \mathrm{~d} s \\
& =\int_{0}^{\infty} \mathrm{e}^{-(\lambda-\mu) s}\left(\int_{s}^{\infty} \mathrm{e}^{-\mu t} T_{t}(x) \mathrm{d} t\right) \mathrm{d} s \\
& =\frac{1}{\lambda-\mu} R_{\mu}(x)-\int_{0}^{\infty} \mathrm{e}^{-(\lambda-\mu) s}\left(\int_{0}^{s} \mathrm{e}^{-\mu t} T_{t}(x) \mathrm{d} t\right) \mathrm{d} s \\
& =\frac{1}{\lambda-\mu}\left(R_{\mu}(x)-R_{\lambda}(x)\right)
\end{aligned}
$$

by a partial integration. From $\left[R_{\lambda}, R_{\mu}\right]=0$ for all $\lambda, \mu>0$ the result follows.

We are now ready to prove the main result of this appendix.

Theorem B.4 Suppose that conditions 1.-3. hold true. Then if $t \mapsto$ $T_{t}(x)=T_{t}^{0}(x)$ is weak ${ }^{*}$ continuous for all $x \in \mathrm{X}_{0}$ it follows that $\left\{T_{t}\right\}_{t \geq 0}$ is a weak* continuous contractive semigroup on $\mathrm{X}$.

Proof. Using (B.6) we conclude that for any $\lambda, \mu>0$ the following relations hold:

$$
\text { ker } R_{\lambda}=\operatorname{ker} R_{\mu}, \quad \operatorname{ran} R_{\lambda}=\operatorname{ran} R_{\mu} .
$$

Now let $\epsilon>0$ and choose $x \in \mathrm{X}_{0}, \varphi \in \mathrm{X}_{*}$. Then there exists $\delta>0$ such that $0 \leq t<\delta$ implies

$$
\left|\left\langle T_{t}(x)-x, \varphi\right\rangle\right|=\left|\left\langle T_{t}^{0}(x)-x, \varphi\right\rangle\right|<\epsilon .
$$

Furthermore, there exists $\lambda_{0} \geq 0$ such that $\lambda>\lambda_{0}$ implies

$$
\lambda \int_{\delta}^{\infty} \mathrm{e}^{-\lambda s} \mathrm{~d} s=\mathrm{e}^{-\lambda \delta}<\epsilon .
$$


Thus if $\lambda>\lambda_{0}$ it follows that

$$
\begin{aligned}
\left|\left\langle\lambda R_{\lambda}(x)-x, \varphi\right\rangle\right| \leq & \lambda \int_{0}^{\delta} \mathrm{e}^{-\lambda s}\left|\left\langle T_{s}(x)-x, \varphi\right\rangle\right| \mathrm{d} s \\
& +\lambda \int_{\delta}^{\infty} \mathrm{e}^{-\lambda s}\left|\left\langle T_{s}(x)-x, \varphi\right\rangle\right| \mathrm{d} s \\
\leq & \epsilon \lambda \int_{0}^{\delta} \mathrm{e}^{-\lambda s} \mathrm{~d} s+2 \lambda\|x\| \cdot\|\varphi\| \int_{\delta}^{\infty} \mathrm{e}^{-\lambda s} \mathrm{~d} s \\
& <\epsilon+2 \epsilon\|x\| \cdot\|\varphi\|,
\end{aligned}
$$

which shows that $\lambda R_{\lambda}(x) \rightarrow x$ as $\lambda \rightarrow \infty$ in the weak* topology for any $x \in \mathrm{X}_{0}$. Since $\left\|\lambda R_{\lambda}\right\| \leq 1$ it follows from (B.7) that

$$
\text { ball } \mathrm{X}_{0} \subseteq \overline{\text { ball ran } R_{\lambda}} \subseteq \text { ball } \mathrm{X},
$$

implying that ball $\operatorname{ran} R_{\lambda}$ is dense in ballX. Next from (B.6) we conclude that

$$
\begin{aligned}
\left\|\left(\lambda R_{\lambda}-\mathbb{1}\right) R_{\mu}\right\| & =\left\|\lambda R_{\lambda} R_{\mu}-R_{\lambda}+(\mu-\lambda) R_{\lambda} R_{\mu}\right\| \\
& \leq\left\|R_{\lambda}\right\|+\mu\left\|R_{\lambda}\right\| \cdot\left\|R_{\mu}\right\| \\
& \leq \frac{2}{\lambda}
\end{aligned}
$$

for all $\lambda>0$. Thus $\left\|\left(\lambda R_{\lambda}-\mathbb{1}\right) x\right\| \leq(2 / \lambda)\|x\|$ for all $x \in \operatorname{ran} R_{\lambda}$. For each $x \in \mathrm{X}$ there exists a $\|\cdot\|$-bounded sequence $\left\{x_{n}\right\}_{n \in \mathbb{N}} \subseteq \operatorname{ran} R_{\lambda}$ with $\left\|x_{n}\right\| \leq\|x\|$ such that $\lim x_{n}=x$ in relative to the weak* topology. Then, for any $\varphi \in X_{*}$, we have

$$
\left|\left\langle\left(\lambda R_{\lambda}-\mathbb{1}\right) x_{n}, \varphi\right\rangle\right| \leq \frac{2}{\lambda}\|x\| \cdot\|\varphi\|,
$$

and upon letting $n \rightarrow \infty$ we get $\left|\left\langle\left(\lambda R_{\lambda}-\mathbb{1}\right) x, \varphi\right\rangle\right| \leq(2 / \lambda)\|x\| \cdot\|\varphi\|$, and finally

$$
\left\|\left(\lambda R_{\lambda}-\mathbb{1}\right) x\right\| \frac{2}{\lambda}\|x\|
$$

for all $x \in \mathrm{X}$. This proves that

$$
\lim _{\lambda \rightarrow \infty} \lambda R_{\lambda} x=x
$$

for all $x \in \mathrm{X}$ in the norm topology. If $x \in \operatorname{ker} R_{\lambda}$ for some and hence all $\lambda>0$ then this result implies $0=\lim _{\lambda \rightarrow \infty} \lambda R_{\lambda} x=x$, hence $\operatorname{ker} R_{\lambda}=\{0\}$ and $R_{\lambda}$ is injective for any $\lambda>0$. This allows us to define the operator

$$
Z=\lambda_{0} \mathbb{1}-R_{\lambda_{0}}^{-1}, \quad \operatorname{dom} Z=\operatorname{ran} R_{\lambda_{0}}
$$


for some $\lambda_{0}>0$. Using (B.6) we have

$$
\begin{aligned}
(\lambda \mathbb{1}-Z) R_{\lambda} & =\left[\left(\lambda-\lambda_{0}\right) \mathbb{1}+\left(\lambda_{0} \mathbb{1}-Z\right)\right] R_{\lambda} \\
& =\left[\left(\lambda-\lambda_{0}\right) \mathbb{1}+\left(\lambda_{0} \mathbb{1}-Z\right)\right] R_{\lambda_{0}}\left[\mathbb{1}-\left(\lambda-\lambda_{0}\right) R_{\lambda}\right] \\
& =\mathbb{1}+\left[\left(\lambda-\lambda_{0}\right)\left[R_{\lambda_{0}}-R_{\lambda}-\left(\lambda-\lambda_{0}\right) R_{\lambda} R_{\lambda_{0}}\right]=\mathbb{1},\right.
\end{aligned}
$$

and similarly also $R_{\lambda}(\lambda \mathbb{1}-Z)=\mathbb{1}$. This shows that $R_{\lambda}$ is the resolvent of $Z$ and that the definition of $Z$ does not depend on $\lambda_{0}$. Moreover, $Z$ is weak* densely defined and weak ${ }^{*}{ }_{-}{ }_{\text {weak }}{ }^{*}$ closed since $R_{\lambda}$ is weak ${ }^{*}{ }_{-}$weak $^{*}$ continuous. Taken together we have

$$
\operatorname{ran}(\lambda \mathbb{1}-Z)=\mathrm{X} \quad \text { and } \quad\|(\lambda \mathbb{1}-Z) x\| \geq \lambda\|x\|
$$

for all $x \in \operatorname{dom} Z, \lambda>0$, showing that condition 2. of Theorem B.1 is satisfied and $Z$ is the generator of a weak* continuous contractive semigroup $\left\{S_{t}\right\}_{t \geq 0}$. It remains to prove that $\left\{S_{t}\right\}_{t \geq 0}$ coincides with $\left\{T_{t}\right\}_{t \geq 0}$. By the integral representation of the resolvent

$$
\int_{0}^{\infty} \mathrm{e}^{-\lambda s} S_{s}(x) \mathrm{d} s=R_{\lambda}(x)=\int_{0}^{\infty} \mathrm{e}^{-\lambda s} T_{s}(x) \mathrm{d} s
$$

for $x \in \mathrm{X}$ and all $\lambda \in \mathbb{C}$ with $\operatorname{Re} \lambda>0$, which implies equality of $\left\{T_{t}\right\}_{t \geq 0}$ and $\left\{S_{t}\right\}_{t \geq 0}$ because the integrands are continuous functions of $s$.

\section{B.2. Extension of Semigroups on von Neumann Algebras}

We now apply the result of the last section to semigroups on von Neumann algebras. Recall that on a von Neumann algebra $\mathfrak{M}$ with predual space $\mathfrak{M}_{*}$ the weak* topology, i. e. the $\sigma\left(\mathfrak{M}, \mathfrak{M}_{*}\right)$ topology, is equivalent to the ultraweak topology, and that an operator $T: \mathfrak{M} \longrightarrow \mathfrak{M}$ is normal if and only if it is $\sigma\left(\mathfrak{M}, \mathfrak{M}_{*}\right)-\sigma\left(\mathfrak{M}, \mathfrak{M}_{*}\right)$ continuous.

Recall (Definition 1.16) that a semigroup $\left\{T_{t}\right\}_{t \geq 0}$ of operators on a von Neumann algebra $\mathfrak{M}$ is called a quantum dynamical semigroup if it is weak* continuous, i. e. if each $T_{t}$ is continuous and if $t \mapsto T_{t}(x)$ is ultraweakly continuous for all $x \in \mathfrak{M}$, and moreover if each operator $T_{t}$ is completely positive and unital, i.e. $T_{t}(\mathbb{1})=\mathbb{1}$ for any $t \geq 0$.

Let $\mathfrak{B}$ be a nondegenerate ${ }^{*}$-subalgebra on the Hilbert space $\mathscr{H}(\mathfrak{B}$ is automatically nondegenerate if it contains the identity operator $\mathbb{1}$ ), and let $\mathfrak{M}=\mathfrak{B}^{\prime \prime}$, the von Neumann algebra generated by $\mathfrak{B}$; then $\mathfrak{B}$ is ultraweakly dense in $\mathfrak{M}$. We consider a contractive semigroup $\left\{T_{t}^{0}\right\}_{t \geq 0}$ on $\mathfrak{B}$. 
Corollary B.5 Assume that $\mathscr{H}$ is separable and that each $T_{t}^{0}, t \geq 0$, extends to a normal operator $T_{t}$ on $\mathfrak{M}$. Moreover, assume that $\mathbb{R}^{+} \ni t \mapsto$ $T_{t}(x)$ is ultraweakly continuous for each $x \in \mathfrak{B}$. Then $\left\{T_{t}\right\}_{t \geq 0}$ is a weak ${ }^{*}$ continuous contractive semigroup on $\mathfrak{M}$.

Proof. Since $\mathscr{H}$ is separable, it follows that $\mathfrak{M}_{*}$ is separable [24]. Moreover, by the Kaplansky density theorem ball $\mathfrak{B} \subseteq$ ball $\mathfrak{M}$ is weak* dense and the conclusion follows from Theorem B.4.

Thus if $T_{t}^{0}$ in Corollary B.5 is unital then it follows that the semigroup $\left\{T_{t}\right\}_{t \geq 0}$ is positive.

Now let $\mathfrak{A}$ be an abstract $\mathrm{C}^{*}$-algebra and let $\left\{\tau_{t}\right\}_{t \geq 0}$ be a contractive semigroup on $\mathfrak{A}$. For a state $\omega$ on $\mathfrak{A}$ let us consider the GNS representation $\left(\pi_{\omega}, \mathscr{H}_{\omega}, \xi_{\omega}\right)$ of $\mathfrak{A}$ on the Hilbert space $\mathscr{H}_{\omega}$ with cyclic vector $\xi_{\omega} \in \mathscr{H}_{\omega}$.

Definition B.6 The semigroup $\left\{\tau_{t}\right\}_{t \geq 0}$ is called $\omega$-continuous if $t \mapsto$ $\pi_{\omega}\left(\tau_{t}(x)\right)$ is ultraweakly continuous for all $x \in \mathfrak{A}$. Moreover, we say that $\left\{\tau_{t}\right\}_{t \geq 0}$ is $\omega$-covariant if there is a semigroup of normal contractive operators $\left\{T_{t}\right\}_{t \geq 0}$ on $\mathfrak{M}$ such that

$$
T_{t}\left(\pi_{\omega}(x)\right)=\pi_{\omega}\left(\tau_{t}(x)\right), \quad x \in \mathfrak{A}, t \geq 0 .
$$

Let us observe that $\left\{T_{t}\right\}_{t \geq 0}$ is uniquely defined. Furthermore, $\left\{T_{t}\right\}_{t \geq 0}$ is positive (resp. completely positive) if and only if $\left\{\tau_{t}\right\}_{t \geq 0}$ positive (resp. completely positive), so this concept also applies to quantum dynamical semigroups. A state $\omega$ is called separating if $\xi_{\omega}$ is a separating vector for the von Neumann algebra $\mathfrak{M}=\pi_{\omega}(\mathfrak{A})^{\prime \prime}$, or equivalently, if $\xi_{\omega}$ is cyclic for $\mathfrak{M}^{\prime}$. Let $N_{\omega} \subseteq \mathscr{S}(\mathfrak{A})$ be the folium of all $\omega$-normal states.

Theorem B.7 Let $\omega$ be a separating state on $\mathfrak{A}$ and let $\mathscr{H}_{\omega}$ be separable. Suppose that a positive unital semigroup $\left\{\tau_{t}\right\}_{t \geq 0}$ is $\omega$-continuous, and let $\omega$ and $\omega \circ \tau_{t}$ be quasi-equivalent for any $t \geq 0$. Then $\left\{\tau_{t}\right\}_{t \geq 0}$ is $\omega$-covariant and the semigroup $\left\{T_{t}\right\}_{t \geq 0}$ on $\mathfrak{M}$ satisfying (B.9) is positive and weak* continuous.

Proof. If $\omega$ is a separating state it follows that

$$
N_{\omega}=\overline{\{\psi \in \mathscr{S}(\mathfrak{A}): \psi \leq \lambda \omega \text { for some } \lambda \geq 0\}},
$$

where the closure is taken in norm topology (see [63], Theorem 4.2). By assumption we have $N_{\omega}=N_{\omega \circ \tau_{t}}$. Let $\psi \in N_{\omega}$, then there exists a sequence $\left\{\psi_{n}\right\}_{n \in \mathbb{N}}$ with $\psi_{n} \leq \lambda_{n} \omega$ such that $\lambda_{n} \geq 0$ and $\left\|\psi_{n}-\psi\right\| \rightarrow 0$ as $n \rightarrow \infty$. By positivity of $\tau_{t}$ we have $\psi_{n} \circ \tau_{t} \leq \lambda_{n}\left(\omega \circ \tau_{t}\right)$, hence $\psi_{n} \circ \tau_{t} \in N_{\omega \circ \tau_{t}}$, 
and since folia are norm-closed we obtain $\psi \circ \tau_{t} \in N_{\omega \circ \tau_{t}}=N_{\omega}$, thus we have proved $\left(\tau_{t} \circ \omega\right)\left(N_{\omega}\right) \subseteq N_{\omega}$. Therefore if $\varphi \in \mathfrak{M}_{*}$ is a normal state and $\psi(x)=\left\langle\pi_{\omega}(x), \varphi\right\rangle, x \in \mathfrak{A}$, there exists a normal state $T_{t, *}(\varphi) \in \mathfrak{M}_{*}$ such that

$$
\psi\left(\tau_{t}(x)\right)=\left\langle\pi_{\omega}\left(\tau_{t}(x)\right), \varphi\right\rangle=\left\langle\pi_{\omega}(x), T_{t, *}(\varphi)\right\rangle,
$$

thus a bounded positive map $T_{t, *}$ on $\mathfrak{M}_{*}$ is well-defined and its dual $T_{t}$ is normal and satisfies (B.9). We conclude by Corollary B.5 since $\pi_{\omega}(\mathfrak{A})$ is a nondegenerate ${ }^{*}$-algebra and since $\left\{\tau_{t}\right\}_{t \geq 0}$ is $\omega$-continuous.

We remark that it is easy to show that $\alpha \in$ Aut $\mathfrak{A}$ is $\omega$-covariant if and only if $\omega$ and $\omega \circ \alpha$ are quasi-equivalent (use e.g. Theorem 4.2.26. of [6]).

If $\mathfrak{A}$ has a quasilocal structure, states of different normal folia correspond to systems which differ in global properties [75], such as temperature for systems in thermal equilibrium (KMS states on a type III von Neumann algebra at different temperatures are disjoint). So the last result can be interpreted as follows: If an evolution $\left\{\tau_{t}\right\}_{t \geq 0}$ is such that it changes only local properties of the system (i. e. $\omega$ and $\omega \circ \tau_{t}$ remain quasi-equivalent for all $t \geq 0$ and hence $N_{\omega}=N_{\omega \circ \tau_{t}}$ ) then the evolution is $\omega$-covariant and is given by a semigroup of affine maps on the normal states of a single representation. 


\section{Appendix C.}

\section{Nuclear Spaces and Maps}

In this appendix we collect some basic facts concerning nuclear spaces and nuclear operators on general locally convex topological vector spaces and establish some notation. These facts will be used in Section 4.4. We will mainly follow the expositions in $[25,26]$.

\section{C.1. Nuclear Maps}

Recall that a topological vector space $E$ over $\mathbb{R}$ or $\mathbb{C}$ is called a locally convex space (abbreviated l.c.s. in the following) if it is Hausdorff and if any neighborhood of any $x \in E$ contains a convex neighborhood of $x$. Equivalently, $E$ is a l.c.s. if there exists a neighborhood base of 0 consisting of convex sets. A topology on a real or complex vector space $E$ is called locally convex if $E$, equipped with this topology, is a topological vector space such that it has a neighborhood base of 0 consisting of convex sets (without requiring that $E$ is Hausdorff). The quotient $E / M$ of a topological vector space $E$ and a subspace $M$ is Hausdorff if and only if $M$ is closed in $E$, thus $E / \overline{\{0\}}$ is a Hausdorff space, it is called the Hausdorff space associated with $E$; if $E$ is locally convex it is locally convex as well. A locally convex topology on $E$ is determined by a family of seminorms: choose a family $\mathscr{F}_{0}$ of convex circled ${ }^{1}$ and radial $^{2} 0$-neighborhoods such that $\mathscr{F}=\left\{\lambda^{-1} V: \lambda>0, V \in \mathscr{F}_{0}\right\}$ is a 0 -neighborhood base (such a neighborhood base can always be found) and define the seminorms $p_{V}$ as the gauge of $V$, i. e.

$$
p_{V}(x)=\inf \{\lambda>0: x \in \lambda V\}, \quad x \in E .
$$

Conversely, a family of seminorms $\mathscr{P}$ determines a locally convex topology on $E$ as follows: For $p_{1}, \ldots, p_{n} \in \mathscr{P}$ define $V\left(p_{1}, \ldots, p_{n} ; \epsilon\right)=\{x \in E$ :

${ }^{1} \mathrm{~A}$ subset $V \subseteq E$ is called circled if $\lambda V \subseteq V$ for all $\lambda$ with $|\lambda| \leq 1$.

${ }^{2} \mathrm{~A}$ subset $V \subseteq E$ is called radial if for any $x_{1}, \ldots, x_{n} \in E$ there exists $\lambda_{0} \in \mathbb{K}$ such that $\left\{x_{1}, \ldots, x_{n}\right\} \subseteq \lambda V$ whenever $|\lambda| \geq\left|\lambda_{0}\right|$. 
$\left.p_{1}(x) \leq \epsilon, \ldots, p_{n}(x) \leq \epsilon\right\}$, then it can be shown that the collection of all $V\left(p_{1}, \ldots, p_{n} ; \epsilon\right)$ with $\epsilon>0, n \in \mathbb{N}$ and $p_{1}, \ldots, p_{n} \in \mathscr{P}$ is a neighborhood base at 0 for a locally convex topology of $E$.

Now let $E$ be a vector space over $\mathbb{K}$ and let $V \subseteq E$ be a convex circled and radial subset. Then $\left\{n^{-1} V: n \in \mathbb{N}\right\}$ is a neighborhood base for a locally convex topology on $E$. Let $p$ be the gauge of $V$ and consider the quotient $E_{V}=E / \operatorname{ker} p$ where $\operatorname{ker} p=\{x \in E: p(x)=0\}$. It is the Hausdorff space associated with $E$ and it is normable by the norm $\|[x]\|_{V}=p(\underset{\tilde{E}}{x}), x \in E$, where $[x]$ denotes the coset $[x]=x+\operatorname{ker} p$. Its completion $\tilde{E}_{V}$ is then a Banach space. The canonical map $\phi_{V}: E \longrightarrow$ $\tilde{E}_{V}$ is continuous if $E$ is a l.c.s. and if $V$ is a convex circled and radial neighborhood of 0 .

If $E$ is a l.c.s. and if $B \subseteq E$ is a convex circled and bounded subset define the subspace

$$
E_{B}=\bigcup_{n=1}^{\infty} n B .
$$

The gauge $p_{B}$ of $B$ is a norm on $E_{B}$ and the canonical embedding $\psi_{B}$ : $E_{B} \longrightarrow E$ is continuous. If $B$ is complete in $E$ then $E_{B}$ is a Banach space. Note that if $B$ is additionally circled and radial we have $E_{V}=E_{B}$, so our notation is consistent.

Let $V_{1}$ and $V_{2}$ be two convex circled and radial subsets of a real or complex vector space $E$ and let $p_{1}$ and $p_{2}$ be the corresponding gauges of $V_{1}$ and $V_{2}$, respectively. If $V_{1} \subseteq V_{2}$ then $\operatorname{ker} p_{1} \subseteq \operatorname{ker} p_{2}$ and each equivalence class $[x]=x+\operatorname{ker} p$ is contained in a unique equivalence class $[y]=y+\operatorname{ker} p_{2}$. A linear map $E_{V_{1}} \longrightarrow E_{V_{2}}$ is well-defined by $[x] \mapsto[y]$ and continuous. Therefore it has a unique extension $\phi_{V_{2}, V_{1}}: \tilde{E}_{V_{1}} \longrightarrow \tilde{E}_{V_{2}}$ which is called the canonical map from $\tilde{E}_{V_{1}}$ into $\tilde{E}_{V_{2}}$. Similarly, if $B_{1}, B_{2}$ are convex circled and bounded subsets such that $B_{1} \subseteq B_{2}$ and $B_{1} \neq \emptyset$ then $E_{B_{1}} \subseteq E_{B_{2}}$ and the canonical embedding $\psi_{B_{2}, B_{1}}: E_{B_{1}} \longrightarrow E_{B_{2}}$ is continuous. In this situation we have

$$
\phi_{V_{2}}=\phi_{V_{2}, V_{1}} \circ \phi_{V_{1}}, \quad \psi_{C_{2}}=\psi_{C_{2}, C_{1}} \circ \psi_{C_{1}} .
$$

Let $E$ and $F$ be topological vector spaces and $u: E \longrightarrow F$ a linear map. The linear map $u$ is called bounded if there exists a 0-neighborhood $U$ of $E$ such that $u(U) \subseteq F$ is bounded in $F$. If $E$ and $F$ are l.c.s. and if $u$ is bounded we can choose a convex circled and radial 0-neighborhood $U$ of $E$ and a convex circled and bounded subset (take the convex circled hull) $B \subseteq F$ such that $u(U) \subseteq B$. Then there exists a linear map $u_{0}$ : $E_{U} \longrightarrow F_{B}$ such that $u=\psi_{B} \circ u_{0} \circ \phi_{U}$. Moreover, $u_{0}$ is bounded. If $F_{B}$ 
is complete then $u_{0}$ has a continuous (i. e. bounded) extension $\bar{u}_{0}$ to the completion $\tilde{E}_{U}$, i.e. $\bar{u}_{0}$ is a bounded map from the Banach space $\tilde{E}_{U}$ into the Banach space $F_{B}$ such that

$$
u=\psi_{B} \circ \bar{u}_{0} \circ \phi_{U} .
$$

Let $E$ and $F$ be vector spaces, $E^{\prime}$ the algebraic dual of $E$ and $E^{\prime} \odot F$ the algebraic tensor product. We define a map $\phi: E^{\prime} \otimes F \longrightarrow \mathrm{L}(E, F)$ as follows: Let $z=\sum_{i=1}^{n} f_{i} \otimes y_{i} \in E^{\prime} \otimes F$, then

$$
\phi(z) x=\sum_{i=1}^{n} f_{i}(x) y_{i}, \quad \text { for all } x \in E .
$$

Then $\phi$ is a linear injection and its range are the finite rank maps in $\mathrm{L}(E, F)$. Now let $E$ and $F$ be Banach spaces and restrict the embedding $\phi$ to $E^{*} \odot F$, where $E^{*}$ is the Banach space dual of $E$, and equip $E^{*} \odot F$ with the projective cross norm $\gamma$ defined in (1.40). For $z=\sum_{i=1}^{n} f_{i} \otimes y_{i} \in E^{*} \odot F$ we have

$$
\begin{aligned}
\|\phi(z)\| & =\sup \{\|\phi(z) x\|: x \in E,\|x\| \leq 1\} \\
& \leq \sup _{\|x\| \leq 1}\left|\sum_{i=1}^{n} f_{i}(x) y_{i}\right| \\
& \leq \sum_{i=1}^{n}\left\|f_{i}\right\| \cdot\left\|y_{i}\right\|
\end{aligned}
$$

for any representation of $z$, hence by the definition of $\gamma$ we obtain $\|\phi(z)\| \leq$ $\gamma(z)$, hence $\phi$ is bounded. Since $F$ is complete, $\mathrm{L}(E, F)$ is complete for the norm topology, thus it follows that $\phi$ has an extension $\bar{\phi}$ to the completion $E^{*} \otimes_{\gamma} F$ of $E^{*} \odot F$ with respect to $\gamma$. This allows us to introduce the concept of a nuclear operator between Banach spaces $E$ and $F$. A linear operator in $\mathrm{L}(E, F)$ is called nuclear if it is contained in the range $\bar{\phi}\left(E^{*} \otimes_{\gamma} F\right)$ of $\bar{\phi}$. We have the following characterization of nuclear operators between Banach spaces: $u \in \mathrm{L}(E, F)$ is nuclear if and only if there exist sequences $\left\{f_{n}\right\}_{n \in \mathbb{N}} \subseteq E^{*}$ and $\left\{y_{n}\right\}_{n \in \mathbb{N}} \subseteq F$ such that $\left\|f_{n}\right\| \leq 1$ and $\left\|y_{n}\right\| \leq 1$ for any $n \in \mathbb{N}$, and a sequence $\left\{\lambda_{n}\right\}_{n \in \mathbb{N}} \subseteq \mathbb{K}$ such that $\sum_{n=1}^{\infty}\left|\lambda_{n}\right|<\infty$, such that we have

$$
u(x)=\sum_{n=1}^{\infty} \lambda_{n} f_{n}(x) y_{n} \quad \text { for all } x \in E .
$$

We now generalize the concept of nuclear operators to more general spaces. Let $E$ and $F$ now be l.c.s. A linear map $u \in \mathrm{L}(E, F)$ is called 
nuclear if there exists a convex circled and radial 0-neighborhood $U$ in $E$ and a convex circled and bounded subset $B$ of $F$ with $F_{B}$ complete such that $u(U) \subseteq B$ and such that the operator $\bar{u}_{0} \in \mathrm{L}\left(\tilde{E}_{U}, F_{B}\right)$ in (C.4) is nuclear. We have the following characterization of nuclear operators.

Theorem C.1 Let $E$ and $F$ be l.c.s., then $u \in \mathrm{L}(E, F)$ is nuclear if and only if there exist sequences $\left\{f_{n}\right\}_{n \in \mathbb{N}} \subseteq E^{*}$ such that $\left\{f_{n}\right\}_{n \in \mathbb{N}}$ is an equicontinuous set, a sequence $\left\{y_{n}\right\}_{n \in \mathbb{N}}$ contained in a convex circled and bounded subset $B \subseteq F$ such that $F_{B}$ is complete, and a sequence $\left\{\lambda_{n}\right\}_{n \in \mathbb{N}} \subseteq \mathbb{K}$ satisfying $\sum_{n=1}^{\infty}\left|\lambda_{n}\right|<\infty$, and

$$
u(x)=\sum_{n=1}^{\infty} \lambda_{n} f_{n}(x) y_{n} \quad \text { for all } x \in E .
$$

For a proof see [25]. Clearly, by this theorem the concept of a nuclear operator between l.c.s. generalizes the former in the Banach space case. This theorem is also the key to prove the following properties of nuclear operators given in the following proposition.

Proposition C.2 Let $E$ and $F$ be l.c.s. and let $u \in \mathrm{L}(E, F)$ be nuclear. Then $u$ has the following properties:

1. The operator $u$ is compact (i.e. for a suitable 0 -neighborhood $U$ in $E$ the set $u(U)$ is relatively compact).

2. The operator $u$ has a unique extension to the completion $\tilde{E}$ which is a nuclear operator in $\mathrm{L}(\tilde{E}, F)$.

A proof is given in [25].

\section{C.2. Nuclear Spaces}

In the present section we introduce the concept of a nuclear space. Let $E$ be a l.c.s. Then $E$ is called nuclear if there exists a base $\mathscr{F}$ of convex circled and radial 0-neighborhoods such that for each $V \in \mathscr{F}$ the canonical $\operatorname{map} \phi_{V}: E \longrightarrow \tilde{E}_{V}$ is nuclear.

There are the following alternative characterizations of nuclear spaces.

Proposition C.3 Let E be a l.c.s., then the following assertions are equivalent:

1. E is nuclear. 
2. Every continuous linear map from $E$ into any Banach space is nuclear.

3. For every convex circled and radial 0-neighborhood $U$ in $E$ there exists another convex circled and radial 0-neighborhood $V$ such that $V \subseteq U$ and such that the canonical map $\phi_{U, V}: \tilde{E}_{V} \longrightarrow \tilde{E}_{U}$ is nuclear.

For a proof see [25]. It follows immediately from 2. and Proposition C.2 that the completion $\tilde{E}$ of a nuclear space is nuclear. We can reformulate condition 3. of the previous proposition also in terms of a family of seminorms $\mathscr{P}$ generating the locally convex topology on $E$.

Corollary C.4 Let $E$ be a l.c.s. whose topology is generated by the family $\mathscr{P}$ of seminorms. Then $E$ is nuclear if and only if for every $p \in \mathscr{P}$ there exists $q \in \mathscr{P}$ such that $p \leq q$ and such that the canonical map $\phi_{U, V}: \tilde{E}_{V} \longrightarrow \tilde{E}_{U}$ is nuclear, where $U=\{x \in E: p(x) \leq 1\}$ and $V=\{x \in E: q(x) \leq 1\}$.

Proof. It suffices to note that $U$ and $V$ are convex circled and radial 0neighborhoods in $E$ such that $V \subseteq U$.

Nuclear spaces can also be characterized in terms of Hilbert-Schmidt maps. Let $E$ be a topological vector space and $V \subseteq E$ a convex circled and radial subset. We call $V$ a pre-Hilbert subset if $E_{V}$ is a pre-Hilbert space, i. e. if the norm $\|\cdot\|_{V}$ is induced by a sesquilinear form.

Proposition C.5 Let $E$ be a l.c.s. It is nuclear if and only if the following two conditions are satisfied:

1. There exists a 0-neighborhood base of $E$ consisting of pre-Hilbert subsets.

2. For any pre-Hilbert neighborhood $U$ of $E$ there exists a pre-Hilbert neighborhood $V$ such that $V \subseteq U$ and such that the canonical map $\tilde{E}_{V} \longrightarrow \tilde{E}_{U}$ is Hilbert-Schmidt.

For a proof see $[26]$

We next introduce an important example of a nuclear topology which we shall need in this work.

Proposition C.6 Let $E$ and $F$ be two real or complex vector spaces and $\langle\cdot, \cdot\rangle$ a dual pairing between $E$ and $F$. Then the $\sigma(E, F)$ topology on $E$ is nuclear. 
Proof. A base of convex circled and radial 0-neighborhoods of the $\sigma(E, F)$ topology is given by the sets

$$
V\left(y_{1}, \ldots, y_{n} ; \epsilon\right)=\left\{x \in E:\left|\left\langle x, y_{1}\right\rangle\right|, \ldots,\left|\left\langle x, y_{n}\right\rangle\right|<\epsilon\right\}
$$

where $n \in \mathbb{N}, y_{1}, \ldots, y_{n} \in F$ are linearly independent, and $\epsilon>0$. Choose linearly independent vectors $y_{1}, \ldots, y_{n} \in F$, fix $\epsilon>0$, and define $V=$ $V\left(y_{1}, \ldots, y_{n} ; \epsilon\right)$ and denote the gauge of $V$ by $p$. From linear independence it follows that $\operatorname{ker} p=\left\{x \in E:\left\langle x, y_{1}\right\rangle=\cdots=\left\langle x, y_{n}\right\rangle=0\right\}$. Let, as before, $E_{V}=E / \operatorname{ker} p$ and define the map $\phi_{0}: E \longrightarrow \mathbb{K}^{n}$ by $\phi_{0}(x)=$ $\left(\left\langle x, y_{1}\right\rangle, \ldots,\left\langle x, y_{n}\right\rangle\right)$, moreover, define $\phi: E_{V} \longrightarrow \mathbb{K}^{n}$ by $\phi([x])=\phi_{0}(x)$. This map is clearly well-defined, for if $[x]=\left[x^{\prime}\right]$ then $x-x^{\prime} \in \operatorname{ker} p$ and $\phi_{0}\left(x-x^{\prime}\right)=0$, hence $\phi([x])=\phi\left(\left[x^{\prime}\right]\right)$. Moreover, it follows easily from the definitions that $\phi$ is bijective. Thus we conclude that $\operatorname{dim} E_{V}=\operatorname{dim} \tilde{E}_{V}=$ $n$. Since any linear map between finite-dimensional spaces is nuclear the claim follows from Proposition C.3.

Let $E$ be a topological vector space. A linear map $f: E \longrightarrow \mathscr{H}$ into a Hilbert space $\mathscr{H}$ is called a Hilbert-Schmidt map if there exists a Hilbert space $\mathscr{H}_{0}$ and a continuous map $\alpha: E \longrightarrow \mathscr{H}_{0}$ and a Hilbert-Schmidt map $u: \mathscr{H}_{0} \longrightarrow \mathscr{H}$ such that $f=u \circ \alpha$. The coarsest topology on $E$ such that all Hilbert-Schmidt maps are continuous is called the Sazonov topology on $E$. A 0 -neighborhood base of this topology is given by the sets $f^{-1}(r B)$ where $f$ is a Hilbert-Schmidt map into $\mathscr{H}, B$ is the open unit ball in $\mathscr{H}$ centered at 0 and $r>0$. Notice that the Sazonov topology depends on the original topology on $E$ since the concept of a HilbertSchmidt map does. The next result gives an alternative 0-neighborhood base of the Sazonov topology, its proof is due to [26].

Proposition C.7 Let E be a l.c.s. Then a 0-neighborhood base of the Sazonov topology is given by the family $\mathscr{F}_{\mathrm{S}}$ of all pre-Hilbert subsets such that for each $U \in \mathscr{F}_{\mathrm{S}}$ there exists a pre-Hilbert subset $V \in \mathscr{F}_{\mathrm{S}}$ such that $V \subseteq U$ and such that the canonical map $\tilde{E}_{V} \longrightarrow \tilde{E}_{U}$ is Hilbert-Schmidt.

Proof. Let $f$ be a Hilbert-Schmidt map into a Hilbert space $\mathscr{H}$ and $B$ the open ball centered art 0 in $\mathscr{H}$ of radius $r>0$. Define $U=f^{-1}(B)$ The map $f$ factorizes as $f: E \stackrel{\alpha}{\longrightarrow} \mathscr{H}_{0} \stackrel{u}{\longrightarrow} \mathscr{H}$, where $\alpha$ is continuous and $u$ Hilbert-Schmidt. The set $u^{-1}(B)$ is contained in an open ball $B_{0} \subseteq \mathscr{H}_{0}$ and we put $V=\alpha^{-1}\left(B_{0}\right)$. Then $E_{U}=E / \operatorname{ker} f$ and $E_{V}=E / \operatorname{ker} \alpha$, and ker $f \supseteq \operatorname{ker} \alpha$, thus the canonical map $\phi_{U V}: E_{V} \longrightarrow E_{U}$ is well-defined. We have the injections $\hat{\alpha}: E_{V} \longrightarrow \mathscr{H}_{0}$ and $\hat{f}: E_{U} \longrightarrow \mathscr{H}$, defined by $\hat{\alpha}([x])=\alpha(x)$ and $\hat{f}([x])=f(x), x \in E$. Thus $E_{V}$ and $E_{U}$ inherit 
pre-Hilbert structures from $\mathscr{H}_{0}$ and $\mathscr{H}$, respectively, and $U, V$ are thus pre-Hilbert neighborhoods. Moreover, $V=\alpha^{-1}\left(B_{1}\right) \subseteq \alpha^{-1}\left(u^{-1}(B)\right)=$ $f^{-1}(B)=U$. By noting that the diagram

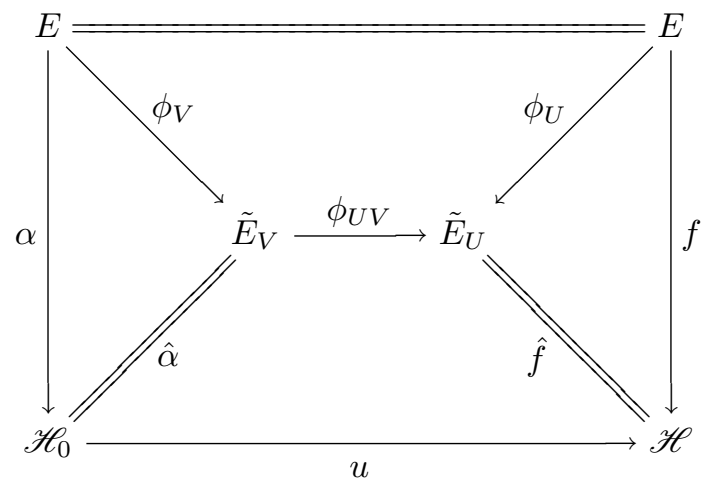

is commutative we see that $\phi_{U V}$ is given by $u$ and is thus Hilbert-Schmidt. Conversely, suppose that $U, V \in \mathscr{F}_{\mathrm{S}}$ such that $V \subseteq U$ and such that the canonical map $\phi_{U V}: \tilde{E}_{V} \longrightarrow \tilde{E}_{U}$ is Hilbert-Schmidt. Since $\phi_{V}: E \longrightarrow$ $\tilde{E}_{V}$ is continuous the map $f:=\phi_{U}=\phi_{U V} \circ \phi_{V}$ is Hilbert-Schmidt. We see that $U=f^{-1}\left(B_{1}\right)$, where $B_{1}$ is the open unit ball centered at 0 in $\tilde{E}_{U}$. $\square$

Corollary C.8 If E is a nuclear l.c.s. then the Sazonov topology on E agrees with the original topology on $E$.

Corollary C.9 Let $E$ be a l.c.s. and equip it with the Sazonov topology. Then $E$ is nuclear.

Proof. Both corollaries immediately follow by combining Proposition C.7 and Proposition C.5. 


\section{Appendix D.}

\section{Cylindrical Measures}

The purpose of this appendix is to collect some results concerning cylindrical measures and provide a precise statement of a generalizations of the classical Minlos-Sazonov theorem which will be used in Chapter 4. The exposition follows partly [8].

\section{D.1. Cylindrical Measures and Functions}

\section{D.1.1. Cylinder Sets}

Let $E$ be a set. We will construct a measurable structure on $E$ by means of a family of maps from $E$ to measurable spaces. Let $I$ be an index set and suppose that for each $i \in I$ there is given a measurable space $\left(E_{i}, \mathscr{B}_{i}\right)$, where $E_{i}$ is some set and $\mathscr{B}_{i}$ a $\sigma$-algebra of subsets of $E_{i}$. Moreover, let $f_{i}: E \longrightarrow E_{i}$ be a surjective map for each $i \in I$. The family $\left\{f_{i}\right\}_{i \in I}$ is called consistent if for any two indices $i_{1}, i_{2} \in I$ there exists $i \in I$ and a pair of surjective measurable maps $\phi_{i_{\alpha} i}: E_{i} \longrightarrow E_{i_{\alpha}}$, where $\alpha=1,2$, such that $f_{i_{\alpha}}=\phi_{i_{\alpha} i} \circ f_{i}$ for $\alpha=1,2$, i. e. such that the diagram

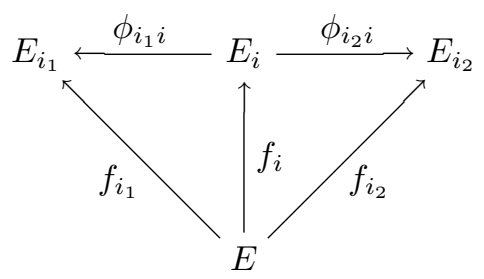

is commutative. In this situation every map $f_{i}$ determines a $\sigma$-algebra $\mathscr{U}_{i}=\left\{f_{i}^{-1}(A): A \in \mathscr{B}_{i}\right\}$ on $E$. By consistency of the maps $\left\{f_{i}\right\}_{i \in I}$ the family of $\sigma$-algebras $\mathscr{U}_{i}$ is directed, i. e. for $i_{1}, i_{2} \in I$ there exists $i \in I$ such that $\mathscr{U}_{i} \supseteq \mathscr{U}_{i_{1}} \cup \mathscr{U}_{i_{2}}$. We write $i_{1} \leq i_{2}$ if $\mathscr{U}_{i_{1}} \subseteq \mathscr{U}_{i_{2}}$. Note that by this definition the index set does not become directed, unlike the set of 
$\sigma$-algebras $\left\{\mathscr{U}_{i}\right\}_{i \in I}$ which is directed by inclusion. By directedness

$$
\mathscr{U}=\bigcup_{i \in I} \mathscr{U}_{i}
$$

is an algebra on $E$, we write $\mathscr{U}=\lim _{I} \mathscr{U}_{i}$ in this situation. The elements of $\mathscr{U}$ are called cylinder sets with respect to the family $\left\{f_{i}\right\}_{i \in I}$ (or simply cylinder sets when no confusion can arise), they are of the form $C_{i}(B)=$ $f_{i}^{-1}(B), i \in I$ and $B \in \mathscr{B}_{i} ; B$ is called the base of the cylinder set $C_{i}(B)$.

Given two spaces $(E, \mathscr{U}),(F, \mathscr{V})$ equipped with algebras $\mathscr{U}$ and $\mathscr{V}$ arising in the way described above, i. e. $\mathscr{U}=\lim _{I} \mathscr{U}_{i}$ and $\mathscr{V}=\lim _{J} \mathscr{V}_{j}$, a map $f: E \longrightarrow F$ is called $\mathscr{U} / \mathscr{V}$-measurable if for all $j \in J$ there exists $i \in I$ such that $f$ is $\mathscr{U}_{i} / \mathscr{V}_{j}$-measurable in the usual sense, i. e. $f^{-1}(B) \in \mathscr{U}_{i}$ for all $B \in \mathscr{V}_{j}$. Moreover, let $(F, \mathscr{F})$ be any measurable space equipped with a $\sigma$-algebra $\mathscr{F}$. A map $f: E \longrightarrow F$ is called a cylindrical map (or a cylindrical function if $F=\mathbb{R}$ or $\mathbb{C}$ ) if there exists an index $i \in I$ such that $f$ is $\mathscr{U}_{i} / \mathscr{F}$-measurable, i. e. if $f^{-1}(B) \in \mathscr{U}_{i}$ for all $B \in \mathscr{F}$.

Now we impose the additional assumption that $E$ is a real linear space (the complex case can be treated analogously). Suppose that $F$ is another real linear space in duality with $E$ via the pairing $\langle\cdot, \cdot\rangle: E \times F \longrightarrow \mathbb{R}$. In this situation it is natural to use its finite-dimensional subspaces to define a cylinder algebra on $E$. Choose some subset $F_{0} \subseteq F$ and let $I$ be the set of ordered finite tuples $\left(y_{1}, \ldots, y_{n}\right)$ of linearly independent elements $y_{1}, \ldots, y_{n}$ of $F_{0}$. For each $i=\left(y_{1}, \ldots, y_{n}\right) \in I$ define the map $f_{i}: E \longrightarrow$ $\mathbb{R}^{n}$ by $f_{i}(x)=\left(\left\langle x, y_{1}\right\rangle, \ldots,\left\langle x, y_{n}\right\rangle\right)$ onto the measurable space $\left(\mathbb{R}^{n}, \mathscr{B}^{n}\right)$, where $\mathscr{B}^{n}$ denotes the Borel $\sigma$-algebra of $\mathbb{R}^{n}$. If now $i_{1}, i_{2} \in I$ where $i_{1}=\left(y_{1}, \ldots, y_{n}\right)$ and $i_{2}=\left(y_{1}^{\prime}, \ldots, y_{m}^{\prime}\right)$, and if we assume that they are related by

$$
y_{j}=\sum_{k=1}^{m} a_{j k} y_{k}^{\prime}, \quad j=1, \ldots, n,
$$

then by defining the matrix $A=\left[a_{j k}\right]$ a linear map $\phi_{i_{1} i_{2}}: \mathbb{R}^{m} \longrightarrow \mathbb{R}^{n}$ is determined which satisfies $f_{i_{1}}=\phi_{i_{1} i_{2}} \circ f_{i_{2}}$. Thus for any two maps $f_{i_{1}}, f_{i_{2}}$ with $i_{1}, i_{2} \in I$ there is always a map $f_{i}$ satisfying the consistency condition, it suffices to take a maximal linearly independent subset $i$ of $i_{1} \cup i_{2}$. As before we have the $\sigma$-algebras $\mathscr{U}_{i}$, the algebra of cylinder sets on $E$ with respect to this family $\left\{f_{i}\right\}_{i \in I}$ of maps will be denoted by $\mathscr{U}_{F_{0}}=\lim _{I} \mathscr{U}_{i}$. It is easy to show that $\mathscr{U}_{F_{0}}$ does not change if $F_{0}$ is replaced by its linear hull, i. e. $\mathscr{U}_{F_{0}}=\mathscr{U}_{\text {lin } F_{0}}$. 


\section{D.1.2. Cylindrical Measures}

Let $E$ be a set endowed with a measurable structure $\mathscr{U}=\lim _{I} \mathscr{U}_{i}$ described in Section D.1.1, i.e. $\mathscr{U}$ is the limit of a directed family of $\sigma$ algebras $\mathscr{U}_{i}$ where $i \in I$. Let $\mu: \mathscr{U} \longrightarrow[0, \infty]$ be a set function. Then $\mu$ is called a cylindrical measure if the restrictions $\mu_{i}$ to $\mathscr{U}_{i}, i \in I$, are $\sigma$ additive. This implies that $\mu$ is finitely additive on $\mathscr{U}$ but, in general, $\mu$ is not $\sigma$-additive. The measures $\left\{\mu_{i}\right\}_{i \in I}$ are called the finite-dimensional measures or finite-dimensional distributions (in probabilistic contexts for normalized positive cylindrical measures) of $\mu$.

Now suppose that $\mathscr{U}=\lim _{I} \mathscr{U}_{i}$ arises from a consistent family of maps $\left\{f_{i}\right\}_{i \in I}$. Choose two indices $i_{1}, i_{2} \in I$ with $i_{1} \leq i_{2}$, then by the consistency condition there exists a map $\phi_{i_{1} i_{2}}: E_{i_{2}} \longrightarrow E_{i_{1}}$ such that $f_{i_{1}}=\phi_{i_{1} i_{2}} \circ f_{i_{2}}$, therefore the cylinder set $C_{i_{1}}(B)=f_{i_{1}}^{-1}(B) \in \mathscr{U}_{i_{1}}$, where $B \in \mathscr{B}_{i_{1}}$, can be written as $C_{i_{1}}(B)=f_{i_{1}}^{-1}(B)=f_{i_{2}}^{-1}\left(\phi_{i_{1} i_{2}}^{-1}(B)\right)=C_{i_{2}}\left(B^{\prime}\right) \in \mathscr{U}_{i_{2}}$ with $B^{\prime}=\phi_{i_{1} i_{2}}^{-1}(B) \in \mathscr{B}_{i_{2}}$, thus the finite-dimensional distributions of $\mu$ satisfy the consistency condition

$$
\mu_{i_{1}}\left(f_{i_{1}}^{-1}(B)\right)=\mu_{i_{2}}\left(f_{i_{2}}^{-1}\left(\phi_{i_{1} i_{2}}^{-1}(B)\right)\right)
$$

for all $i_{1}, i_{2} \in I$ with $i_{1} \leq i_{2}, B \in \mathscr{B}_{i_{1}}$, or by writing $\tilde{\mu}_{i}=\mu_{i} \circ f_{i}^{-1}$, the consistency condition becomes $\tilde{\mu}_{i_{1}}(B)=\tilde{\mu}_{i_{2}}\left(\phi_{i_{1} i_{2}}^{-1}(B)\right)$ for all $i_{1}, i_{2} \in I$ with $i_{1} \leq i_{2}, B \in \mathscr{B}_{i_{1}}$.

Conversely, if for every $i \in I$ a measure $\tilde{\mu}_{i}$ on $\mathscr{B}_{i}$ is given such that the consistency relation $\tilde{\mu}_{i_{1}}(B)=\tilde{\mu}_{i_{2}}\left(\phi_{i_{1} i_{2}}^{-1}(B)\right)$ for all $i_{1}, i_{2} \in I$ with $i_{1} \leq i_{2}$, $B \in \mathscr{B}_{i_{1}}$ is satisfied, there exists a unique cylindrical measure $\mu$ on $(E, \mathscr{U})$ with finite-dimensional distributions $\left\{\mu_{i}\right\}_{i \in I}$, where $\tilde{\mu}_{i}=\mu_{i} \circ f_{i}^{-1}$. Indeed, if $A \in \mathscr{U}$, then there exists $i_{1} \in I$ such that $A=C_{i_{1}}\left(B_{i}\right)=f_{i_{1}}^{-1}\left(B_{i_{1}}\right) \in$ $\mathscr{U}_{i_{1}}$, where $B_{i_{1}} \in \mathscr{B}_{i_{1}}$ is uniquely determined by surjectivity of $f_{i_{1}}$. Then one can define $\mu(A)=\mu_{i_{1}}\left(B_{i_{1}}\right)$. This is well-defined, for if $A \in \mathscr{U}_{i_{2}}$ and hence $A=f_{i_{2}}^{-1}\left(B_{i_{2}}\right)$ with $B_{i_{2}} \in \mathscr{B}_{i_{2}}$, then there exists $i \in I$ with $i_{\alpha} \leq i$, where $\alpha=1,2$, such that $\tilde{\mu}_{i_{1}}\left(B_{i_{1}}\right)=\tilde{\mu}_{i}\left(\phi_{i_{1} i}^{-1}\left(B_{i_{1}}\right)\right)=\tilde{\mu}_{i}\left(\phi_{i_{2} i}^{-1}\left(B_{i_{2}}\right)\right)=$ $\tilde{\mu}_{i_{2}}\left(B_{i_{2}}\right)$.

Given a cylindrical function $f: E \longrightarrow \mathbb{C}$ then we can define the integral of $f$ with respect to a cylindrical measure $\mu$ by

$$
\int_{E} f(x) \mathrm{d} \mu(x):=\int_{E} f(x) \mathrm{d} \mu_{i}(x),
$$

where $i \in I$ is chosen such that $f$ is $\mathscr{U}_{i} / \mathscr{B}^{1}$-measurable and the integral on the right hand side is understood in the usual sense. This is indeed well-defined since by the consistency condition the integral on the right does not depend on the choice of $i$. 
Consider two sets $(E, \mathscr{U}),(F, \mathscr{V})$ with measurable structures $\mathscr{U}=$ $\lim _{I} \mathscr{U}_{i}$ and $\mathscr{V}=\lim _{J} \mathscr{V}_{j}$, and let $\phi: E \longrightarrow F$ be a $\mathscr{U} / \mathscr{V}$-measurable map. Then for any cylindrical measure $\mu$ on $E$ it is easy to see that the set function $\nu:=\mu \circ \phi^{-1}$ on $\mathscr{V}$, defined by $\nu(B)=\mu\left(\phi^{-1}(B)\right)$ for $B \in \mathscr{V}$, is a cylindrical measure on $F$. Let $f: F \longrightarrow \mathbb{C}$ be a cylindrical function. Choose $j \in J$ such that $f$ is $\mathscr{V}_{j} / \mathscr{B}^{1}$-measurable. Then there exists $i \in I$ such that $\phi$ is $\mathscr{U}_{i} / \mathscr{V}_{j}$-measurable, and by the change of variables formula for integrals we have

$$
\int_{E} f(\phi(x)) \mathrm{d} \mu_{i}(x)=\int_{F} f(y) \mathrm{d}\left(\mu_{i} \circ \phi^{-1}\right)(y),
$$

therefore by using the definition (D.2) we get

$$
\int_{E} f(\phi(x)) \mathrm{d} \mu(x)=\int_{F} f(y) \mathrm{d} \nu(y)=\int_{F} f(y) \mathrm{d}\left(\mu \circ \phi^{-1}\right)(y) .
$$

Hence we have obtained a generalization of the change of variables formula for cylindrical measures and cylindrical functions.

Now let $E$ and $F$ be two real vector spaces in duality and consider the cylinder algebra $\mathscr{U}_{F}$ on $E$ described in Section D.1.1. Let $\mu$ be a finite cylindrical measure on $\mathscr{U}_{F}$. Then the function $E \ni x \mapsto \mathrm{e}^{\mathrm{i}\langle x, y\rangle}$ is a bounded cylindrical function for every $y \in F$. Indeed, take $i=(y) \in I$, then the $\sigma$-algebra $\mathscr{U}_{i}$ consists of the sets $\{x \in E:\langle x, y\rangle \in B\}$, where $B \in \mathscr{B}^{1}$, so $x \mapsto \mathrm{e}^{\mathrm{i}\langle x, y\rangle}$ is $\mathscr{U}_{i} / \mathscr{B}^{1}$-measurable. Then the Fourier transform of $\mu$ is defined as the map $\mathcal{F} \mu: F \longrightarrow \mathbb{C}$, where

$$
(\mathcal{F} \mu)(y)=\int_{E} \mathrm{e}^{\mathrm{i}\langle x, y\rangle} \mathrm{d} \mu, \quad y \in F .
$$

It is easy to see that $\mathcal{F} \mu$ is positive-definite for a finite positive cylindrical measure $\mu$. We now turn to the construction of cylindrical measures by means of positive definite functions, i.e. we study generalizations of Bochner's theorem to spaces which are not necessarily locally compact.

Theorem D.1 Let the function $\psi: F \longrightarrow \mathbb{C}$ be continuous on all finitedimensional subspaces $L \subseteq F$ and positive-definite, i. e. given $z_{1}, \ldots, z_{n} \in$ $\mathbb{C}, y_{1}, \ldots, y_{n} \in F$, and $n \in \mathbb{N}$, we have

$$
\sum_{k, \ell=1}^{n} z_{k} \bar{z}_{\ell} \theta\left(y_{k}-y_{\ell}\right) \geq 0 .
$$

Then there exists a unique finite and positive cylindrical measure $\mu$ on $\left(E, \mathscr{U}_{F}\right)$ such that

$$
\psi=\mathcal{F} \mu \text {. }
$$


Conversely, if $\mu$ is a positive finite cylindrical measure on $E$ and $\psi$ is given by (D.6), then it is positive-definite and continuous on finite-dimensional subspaces. If these conditions are satisfied we have $\psi(0)=\mu(E)$.

Proof. If $i \in I$ with $i=\left(y_{1}, \ldots, y_{n}\right)$, where $y_{1}, \ldots, y_{n} \in F$ are linearly independent, consider the finite-dimensional subspace $L=\operatorname{lin}\left\{y_{1}, \ldots, y_{n}\right\}$ and introduce the canonical isomorphism $\phi_{L}: \mathbb{R}^{n} \longrightarrow L$ with

$$
\phi_{L}\left(\alpha_{1}, \ldots, \alpha_{n}\right)=\sum_{i=1}^{n} \alpha_{i} y_{i}, \quad\left(\alpha_{1}, \ldots, \alpha_{n}\right) \in \mathbb{R}^{n}
$$

Since the restriction of $\psi$ to $L$ is continuous there exists by Bochner's theorem a measure $\tilde{\mu}_{i}$ on $\left(\mathbb{R}^{n}, \mathscr{B}^{n}\right)$ such that

$$
\psi(y)=\int_{\mathbb{R}^{n}} \mathrm{e}^{\mathrm{i}\left\langle\tilde{x}, \phi_{L}^{-1}(y)\right\rangle} \mathrm{d} \tilde{\mu}_{i}(\tilde{x}), \quad y \in L \subseteq F .
$$

In order to show that the $\tilde{\mu}_{i}$ define a cylindrical measure $\mu$ on $\left(E, \mathscr{U}_{F}\right)$ it is sufficient to prove that for $i_{1}, i_{2} \in I$ with $i_{1} \leq i_{2}$ we have $\tilde{\mu}_{i_{1}}=\tilde{\mu}_{i_{2}} \circ \phi_{i_{1} i_{2}}^{-1}$ on $\mathscr{B}^{n}$, where $\phi_{i_{1} i_{2}}: \mathbb{R}^{m} \longrightarrow \mathbb{R}^{n}$ as defined before. This, however, is easily established by an application of the change of variables formula. Now if $i \in I$ with $i=\left(y_{1}, \ldots, y_{n}\right)$ and $L=\operatorname{lin}\left\{y_{1}, \ldots, y_{n}\right\}$ it follows that $\left\langle f_{i}(x), \phi_{L}^{-1}(y)\right\rangle=\langle x, y\rangle$ for all $y \in L$, where $f_{i}(x)=\left(\left\langle x, y_{1}\right\rangle, \ldots,\left\langle x, y_{n}\right\rangle\right)$ as before, therefore

$$
\begin{aligned}
\psi(y) & =\int_{\mathbb{R}^{n}} \mathrm{e}^{\mathrm{i}\left\langle\tilde{x}, \phi_{L}^{-1}(y)\right\rangle} \mathrm{d} \tilde{\mu}_{i}(\tilde{x})=\int_{E} \mathrm{e}^{\mathrm{i}\left\langle f_{i}(x), \phi_{L}^{-1}(y)\right\rangle} \mathrm{d} \mu_{i}(x) \\
& =\int_{E} \mathrm{e}^{\mathrm{i}\langle x, y\rangle} \mathrm{d} \mu(x),
\end{aligned}
$$

and the first part of the theorem follows. The converse statement as well as the uniqueness assertion is obvious by Bochner's theorem, the last statement follows from (D.6).

\section{D.2. The Generalized Minlos-Sazonov Theorem}

In this section we will state a generalization of the classical MinlosSazonov theorem for Hilbert spaces which gives a sufficient condition for $\sigma$-additivity of a cylindrical measure. 
Theorem D.2 Let $E$ be a l.c.s. and let $\mu$ be a positive finite cylindrical measure on $\left(E^{\prime}, \mathscr{U}_{E}\right)$, where $E^{\prime}$ denotes the topological dual of $E$. If the Fourier transform $E \ni x \mapsto(\mathcal{F} \mu)(x)$ is continuous at 0 in the Sazonov topology on $E$ then $\mu$ is $\sigma$-additive on $\mathscr{U}_{E}$ and hence there is a unique $\sigma$-additive extension of $\mu$ to the $\sigma$-algebra $\sigma\left(\mathscr{U}_{E}\right)$. Moreover, $\mu$ is strongly regular: For every $\epsilon>0$ there exists a $\sigma\left(E^{\prime}, E\right)$ compact subset $C \subseteq E$ such that $\mu(B)=0$ for every $B \in \mathscr{U}_{E}$ with $B \cap C=\emptyset$.

Next we collect some results concerning weak compactness of families of measures. First let $E$ be a completely regular Hausdorff space and denote by $\mathrm{C}(E)$ the space of continuous functions on $E$. The space of all Radon measures on $E$ is denoted by $\mathscr{M}_{\mathrm{b}}(E)$, and the corresponding duality by $\langle f, \mu\rangle=\int_{E} f \mathrm{~d} \mu$ for $f \in \mathrm{C}(E)$ and $\mu \in \mathscr{M}_{\mathrm{b}}(E)$. By the Riesz-Markov theorem we have $\mathscr{M}_{\mathrm{b}}(E) \cong \mathrm{C}(E)^{*}$. The $\sigma\left(\mathscr{M}_{\mathrm{b}}(E), \mathrm{C}(E)\right)$ topology on $\mathscr{M}_{\mathrm{b}}(E)$ is called the weak topology. By Prokhorov's theorem a subset $K \subseteq \mathscr{M}_{\mathrm{b}}(E)$ is relatively weakly compact if for any $\epsilon>0$ there exists a compact $C \subseteq E$ such that $|\mu|(E \backslash C)<\epsilon$ for all $\mu \in K$. Another sufficient condition for relative weak compactness is stated in the following theorem.

Theorem D.3 Let $E$ be a l.c. s. with topological dual $E^{\prime}$, and let $K \subseteq$ $\mathscr{M}_{\mathrm{b}}^{+}\left(E^{\prime}, \sigma\left(E^{\prime}, E\right)\right)$ be a set of positive normalized Radon measures. If the set of functions $\{\mathcal{F} \mu: \mu \in K\}$ is equicontinuous at 0 in the Sazonov topology corresponding to the $\tau\left(E, E^{\prime}\right)$-topology ${ }^{1}$ on $E$ then $K$ is weakly relatively compact.

We also have a certain converse of Prokhorov's theorem.

Theorem D.4 Let $E$ be a l.c. s. with topological dual $E^{\prime}$, and let $K \subseteq$ $\mathscr{M}_{\mathrm{b}}^{+}\left(E^{\prime}, \sigma\left(E^{\prime}, E\right)\right)$ be a set of positive normalized Radon measures. Suppose that the set of functions $\{\mathcal{F} \mu: \mu \in K\}$ is equicontinuous at 0 in the Sazonov topology corresponding to the $\tau\left(E, E^{\prime}\right)$-topology on $E$. Then for any $\epsilon>0$ there exists a $\sigma\left(E^{\prime}, E\right)$ compact subset $C \subseteq E^{\prime}$ such that $\mu\left(E^{\prime} \backslash C\right)<\epsilon$.

Proofs of these theorems can be found in [8], see also [121].

${ }^{1}$ Here $\tau\left(E, E^{\prime}\right)$ denotes the Mackey topology. 


\section{Bibliography}

\section{Texts and Monographs}

[1] Alicki, R., Lendi, K. Quantum Dynamical Semigroups and Applications. Lecture Notes in Physics Vol. 286, Berlin: Springer (1987).

[2] Araki, H. Mathematical Theory of Quantum Fields Oxford: Oxford Univeristy Press (1999).

[3] Berg, C., Christensen, J.P, R., Ressel, P. Harmonic Analysis on Semigroups. New York: Springer (1984).

[4] Blackadar, B. Operator Algebras. Encyclopaedia of Mathematical Sciences Vol. 122, New York: Springer (2006).

[5] Blanchard, Ph., Giulini, D., Joos, E., Kiefer, C., Stamatescu, I.-O. (eds.) Decoherence: Theoretical, Experimental, and Conceptual Problems. Proceedings, Bielefeld, 1998; Berlin: Springer (2000).

[6] Bratteli, O., Robinson, D. W. Operator Algebras and Quantum Statistical Mechanics I. 2nd ed. New York: Springer (1987).

[7] Bratteli, O., Robinson, D. W. Operator Algebras and Quantum Statistical Mechanics II. New York: Springer (1981).

[8] Dalecky, Yu. L., Fomin, S. V. Measures and Differential Equations in InfiniteDimensional Spaces. Mathematics and its Applications Vol. 76. Dordrecht: Kluwer (1991).

[9] Davies, E. B. Quantum Theory of Open Systems. London: Academic Press (1976).

[10] ЕмсH, G. G. Algebraic Methods in Statistical Mechanics and Quantum Field Theory. New York: Wiley (1972).

[11] Engel, K. J., NAgel, R. One-Parameter Semigroups for Linear Evolution Equations. New York: Springer (2000).

[12] HaAg, R. Local Quantum Physics. 2nd. ed. Berlin: Springer (1996).

[13] Hewitt, E., Ross, K. A. Abstract Harmonic Analysis, Vol. 1. Berlin: Springer (1963).

[14] Hewitt, E., Ross, K. A. Abstract Harmonic Analysis, Vol. 2. Berlin: Springer (1970). 
[15] Joos, E., Zeh, H. D., Kiefer, C., Giulini, D., Kupsch, J., Stamatescu, I.-O. Decoherence and the Appearance of a Classical World in Quantum Theory. 2nd ed. Berlin: Springer (2003).

[16] Kallenberg, O. Foundations of Modern Probability. Berlin: Springer (2002).

[17] Meyer, P. A. Quantum Probability for Probabilists. Lecture Notes in Math. Vol. 1538, Berlin: Springer (1993).

[18] Nagel, R. (ed.) One-Parameter Semigroups of Positive Operators. Lecture Notes in Math. Vol 1184, Berlin: Springer (1986).

[19] Szőkefalvi-Nagy, B., Foiaş, C. Harmonic Analysis of Operators on Hilbert Spaces. Amsterdam: North Holland, Budapest: Akadémiai Kiadó (1970).

[20] van Neerven, J. The Asymptotic Behaviour of Semigroups of Linear Operators. Operator Theory Adv. Appl. Vol. 88, Basel: Birkhäuser (1996).

[21] Parthasarathy, K. R. Probability Measures on Metric Spaces. New York: Academic Press (1967).

[22] Parthasarathy, K. R. An Introduction to Quantum Stochastic Calculus. Basel: Birkhäuser (1992).

[23] Pedersen, G. K. $C^{*}$-Algebras and their Automorphism Groups. London: Academic Press (1979).

[24] SakaI, S. $C^{*}$-Algebras and $W^{*}$-Algebras. New York: Springer (1971).

[25] Schaefer, H. H. Topological Vector Spaces. 2nd ed. New York: Springer (1999).

[26] Schwartz, L. Radon Measures on Arbitrary Topological Vector Spaces and Cylindrical Measures. London: Oxford University Press (1973).

[27] Sewell, G. L. Quantum Physics and its Emergent Macrophysics. Princeton, N. J. : Princeton University Press (2002).

[28] Strocchi, F. An Introduction to the Mathematical Structure of Quantum Mechanics. Singapore: World Scientific (2005).

[29] Strocchi, F. Symmetry Breaking. Lecture Notes in Physics Vol. 643, Berlin: Springer (2005).

[30] Takesaki, M. Theory of Operator Algebras I. New York: Springer (1979).

[31] Upmeier, H. Jordan Algebras in Analysis, Operator Theory, and Quantum Mechanics. Providence, R I: American Math. Society (1987).

[32] Yosida, K. Functional Analysis. Berlin: Springer (1980). 


\section{Journal Articles}

[33] Aarnes, J. F. On the continuity of automorphic representations of groups. Commun. Math. Phys. 7, 332-336 (1968).

[34] Agarwal, G. S. Open quantum Markovian systems and the microreversibility. Z. Phys. A 258, 409-422 (1973).

[35] Akemann, C. A. The dual space of an operator algebra. Trans. Amer. Math. Soc. 126, 286-302 (1967).

[36] Alicki, R. On the detailed balance condition for non-Hamiltonian systems. Rep. Math. Phys. 10, 249-258 (1976).

[37] ARAKI, H. Hamiltonian formalism and the canonical commutation relations in quantum field theory. J. Math. Phys. 1, 492-504 (1960).

[38] Araki, H., Woods, E. J. Representations of the canonical commutation relations describing non-relativistic infinite free Bose gas. J. Math. Phys. 4, 637-662 (1963).

[39] Arendt, W., Chernoff, P. R., Kato, T. A generalization of dissipativity and positive semigroups. J. Operator Theory 8, 167-180 (1982).

[40] Arendt, W., Batty, C. J. K. Tauberian theorems for one-parameter semigroups. Trans. Amer. Math. Soc. 306, 837-852 (1988).

[41] Aspect, A., Dalibard, J., Roger, G. Experimental test of Bell's inequalities using time-varying analyzers. Phys. Rev. Lett. 49, 1804-1807 (1982).

[42] BaEz, J. Bell's inequality for C*-algebras. Lett. Math. Phys. 13, 135-136 (1987).

[43] Bahn, C., Ko, C. K., Park, Y. M. Dirichlet forms and symmetric Markovian semigroups on CCR algebras with respect to quasi-free states. J. Math. Phys. 44, 723-753 (2003).

[44] Benatti, F., Floreanini R. Open quantum dynamics: complete positivity and entanglement. Internat. J. Mod. Phys. B 19, 3063-3139 (2005).

[45] Benatti, F., Floreanini R. Tests of complete positivity in fiber optics. Open Syst. Inf. Dyn. 13, 229-238 (2006).

[46] Blanchard, Ph., Olkiewicz, R. Decoherence induced transition from quantum to classical dynamics. Rev. Math. Phys. 15, 217-243 (2003).

[47] Blanchard, Ph., Olkiewicz, R. Decoherence-induced continuous pointer states. Phys. Rev. Lett. 90, 010403 (2003).

[48] Blanchard, Ph., Olkiewicz, R. From quantum to quantum via decoherence. Phys. Lett. A 314, 29-36 (2003).

[49] Blanchard, Ph., Hellmich, M., Ługiewicz, P., Olkiewicz, R. Quantum dynamical semigroups for finite and infinite Bose systems. J. Math. Phys. 48, $012106(2007)$. 
[50] Blanchard, Ph., Hellmich, M., Ługiewicz, P., Olkiewicz, R. Continuity and generators of dynamical semigroups for infinite Bose systems. J. Funct. Anal. (in press).

[51] Bogachev, V. I., Röckner, M., Schmuland, B. Generalized Mehler semigroups and applications. Probab. Theory Relat. Fields 105, 193-225 (1996).

[52] Borchers, H. J. On the implementability of automorphism groups. Commun. Math. Phys. 14, 305-314 (1969).

[53] Bratteli, O., Robinson, D. W. Unbounded derivations of von Neumann algebras. Ann. Inst. H. Poincaré 25, 139-164 (1976).

[54] Cannon, J. T. Infinite volume limits of the canonical free Bose gas states on the Weyl algebra. Commun. Math. Phys. 29, 89-104 (1973).

[55] Carmichael, H. J., Walls, D. F. Detailed balance in open quantum Markoffian systems. Z. Phys. B 23, 299-306 (1976).

[56] Chог, M. D. Some assorted inequalities for positive linear maps on $C^{*}$-algebras. J. Operator Theory 4, 271-285 (1980).

[57] Christensen, E. Generators of semigroups of completely positive maps. Commun. Math. Phys. 62, 167-171 (1978).

[58] Cipriani, F. Dirichlet forms and Markovian semigroups on standard forms of von Neumann algebras. J. Funct. Anal. 147, 259-300 (1997).

[59] Clauser, J. F., Horne, M. A., Shimony, A., Holt, R. A. Proposed experiment to test local hidden variable theories. Phys. Rev. Lett. 23, 880-884 (1969).

[60] Davies, E. B. Markovian master equations. Commun. Math. Phys. 39, 91-110 (1974).

[61] Davies, E. B. Markovian master equations III. Ann. Inst. H. Poincaré 11, 265$273(1975)$.

[62] Davies, E. B. Markovian master equations II. Math. Ann. 219, 147-158 (1976).

[63] Davies, E. B. Irreversible dynamics of infinite Fermion systems. Commun. Math. Phys. 55, 231-258 (1977).

[64] Demoen, B., Vanheuverzwijn, P., Verbeure, A. Completely positive maps on the CCR-algebra. Lett. Math. Phys. 2, 161-166 (1977).

[65] Demoen, B., Vanheuverzwijn, P., Verbeure, A. Completely positive quasifree maps on the CCR algebra. Rep. Math. Phys 15, 27-39 (1979).

[66] Evans, D.E. Irreducible quantum dynamical semigroups. Commun. Math. Phys. 54, 293-297 (1977).

[67] Evans, D. E., Lewis, J. T. Some semigroups of completely positive maps on the CCR algebra. J. Funct. Anal. 26, 369-377 (1977). 
[68] Fagnola, F., Umanità, V. Generators of detailed balance quantum Markov semigroups. Inf. Dim. Anal. Quant. Probab. Relat. Topics 10, 335-363 (2007).

[69] Fredenhagen, K. On the modular structure of local algebras of observables. Commun. Math. Phys. 97, 79-89 (1985).

[70] Fonst, G. The Lévy-Hinčin representation of negative-definite functions. Z. Wahrscheinlichkeitstheorie verw. Gebiete 34, 313-318 (1976).

[71] Frigerio, A. Stationary states for quantum dynamical semigroups. Commun. Math. Phys. 63, 269-276 (1978).

[72] Frigerio, A., Gorini, V., Pulè, J. V. Open quasi-free systems. J. Statist. Phys. 22, 409-433 (1980).

[73] Frigerio, A., Gorini, V. Markov dilations and quantum detailed balance. Commun. Math. Phys. 93, 517-532 (1984).

[74] Fuhrman, M., Röckner, M. Generalized Mehler semigroups: the nonGaussian case. Potential Anal. 12, 1-47 (2000).

[75] Haag, R., Kadison, R. V., Kastler, D. Nets of $C^{*}$-algebras and classification of states. Commun. Math. Phys. 16, 81-104 (1970).

[76] HaAg, R., Kastler, D. An algebraic approach to quantum field theory. J. Math. Phys. 5, 848-861 (1964).

[77] Haag, R., Hugenholtz, N. M., Winnink, M. On the quilibrium states in quantum statistical mechanics. Commun. Math. Phys. 5, 215-236 (1967).

[78] Hellmich, M. Alicki's model of scattering-induced decoherence derived from Hamiltonian dynamics. J. Phys. A Math. Gen. 37, 8711-8719 (2004).

[79] Hellmich, M. Alicki's model of environmental decoherence by scattering: some mathematical results. Diploma Thesis, Univ. of Bielefeld (2004).

[80] Honegger, R. On the continuous extensions of states on the CCR algebra. Lett. Math. Phys. 42, 11-25 (1997).

[81] Hugenholtz, N. M. On the factor type of equilibrium states in quantum statistical mechanics. Commun. Math. Phys. 67, 189-193 (1967).

[82] Joos, E., ZEH, H. D. The emergence of classical properties through interaction with the environment. Z. Phys. B 52, 223-243 (1985).

[83] Kallman, R. A remark on a paper of J.F. Aarnes. Commun. Math. Phys. 14, 13-14 (1969).

[84] Kallman, R. Groups of inner automorphisms of von Neumann algebras. J. Funct. Anal. 7, 43-60 (1971).

[85] Kōmura, T. Semigroups of operators in locally convex spaces. J. Funct. Anal. 2, 258-296 (1968). 
[86] Kossakowski, A., Frigerio, A., Gorini, V., Verri, M. Quantum detailed balance and KMS condition. Commun. Math. Phys. 57, 97-110 (1977).

[87] Kümmerer, B., Nagel, R. Mean ergodic semigroups on $W^{*}$-algebras. Acta Sci. Math. (Szeged) 41, 151-159 (1979).

[88] Landau, L. J. On the violation of Bell's inequalities in quantum theory. Phys. Lett. A 120, 54-56 (1987).

[89] Landsman, N. P. Observation and superselection in quantum mechanics. Stud. Hist. Phil. Mod. Phys. 26, 45-73 (1995).

[90] Landsman, N. P. When champions meet: rethinking the Bohr-Einstein debate. Stud. Hist. Phil. Mod. Phys. 37, 212-242 (2006).

[91] Landsman, N.P. Between Classical and Quantum. In: J. Butterfield et.al. (eds.) Handbook of the Philosophy of Science: Philosophy of Physics. Amsterdam: North Holland (2007).

[92] Lewis, J. T., Pulè, J. V. The equilibrium states of the free Boson gas. Commun. Math. Phys. 36, 1-18 (1974).

[93] De Leeuw, K., Glicksberg, I. Applications of almost periodic compactifications. Acta Math. 105, 63-97 (1961).

[94] Ługiewicz, P., Olkiewicz, R. Classical properties of infinite quantum open systems. Commun. Math. Phys. 239, 241-259 (2003).

[95] Lyubich, Y. I., Quôc PhóNG VŨ Asymptotic stability of linear differential equations in Banach spaces. Studia Math. 88, 37-42 (1988).

[96] Majewski, W. A. On the relationship between the reversibility of dynamics and balance conditions. Ann. Inst. H. Poincaré 39, 45-54 (1983).

[97] Majewski, W. A. The detailed balance condition in quantum statistical mechanics. J. Math. Phys. 25, 614-616 (1984).

[98] Majewski, W. A. Return to equilibrium and stability of dynamics (semigroup dynamics case). J. Stat. Phys. 55, 417-433 (1989).

[99] Majewski, W.A., Streater, R. F. Detailed balance and quantum dynamical maps. J. Phys. A 31, 7981-7995 (1998).

[100] Majewski, W.A., Labuschagne, L. Quantum recurrences versus stability. Phys. Lett. A 322, 286-289 (2004).

[101] Manuceau, J. $C^{*}$-algèbre de relations de commutation. Ann. Inst. Henri Poincaré 8, 139-161 (1968).

[102] Manuceau, J., Verbeure, A. Quasi-free states of the C.C.R.-algebra and Bogoliubov transformations. Commun. Math. Phys. 9, 293-302 (1968).

[103] Manuceau, J., Sirugue, M., Testard, D., Verbeure, A. The smallest $C^{*}$ algebra for canonical commutation relations. Commun. Math. Phys. 32, 231-243 (1973). 
[104] Moffat, J. Continuity of automorphic representations. Proc. Camb. Phil. Soc. 74, 461-465 (1973).

[105] OlKIEWICZ, R. Environment-induced superselection rules in Markovian regime. Commun. Math. Phys. 208, 245-265 (1999).

[106] Olkiewicz, R. Structure of the algebra of effective observables in quantum mechanics. Ann. of Phys. 286, 10-22 (2000).

[107] Olkiewicz, R., ŻABA, M. Dynamics of microcavity polaritons in the Markovian limit. Phys. Lett. A 372, 3176-3138 (2008).

[108] PARK, Y.M. Construction of Dirichlet forms on standard forms of von Neumann algebras. Inf. Dim. Anal. Quant. Probab. Relat. Topics 3, 1-14 (2000).

[109] Parthasarathy, K.R., Rao, R. R., Varadhan, S. R. S. Probability distributions on locally compact abelian groups. Illinois J. Math. 7, 337-339 (1963).

[110] Pulè, J. V. The Bloch equations. Commun. Math. Phys. 38, 241-256 (1974).

[111] Raggio, G. A. A remark on Bell's inequality and decomposable normal states. Lett. Math. Phys. 15, 27-29 (1988).

[112] RÉdei, M., Summers, S. J. Quantum probability theory. Stud. Hist. Phil. Mod. Phys. 38, 390-417 (2007).

[113] Robinson, D. W. The ground state of the Bose gas. Commun. Math. Phys. 1, 159-174 (1965).

[114] Robinson, D. W. Strongly positive semigroups and faithful invariant states. Commun. Math. Phys. 85, 129-142 (1982).

[115] Robinson, D. W. Continuous semigroups on ordered Banach spaces. J. Funct. Anal. 51, 268-284 (1983).

[116] SaItô, K. A note on the continuity of automorphic representations of groups. Tôhoku Math. J. 28, 305-310 (1976).

[117] Schlosshauer, M. Decoherence, the measurement problem, and interpretations of quantum mechanics. Rev. Mod. Phys. 76, 1267-1305 (2004).

[118] Schmuland, B., Wei Sun On the equation $\mu_{t+s}=\mu_{s} * T_{s} \mu_{t}$. Stat. Probab. Lett. 52, 183-188 (2001).

[119] Segal, I. E. Postulates for general quantum mechanics. Ann. Math. 48, 930-948 (1947).

[120] Slawny, J. On factor representations and the $C^{*}$-algebra of canonical commutation relations. Commun. Math. Phys. 24, 151-170 (1972).

[121] Smolyanov, O. G., Fomin, S. V. Measures on linear topological spaces. Uspekhi Mat. Nauk. 31 (4), 1-53 (1976). Translation: Russian Math. Surveys 31 (4), 3-56 (1976). 
[122] Spohn, H. Kinetic equations from Hamiltonian dynamics: Markovian limits. Rev. Mod. Phys. 52, 569-615 (1980).

[123] Streater, R.F. Classical and quantum probability. J. Math. Phys. 41, 35563603 (2000).

[124] Takesaki, M. Conditional expectations in von Neumann algebras. J. Funct. Anal. 9, 306-321 (1972).

[125] Thomsen, K. Dispersiveness and positive contractive semigroups. J. Funct. Anal. 56, 348-359 (1984).

[126] Thomsen, K. E. Invariant states for positive operator semigroups. Studia Math. 81, 285-291 (1985).

[127] TомiYama, J. On the projection of norm one in $W^{*}$-algebras. Proc. Japan Acad. Sci. 33, 125-129 (1957).

[128] Tomiyama, J. On the projection of norm one in $W^{*}$-algebras II. Tôhoku Math. J. 10, 125-129 (1958).

[129] Tomiyama, J. On the projection of norm one in $W^{*}$-algebras III. Tôhoku Math. J. 11, 125-129 (1959).

[130] Van Daele, A. Quasi-equivalence of quasi-free states on the Weyl algebra. Commun. Math. Phys. 21, 171-191 (1971).

[131] Vanheuverzwijn, P. Generators for quasi-free completely positive semigroups. Ann. Inst. H. Poincaré 29, 123-138 (1978).

[132] Vanheuverzwijn, P. Erratum to [131]. Ann. Inst. H. Poincaré 30, 83 (1979).

[133] Verbeure, A. Detailed balance and equilibrium. Lecture Notes in Math. Vol. 1136, 504-515, Berlin: Springer (1985).

[134] ZeH, H. D. On the interpretation of measurement in quantum theory. Found. Phys. 1, 69-76 (1970).

[135] ZureK, W. H. Pointer basis of quantum apparatus: into what mixture does the wave packet collapse? Phys. Rev. D 24, 1516-1525 (1981).

[136] Zurek, W.H. Environment-induced superselection rules. Phys. Rev. D 26, 1862-1880 (1982).

[137] Zurek, W.H. Decoherence, einselection, and the quantum origins of the classical. Rev. Mod. Phys. 75, 715-775 (2003). 


\section{Summary}

Today quantum mechanics is considered as the most fundamental physical theory available. As such it is not limited to its traditional areas like atomic and elementary particle physics and quantum field theory, but its scope should also contain macroscopic systems, obeying the laws of classical physics. Here a fundamental problem arises: when the laws of quantum mechanics are directly applied to the objects of our everyday field of experience, contradictions arise. Probably the most famous example is the Schrödinger cat, which exists is a nonclassical state which is a superposition of the "dead" and "alive" state of the cat.

The program of environmental decoherence provides an answer to these problems. It contends that quantum mechanics is universally valid but that one has to take into account that macroscopic systems are usually strongly interacting with their environment. This interaction causes the time evolution of the system to become irreversible, and irreversibility is able to dynamically generate classical properties. Specifically, the interaction between system and environment leads to a strong entanglement between them which effectively renders phase relations between vectors from certain subspaces of the systems Hilbert space unobservable, thus limiting the superposition principle which is responsible for nonclassical behavior like in the Schrödinger cat example.

Despite the recent progress in our understanding of decoherence there is still a lot of discussion about the meaning and prospects of decoherence going on. In order to clarify the status of decoherence and to classify possible scenarios $\mathrm{Ph}$. Blanchard and R. Olkiewicz introduced a mathematically rigorous definition of decoherence in the algebraic framework of quantum physics. The algebraic framework is an alternative mathematical formulation of quantum physics which is more general than the traditional Hilbert space formulation; in particular, it permits the rigorous discussion of systems with infinitely many degrees of freedom occurring in quantum field theory or in the thermodynamical limit, as well as superselection rules and classical systems.

Up to now most studies on decoherence have been concentrating on systems describable by a finite-dimensional Hilbert space or standard quantum mechanics with finitely many degrees of freedom. The theory of 
irreversible dynamics and decoherence of systems with infinitely many degrees of freedom in contrast is far less advanced. It is the objective of this work to provide some general results about decoherence in systems with Markovian time evolution which are sufficiently general to cover also infinite systems. A second aspect is to construct irreversible dynamics on representations of the algebra of canonical commutation relations (CCR algebra) in a mathematically rigorous way and to study their properties.

Chapter 1 starts by introducing the algebraic framework of quantum physics, in particular, we emphasize that quantum theory may fruitfully be viewed as a generalization of classical Kolmogorovian probability theory. The necessary mathematical tools to describe the dynamics of open systems, in particular quantum dynamical semigroups, which are fundamental for decoherence are introduced as well. Next in Chapter 2 we develop mathematical tools which will allow us to prove that a given quantum dynamical semigroup displays the decoherence effect. Besides giving a new proof for a special case of a theorem of Ługiewicz and Olkiewicz we will also discuss the Jacobs-de Leeuw-Glicksberg splitting for quantum dynamical semigroups as well as a notion of detailed balance which will lead to a new sufficient condition for decoherence of a quantum dynamical semigroup. In Chapter 3 we introduce the notion of decoherence due to $\mathrm{Ph}$. Blanchard and R. Olkiewicz and discuss its consequences. Our results from Chapter 2 lead to a number of new conditions for a quantum dynamical semigroup to display decoherence. A class of quantum dynamical semigroups on von Neumann algebras corresponding to representations of the algebra of the canonical commutation relations, which describe (not necessarily finite) Bose systems is constructed in Chapter 4. Moreover, we achieve a complete description of these semigroups in terms of their generators and discuss the existence of faithful normal invariant states. We close with some open problems and proposals for future investigations in Chapter 5.

\section{Zusammenfassung in deutscher Sprache}

Die Quantenmechanik gilt heute als unsere grundlegendste physikalische Theorie. Als solche beschränkt sie sich nicht nur auf ihre ursprünglichen Anwendungsbereiche wie die Atomphysik, Elementarteilchenphysik und die Quantenfeldtheorie, sondern ihr Gegenstandsbereich sollte auch makroskopische Systeme einschließen, die den Gesetzen der klassischen Physik gehorchen. Hier stößt man jedoch auf ein fundamentales Problem: Wendet man die Gesetze der Quantenmechanik direkt auf die Objekte un- 
serer Alltagswelt an, so gelangt man zu Widersprüchen. Der wahrscheinlich berühmteste ist die Schrödinger-Katze, die sich in einem nichtklassischen Zustand befindet, der eine kohärente Überlagerung des Zustandes „tot“ und "lebendig" der Katze darstellt.

Die Theorie der Dekohärenz bietet eine Lösung dieser Probleme. Sie geht davon aus, daß die Quantenmechanik universell gültig ist, man jedoch zu berücksichtigen hat, daß makroskopische Systeme gewöhnlich stark mit ihrer Umgebung in Wechselwirkung stehen. Durch diese Wechselwirkung wird die Zeitentwicklung des betrachteten Systems irreversibel, und diese Irreversibilität ist in der Lage, klassische Effekte dynamisch zu erzeugen. Genauer gesprochen bewirkt die Wechselwirkung eine starke Verschränkung zwischen System und Umgebung, welche Phasenfaktoren zwischen Vektoren bestimmter Unterräume des Hilbertraumes des Systems unbeobachtbar macht und somit das Superpositionsprinzip einschränkt, welches nichtklassische Zustände wie im Beispiel der Schrödinger-Katze erlaubt.

Trotz des großen Fortschritts im Verständnis der Dekohärenz, der in den letzten Jahren erreicht wurde, ist die Frage nach ihren Grundlagen und Möglichkeiten heute immer noch Gegenstand aktiver Diskussionen. Um den Status der Dekohärenz zu klären und ihre verschiedenen möglichen Szenarien zu klassifizieren, wurde von Ph. Blanchard und R. Olkiewicz ein mathematisch rigoroser Begriff der Dekohärenz im Rahmen der algebraischen Quantenphysik vorgeschlagen. Die algebraische Quantenphysik ist eine alternative und allgemeinere mathematische Formulierung der Quantenmechanik als die traditionelle Hilbertraumformulierung. Insbesondere ermöglicht sie eine mathematisch rigorose Behandlung von Systemen mit unendlich vielen Freiheitsgraden, welche in der Quantenfeldtheorie oder im thermodynamischen Limes vorkommen; außerdem lassen sich besonders einfach Superauswahlregeln sowie klassische Systeme beschreiben.

Bisher konzentrierten sich die meisten Arbeiten zur Dekohärenz auf Systeme mit endlichdimensionalem Hilbertraum oder auf Systeme mit endlich vielen Freiheitsgraden. Die Theorie der Dekohärenz und irreversibler Zeitentwicklungen für Systeme mit unendlich vielen Freiheitsgraden ist weit weniger fortgeschritten. Es ist das Ziel der vorliegenden Arbeit, einige allgemeine Resultate über Dekohärenz in Systemen mit Markoffscher Zeitentwicklung beizusteuern, die hinreichend allgemein sind um auch für unendliche Systeme ihre Gültigkeit zu behalten. Ein zweiter Aspekt ist die mathematisch rigorose Konstruktion von irreversiblen Zeitentwicklungen auf Darstellungen der Algebra der kanonischen Vertauschungsrelationen (CCR-Algebra).

Das Kapitel 1 beginnt mit einer Einführung in die algebraische Formulie- 
rung der Quantenphysik, insbesondere wird betont, daß der Formalismus der Quantenphysik sich als Verallgemeinerung der klassischen Kolmogoroffschen Wahrscheinlichkeitstheorie auffassen läßt. Die notwendigen mathematischen Werkzeuge für eine Beschreibung irreversibler Zeitentwicklungen im algebraischen Rahmen werden ebenfalls zur Verfügung gestellt. In Kapitel 2 werden einige mathematische Resultate entwickelt, die es zu beweisen erlauben, daß eine dynamische Halbgruppe Dekohärenz zeigt. Neben einem neuen Beweis eines Theorems von Ługiewicz und Olkiewicz wird die Jacobs-de Leeuw-Glicksberg-Zerlegung für dynamische Halbgruppen betrachtet sowie eine Detailed Balance-Bedingung, die im Hinblick auf Anwendungen zur Dekohärenz untersucht wird. Das Kapitel 3 führt den Begriff der Dekohärenz nach Ph. Blanchard und R. Olkiewicz ein und diskutiert einige seiner Konsequenzen. Die Resultate aus Kapitel 2 erlauben die Formulierung einiger neuer Kriterien für Dekohärenz einer dynamischen Halbgruppe. Eine Klasse von dynamischen Halbgruppen auf Darstellungen der CCR-Algebra, die (nicht notwendigerweise endliche) bosonische Systeme beschrieben, wird in Kapitel 4 konstruiert. Eine vollständige Beschreibung dieser Halbgruppen durch ihre Generatoren wird gegeben, außerdem wird die Existenz eines treuen invarianten Zustandes diskutiert. Das Kapitel 5 schließt mit einigen Bemerkungen zu offenen Problemen und Vorschlägen für zukünftige Untersuchungen. 
Gedruckt auf alterungsbeständigem Papier nach DIN-ISO 9706 